NIST Special Publication 1002

\title{
Ultra-High Molecular Weight Polyethylene
}

\section{Wear Particles Effects on Bioactivity}

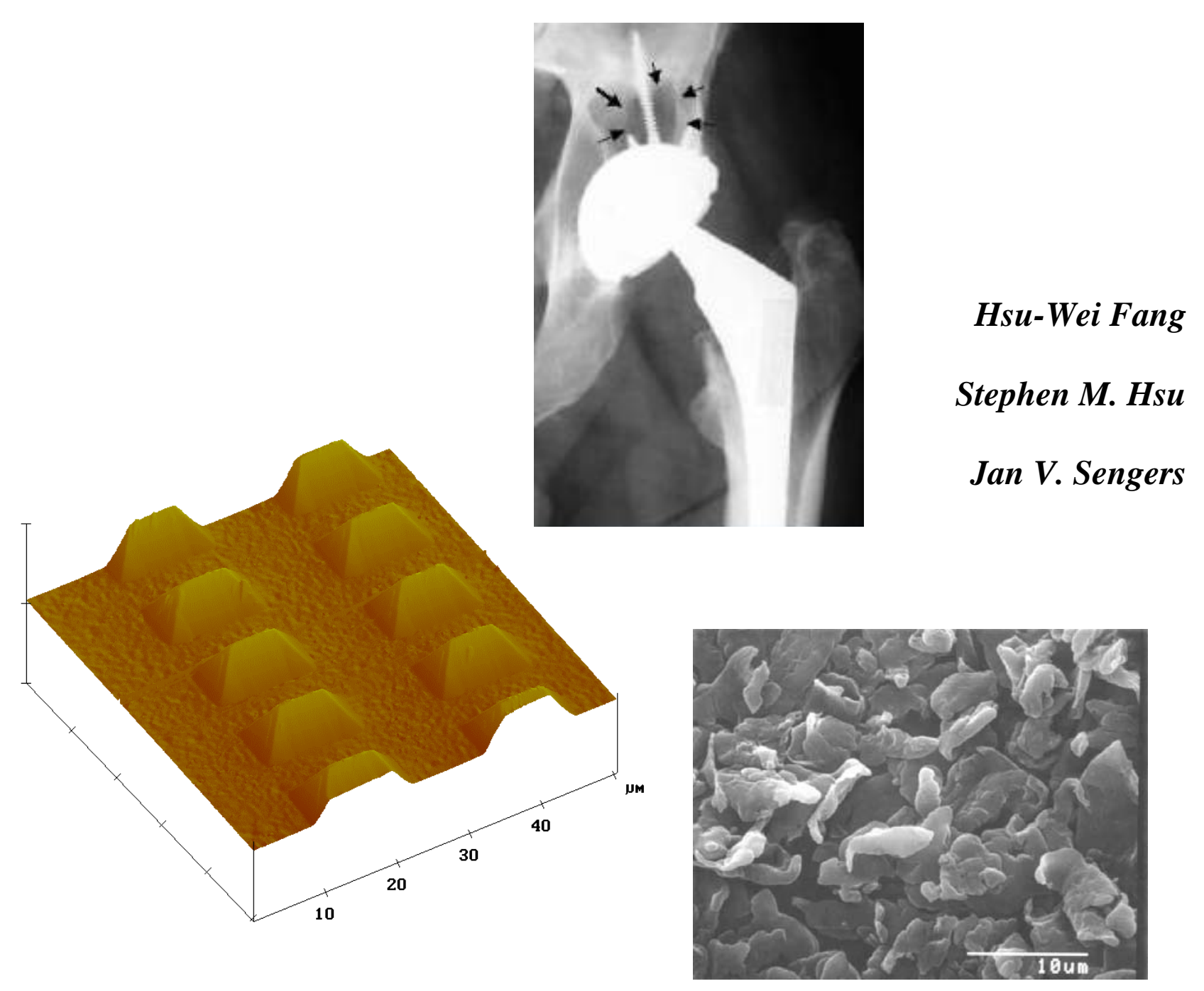




\title{
Ultra-High Molecular Weight Polyethylene Wear Particle Effects on Bioactivity
}

\author{
Hsu-Wei Fang ${ }^{1,3}$ \\ Stephen M. Hsu ${ }^{1}$ \\ Jan V. Sengers ${ }^{2,3,4}$ \\ ${ }^{1}$ Material Science and Engineering Laboratory \\ National Institute of Standards and Technology \\ Gaithersburg, MD 20899-8520 \\ ${ }^{2}$ Chemical Science and Technology Laboratory \\ National Institute of Standards and Technology \\ Gaithersburg, MD 20899-8380 \\ ${ }^{3}$ Department of Chemical Engineering \\ University of Maryland \\ College Park, MD 20742 \\ ${ }^{4}$ Institute for Physical Science and Technology \\ University of Maryland \\ College Park, MD 20742
}

September 2003

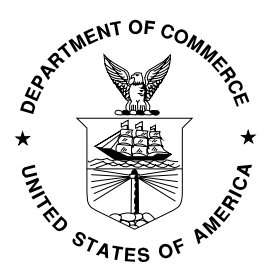

U.S. Department of Commerce Donald L. Evans, Secretary

Technology Administration

Phillip J. Bond, Under Secretary for Technology

National Institute of Standards and Technology Arden L. Bement, Jr., Director 
Certain commercial entities, equipment, or materials may be identified in this document in order to describe an experimental procedure or concept adequately. Such identification is not intended to imply recommendation or endorsement by the National Institute of Standards and Technology, nor is it intended to imply that the entities, materials, or equipment are necessarily the best available for the purpose.

National Institute of Standards and Technology Special Publication 1002 Natl. Inst. Stand. Technol. Spec. Publ. 1002, 277 pages (September 2003) CODEN: NSPUE2

For sale by the Superintendent of Documents, U.S. Government Printing Office Internet: bookstore.gpo.gov — Phone: (202) 512-1800 — Fax: (202) 512-2250 


\section{PREFACE}

This report is the result of a cooperative research project between the Chemical Engineering Department of University of Maryland and the National Institute of Standards and Technology. Within NIST, Dr. John Tesk of the Polymers Division has provided continued support and guidance.

The project was initiated as a direct result of the NIST Orthopaedic Wear Consortium established by John Tesk and Stephen Hsu. One of the ongoing concerns of the biomedical community is how to test the bioactivity of wear particles generated by the artificial joints. Mr. Hsu-Wei Fang at University of Maryland chose this topic as his $\mathrm{Ph} . \mathrm{D}$. thesis and this report is a summary of his research in the past five years.

Prof. Jan Sengers lent his support to this project by serving as co-advisor to Mr. Fang and helped guide the thesis research to a successful conclusion. In this report, you will find how wear particles induce bone loosening, how particles can be

generated, the particle formation mechanisms, and theoretical models describing how to control the size and shape of particles using microfabricated surface textures. Bioactivity tests on particles were performed by the Federal Food and Drug Administration and the Wayne State University through collaborations. We sincerely thank them for their technical assistances.

It is always a pleasure to work with someone like Dr. Hsu-Wei Fang who has persevered through the past five years to reach a successful goal. Cooperative research such as this furthers one's educational goals while serving the programmatic goals of NIST. This is a model that merits encouragement.

Stephen M. Hsu

Leader, Nanotribology Group

Ceramics Division

Materials Science and Engineering Laboratory 


\begin{abstract}
Ultra-high molecular weight polyethylene (UHMWPE) wear particles have been recognized as one of the major causes of aseptic loosening in total joint replacements. Macrophage phagocytosis of wear particles induces human biological/physiological responses which eventually lead to bone resorption and osteolysis. However, the dependence of these reactions on the size and shape of the particles has not been elucidated and is not understood. The goal of this study is to develop a technique for producing UHMWPE particles with specified sizes and shapes so as to be able to study the effects of different UHMWPE particles on bioactivity. We applied surface texturing techniques to generate UHMWPE particles and air pouch animal tests to study the biological responses induced by UHMWPE particles.

The study describes a procedure for generating narrowly distributed UHMWPE particles with controlled size and shape through surface texturing by microfabrication. The textured surface is used to rub against the polymer pins to produce wear particles in water. The surface texture produces narrowly distributed elongated particles or equiaxed particles by design. Experimental results show that the cutting-edge length of the surface features is proportional to the particle length. A larger penetration depth, larger normal load, and smaller sliding speed lead to a smaller aspect ratio of the particle. With this technique, we have been able to generate UHMWPE wear particles with different size and shape within phagocytosable and non-phagocytosbale ranges for biological response studies.
\end{abstract}


A mathematical model to predict the dimensions of generated UHMWPE particles has been developed. A correlation model based on the empirical results is also presented. The models can be used to design the dimensions of the surface textures and the operating conditions of the wear tests for generation of the particle population with specified size and shape.

Murine air pouch animal tests have been used to test the biological responses induced by UHMWPE particles. The results indicated elongated particles induced stronger immunological responses than round-like particles. UHMWPE particles that can be phagocytosed by the macrophage cells stimulated a higher bioactivity than the non-phagocytosable particles. This paves the way for future material development and textural designs that can minimize the most toxic wear particle generation, hence prolong the life of orthopaedic joint replacements. 


\section{TABLE OF CONTENTS}

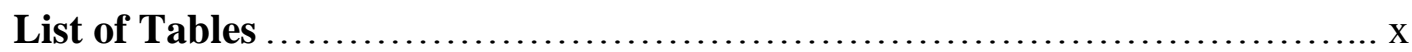

List of Figures ........................................................ xi

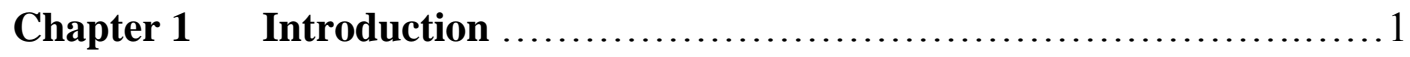

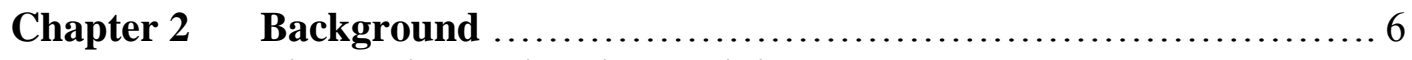

2.1 The need to replace human joints ........................... 6

2.2 Total joint replacement ..................................... 7

2.2.1 Function and components .......................... 7

2.2.2 Material properties of UHMWPE and their implications ....9

2.3 Failure of total joint replacement ............................. 12

2.3.1 Wear and wear particles of UHMWPE components ....... 12

2.3.2 Wear particles induced osteolysis ....................... 16

2.4 Wear studies of UHMWPE material ........................... 18

2.5 Evaluation of bioactivity of UHMWPE particles ............... 22

2.5.1 Wear particle induced reactions \& mechanisms ........... 22

2.5.2 In-vitro cell culture model study ........................ 24

2.5.3 In-vivo animal tests .................................. 24

Chapter 3 Problem definition ....................................... 26

3.1 The need to prolong the life of joint-replacement components .....26 26

3.2 Potential solutions if particle induced bioactivity is understood ....22

3.3 The cause and effect of particle sizes and shapes .................28

3.4 Critical issues ..................................................29

3.4.1 Availability of narrowly distributed particles ..............29

3.4.2 Characterization of particles ........................... 31

3.4.3 Bioactivity tests of UHMWPE particles .................... 31

$3.5 \quad$ Approaches ............................................ 32

3.5.1 Generation of particles by surface texturing ...............32

3.5.2 Modeling of particle-generation process ................ 32

3.5.3 Characterization of particles .......................... 33

3.5.4 Bioactivity tests of UHMWPE particles ................ 33

Chapter 4 Procedures for generation and collection of particles ........... 34

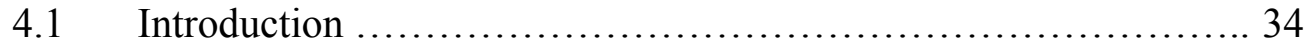

4.2 Generation of UHMWPE particles ........................... 35

4.2.1 Literature review of UHMWPE particle generation .........35

4.2.2 Concepts of surface texturing ........................... 36

4.2.3 Preparation of materials ............................. 37 
4.2.4 Linear reciprocating wear process......................... 39

4.3 Collection of UHMWPE particles ........................... 41

4.3.1 Particle-collection procedure .......................... 41

4.3.2 Effect of $\mathrm{pH}$ value on particle recovery ..................44

$4.4 \quad$ Particle characterization ..................................... 47

4.4.1 Chemical composition of particles ......................47

4.4.2 Image analysis of particles ...........................47

Chapter 5 Particle generation with scratched steel surfaces ............. 49

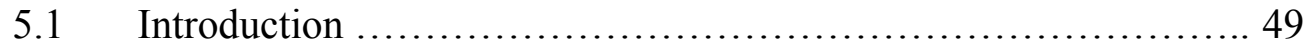

5.2 Particle generation with abrasive-paper made surfaces ............49

5.2.1 Preparation of steel surfaces with $\mathrm{SiC}$ abrasive papers ..... 49

5.2 .2 Results ..............................................50

5.3 Effect of surface-texture pattern on particle shape ..................58

5.4 Effect of surface-texture roughness on particle size ...............60

5.5 Particle generation with diamond-tip scratched surfaces ...........662

5.5.1 Preparation of diamond-tip scratched stainless steel surfaces

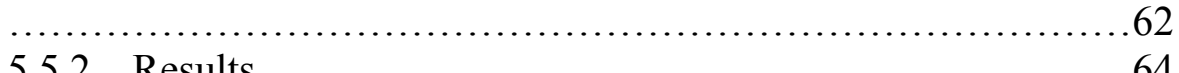

5.6 Effect of surface uniformity on particle distribution ............. 68

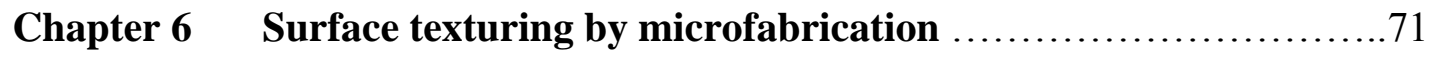

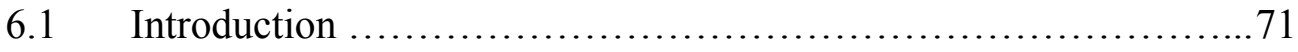

6.2 Surface texture with wedge features .......................... 71

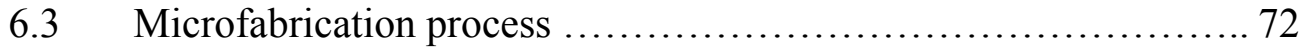

6.3.1 Substrate material ................................... 72

6.3.2 Masking material .................................... 74

6.3.3 Pattern design and transfer .......................... 75

6.3.4 Fabrication of surface features ....................... 76

6.3.5 Wear-resistant coating ............................... 79

6.4 Measurement of the surface texture .............................. 80

Chapter 7 Surface-texture design and generation of narrowly distributed particles .............................................. 82

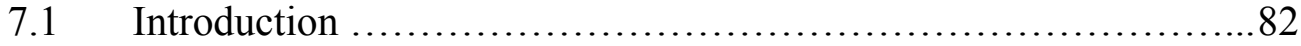

7.2 Surface-texture designs ................................. 82

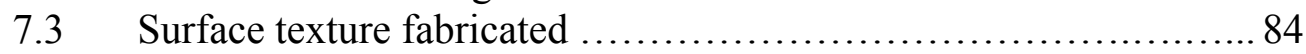

$7.4 \quad$ Results.................................................. 86

7.4.1 Effect of the cutting-edge length ...................... 86

7.4.2 Effect of the cutting-edge height ....................... 91

7.4.3 Effects of the cutting edge pattern .........................93

7.4.4 Effect of pitch distance ................................. 100

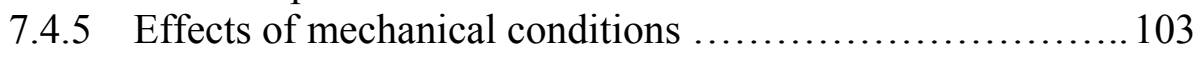

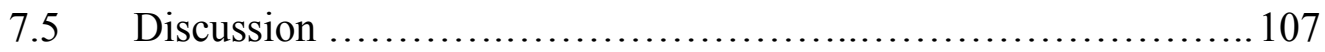


7.5.1 Effect of surface-texture uniformity on particle distribution. 107

7.5.2 Significance for bioactivity tests .............................109

7.6 Conclusions ............................................... 111

Chapter 8 Mechanism of UHMWPE particle generation …............... 112

$8.1 \quad$ Introduction .................................................. 112

8.2 Penetration of the cutting edge ............................... 113

8.3 Sliding of the cutting edge....................................... 118

8.3.1 Simple model experiments with constant load .............. 118

8.3.2 Lateral displacement of the tip ............................120

8.3 .3 Reacting forces ........................................ 123

8.3.4 Effect of normal load and cutting-edge angle ...............125

8.4 Shear induced molecular orientation of UHMWPE ............... 128

8.5 Strain-hardening effect .................................... 131

8.5.1 Constant depth sliding model experiments .................. 133

8.6 Observations from the particle-generation results ................. 141

8.7 Conclusions ........................................................ 144

Chapter 9 Modeling of particle generation .............................. 145

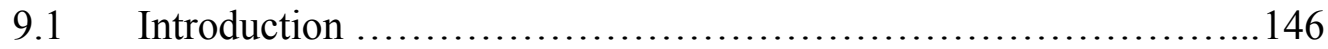

$9.2 \quad$ Model parameters ................................................. 147

9.3 Contact mechanics modeling .................................. 148

9.3.1 Penetration of the cutting edge ......................... 148

9.3.2 Sliding process of the cutting edge ......................... 153

9.3.3 Validation of the model ..................................... 159

$9.4 \quad$ Correlation model ............................................ 164

9.4.1 Effect of cutting-edge length .............................. 164

9.4.2 Effect of penetration depth .............................166

9.4.3 Effect of load .......................................... 168

9.4.4 Effect of sliding speed .................................. 169

9.4.5 Comparison with experimental results ..................... 170

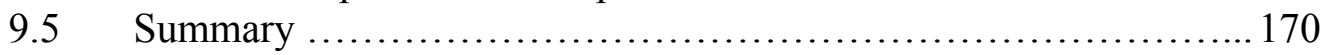

\section{Chapter 10 Particle characterization by analyzing the phagocytosis of} particles ...................................................... 172

10.1 Introduction ................................................... 172

10.2 Basic particle parameters ..................................... 173

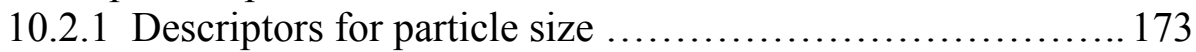

10.2.2 Descriptors for particle shape ............................ 175

10.3 Osteolysis induced by UHMWPE particles ...................... 178

10.4 Phagocytosis of particles ....................................... 180

10.4.1 Criteria for particle internalization by macrophage cells......182

10.4.2 Concept of phagocytic capacity ......................... 183 
10.4.3 Critical threshold for phagocytosis of particles with different shapes................................................. 185

10.5 Validation against literature results ............................. 187

10.6 Effect of excess particles ...................................... 193

10.7 Conclusions ....................................................... 195

Chapter 11 Bioactivity tests of UHMWPE particles ........................196

11.1 Introduction .................................................. 196

11.2 Murine air pouch animal test .................................. 197

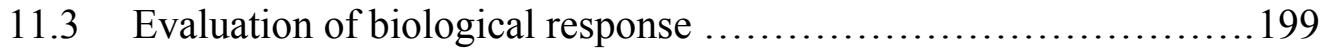

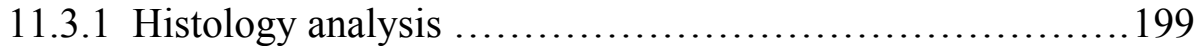

11.3.2 Measurement of cytokines ..............................202

11.4 Effect of particle shape ..................................... 203

11.5 Effect of particle size ....................................... 207

11.6 Effect of particle dosage ..................................... 208

11.7 Combined effects from size, shape and dosage of particles ......... 209

11.8 Conclusion ..................................................... 215

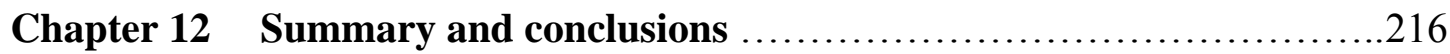

12.1 Design of surface textures for particle generation ..................2.216

12.2 Modeling of UHMWPE particle generation ......................2. 217

12.3 Bioactivity of UHMWPE particles ............................... 219

Chapter 13 Future work and recommendations ...........................221

13.1 Improvements of current techniques of particle generation .........221

13.2 Mechanism study for osteolysis induced by particles .............. 222

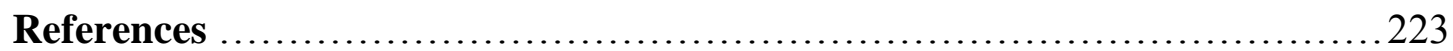

Appendix A Publications and awards .................................... 241

Appendix B Microfabrication of steel surface by electrochemical etching .....245

Appendix C Surface texture and wear data …................................. 249

Appendix D Modeling of particle generation by correlation of model Experimental data ..............................................251

Appendix E Matlab program for contact mechanics model of particle

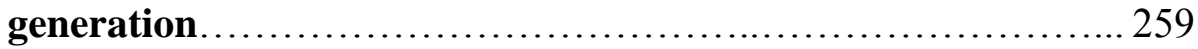




\section{LIST OF TABLES}

Table 2.1 Typical physical properties of HDPE and UHMWPE [21]...... 9

Table 2.2 Summary of retrieval UHMWPE wear particle studies in the literature................................................ 14

Table 2.3 Definition of biological terms related to osteolysis............. 17

Table 2.4 Summary of bioactivity study of UHMWPE particles from literature........................................... 23

Table 5.1 Results of particle generation with abrasive paper made surfaces.................................................... 53

Table 5.2 Effect of roughness of steel surfaces on particle dimensions...... 60

Table 5.3 Results of particle generation with diamond-tip scratched stainless steel surfaces...................................... 64

Table 7.1 Value of $\left(D_{\mathrm{s}} / H_{\mathrm{c}}\right)$ for effective and non-effective micro-cutting processes......................................................

Table 8.1 Comparison of penetration depth for the cutting edges with different angles under various normal loads.................. 117

Table 10.1 Summary of in-vitro cell culture results from Catelas et al. [79].. 188

Table 10.2 Summary of in-vitro cell culture results from Green et al. [81]... 190 


\section{LIST OF FIGURES}

Figure 2.1 Components of total hip and knee joint replacements........... 8

Figure 2.2 Schematic diagram of the chain orientation of UHMWPE....... 10

Figure 2.3 Examples of worn UHMWPE components for total hip and knee replacements...................................... 13

Figure 2.4 Examples of UHMWPE particles retrieved from (a) total hip replacement, and (b) total hip replacement $[41] \ldots \ldots \ldots \ldots \ldots \ldots . . . .15$

Figure 2.5 Osteolysis taking place around the total hip replacements which is detected by $\mathrm{X}$-ray diagnosis...............................

Figure 2.6 An example of forces acting on the hip-joint implant during gait [67]....

Figure 2.7 Trajectories of contact points on the articular surface of hipjoint implant [74] and hip-joint kinematic data during gait [73]..

Figure 3.1 Comparison of polyethylene powders and particles............ 30

Figure 4.1 Weight change of pre-soaked UHMWPE pins................ 38

Figure 4.2 Schematic representation of linear reciprocating wear tester..... 40

Figure 4.3 Particle-collection procedure............................. 43

Figure 4.4 Effect of $\mathrm{pH}$ value of water on removing particles on the glass slide

Figure 4.5 Effect of $\mathrm{pH}$ value of water on particle-recovery rate............ 46

Figure 4.6 IR absorbance spectra of UHMWPE bulk material and of collected particles........................................ 48

Figure 5.1 Procedure of surface texturing with $\mathrm{SiC}$ abrasive papers......... 51

Figure 5.2 (a) Unidirectional and (b) cross-hatched surface textures made on 316 steel plate........................................ 52 
Figure 5.3 Particles generated with 120 grit abrasive paper made unidirectional stainless steel surface. (a) SEM micrograph, (b) Distribution of particle length, (c) Distribution of particle aspect ratio

Figure 5.4 Particles generated with 320 grit abrasive paper made unidirectional stainless steel surface. (a) SEM micrograph, (b) Distribution of particle length, (c) Distribution of particle aspect ratio.

Figure 5.5 Particles generated with 120 grit abrasive paper made crosshatched stainless steel surface. (a) SEM micrograph, (b) Distribution of particle length, (c) Distribution of particle aspect ratio

Figure 5.6 Particles generated with 320 grit abrasive paper made crosshatched stainless steel surface. (a) SEM micrograph, (b) Distribution of particle length, (c) Distribution of particle aspect ratio.

Figure 5.7 Comparison of particle aspect-ratio distribution from abrasive paper made stainless steel surfaces with different surface-texture styles.

Figure 5.8 Comparison of particle-length distribution from stainless steel surfaces made by different grades of abrasive papers.............. 6

Figure 5.9 Procedure of surface texturing by diamond scratching........... 63

Figure 5.10 SEM micrographs diamond-tip scratched surfaces: (a) with linear scratches, (b) with a $60 \mu \mathrm{m}$ scratched length

Figure 5.11 Particles generated with the diamond-tip scratched stainless steel surface (linear type) (a) SEM micrograph, (b) Distribution of particle length, (c) Distribution of particle aspect ratio....

Figure 5.12 Particles generation with the diamond-tip scratched stainless steel surface (scratched distance $=60 \mu \mathrm{m})($ a) SEM micrograph, (b) Distribution of particle length, (c) Distribution of particle aspect ratio

Figure 5.13 Examples of surface profiles for (a) $\mathrm{SiC}$ abrasive-paper made steel surface, and (b) Diamond-tip scratched stainless steel surface 
Figure 5.14 Comparison of standard deviation and mean particle length for different particle-size populations made with surfaces made with SiC abrasive papers and with diamond-tip scratching process.................................................

Figure 6.1 Schematic of microfabrication process to generate wedge features on silicon.

Figure 6.2 Schematic representation of the wedge geometry controlled by anisotropic and isotropic etching processes................... 78

Figure 6.3 SEM micrographs of the wedge surface-features made by the microfabrication process...................................

Figure 6.4 Measurement of the three-dimensional geometry of the wedge surface textures by atomic force microscopy (AFM)..............

Figure 7.1 Schematic of the surface texture with wedge features.............

Figure 7.2 A schematic representation of dimensions of the surface textures containing wedge-shaped cutting edges........................

Figure 7.3 Micrographs of the surface texture A-1 $\left(L_{\mathrm{c}}=55 \mu \mathrm{m}, H_{\mathrm{c}}=4.2\right.$ $\mu \mathrm{m}, D_{\mathrm{s}}=200 \mu \mathrm{m}$ ) and generated UHMWPE particles (contact pressure $=3 \mathrm{MPa}$, average sliding speed $=57.2 \mathrm{~mm} / \mathrm{s}) \ldots \ldots \ldots \ldots$

Figure 7.4 Micrographs of the surface texture A-2 $\left(L_{\mathrm{c}}=15.0 \mu \mathrm{m}, H_{\mathrm{c}}=3.4\right.$ $\mu \mathrm{m}, D_{\mathrm{s}}=200 \mu \mathrm{m}$ ) and generated UHMWPE particles (contact pressure $=3 \mathrm{MPa}$, average sliding speed $=57.2 \mathrm{~mm} / \mathrm{s}) \ldots \ldots \ldots \ldots$

Figure 7.5 Micrographs of the surface texture A-3 $\left(L_{\mathrm{c}}=7 \mu \mathrm{m}, H_{\mathrm{c}}=3.0 \mu \mathrm{m}\right.$, $D_{\mathrm{s}}=200 \mu \mathrm{m}$ ) and generated UHMWPE particles (contact pressure $=3 \mathrm{MPa}$, average sliding speed $=57.2 \mathrm{~mm} / \mathrm{s}) \ldots \ldots \ldots \ldots$

Figure 7.6 Comparison of UHMWPE particles generated from surface texture A-2 with different cutting-edge heights (contact pressure $=3 \mathrm{MPa}$, average sliding speed $=57.2 \mathrm{~mm} / \mathrm{s}$ )

Figure 7.7 Micrographs of the surface texture A-4 $\left(L_{\mathrm{c}}=6.8 \mu \mathrm{m}, H_{\mathrm{c}}=2.3\right.$ $\mu \mathrm{m}, D_{\mathrm{s}}=10 \mu \mathrm{m}$ ) and generated UHMWPE particles (contact pressure $=3 \mathrm{MPa}$, average sliding speed $=57.2 \mathrm{~mm} / \mathrm{s}) \ldots \ldots \ldots \ldots$

Figure 7.8 Micrographs of the surface texture A-5 $\left(L_{\mathrm{c}} 3.2 \mu \mathrm{m}, H_{\mathrm{c}}=0.8 \mu \mathrm{m}\right.$, 
$D_{\mathrm{s}}=4 \mu \mathrm{m}$ ) and generated UHMWPE particles (contact pressure

$=3 \mathrm{MPa}$, average sliding speed $=57.2 \mathrm{~mm} / \mathrm{s}$ )....

Figure 7.9 Micrographs of the surface texture B-1 $\left(L_{\mathrm{c}}=6.2 \mu \mathrm{m}, H_{\mathrm{c}}=1.3\right.$ $\mu \mathrm{m}, D_{\mathrm{s}}=10 \mu \mathrm{m}$ ) and generated UHMWPE particles (contact pressure $=3 \mathrm{MPa}$, average sliding speed $=57.2 \mathrm{~mm} / \mathrm{s}) \ldots \ldots \ldots \ldots$

Figure 7.10 Micrographs of the surface texture B-4 $\left(L_{\mathrm{c}}=3.8 \mu \mathrm{m}, H_{\mathrm{c}}=1.2\right.$ $\mu \mathrm{m}, D_{\mathrm{s}}=10 \mu \mathrm{m}$ ) and generated UHMWPE particles (contact pressure $=3 \mathrm{MPa}$, average sliding speed $=57.2 \mathrm{~mm} / \mathrm{s}) \ldots \ldots \ldots \ldots$

Figure 7.11 Micrographs of the surface texture B-2 $\left(L_{\mathrm{c}}=3.5 \mu \mathrm{m}, H_{\mathrm{c}}=1.4\right.$ $\mu \mathrm{m}, D_{\mathrm{s}}=20 \mu \mathrm{m}$ ) and generated UHMWPE particles (contact pressure $=3 \mathrm{MPa}$, average sliding speed $=57.2 \mathrm{~mm} / \mathrm{s}) \ldots \ldots \ldots \ldots$

Figure 7.12 Micrographs of the surface texture B-3 $\left(L_{\mathrm{c}}=3.6 \mu \mathrm{m}, H_{\mathrm{c}}=0.6\right.$ $\mu \mathrm{m}, D_{\mathrm{s}}=10 \mu \mathrm{m}$ ) and generated UHMWPE particles (contact pressure $=3 \mathrm{MPa}$, average sliding speed $=57.2 \mathrm{~mm} / \mathrm{s}) \ldots \ldots \ldots \ldots$

Figure 7.13 Comparison of UHMWPE particles generated under different normal loads (by using the surface texture A-3 with cutting edge length $=7 \mu \mathrm{m}$, average sliding speed $=57.2 \mathrm{~mm} / \mathrm{s}$ ).

Figure 7.14 Comparison of micrographs of UHMWPE particles generated by the surface texture A-1 under $3 \mathrm{MPa}$ contact pressure and sliding speeds of $27.6 \mathrm{~mm} / \mathrm{s}$ and $57.2 \mathrm{~mm} / \mathrm{s}$ respectively.

Figure 7.15 Comparison of particle length distribution between particles generated from a $\mathrm{SiC}$ abrasive paper made surface texture and a microfabricated surface texture.

Figure 7.16 Comparison of two particle populations with distinct particlelength distribution...

Figure 8.1 Load-displacement curve of nano-indentation test on UHMWPE with constant strain rate of $0.1 \mathrm{~s}^{-1}$....

Figure 8.2 Elastic modulus data for UHMWPE under different penetration depths from a nano-indentation test ( strain rate $\left.=0.1 \mathrm{~s}^{-1}\right) \ldots \ldots \ldots$

Figure 8.3 Schematic representation of scaled-up single cutting-edge sliding test with fixed normal-load condition 
Figure 8.4 Video frames of a cutting edge lift-up process.....................

Figure 8.5 Schematic representation of the cutting-edge sliding process over UHMWPE material with fixed normal load.

Figure 8.6 Force curve of the sliding process by a $90^{\circ}$ wedge-shaped cutting edge under a normal load of $66.75 \mathrm{~N}$.

Figure 8.7 Force curve of the sliding process by a $60^{\circ}$ wedge-shaped cutting edge under a normal load of $66.75 \mathrm{~N}$

Figure 8.8 Sliding distance between two peaks under different normal loads with two cutting-edge angles.

Figure 8.9 Schematic representation of the resolved component forces of the material resistant force in the vertical and horizontal direction during the sliding process...............................

Figure 8.10 Soft $\mathrm{x}$-ray absorption spectra showing the molecular orientation of surface layer after 5000 unidirectional sliding cycles. E= electric field [27].

Figure 8.11 Soft $x$-ray absorption spectra showing the degree of molecular alignment of UHMWPE surface layer after 5000 unidirectional sliding cycles [26].

Figure 8.12 True stress-strain curves for UHMWPE (GUR 4150) [104].......

Figure 8.13 True stress-strain curves for UHMWPE 4150 up to 0.12 strain in uniaxial tension and compression and elastic-plastic model to describe the behavior [104]

Figure 8.14 A schematic representation of the displacement controlled single cutting edge sliding test.

Figure 8.15 Horizontal-force curve of the cutting-edge sliding process (cutting-edge length $=2 \mathrm{~mm}$, penetration depth $=400 \mu \mathrm{m}$, sliding speed $=0.5 / \mathrm{s})$

Figure 8.16 Video frames of the cutting edge sliding process (cutting edge length $=2 \mathrm{~mm}$, penetration depth $=400 \mu \mathrm{m}$, sliding speed $=$ $0.5 / \mathrm{s})$

Figure 8.17 Horizontal force versus sliding distance for different penetration 
depths in a displacement-controlled cutting-edge sliding test.............................................................

Figure 8.18 Horizontal force as a function of the sliding distance for different cutting-edge angles in a displacement-controlled cutting-edge sliding test.

Figure 8.19 SEM micrographs of surface cutting-edge feature, UHMWPE worn surface and generated UHMWPE particles

Figure 8.20 UHMWPE wear particles with irregular edges and straight line indent marks made by cutting edges....

Figure 9.1 Illustrations of significant variables for the particle-generation process.

Figure 9.2 Schematic of the penetration process of the cutting edges

Figure 9.3 Comparison of the empirical data of wedge indentation in UHMWPE with elastic-plastic calculation results

Figure 9.4 Schematic representation of the sliding process of the cutting edge.

Figure 9.5 A flow chart of calculation of the sliding process of the cutting edge

Figure 9.6 A schematic representation of reacting forces in a cutting-edge sliding process...........................................

Figure 9.7 Effect of normal load from model predictions and experimental results

Figure 9.8 Effect of feature height from model predictions and experimental results

Figure 9.9 Effect of cutting-edge angle from model calculations.

Figure 9.10 Comparison of cutting-edge length with wear-particle length...... 164

Figure 9.11 Plot of particle length versus cutting-edge length................. 165

Figure 9.12 Plot of particle thickness versus penetration depth.............. 166 
Figure 9.13 Plot of particle width versus penetration depth................... 167

Figure 9.14 Plot of particle volume versus load on a log-log scale............. 168

Figure 9.15 Plot of particle volume versus sliding speed on a log-log scale..... 169

Figure 9.16 Comparison of the correlation model with the experimental results......

Figure 10.1 Estimation of particle length, particle width and particle thickness from a SEM micrograph of UHMWPE particles...

Figure 10.2 Schematic of the projected ellipsoid of a particle.................. 174

Figure 10.3 Schematic of the definition of the particle length $\left(L_{\mathrm{p}}\right)$ and the particle width $\left(W_{\mathrm{p}}\right)$ for different shapes of particles............... 176

Figure 10.4 Schematic of mechanism for particles-induced osteolysis.......... 179

Figure 10.5 Schematic particles-induced bioactivity from viewpoint of phagocytosis

Figure 10.6 Phagocytic capacity of spherical particles....................... 185

Figure 10.7 Phagocytic capacity of particles with different aspect ratios........ 186

Figure 10.8 TNF- $\alpha$ vs. index of particle internalization from Catelas et al. [79]

Figure 10.9 IL-1 $\beta$ vs. index of particle internalization from Green et al. [81].

Figure 10.10 TNF- $\alpha$ vs. Index of particle internalization from Green et al. [81].

Figure 10.11 Schematic of dynamic phagocytosis model

Figure 11.1 Schematic of murine air-pouch model for bioactivity tests of UHMWPE particles.

Figure 11.2 Micrographs of phagocytosis of UHMWPE particles by macrophage cells.

Figure 11.3 Photographs of murine air pouches; (a) without particle; (b) reacted with round-like particles; (c) reacted with elongated xvii 
particles

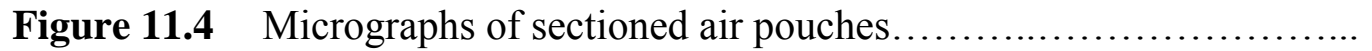

Figure 11.5 Comparison of pouch thickness for the murine air pouch tests with round-like (Group A) and elongated (Group B) UHMWPE particles.

Figure 11.6 IL-1 $\beta$ and TNF- $\alpha$ cytokine expression in murine air pouches with UHMWPE particles by real time PCR (plots A and B) and by ELISA (plots $\mathrm{C}$ and $\mathrm{D}$ ) techniques.

Figure 11.7 Comparison of pouch thickness for the murine air pouch tests with small (Group D) and larger (Group E) elongated UHMWPE particles

Figure 11.8 Comparison of pouch thickness for the murine air pouch tests with different number of particles (Group D, elongated particles within phagocytosable range)

Figure 11.9 Plot of pouch thickness versus total surface area of particles.......

Figure 11.10 Plot of pouch thickness versus total volume of particles

Figure 11.11 Plots of normalized pouch thickness versus aspect ratio of particles; (a) pouch thickness normalized by total surface area of particles; (b) pouch thickness normalized by total volume of particles. 


\section{CHAPTER 1}

\section{INTRODUCTION}

Total joint replacements have been successfully implanted clinically to replace damaged human joints for several decades. They have dramatically improved the life of patients with joint disabilities. The average life of a total hip replacement is 10 years to 15 years and the average life of a total knee replacement is less than 10 years [1]. In view of the increase of life expectancy, more active daily life style, and lower age of arthritis patients, the useful life of total joint replacements needs to be increased to reduce revision surgery at old age and the associated social and human costs if the patient cannot be operated on.

A total joint replacement system comprises of a pair of material surfaces articulating to each other to simulate the functions of the human joints. From years of research and development, an artificial joint system consisting of Co-Cr alloy sliding on an ultra-high molecular weight polyethylene (UHMWPE) surface has been proven to function well because of wear resistance, biocompatibility, and durability.

Major factors causing failure of total joint replacements include: (1) Infection during orthopedic surgery; (2) Fracture of the implants; (3) Fixation problem of the implants; (4) Wear of the implant materials; and (5) Osteolysis induced by wear particles. Of these factors, the rate of infections has been reduced by better surgery procedures. Optimizing of the material strength and minimizing its difference from the 
human bones have resolved most of the fracture problems [2-3]. The introduction of bone compatible porous surface coatings on the stem to provide another choice for fixation has helped to almost eliminate the fixation problem [4-6]. In the past 5 years, wear of UHMWPE has been dramatically reduced by using highly crosslinked UHMWPE [7-9]. The wear particle induced osteolysis has emerged as the critical failure mechanism of artificial joints.

However, the detailed mechanism of osteolysis and bone resoprtion induced by wear particles leading to the loosening of the total joint replacement is still not clear. The purpose of this study is to determine a relationship between size and shape of UHMWPE particles and bioreactivity. If the relationship between UHMWPE particle size and shape and biological responses is understood, potential engineering solutions can be developed to control the production of the most toxic size and shape of particles and hence prolong the life of the replacement joints.

To conduct this investigation, the following approaches have been adopted: (1) Generation of narrowly-distributed UHMWPE particles with different sizes and shapes; (2) Characterization of these wear particles; and (3) Bioactivity tests of UHMWPE particles in animal studies. Research staffs from NIST concentrated on developing the techniques to generate UHMWPE particles. The researchers from University of Maryland prepared the particles for animal tests. Wayne State University provided funding and research staffs to conduct the animal tests.

Various sizes and shapes of UHMWPE wear particles are needed in order to evaluate their effects on biological responses. To avoid uncertainties in the 
experimental outcomes, the uniformity of the UHMWPE particle population should be tightly controlled. A novel particle generation process by rubbing the UHWMPE material against a dimensional controlled surface texture has been developed in this study. We have proved that by varying the dimensions of the surface-texture features, the particle size and shape can be changed. By improving the uniformity of the surface texture with microfabrication techniques, the particle distribution has further been narrowed. The protocol to collect, quantify, and package particles in a sterilized environment has also been developed for particle preparation for bioactivity tests.

To understand the relationship between the surface-texture dimensions and particle dimensions, a study of the mechanism of UHWMPE particle generation has been conducted. The goal here was to formulate a mathematical model for predicting particle sizes and shapes. The desired penetration depth of a cutting edge into the material was determined from indentation tests with consideration of viscoelasticity of the UHMWPE. Simple model experiments with in-situ monitoring were conducted to observe the cutting processes and the particle generation sequence. The relationship between the tip geometry and the resultant reaction forces from the UHMWPE material during the cutting process was obtained to explain the wear particle generation mechanisms. Based on the force and energy balance of the particlegeneration process, a mathematical model was developed to predict particle volume. The model was validated by comparison with experimental results of particle generation. 
Murine air pouch animal tests were then carried out by collaboration between University of Maryland and Wayne State University to evaluate the bioactivity by applying UHMWPE particles with various sizes and shapes generated from our particle-generation processes. Related cytokine measurements and histology analyses of air pouches were performed to evaluate the degree of immunological responses induced by UHMWPE particles. Elongated wear particles were found to be much more toxic than round particles. The effects of particle size and shape on bioactivity were further analyzed from the process of phagocytosis of particles. Biophysical data of macrophage cells have been used to estimate the phagocytic capacity for various particle sizes and shapes. A critical threshold was determined and verified by our air pouch animal tests and by cell culture studies from the literature.

This study is organized in a chronological manner as follows. In Chapter 2, the backgrounds of total joint replacement, failure mechanism, wear of UHMWPE and biological responses induced by wear particles are reviewed. In Chapter 3, the current critical problem of joint replacements and approaches to solve the problems are discussed. In Chapter 4, the procedures for generating and collecting particles are described. In Chapter 5, UHMWPE particles generated from a SiC abrasive paper made steel surface are shown. The effect of surface-texture style on particle shape is discussed. Chapter 6 describes microfabrication techniques for surface-texture preparation. Chapter 7 illustrates the narrow distribution of particles generated from the microfabricated surface textures. In Chapter 8, the mechanism of particle generation from the experimental observations of worn surfaces and wear particles and 
from a simple model experiment of micro-cutting process is discussed. Chapter 9 describes mathematical models to predict the particle dimensions with given surfacetexture geometry and mechanical conditions. In Chapter 10, characterization of particles by analyzing the phagocytosis of particles is introduced. Chapter 11 introduces the air pouch animal tests of UHMWPE particles as well as the procedures for histology analysis and cytokine measurement. The effects of particle size, shape and dosage are compared in this chapter, also. In Chapter 12, the conclusions are presented. Finally, Chapter 13 mentions recommendations for future research. 


\section{CHAPTER 2}

\section{BACKGROUND}

\subsection{The need to replace human joints}

A human joint is formed by the ends of two or more bones which are connected by cartilage and tissues. For example, the hip is a ball and socket joint, formed by the upper end of the femur, the ball, and a part of the pelvis called the acetabulum, the socket. The upper end of a lower leg bone, called the tibia, and lower end of the thighbone, called the femur, form the knee joint. At the end of a joint, the bone is covered with a smooth layer of special tissue called cartilage. Normal healthy cartilage allows easy movement. Every joint is enclosed by a capsule with a smooth tissue called the synovium. The synovium produces a fluid that lubricates the joint. However, through a variety of causes, such as arthritis, infection and injury, articular cartilage can degenerate and lose its effectiveness [10]. This causes pain and stiffness in joint movement. A physical examination, laboratory tests and x-rays can determine the extent of damage. Patients suffer from chronic pain and loss of motion of the joint. The pain may be so severe that a person is inclined to avoid joint movement, weakening the muscles around the joint and making it even more difficult to move the joints. Medical treatment helps to relieve the pain caused by the damaged cartilage and helps patients to resume their normal daily life. Total joint replacement with an 
artificial joint will be prescribed when all other treatments cannot relieve pain and disability.

\section{$2.2 \quad$ Total joint replacement}

\subsubsection{Function and components}

Total joint replacement surgery is one of the most common and successful treatments for all types of arthritis and other painful conditions of the hip or knee. Joint replacement replaces destroyed or worn-out surfaces with new, uniform surfaces. For example, in an arthritic hip, the damaged ball (the upper end of the femur) is replaced by a metal ball attached to a metal stem fitted into the femur, and a plastic socket is implanted into the pelvis, replacing the damaged socket. In an arthritic knee the damaged ends of the bones and cartilage are replaced with metal and plastic surfaces that are shaped to simulate knee movement and function. Although hip and knee replacements are the most common, joint replacements can be performed on other joints, including the ankle, foot, shoulder, elbow and fingers.

The materials used in a total joint replacement are designed to enable the joint to move smoothly with low friction $[11,12]$. A joint implant is generally composed of two parts that articulate during the joint motion. In a hip, for example a metal ball is usually used to fit closely into a matching counter surface composed of UHMWPE. Several metals are used, including stainless steel, alloys of cobalt and chrome, and titanium. The UHMWPE is durable and wear resistant. It has been widely accepted for its low friction and high wear-resistance. Examples of total hip and knee replacements 
with Co-Cr alloy and UHMWPE parts are shown in Figure 2.1. While these materials are mostly used, combinations of ceramic-ceramic, metal-metal and ceramicUHMWPE total joint systems are some other alternative materials used under special situations [13-17]. Bone cements made by polymers are usually used to anchor the prosthesis into the bone [18-19]. Joint replacements also can be implanted without cement when a porous surface on the stems of joint replacements is proved to allow bone to grow into it.

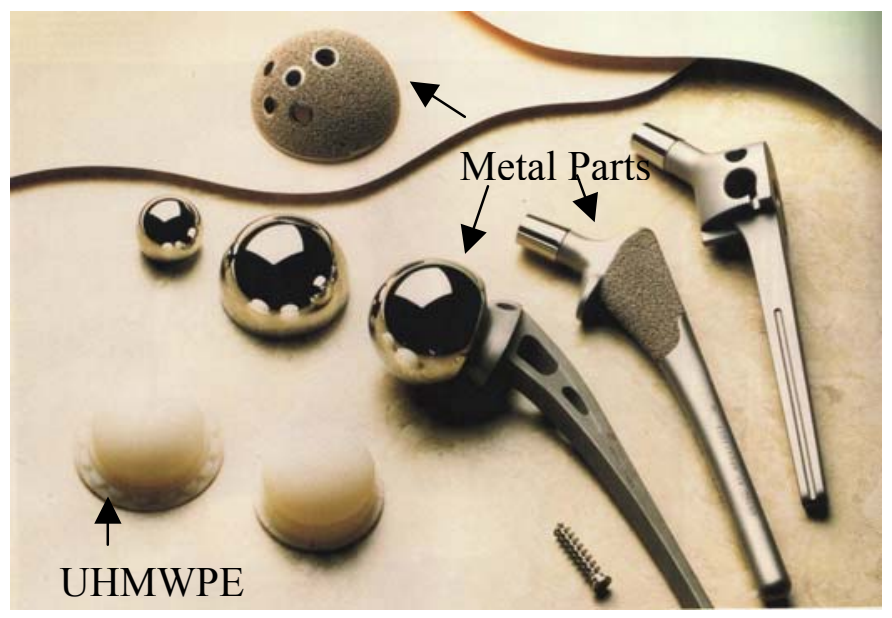

Components of total hip replacements

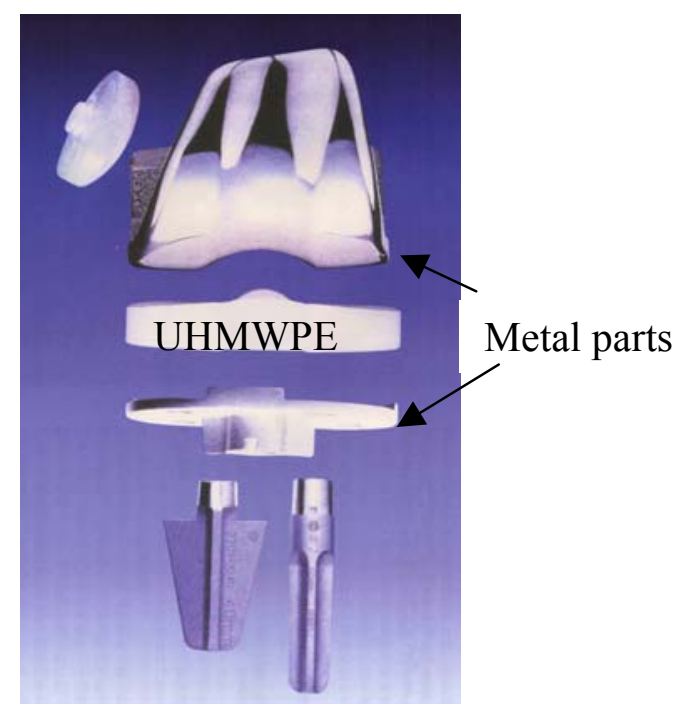

Components of total knee replacement

Figure 2.1 Components of total hip and knee joint replacements. 


\subsubsection{Material properties of UHMWPE and their implications}

Ultra-high molecular weight polyethylene is widely used as the bearing material in total hip and knee replacements. Polyethylene has the repeating unit chemical structure:

$$
-\left(\mathrm{CH}_{2}-\mathrm{CH}_{2}\right)_{n}-
$$

There are several kinds of polyethylene (LDPE, HDPE, UHMWPE) that are synthesized with different relative molecular mass and chain architectures. LDPE refers to low-density polyethylene with a relative molecular mass of less than 50,000 $\mathrm{M}_{\mathrm{r}}$. High-density polyethylene (HDPE) is a linear polymer with a relative molecular mass up to $200,000 \mathrm{M}_{\mathrm{r}}$. In comparison, UHMWPE has a relative molecular mass ranging from (2 to 6) $\times 10^{6} \mathrm{M}_{\mathrm{r}}$ [20]. The typical modern UHMWPE used in implants has a relative molecular mass of (4 to 6$) \times 10^{6} \mathrm{M}_{\mathrm{r}}$. Table 2.1 summaries the physical and mechanical properties of HDPE and UHMWPE. UHMWPE has a higher ultimate strength and impact strength than HDPE and UHMWPE is significantly more abrasion and wear resistant than HDPE from a clinical perspective [21].

Table 2.1 Typical physical properties of HDPE and UHMWPE [21].

\begin{tabular}{|l|l|l|}
\hline Property & HDPE & UHMWPE \\
\hline Relative Molecular Mass $\left(\mathrm{M}_{\mathrm{r}}=\mathrm{g} / \mathrm{mol}\right)$ & 0.05 to 0.25 & 2 to 6 \\
\hline Melting Temperature $\left({ }^{\circ} \mathrm{C}\right)$ & 130 to 137 & 125 to 138 \\
\hline Poisson's Ratio & 0.40 & 0.46 \\
\hline Specific Gravity & 0.952 to 0.965 & 0.932 to 0.945 \\
\hline Tensile Modulus of Elasticity (GPa) & $0.4-4.0$ & 0.8 to 1.6 \\
\hline Tensile Yield Strength (MPa) & 26 to 33 & 21 to 28 \\
\hline Tensile Ultimate Strength (MPa) & 22 to 31 & 39 to 48 \\
\hline Tensile Ultimate Elongation (\%) & 10 to 1200 & 350 to 525 \\
\hline Degree of Crystallinity $(\%)$ & 60 to 80 & 39 to 75 \\
\hline
\end{tabular}


The molecular chain of UHMWPE can be visualized as a tangled string of wires. The chain is not static and its arrangement is a function of temperature. The molecular chain becomes mobile at elevated temperatures. When the temperature is lower than the melting temperature, the molecular chain of UHMWPE tends to rotate along the C-C bonds and create chain folds. The chain folding leads to an ordered arrangement of molecules so as to form a crystalline region [22]. A schematic of the crystalline region with folded chains and amorphous region with loose chains fused together is shown in Figure 2.2. The degree and orientation of the crystalline regions depends on its molecular weight, processing conditions and environment conditions.

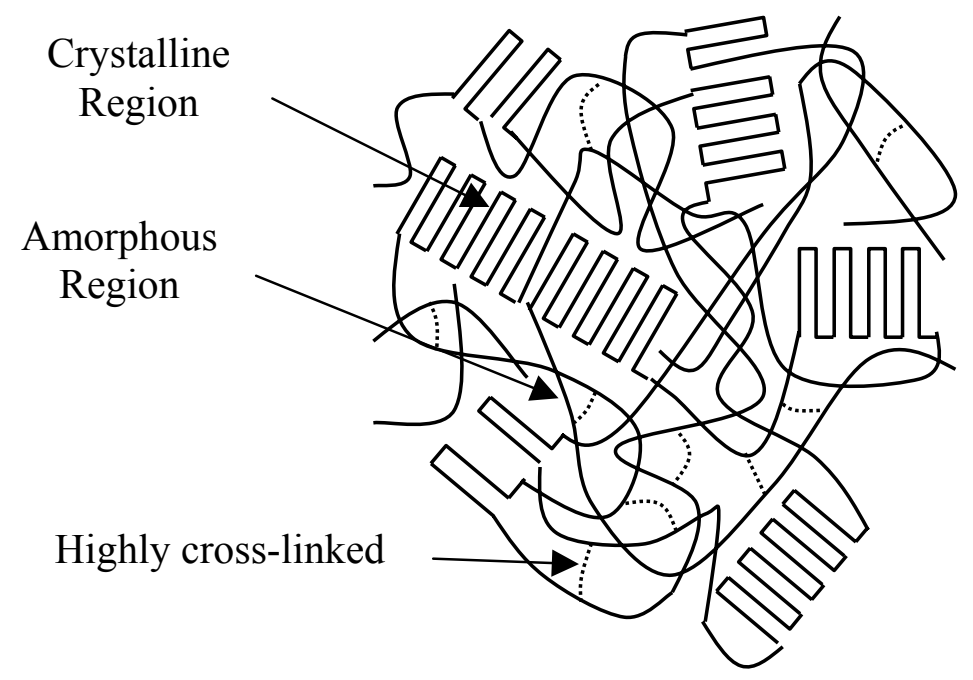

Figure 2.2 Schematic diagram of the chain orientation of UHMWPE. 
To be used for medical applications, UHMWPE resin powder should meet the requirements specified in ASTM standard F648 [23] and ISO standard 5834-1 [24]. UHMWPE is produced as powder and must be consolidated under high temperature and pressure by various thermoplastic-processing techniques, such as compression molding and ram extrusion [25]. Then the material is machined into its final form. The UHMWPE components for total joint replacement are then packaged and sterilized before distribution for medical use.

The material properties of UHMWPE are sensitive to the manufacturing processes, post-treatment of the material, and material-storage environments. For example, when a shear force is applied to the material surface, the molecules tend to align in the direction of shear stress [26-28], and the surface material strength may be strongly dependent on direction of shear motions [29]. It has been shown that the wear of UHMWPE is accelerated by the cross-shear that occurs [74].

Another factor is the sterilization process of the material. After the manufacturing of artificial joint components, the parts will go through a sterilization process before being implanted into the human body. The conventional sterilization technique by steam autoclaving cannot be used with polyethylene components because of their tendency to deform under this condition. Sterilization by gamma irradiation is normally used. However, this causes the polyethylene to cross-link, which in turn, modifies the polymer molecular weight, crystallinity, elastic modulus and other physical properties [30]. 


\subsection{Failure of total joint replacement}

The long-term performance of the articulating system and materials in the total joint replacement is very important, in particular for younger, heavier, and more active patients. It has been established that the failure of total joint replacement can be attributed to one of the following causes: 1) Infection from surgery, 2) Fracture of implants, 3) Inability to obtain suitable fixation, 4) Wear of UHMWPE, and 5) Osteolysis induced by UHMWPE wear particles. Wear-particle induced aseptic loosening is recognized as a major cause leading to failure of total joint replacements [31-34]. In the following sections, more details are presented related to wear particles of UHMWPE and osteolysis induced by such wear particles.

\subsubsection{Wear and wear particles of UHMWPE components}

Ultra-high molecular weight polyethylene (UHMWPE) is identified as one the major components of wear for orthopedic joint implants. Figure 2.3 shows examples of worn UHMWPE parts observed from total hip and knee replacements. UHMWPE wear particles are generated around the joint region due to the articulation motion between the metal parts and UHMWPE components. It has been suggested that various wear particles are embedded among the prosthetic tissues or synovial fluid around the failed artificial joints. Many studies have isolated UHMWPE particles from the tissues retrieved from the revision surgery [35-46]. Table 2.2 provides a summary of retrieval UHMWPE wear particle studies in the literature. The table also lists the 
digestion procedure for isolating the UHMWPE particles, identification of materials and analysis of particle size and shape.

Size, shape and morphology of UHMWPE wear particles have been widely analyzed [47-51]. It has been observed that the size range of UHMWPE wear particles are from sub-micrometers to millimeters. A variety of particle shapes have been observed: granules, beads, fibrils, shreds. The morphology of the particles is asymmetric and irregular. Examples of particles retrieved from total knee replacement (TKR) are shown in Figure 2.4.

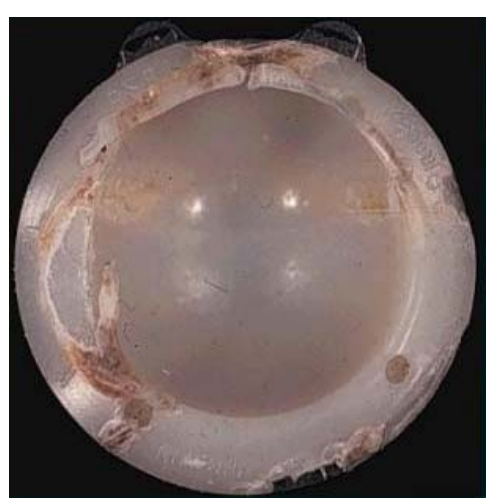

Worn UHMWPE cup of total hip replacement.

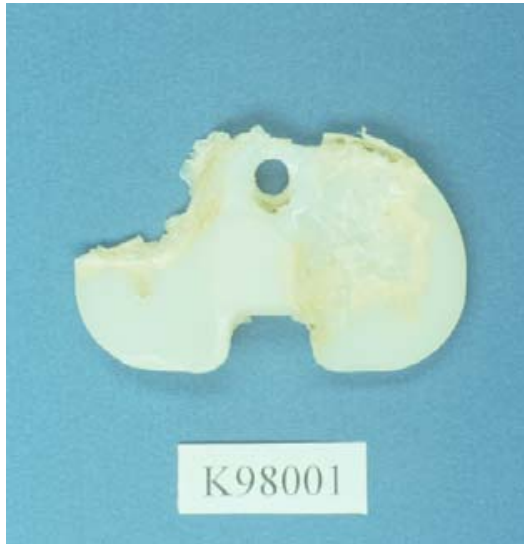

Worn UHMWPE component of total knee replacement.

Figure 2.3 Examples of worn UHMWPE components for total hip and knee replacements. 
Table 2.2 Summary of retrieval UHMWPE wear particle studies in the literature.

\begin{tabular}{|c|c|c|c|c|}
\hline Reference & $\begin{array}{l}\text { Sample } \\
\text { source }\end{array}$ & Particle isolation & $\begin{array}{c}\text { Material } \\
\text { analysis }\end{array}$ & Particle analysis \\
\hline $\begin{array}{l}\text { Wolfarth et al. [35] } \\
\text { JBMR, vol. 34, pp.57-61 } \\
\text { (1997) } \\
\text { *FDA }\end{array}$ & $\begin{array}{l}\text { Synovial } \\
\text { fluid, } \\
\text { tissues } \\
\text { (TKR) }\end{array}$ & $\begin{array}{l}\mathrm{NaOH} \text { digestion, ultra- } \\
\text { centrifugation, water } \\
\text { and ethanol } \\
\text { centrifugation }\end{array}$ & $\begin{array}{l}\text { Micro- } \\
\text { Ramman } \\
\text { spectroscopy }\end{array}$ & $\begin{array}{l}\text { SEM } \\
\text { The particles from synovial fluid } \\
\text { and tissue are not much different. } \\
\text { Smooth, granular shapes, thin, } \\
\text { irregularly-shaped, shaving like } \\
\text { particles. }\end{array}$ \\
\hline $\begin{array}{l}\text { Campbell et al. [36] } \\
\text { JBMR, vol. 29, pp. 127-131 } \\
\text { (1995) } \\
\text { *LA orthopedic hospital }\end{array}$ & \begin{tabular}{|l} 
Tissues \\
(THR)
\end{tabular} & $\begin{array}{l}\mathrm{NaOH} \text { digestion, } \\
\text { density gradient ultra- } \\
\text { centrifugation, sucrose } \\
\text { density gradient } \\
\text { centrifugation }\end{array}$ & FTIR, DSC & $\begin{array}{l}\text { SEM } \\
* \text { Rounded }(0.3 \mu \mathrm{m}) \\
* \text { Elongated }(1.9 \mu \mathrm{m}) \\
* \text { Fibrils with rounded heads at } \\
\text { one end, thin taper at the other }\end{array}$ \\
\hline $\begin{array}{l}\text { Shanbhag et al. [37] } \\
\text { JBJS, vol. 76-B, pp. 60-67 } \\
\text { (1994) } \\
\text { *Rush-Presbyterian-St } \\
\text { Luke's medical center }\end{array}$ & $\begin{array}{l}\text { Tissues, } \\
\text { interfacial } \\
\text { membran } \\
\text { es (THR) }\end{array}$ & $\begin{array}{l}\text { KOH digestion, } \\
\text { centrifugation, hexane- } \\
\text { ethanol fractions }\end{array}$ & FTIR, EDX & $\begin{array}{l}\text { SEM } \\
* \text { Spheroids: } 0.1 \mu \mathrm{m} \text { to } 0.2 \mu \mathrm{m} \\
\text { * Fibrils: } 0.2 \mu \mathrm{m} \text { to } 0.3 \mu \mathrm{m} \text { wide, } \\
\text { up to } 10 \mu \mathrm{m} \text { long. } \\
* 92 \% \text { particles are smaller than } \\
1 \mu \mathrm{m} \text {. }\end{array}$ \\
\hline $\begin{array}{l}\text { Hailey et al. [38] } \\
\text { PIME, vol. } 210, p p .3-10 \\
\text { *U. of Leeds, UK }\end{array}$ & $\begin{array}{l}\text { Tissue } \\
\text { (THR) }\end{array}$ & $\begin{array}{l}\text { KOH digestion, } \\
\text { centrifugation, } 0.2 \mu \mathrm{m} \\
\text { filtration }\end{array}$ & $\mathrm{N} / \mathrm{A}$ & $\begin{array}{l}\text { SEM } \\
0.3 \mu \mathrm{m} \text { up to } 3 \mathrm{~mm}\end{array}$ \\
\hline $\begin{array}{l}\text { Lee et al. [39] } \\
J B J S, \text { vol. } 74-B, p p .380-384 \\
(1992) \\
\text { *Hospital for special } \\
\text { surgery, NY }\end{array}$ & $\begin{array}{l}\text { Tissues } \\
\text { (THR) }\end{array}$ & $\begin{array}{l}\text { Digestion in tissue } \\
\text { solubiliser, } \\
\text { centrifugation }\end{array}$ & $\mathrm{N} / \mathrm{A}$ & $\begin{array}{l}\text { Polarized light microscopy } \\
\text { Short dimension } 2 \mu \mathrm{m} \text { to } 4 \mu \mathrm{m} \text {, } \\
\text { long dimension } 8 \mu \mathrm{m} \text { to } 13 \mu \mathrm{m} \text {. }\end{array}$ \\
\hline $\begin{array}{l}\text { Maloney et al. [40] } \\
J B J S, \text { vol. 77-A, pp. 1301- } \\
\text { 1310 (1995) } \\
\text { *Stanford U. }\end{array}$ & $\begin{array}{l}\text { Tissues } \\
\text { (THR) }\end{array}$ & Papain digestion & X-ray & $\begin{array}{l}\text { SEM, Coulter multisizer II } \\
\text { * Fixed cup: } 0.4 \mu \mathrm{m} \\
* \text { Bipolar: } 0.7 \mu \mathrm{m}\end{array}$ \\
\hline $\begin{array}{l}\text { Schmalzried et al. [41] } \\
\text { JBMR, vol. 38, pp. 203-210 } \\
\text { (1997) } \\
\text { *LA orthopedic hospital }\end{array}$ & $\begin{array}{l}\text { Tissues } \\
\text { (THR, } \\
\text { TKR) }\end{array}$ & $\begin{array}{l}\text { NaOH digestion, } \\
\text { sucrose ultra- } \\
\text { centrifugation, } \\
\text { isopropanol density } \\
\text { gradient ultra- } \\
\text { centrifugation }\end{array}$ & FTIR, DSC & $\begin{array}{l}\text { SEM } \\
* \text { Granules: sub-micron } \\
* \text { Beads: } 1 \mu \mathrm{m} \text { to } 2 \mu \mathrm{m} \\
* \text { Fibrils: up to } 5 \mu \mathrm{m} \\
\text { * Shreds: } 10 \mu \mathrm{m} \text { to } 20 \mu \mathrm{m} \text { long, } \\
\text { up to several microns wide. }\end{array}$ \\
\hline $\begin{array}{l}\text { Hirakawa et al. [42] } \\
J B M R, \text { vol. 31, pp. 257-263 } \\
\text { (1996) } \\
\text { *The Cleveland clinic } \\
\text { foundation }\end{array}$ & $\begin{array}{l}\text { Tissues } \\
\text { (THR, } \\
\text { TKR) }\end{array}$ & $\begin{array}{l}70 \% \text { Nitric acid } \\
\text { digestion, } \\
\text { centrifugation, } 0.4 \mu \mathrm{m} \\
\text { filtration }\end{array}$ & EDX & $\begin{array}{l}\text { SEM, Coulter multisizer II } \\
*<10 \mu \mathrm{m} \text { particles: } 0.72 \mu \mathrm{m} \\
\text { (hip), } 0.74 \text { (knee) } \\
*>10 \mu \mathrm{m} \text { particles: } 81 \mu \mathrm{m} \text { (hip), } \\
283 \mu \mathrm{m} \text { (knee) }\end{array}$ \\
\hline
\end{tabular}




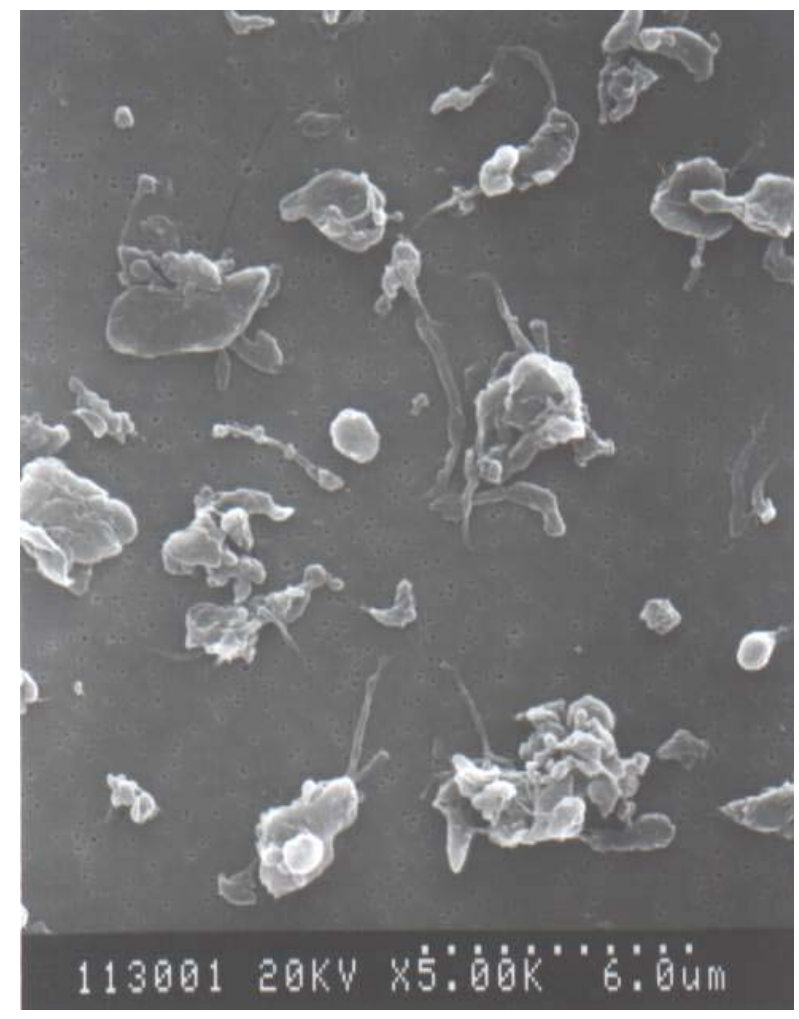

Figure 2.4 Examples of UHMWPE particles retrieved from total knee replacement. 


\subsubsection{Wear particles induced osteolysis}

Osteolysis that causes the loosening of orthopedic joint replacements is recognized as the major problem leading to the failure of the implants. The region of osteolysis has a lower bone density and can be detected by X-ray photograph as shown in Figure 2.5. Macrophage phagocytosis of wear particles and the consequent immunological responses, including the production of bone resorptive cytokines and activation of osteoclast cells are the major causes of the osteolysis. Table 2.3 provides a list of definitions of biological terms helpful for understanding the mechanism mentioned below. From the articulation between metal and UHMWPE parts, wear particles have been generated and embedded among surrounding tissues [52-54]. Macrophage cells act as the first defensive line to protect the host. Macrophage cells are activated by wear particles as foreign substances and then engulf and digest the particles [55]. The process is called phagocytosis of wear particles by macrophage cells. It is believed that numerous of inflammatory products, including TNF- $\alpha$, IL-1 $\beta$, and IL-6 cytokines, are released during the process [56-57]. Osteoblasts are stimulated by those mediators to produce osteoclast promoting substances, like GM-CSF, IL-6, and PGE-2 [56-57]. Osteoclast cells are finally activated and these resorb bone cells surrounding the artificial joint insertion area. The phenomenon is called bone resorption and osteolysis [52-53]. 
Table 2.3 Definition of biological terms related to osteolysis

\begin{tabular}{|c|l|}
\hline Terms & \multicolumn{1}{|c|}{ Definition } \\
\hline Osteolysis & $\begin{array}{l}\text { Dissolution of bone through disease, commonly due to infection or by } \\
\text { loss of blood supply to the bone. }\end{array}$ \\
\hline Necrosis & $\begin{array}{l}\text { The death of some of all the cells in an organ or tissue, caused by } \\
\text { disease, physical or chemical injury, or interference with the blood } \\
\text { supply. }\end{array}$ \\
\hline Phagocytosis & $\begin{array}{l}\text { The engulfment and digestion of bacteria and other foreign particles by } \\
\text { a cell. }\end{array}$ \\
\hline Macrophage & $\begin{array}{l}\text { A large scavenger cell (a phagocyte) present in connective tissue and } \\
\text { many major organs and tissues, including the bone marrow, spleen, } \\
\text { lymph nodes, liver, and the central nervous system. }\end{array}$ \\
\hline Osteoblast & $\begin{array}{l}\text { A cell, originating in the mesoderm of the embryo that is responsible } \\
\text { for the formation of bone. }\end{array}$ \\
\hline Osteoclast & $\begin{array}{l}\text { A large multinucleate cell that resorbs calcified bone. Osteoclasts are } \\
\text { only found when bone is being resorbed and may be seen in small } \\
\text { depressions on the bone surface. }\end{array}$ \\
\hline Cytokine & $\begin{array}{l}\text { Soluble mediators secreted by macrophages, controlling many critical } \\
\text { interactions among cells of the immune system. }\end{array}$ \\
\hline IL-1 $\beta$, IL-6 & Cytokine, interleukins mediators \\
\hline TNF- $\alpha$ & Cytokine, tumor necrosis factor $\alpha$ \\
\hline GM-CSF & Cytokine, colony-stimulating factors \\
\hline PGE-2 & Cytokine, prostaglandins \\
\hline
\end{tabular}

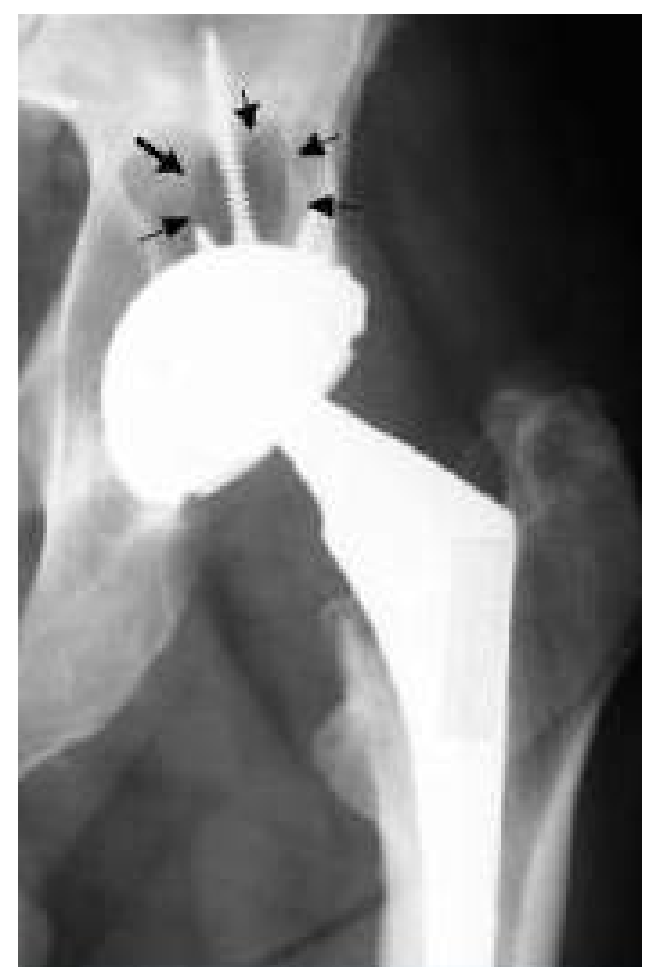

Figure 2.5 Osteolysis taking place around the total hip replacements which is detected by X-ray diagnosis. 


\subsection{Wear studies of UHMWPE material}

To simulate the wear of total joint replacement in the human body, wear studies of UHMWPE have been performed in bovine serum at ranges of contact pressure and sliding speed encountered in real situations [58-66]. Scanning electron microscopy results have demonstrated that surface damages of UHMWPE include adhesion wear, abrasion, and delamination wear. The contributions of these mechanisms to the overall wear rate and the generation of wear particles depend predominantly on the contact pressure and secondarily on the sliding speed. Transmission electron microscopy has provided the microstructural morphology of UHMWPE obtained from sliding: alignment of crystalline regions in the polyethylene microstructure parallel to the sliding surface was found to occur during sliding even at low contact pressures. The worn polymer surface and debris have been extensively characterized for structural changes with X-ray diffraction and differential scanning calorimetry.

Friction and wear behavior depend on many parameters including contact geometry, types of contact materials, the quality of surface, and lubrication. It has been shown [59-61] that lubrication of the artificial hip joint is complicated and that long-term performance is governed by the combined wear, creep, and oxidation degradation of the articulating materials. More importantly, it has been shown that a tendency for heating exists during articulation in the hip joint and that elevated temperatures could increase the wear, creep, and oxidation degradation rate of UHMWPE. 
In order to investigate the clinical wear performance of UHMWPE, we need to understand the biomechanics of the total joint replacement. The force transmitted to the hip-joint implant during gait has been measured [67-72]. Figure 2.6 shows an example of the forces acting on a hip-joint implant during gait [67]. It is shown to exhibit a double-hump shape of the force curve. The maximum force acting on the joint implant is as high as $300 \%$ body weight of a person. For a total hip replacement, the articulation of the artificial joints consists of three principal components: flexion/extension, abduction/adduction and femoral rotation. Gait analyses have shown that the motion of the hip joint differs from person to person. However, the three basic components of motion are common to most people. The hip-joint kinematics of a group of people during normal gait has been studied [73] and it has been found that the average levels of flexion/extension, abduction/adduction, and femoral rotation were $+40^{\circ} /-3^{\circ},+7.5^{\circ} / 5^{\circ}$ and $+5^{\circ} / 5^{\circ}$, respectively. The trajectories of contact points on the articular surface of the hip-joint implant per gait cycle were plotted by Wang et al. [74] as shown in Figure 2.7. It is seen that each point follows a different open loop. Cross-overs of the loops occur everywhere over the entire surface of contact. The human joint motion is a multi-directional as are the surface shear stresses. 


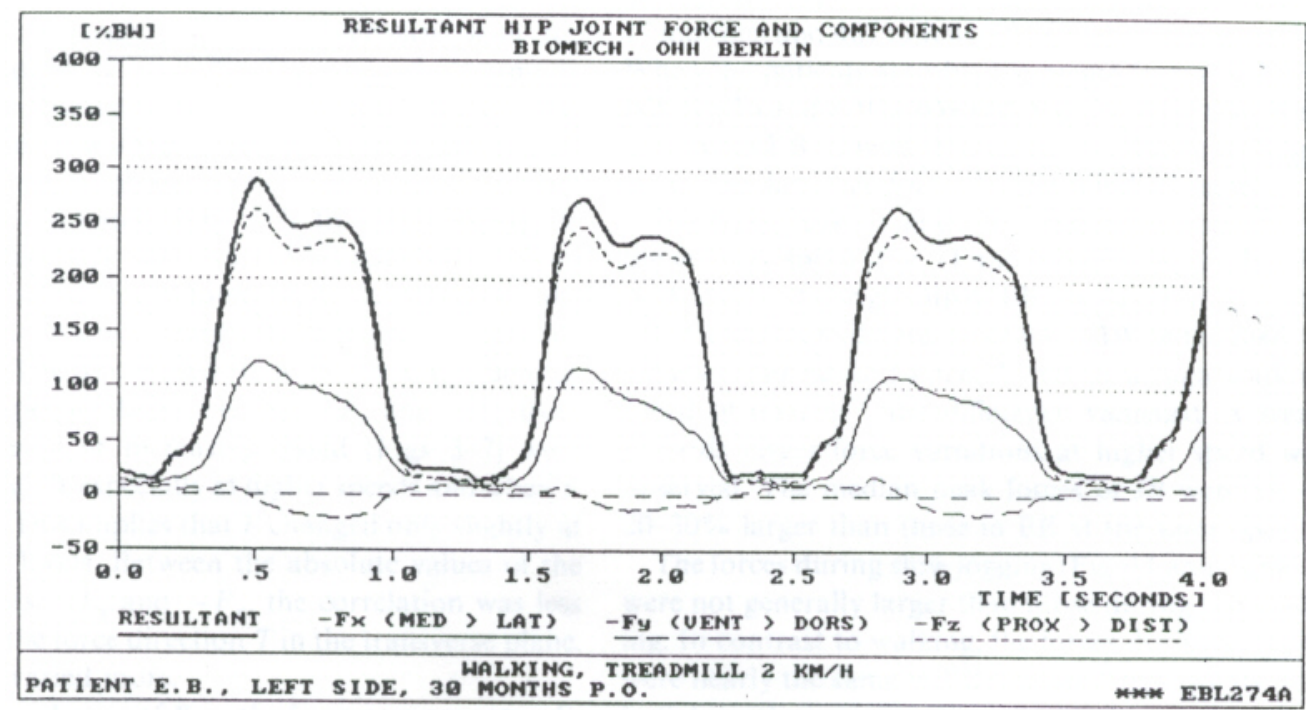

Figure 2.6 An example of forces acting on the hip-joint implant during gait [67].

Contact paths on the hip joint surface

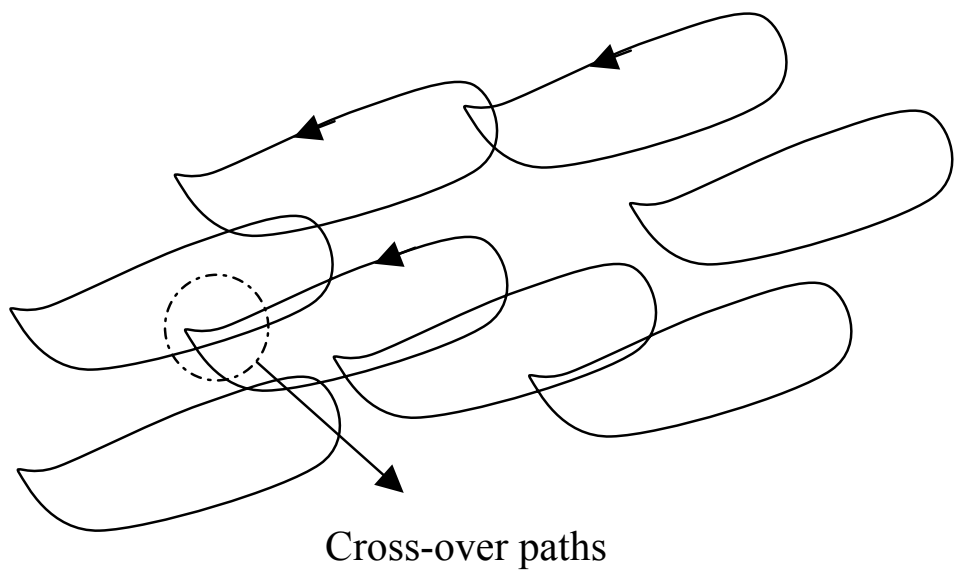

Figure 2.7 Trajectories of contact points on the articular surface of hip-joint implant. 
The mechanism of wear and wear particle generation of UHMWPE in a hipjoint implant can be described as follows [74]: (a) joint articulation creates a multidirectional stress field at the wear surface; (b) the principal stress component occurs in the direction of flexion and extension; (c) the secondary stress component occurs in the direction of abduction/adduction for the hip and in the direction of tibial rotation for the knee; (d) preferential orientation of molecules occurs in the direction of the principal stress component; (e) orientation leads to strain softening in the transverse direction which coincides with the secondary stress component; (f) transverse softening leads to surface rupture between the oriented molecules, and (g) surface rupture leads to transfer of load to the subsurface shear plan which results in shear rupture and the formation of UHMWPE wear particles. The size and shape of UHMWPE wear- particles are determined from the above processes.

Studies have also indicated that screening wear machines based on linear motion do not correlate with multi-axial joint simulators and may produce misleadingly low wear results [75-77]. Many researchers have implemented multidirectional motion wear testing to evaluate the clinical performance of UHMWPE in a bench testing system [75-77]. The motion direction has been shown as a key factor for accelerated wear of UHMWPE material. 


\subsection{Evaluation of Bioactivity of UHMWPE Particles}

\subsubsection{Wear-particle induced reactions and mechanisms}

Osteolysis and bone resorption is the major failure of total joint replacement. However, at present, there is no medical or pharmacologic treatment for this problem. The precise mechanism of the bone loss remains to be established. Several studies have indicated the breakdown of the artificial joint materials and that release of the wear particles may be a critical factor. Evaluation of tissue from the interface between implant and bone taken from the aseptically loose total joint implant has demonstrated the presence of macrophages phagocytosing particles in association with areas of bone resorption. It has been postulated that macrophage phagocytosis of wear particles may induce several inflammotary mediators stimulating bone resoprtion. To further investigate the effect of UHMWPE particles on bioactivity, cell-culture tests and animal tests are two major experimental approaches. Table 2.4 gives a summary of bioactivity tests from the literature [78-87]. Cell-culture studies and animal tests will be further discussed in the following sections. 
Table 2.4 Summary of bioactivity study of UHMWPE particles from literature

\begin{tabular}{|c|c|c|c|}
\hline \begin{tabular}{|l|} 
Reference \\
\end{tabular} & Experiment & PE particles & \begin{tabular}{|l|} 
Noted effect \\
\end{tabular} \\
\hline $\begin{array}{l}\text { Allen et al. [78] } \\
\text { JBJS, vol. 78-B, pp. } \\
\text { 32-37 (1996) } \\
\text { *U. of Cambridge } \\
\text { Ortho. Res. Unit }\end{array}$ & $\begin{array}{l}\text { In-vivo, rats at } \\
\text { weight bearing } \\
\text { bone-implant } \\
\text { interface }\end{array}$ & HDPE: round, $2.03 \mu \mathrm{m}$ & $\begin{array}{l}\text { Causing chronic inflammatory response } \\
\text { with numerous foreign-body giant cells } \\
\text { in peri-prosthetic tissues }\end{array}$ \\
\hline $\begin{array}{l}\text { Catelas et al. [79] } \\
J B M R, \text { vol. 41, pp. } \\
600-607 \text { (1998) } \\
\text { *Ecole } \\
\text { polytechnique de } \\
\text { Montreal }\end{array}$ & $\begin{array}{l}\text { In-vitro, } \\
\text { macrophage cell } \\
\text { culture }\end{array}$ & HDPE: round, $4.5 \mu \mathrm{m}$ & $\begin{array}{l}* \text { TNF- } \alpha \text { release } \\
* \text { Cell viability decreased }\end{array}$ \\
\hline $\begin{array}{l}\text { Goodman et al. [80] } \\
\text { Biomaterials, vol. } \\
\text { 17,pp. 1943-1947 } \\
\text { (1996) } \\
\text { * Stanford U. } \\
\text { medical center }\end{array}$ & $\begin{array}{l}\text { In-vivo, rabbits } \\
\text { through a drill hole } \\
\text { into proximal tibia }\end{array}$ & HDPE: round, $(4.7 \pm 2.1) \mu \mathrm{m}$ & $\begin{array}{l}10^{6} \text { to } 10^{9} \text { particles } / \mathrm{ml} \text { evoked } \\
\text { histiocytic reaction }\end{array}$ \\
\hline $\begin{array}{l}\text { Green et al. [81] } \\
\text { Biomaterials, vol. } \\
\text { 19, pp. 2297-2302 } \\
\text { (1998) } \\
\text { * U. of Leeds, UK }\end{array}$ & $\begin{array}{l}\text { In-vitro, } \\
\text { macrophage cell } \\
\text { culture }\end{array}$ & $\begin{array}{l}\text { * UHMWPE (Ceridust 3615): } \\
(0.21 \pm 0.069) \mu \mathrm{m},(0.49 \pm 0.11) \\
\mu \mathrm{m},(4.3 \pm 1.89) \mu \mathrm{m}, \\
(7.2 \pm 3.15) \mu \mathrm{m} \\
\text { * UHMWPE (GUR 1120): } \\
(88 \pm 29) \mu \mathrm{m}\end{array}$ & $\begin{array}{l}\text { * Elevated level of TNF- } \alpha \text { and IL-1 } \beta \text { : } \\
\text { * Elevated level of IL-6: } \\
\text { * Size and volume (number) are critical } \\
\text { factors in macrophage activation. }\end{array}$ \\
\hline $\begin{array}{l}\text { Horowitz et al. [82] } \\
\text { J. Ortho. Res., vol. } \\
\text { 15, pp. 50-56 (1997) } \\
\text { * Hospital of U. of } \\
\text { Penn. }\end{array}$ & $\begin{array}{l}\text { In-vitro, } \\
\text { macrophage/ } \\
\text { osteoblasts co- } \\
\text { culture }\end{array}$ & HDPE: round, $(5.4 \pm 0.3) \mu \mathrm{m}$ & $\begin{array}{l}* \mathrm{TNF}-\alpha \text { and } \mathrm{PGE}_{2} \text { release } \\
* \text { Bone resorption }\end{array}$ \\
\hline $\begin{array}{l}\text { Howie et al. [83] } \\
\text { JBJS, vol. 70-A, pp. } \\
\text { 257-263 (1988) } \\
\text { * Royal Adelaide } \\
\text { Hospital }\end{array}$ & $\begin{array}{l}\text { In-vivo, rat at } \\
\text { cement-bone } \\
\text { interface }\end{array}$ & $\begin{array}{l}\text { UHMWPE (Howmedica): } \\
\text { irregular, 20-200 } \mu \mathrm{m}\end{array}$ & Resorption of bone occurred. \\
\hline $\begin{array}{l}\text { Matinez et al. [84] } \\
\text { Biomaterials, vol. } \\
\text { 19, pp. 183-187 } \\
\text { (1998) } \\
\text { * Paseo de la } \\
\text { Castellana, Spain }\end{array}$ & $\begin{array}{l}\text { In-vitro, human- } \\
\text { osteoblastic cell } \\
\text { culture }\end{array}$ & $\begin{array}{l}\text { * UHMWPE (Hoechst): }<80 \\
\mu \mathrm{m} \\
* \text { UHMWPE (Howmedica): } \\
<100 \mu \mathrm{m}\end{array}$ & $\begin{array}{l}\text { PE particles inhibit human osteoblastic } \\
\text { cell growth. }\end{array}$ \\
\hline $\begin{array}{l}\text { Murray et al. [85] } \\
\text { JBJS, vol. 72-B, pp. } \\
988-992 \text { (1990) } \\
\text { * Nuffield } \\
\text { orthopaedic center, } \\
\text { UK }\end{array}$ & $\begin{array}{l}\text { In-vitro, } \\
\text { macrophage cell } \\
\text { culture }\end{array}$ & $\begin{array}{l}\text { HDPE: } \approx 1 \mu \mathrm{m} \text { ( } 1200 \text { grit } \\
\text { silicon carbide paper } \\
\text { fabricated) }\end{array}$ & $\begin{array}{l}* \text { Phagocytosis of particles } \\
* \text { Release of } \mathrm{PGE}_{2} \rightarrow \text { osteoclastic bone } \\
\text { resorption }\end{array}$ \\
\hline $\begin{array}{l}\text { Shanbhag et al. [86] } \\
\text { JBMR, vol. 28, pp. } \\
\text { 81-90 (1994) } \\
\text { * Rush-Presbyterian- } \\
\text { St. Luke's medical } \\
\text { center [25] }\end{array}$ & $\begin{array}{l}\text { In-vitro, } \\
\text { macrophage cell } \\
\text { culture }\end{array}$ & $\begin{array}{l}\text { UHMWPE (GUR 415) } \\
*(0.47 \pm 0.2) \mu \mathrm{m} \text { (retrieved) } \\
*(0.66 \pm 0.6) \mu \mathrm{m} \text { (fabricated) }\end{array}$ & $\begin{array}{l}* 10 \mathrm{x} \text { surface ratio to stimulate bone } \\
\text { resorption } \\
* \text { Nonosteolytic chronically tolerable } \\
\text { dose: } 1 \mathrm{x} \text { surface ratio }\end{array}$ \\
\hline $\begin{array}{l}\text { Voronov et al. [87] } \\
\text { JBMR, vol. 39, pp. } \\
40-51 \text { (1998) } \\
\text { * U. of Toronto [26] }\end{array}$ & $\begin{array}{l}\text { In-vitro, } \\
\text { macrophage cell } \\
\text { culture }\end{array}$ & $\begin{array}{l}\text { * UHMWPE: } 18 \mu \mathrm{m} \text { to } 20 \\
\mu \mathrm{m} \\
* \text { HDPE: } 4 \mu \mathrm{m} \text { to } 10 \mu \mathrm{m}\end{array}$ & $\begin{array}{l}\text { * Smaller particles were phagocytosed } \\
\text { * Release IL-1 } \beta, \text { IL-1 } \alpha, \text { TNF- } \alpha \text { and } \\
\text { PGE }_{2}\end{array}$ \\
\hline
\end{tabular}




\subsubsection{In-vitro cell culture study}

Many researchers have set up in-vitro cell-culture tests to study the effect of polyethylene particles. Catelas et al. [79] applied HDPE particles in an in-vitro macrophage cell-culture test and observed an increase of TNF- $\alpha$ release and a decrease of cell viability in the system. Horowitz et al. [82] set up an in-vitro macrophage/osteoblast co-culture system to study the stimulation response of HDPE particles and they observed bone resorption. Vornov et al. [87] compared commercial HDPE and UHMWPE particles with different sizes in a macrophage cell-culture study. Martinez et al. [84] obtained different UHMWPE particles with different size ranges and applied them in an in-vitro human-osteoblastic cell-culture test. It was concluded that human osteoblastic cell growth was inhibited due to the presence of UHMWPE particles. Green et al. [81] applied particles collected from wear tests in a macrophage cell-culture study; from the measurement of IL-1 $\beta$ and TNF- $\alpha$ releases, they concluded that the size and volume of particles are critical factors in macrophage activation. Shanhbag et al. [86] used particles, prepared by an attrition process, in an in-vitro macrophage cell-culture study and determined the critical particle to macrophage cell-surface ratio to stimulate bone resorption.

\subsubsection{In-vivo animal tests}

In-vivo animal tests of particles can mimic a suitable environment for evaluating biological response induced by polyethylene particles. Allen et al. [78] injected HDPE particles into rats at the weight-bearing bone-implant interface. A 
chronic inflammatory response with numerous foreign-body giant cells in periprosthetic tissues was observed. Goodman et al. [80] have put HDPE particles through a drill hole into proximal tibia in a rabbit and found a histiocytic reaction after a critical particle concentration was reached. Howie et al. [83] placed commercial UHMWPE particles at the cement-bone interface in a rat and resorption of bone occurred. It was found that smaller particles were phagocytosed with elevation of IL$1 \beta$, TNF- $\alpha$, and $\mathrm{PGE}_{2}$.

Current understanding of the particles induced bioactivity can be summarized as follows:

(1) From cell culture and animal studies, a significant elevated level of TNF- $\alpha$, IL$1 \beta$, and IL- 6 cytokines has been measured. Most studies have indicated that phagocytosis of polyethylene by macrophage cells stimulated the release of related cytokines.

(2) Several studies have shown the effects of the round-like UHMWPE particle size and concentration on bioactivity. It appears that most biologically active particles are within $0.3 \mu \mathrm{m}$ to $10 \mu \mathrm{m}$ range [81].

(3) From animal tests, bone resorption has been observed for intra-articular UHMWPE particle injection experiments. A direct proof that UHMWPE particles induce osteolysis has been obtained through this approach. 


\section{CHAPTER 3}

\section{PROBLEM DEFinition}

\subsection{The need to prolong the life of joint-replacement components}

There are increasing numbers of total joint replacements implanted in the United States and around the world [1]. For total hip replacement, an estimated number of 170280 implant surgeries were performed in 1998 in the United States, a 5 $\%$ increase over those performed in 1994 . For total knee replacements, the number of new implant surgery are 249944 in 1998 which is a $32 \%$ increase compared to the data for 1994. These numbers indicate a rapid growth of demand for total joint replacements. Possible reasons include the increase of life expectancy, population increase, more active life style, increase of arthritis disease, and younger patients. The average life of a total hip replacement is between 10 years to 15 years and less for a total knee replacement. Once the failure of a joint implant occurs, the best solution is to perform a revision surgery by replacing the damaged parts. The clinical data show that the number of revisions for total hip replacements was 29368 in 1998, a $7 \%$ increase over the revisions reported in 1994 in United States. It also shows 21365 revisions for total knee replacements, a 34 \% increase from 1994 in the United States.

With the current lifetime of total joint replacements, the percentage of revision surgery needed for patients will increase tremendously because of younger patients 
and longer life expectancy of the population. Thus there is an urgent need to extend the life of the replacement joints.

\subsection{Potential solutions if particle induced bioactivity is understood}

It is possible to enhance the life of total joint replacement by reducing the production of the most toxic particle populations in terms of size and shape. For this purpose, one needs to know the effects of UHMWPE wear particle size and shape on bioactivity. By changing the materials properties such as crosslinking and wear durability of UHMWPE, we should be able to adjust wear of the components and control the wear particle size. By modifying the contact geometry or surface texturing the joint implant surfaces or engineering the material microstructure, one may be able to control the size and shape of wear particles. Less toxic particles generated in-vivo can prevent or delay osteolysis and bone resorption induced by wear particles.

From the viewpoint of medical treatment, if the detailed mechanism of particle induced bioactivity is understood, one can possibly discover medicines to block the pathways and inhibit the occurrence of osteolysis. One may also blend the pathwayblocking agents into the component materials to prevent resulting osteolysis. By studying the wear particles generated from joint replacements made by different manufacturers, we can possibly estimate the level of immunological response of patients. Close monitoring and medical treatment can be applied to help prevent the failure of total joint replacements. 


\subsection{Cause and effect of particle sizes and shapes}

Uncertainty exists as to how UHMWPE wear particles stimulate the sequential bioactivities and as to results in clinically observed osteolysis and bone resorption. The following questions should be addressed in order to solve this problem.

- Are all particles bioactive?

- What is the effect of particle size?

- What is the effect of particle shape?

- What is the critical threshold concentration of a particle population with certain size and shape?

In order to provide answers to the above questions, a series of issues needs to be addressed. Critical issues include (1) lack of narrowly distributed UHMWPE wear particles with controlled size and shape, (2) need of quantitative methods to describe the particle populations, and (3) a complete bioactivity study to determine critical thresholds for particle-induced biological responses. 


\subsection{Critical issues}

\subsubsection{Availability of narrowly distributed particles}

From clinical retrieval studies of wear particles, it is known that various sizes and shapes of UHMWPE particles are generated from total joint replacements [35-42]. The variety of particle sizes and shapes is an outcome from the complexity of motions and stress conditions on joints in humans' daily life. Various geometrical designs for different joints also contribute to the size and shape of particles generated in-vivo. In addition, the variety of material processing and sterilization methods from orthopedic implant manufacturers result in different UHMWPE material properties which further leads to wide spectra of UHMWPE particle sizes and shapes that are generated.

Various kinds of polyethylene particles have been prepared for biologicalresponse studies. Commercial resin powders of high-density polyethylene (HDPE) or UHMWPE are commonly used because of convenient availability [78-80, 82, 85]. HDPE resin powders have a mean particle size in the $4 \mu \mathrm{m}$ to $10 \mu \mathrm{m}$ range, and the shape of the particle is almost perfectly spherical. However, HDPE has a higher density and lower molecular weight than UHMWPE. Some studies have indicated the chemical component of particles as one critical factor for biological response. The smooth morphology of HDPE powders is also quite different from the rough particle surfaces of particles retrieved from clinical studies. Thus it is inadequate to use HDPE material for biological response experiments. Figure 3.1 shows a comparison of the particle morphology of HDPE powders, UHMWPE powders and retrieved UHMWPE particles. Many researchers applied UHMWPE resin particles. However, the mean size 
of commercial UHMWPE particles is always $40 \mu \mathrm{m}$ or larger with a broad size distribution. Only round-like UHMWPE particles are available commercially and there is lack of elongated particles. The surface energy of commercial particles may also differ from wear particles due to the difference in formation mechanism. Particles generated from a wear process tend to carry electrostatic charges. Thus the material property of the polyethylene resin powders may differ from the particles generated from a wear process. The significance of this issue cannot be ignored for linking the in-vivo or in-vitro study results to clinical situations.

To investigate the effects of size and shape of particles to biological response, narrowly distributed particles with controlled size and shape are needed. By utilizing narrowly distributed UHMWPE particles with controlled size and shape for bioactivity study, an understanding of direct biological responses influenced by particle size and shape becomes possible.

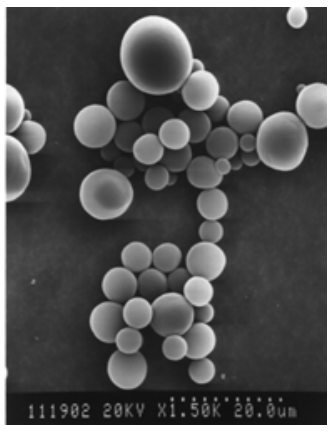

HDPE Powders

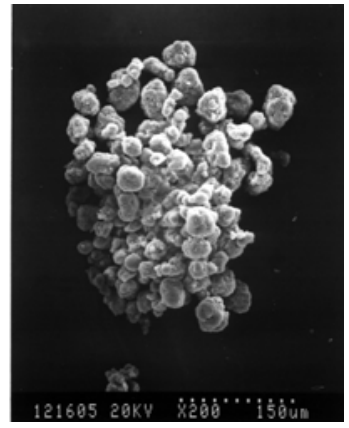

UHMWPE Powders

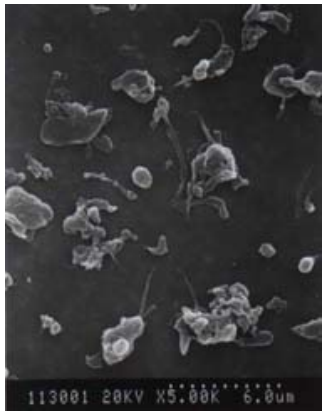

Retrieval UHMWPE Particles

Figure 3.1 Comparison of polyethylene powders and particles. 


\subsubsection{Characterization of particles}

The morphology of wear particles is strongly related to the surface properties of material and wear mechanism under a set of operating conditions. According to various features of wear particles, possible wear mechanisms and failures of the system may be identified. It is feasible to judge the wear particles objectively by visual inspection as belonging to different populations. However, quantitative and systematic methods for determining the morphological attributes in terms of their size, shape, and surface texture are desirable to understand their effects on bioactivity.

\subsubsection{Bioactivity tests of UHMWPE particles}

Various in-vitro cell-culture studies and in-vivo animal tests of UHMWPE particles have been used to evaluate biological response induced by particles. The advantages of cell-culture studies are control of cell numbers, cell-incubation environment, in-situ observation of particles-cells reactions, and easy measurement of cell-released products. However, the complexity of the biological environment is lacking due to the simplification of the studies. In contrast, animal tests simulate a testing environment more similar to the real situation. However, the disadvantages include the variation between test animals, difficult control of testing parameters (such as cell numbers, temperature), and increase of uncertainty factors.

Before applying various particle sizes and shapes for bioactivity tests, proper experimental design is required to understand the particle-induced osteolysis in total joint replacements. 


\subsection{Approaches}

\subsubsection{Generation of particles by surface texturing}

In order to generate sufficient narrowly distributed UHMWPE particles with controlled size and shape, we proposed to rub UHMWPE against a textured surface that contains numerous cutting features. The surface textures should be designed so that the individual feature on the surface is acting as a cutting device to UHMWPE material. Geometric constraints provided by the cutting edges on the surface texture define the volume of a particle. By changing the geometry of the surface textured cutting features and the mechanical conditions of the micro-cutting process, the particle size and shape can be changed. Thus by designing a geometrically patterned surface texture, particles of similar size and shape can be generated.

\subsubsection{Modeling of particle-generation process}

It is desirable to develop a model to predict the size and shape of particles generated with given surface texture dimensions and mechanical conditions of the particle-generation process. The mechanism of particle generation should be investigated by focusing on the particle generation by a cutting feature acting on UHMWPE. One approach is to perform the simple model experiments to simulate a micro-cutting process by observing the cutting process with the use of scaled-up cutting edges. In-situ observation of the cutting process is feasible by microscopic video- and force measurement systems. By changing the geometry of cutting edges and mechanical conditions of the cutting process, the critical criteria of particle 
generation can be obtained. With a given cutting-edge geometry and mechanical conditions, a contact mechanics approach may be used to calculate the plastic deformation of UHWMPE material. The model should be able to calculate the surfacetexture dimensions and mechanical conditions required for generation of UHMWPE particles with certain size and shape.

\subsubsection{Characterization of particles}

In order to quantify the effects of UHMWPE particles on induced bioactivity, a biophysical approach is proposed to analyze phagocytosis of particles by macrophage cells. By integrating the size and shape information of the particles, we hope to derive parameters that correlate with the level of bioactivity induced by a particle population. By doing so, we can further identify the critical particle size and shape for bioactivity.

\subsubsection{Bioactivity tests of UHMWPE particles}

Bioactivity tests of UHMWPE particles have been performed in collaboration with medical laboratories. During the development of our particle-generation procedure, the particles were regularly examined at Wayne State University for possible biological contamination. Furthermore, an air pouch test performed at Wayne State University was adopted as the standard method to test UHMWPE particles with various sizes and shapes. The air pouch model of inflammation, adopted here, resembles the peri-prosthetic tissue encountered in aseptically loosened prosthetic components to evaluate the cellular response to UHMWPE particles in vivo. 


\section{CHAPTER 4}

\section{Procedures for GeNERATION AND Collection of PARTicles}

\subsection{Introduction}

The generation of UHMWPE particles by a wear process using surface that has been textured is introduced in this chapter. The goal of the particle-generation process is to produce sufficient amounts of UHMWPE particles with controlled size and shape. A procedure for the generation of such particles by a wear process is developed that allows precise measurement of the amounts of wear particles, eliminates possible particle contamination, and maintains a sterilized environment. The procedures for collecting the UHMWPE particles are described. These particles are needed for particle characterization and subsequent bioactivity testing. A high particle recovery rate is desirable so that the results of particle analysis can be representative and the production rate of particles can be increased. The sizes and shapes of the particles were characterized by a computer imaging analysis. The chemical composition of the particles was also verified by FTIR measurements. By adjusting the $\mathrm{pH}$ value of the washing solution, we found that the particle recovery rate can be raised to above $90 \%$. The detailed operation procedures are described in the sections below. 


\subsection{Generation of UHMWPE particles}

\subsubsection{Literature review of UHMWPE particle generation}

There have been several methods applied to generate UHMWPE particles invitro. To simulate the wear process, pin-on-disk unidirectional wear tests have been used to generate UHMWPE particles [90-93]. For that purpose an UHMWPE pin was applied to slide against a stainless steel flat surface. By changing the roughness of the steel surfaces, different average particle sizes were generated. To obtain more narrowly distributed particles, Green et al. [81] further divided the particles into different size ranges by filtration. Mean particle sizes of $0.21 \mu \mathrm{m}, 0.49 \mu \mathrm{m}, 4.3 \mu \mathrm{m}$, and 7.2 $\mu \mathrm{m}$ were prepared. The particles obtained from the wear process with a randomly polished steel surface all have a spherical-like shape.

Shanhbag et al. [94] applied attrition of commercial UHMWPE particles under cryogenic conditions. The larger resin particles were filtered and the finer UHWMPE particles were recovered with a low yield rate $(2 \mathrm{~g}$ of fine particles were harvested from $480 \mathrm{~g}$ starting material). The particle population contained fibrils and sphericallike particles. The particle size ranged from $0.1 \mu \mathrm{m}$ to $33 \mu \mathrm{m}$ in diameter, with a mean of $2.3 \mu \mathrm{m}$. It was difficult to isolate the particles with similar shapes in a particle population. Although sizes and shapes of particles similar to the clinically retrieved particles were claimed, the uniformity and control of sizes and shapes of a particle population was not achieved.

Yarovoy et al. [95] fabricated micrometer and sub-micrometer sized UHMWPE particles from an organic solution by employing a precipitation method. 
This method involves the formation of an emulsion in which droplets of a solution are dispersed in a non-solvent medium. Since the emulsions are very sensitive to changes in temperature, the concentration and ratio between the components, the size of the droplets, and the size of the precipitated particles can be controlled. However, only spherical-like particles were produced. The variation of the synthesis process of UHMWPE may result in differences of the physical properties from the UHMWPE for clinical use. This method is also inadequate to produce particles for systematically investigating the effects of UHMWPE particle size and shape on bioactivity systematically.

\subsubsection{Concepts of surface texturing}

We use a micro-cutting principle to generate wear particles. Surface texture patterns act as cutting edges against UHMWPE under rubbing conditions. Geometric constraints provided by the texture topography and the relative sliding determine the volume for the generated particles. Thus by designing a repetitive geometric pattern, particles of similar size and shape can indeed be generated. Dimensions of the cutting edges play a significant role in a micro-cutting process. Curvature or radius of the cutting edge is a key factor to control the local asperity contact stress. The 3dimensional spatial arrangement of the cutting edges controls the geometric constraints and results in the formation of different particles. Thus, different kinds of wear particles can be generated by micro-cutting processes with different surfacetexture designs. 


\subsubsection{Preparation of materials}

GUR 415 UHMWPE $^{1}$ cylinder pins were machined to the dimensions of 6.35 $\mathrm{mm}$ in diameter and $25.4 \mathrm{~mm}$ in length with diamond turning on both end surfaces. No further polishing on the material is allowed to avoid residual stresses remaining on the material surface. UHMWPE is hydroscopic, i.e. it absorbs water. The content of water absorbed will change the unit weight per volume and also change the mechanical property. During the wear tests, one end of the UHMWPE pin is fixed by a sample holder and the other end is immersed in the water. In order to maintain consistency of the test, the UHMWPE pin must be water saturated at the beginning of the test. It can prevent the pin from further absorbing water during the wear test. During the wear tests, the UHMWPE pin is tested in a closed space wrapped by a plastic membrane. The water-vapor pressure reaches equilibrium and the water content in the UHMWPE pin remains the same.

To determine the time needed to reach saturation with water, six pins were immersed in the water and they were weighed periodically as shown in Figure 4.1. We have concluded that the UHMWPE must be pre-soaked in water at least 15 days before testing to reach water-saturation equilibrium.

${ }^{1}$ GUR 415 is a brand name of UHMWPE resin produced by Hoechese-Celanese.

Certain commercial equipment, instruments, or materials are identified in this paper to specify adequately the experimental procedure. Such identification does not imply recommendation or endorsement by the National Institute of Standards and Technology, nor does it imply that the materials or equipment identified are necessarily the best available for the purpose. 
P1

Initial weight $=743.25 \mathrm{mg}$

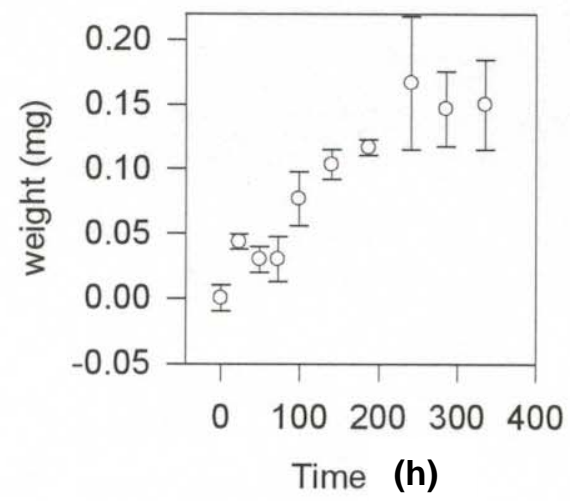

P3

Initial weight $=741.42 \mathrm{mg}$

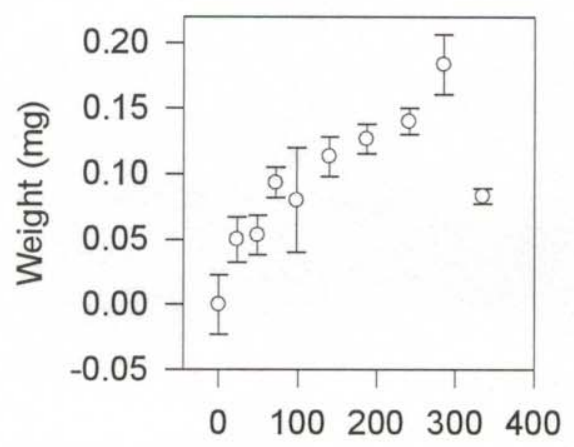

Time (h)

P5

Initial weight $=746.34 \mathrm{mg}$

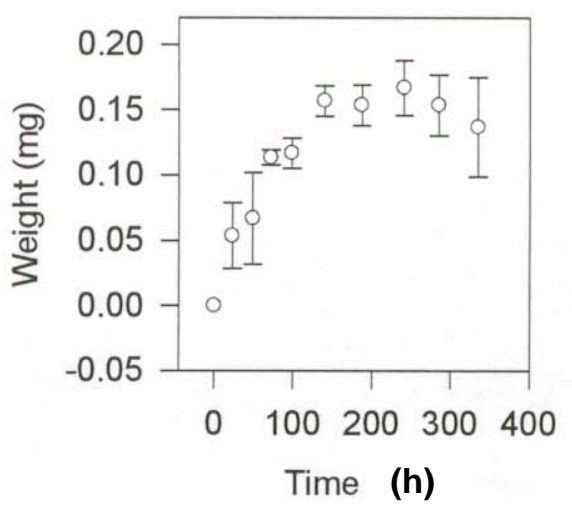

P2

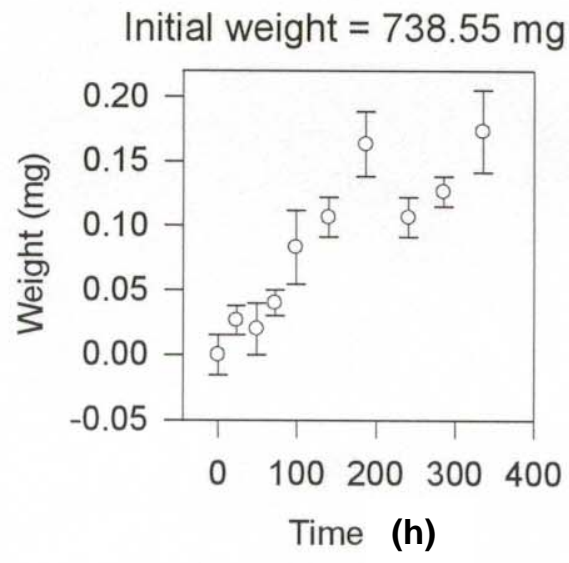

P4

Initial weight $=750.38 \mathrm{mg}$

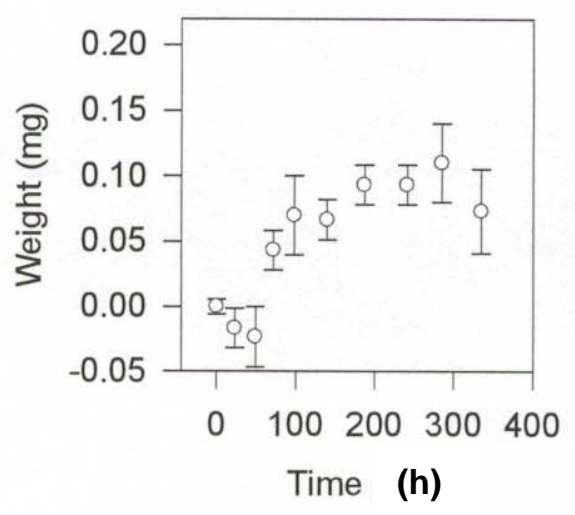

P6

Initial weight $=739.72 \mathrm{mg}$

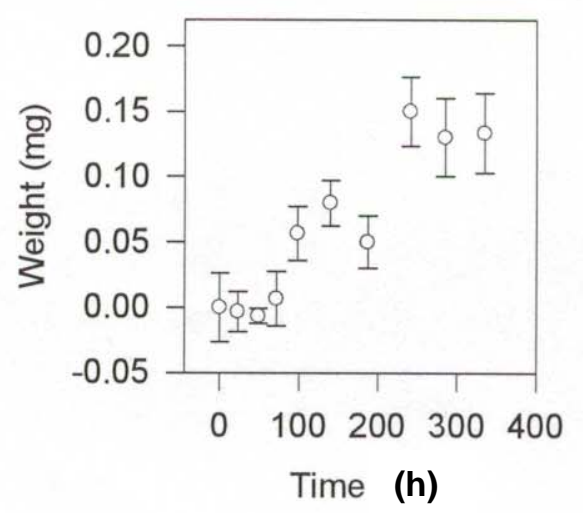

Figure 4.1 Weight change of pre-soaked UHMWPE pins. 
To avoid chemical, particle, and biological contamination in the process for particle generation, all materials and liquids were purified and sterilized. The following procedure was carried out in a clean room and the operator always wore a hat, mask, and powder free gloves for every step of the operation.

The water used in this study was purified through deionization, filtration by 0.1 $\mu \mathrm{m}$ pore-size membrane and double distillation to avoid both chemical and particle contamination. Then the water went through a high-temperature, high-pressure autoclave process to eliminate possible microbial contamination. Prior to the test, all parts involved in the experiments were immersed in a $70 \%$ ethanol $+30 \%$ purified water solution (volume fraction) overnight for sterilization. The ethanol also went through filtration by $0.1 \mu \mathrm{m}$ pore-size membrane to avoid particle contamination.

Before the tests, containers, sample holders, and textured plate, made of 316 stainless steel, must be cleaned by acetone and hexane solution (volume ratio 1:1) in an ultrasonic bath for $3 \mathrm{~min}$. Then they were cleaned with a detergent in an ultrasonic bath for another $3 \mathrm{~min}$. The parts were then rinsed in the purified water and blowed dry by filtered compressed nitrogen gas.

\subsubsection{Linear reciprocating wear process}

The linear reciprocating wear test method was used to generate UHMWPE wear particles. ASTM F732 is used as a guideline. The setup of the facility is shown in Figure 4.2. With the driving mechanism of the linear reciprocating wear tester, a constant rotation speed is kept while the test is performed. Thus the velocity of the 
linear reciprocating motion is a sinusoidal distribution depending on the stroke length and phase angle of the drive mechanism.
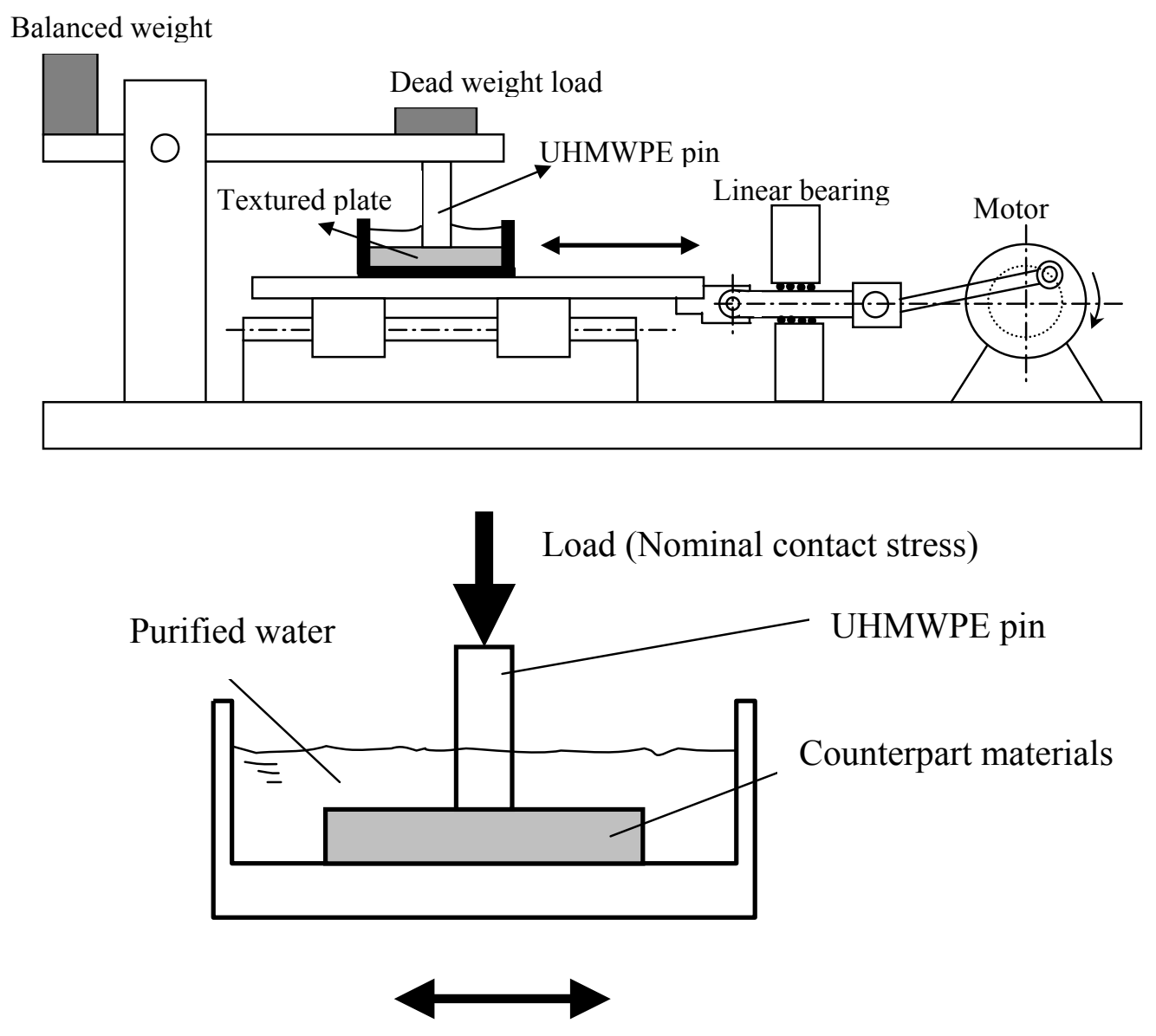

Reciprocating sliding

Figure 4.2 Schematic representation of linear reciprocating wear tester. 
Before tests of particle generation, one UHMWPE pin and three control pins were dried by blowing with filtered compressed nitrogen and weighed three times. Three control pins were soaked in water at the same water level as the wear-testing condition. The sample holders and the textured plate were taken from the (alcohol/purified water) solution and were immediately blowed dry by filtered compressed nitrogen. The UHMWPE pin was then mounted on the sample holder and the textured plate was fixed at the lower container. Ultrajet clean air was applied to the UHMWPE pin and the lower container and then purified water was added to the container. Then the UHMWPE pin with its holder and the lower container were wrapped by a clean plastic membrane. The contact surface between UHMWPE pin and textured plate was leveled and the rotation speed of the motor was checked before the normal load was applied. Then a dead weight was applied above the UHMWPE pin holder and the test was started. The rotation speed was monitored during the wear process. The linear reciprocating motion stopped after a specified duration.

\subsection{Collection of UHMWPE particles}

\subsubsection{Particle-collection procedure}

A flow chart of the particle-collection process is shown in Figure 4.3. After the wear tests, the UHMWPE wear particles suspended in the water were poured into a sterilized glass beaker. Purified water with adjusted $\mathrm{pH}$ value was prepared in a Guth bottle that has the capability to inject water with a constant velocity. The sample, sample holder, and parts that came into contact with particles were repeatedly rinsed 
in a sterilized glass beaker by ejecting purified water from the Guth bottle. The particles suspended in the water went through a filtration process. To measure the weight of the collected UHMWPE wear particles, a polycarbonate filter membrane with $0.1 \mu \mathrm{m}$ pore size (Milipore) was put into a plastic dish with the cap. The weight was measured with a resolution of $\pm 0.01 \mathrm{mg}$. The filter membrane was then placed on the filter holder of a vacuum filtration system. Particles with water in the beaker were then poured into the filtration system and again the Guth bottle with purified water was used to wash the beaker and the upper filter holder until no particles were observed on the cylinder wall. After the filtration process, the UHMWPE wear particles were collected on the surface of the filter membrane. The filter membrane was then put back in the plastic dish in the clean room for three hours to evaporate the water absorbed by the membrane. The weight of the filter membrane with particles in the plastic dish with the cap was then measured and the weight of the collected wear particles was obtained from the difference of the two weight measurements. 


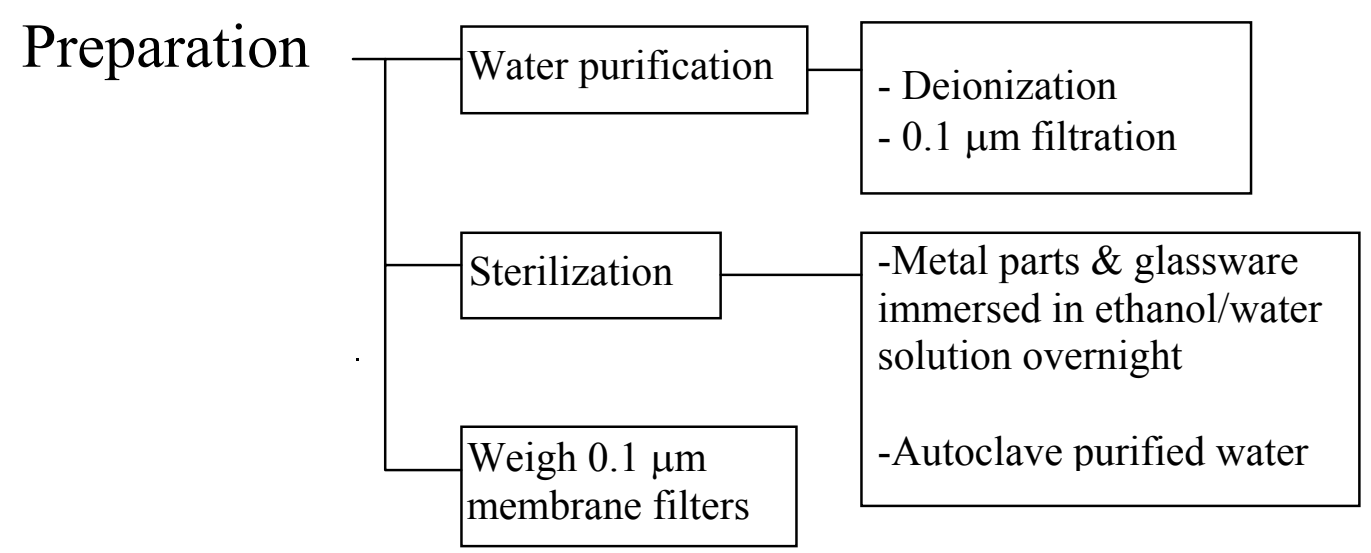

\section{Collection}

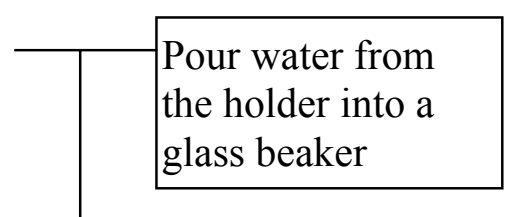

\begin{tabular}{|l|}
\hline $\begin{array}{l}\text { Wash sample holder } \\
\text { with } \mathrm{pH} 5.5 \text { water }\end{array}$ \\
$\begin{array}{l}\text { Vacuum filtration } \\
(0.1 \mu \mathrm{m} \text { membrane })\end{array}$ \\
Dry the filter(s) in air
\end{tabular}

Measurement

Weigh the filter(s) with particles

Figure 4.3 Particle-collection procedure. 


\subsubsection{Effect of $\mathrm{pH}$ value on particle recovery}

It turned out that UHMWPE wear particles apparently carry a weak electrostatic charge and have a tendency to stick to the glass beaker surface during the particle-collection process. By adjusting the $\mathrm{pH}$ value of the water, the charges on the particles can be controlled for a fuller recovery rate. A simple experiment was performed to find the optimum $\mathrm{pH}$ value for particle-removal process from glass. A known amount of UHMWPE particles was dispersed in filtered isopropyl alcohol in the ultrasonic bath for $3 \mathrm{~min} .1 \mathrm{~mL}$ of the UHMWPE isopropyl alcohol suspension was dropped on a Pyrex glass slide. The number of particles in a given area was counted under an optical microscope. Hydrochloric acid solution was added to purified water (deionized, $0.1 \mu \mathrm{m}$ pore-size filtered, and double distilled) to prepare washing solutions with different $\mathrm{pH}$ values. $1 \mathrm{~mL}$ of the washing solutions was used to rinse the glass slide with particles on it, and this procedure was repeated 5 times. Then the particles remaining on the glass slides were counted again under optical microscope. The number of particles that were removed by the rinsing process was obtained and the degree of particle removal can be calculated ( 0 for no particle removal, and 1 for all particles removal). Figure 4.4 shows that washing water with a $\mathrm{pH}$ value around 5 to 6 has the best ability to remove the UHMWPE particles from the glass slides. Several particle generation wear procedures with different total wear loss were performed. A comparison of particle recovery rate between the cases of particle collection using $\mathrm{pH} \approx 5.5$ washing water and the cases of particle collection using no $\mathrm{pH}$ control is presented in Figure 4.5. It indicates that by adjusting the $\mathrm{pH}$ value of 
washing water to 5.5 , the particle recovery rate was improved from $40 \%$ to $60 \%$ to nearly $90 \%$.

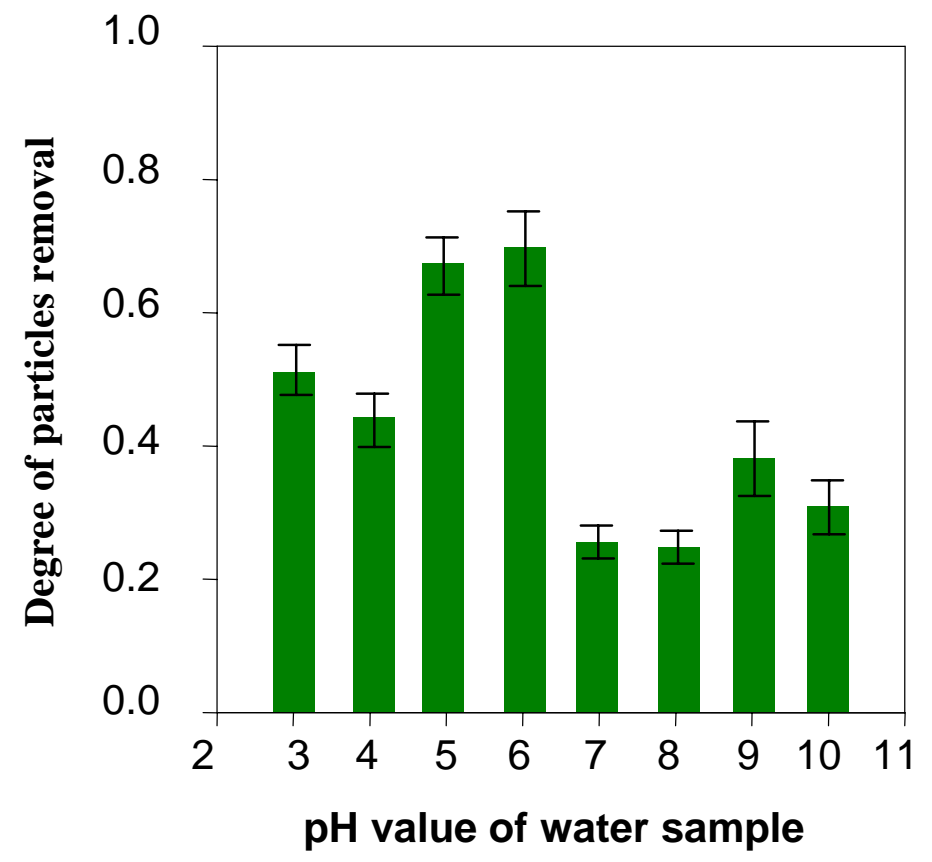

Figure 4.4 Effect of $\mathrm{pH}$ value of water on removing particles on the glass slide. 


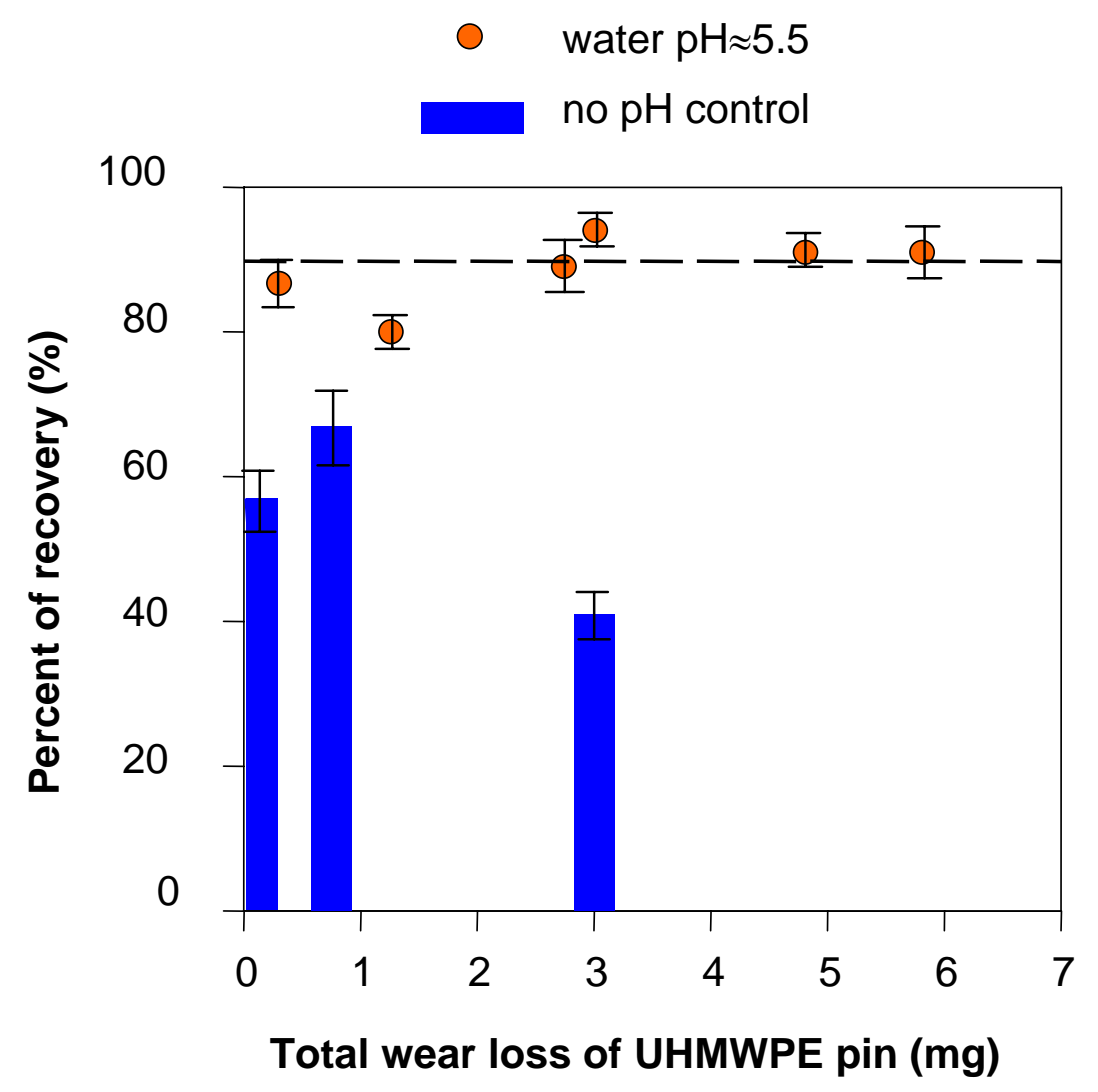

Figure 4.5 Effect of $\mathrm{pH}$ value of water on particle-recovery rate. 


\subsection{Particle characterization}

\subsubsection{Chemical composition of particles}

To detect the chemical compositions of the collected wear particles, the microscopic Fourier transform infrared spectroscopy (FTIR) technique was applied. The collected particles were mixed with $\mathrm{KBr}$ powders to form a sample. This sample was then analyzed by FTIR in a transmission mode. Bulk UHMWPE raw material was also examined by using attenuated total-reflection Fourier-transform infrared spectroscopy (ATR-FTIR) in a contact mode. The IR absorbance spectra are compared in Figure 4.6. The peaks in the spectra represent the methyl groups $(\mathrm{C}-\mathrm{H}$ stretching in (2800-3100) $\mathrm{cm}^{-1}, \mathrm{CH}_{2}$ scissors near $1450 \mathrm{~cm}^{-1}$, and $\mathrm{CH}_{2}$ rocking at $\left.730 \mathrm{~cm}^{-1}\right)$ that are the characteristic absorption for polyethylene. The noise seen from the spectrum of collected particles is due to small quantity of particles. The uncertainty of the measurement comes from the noise of the signal. A match of the IR spectrum of collected particles and the bulk UHMWPE material verifies the main chemical composition of collected wear particles is polyethylene.

\subsubsection{Image analysis of particles}

The polycarbonate membrane filters with wear particles were coated with a thin layer of gold. Then SEM observation of the particles on the filter was performed. The magnification was adjusted to clearly detect the boundaries of the particles. At least 200 particles were analyzed in each experiment. The photographs were scanned into computer image files. The Scion Image program for Windows 95 was applied to 
analyze the particles. Different parameters for particle size and shape can be calculated by incorporating an appropriate subroutine in the program. In most cases throughout this study, the longer axis of the particle and the minor axis of the particle represent the particle length and width. The aspect ratio is defined as the ratio of particle length to particle width. The results of the image analysis will be given for the particlegeneration processes in the following chapters.

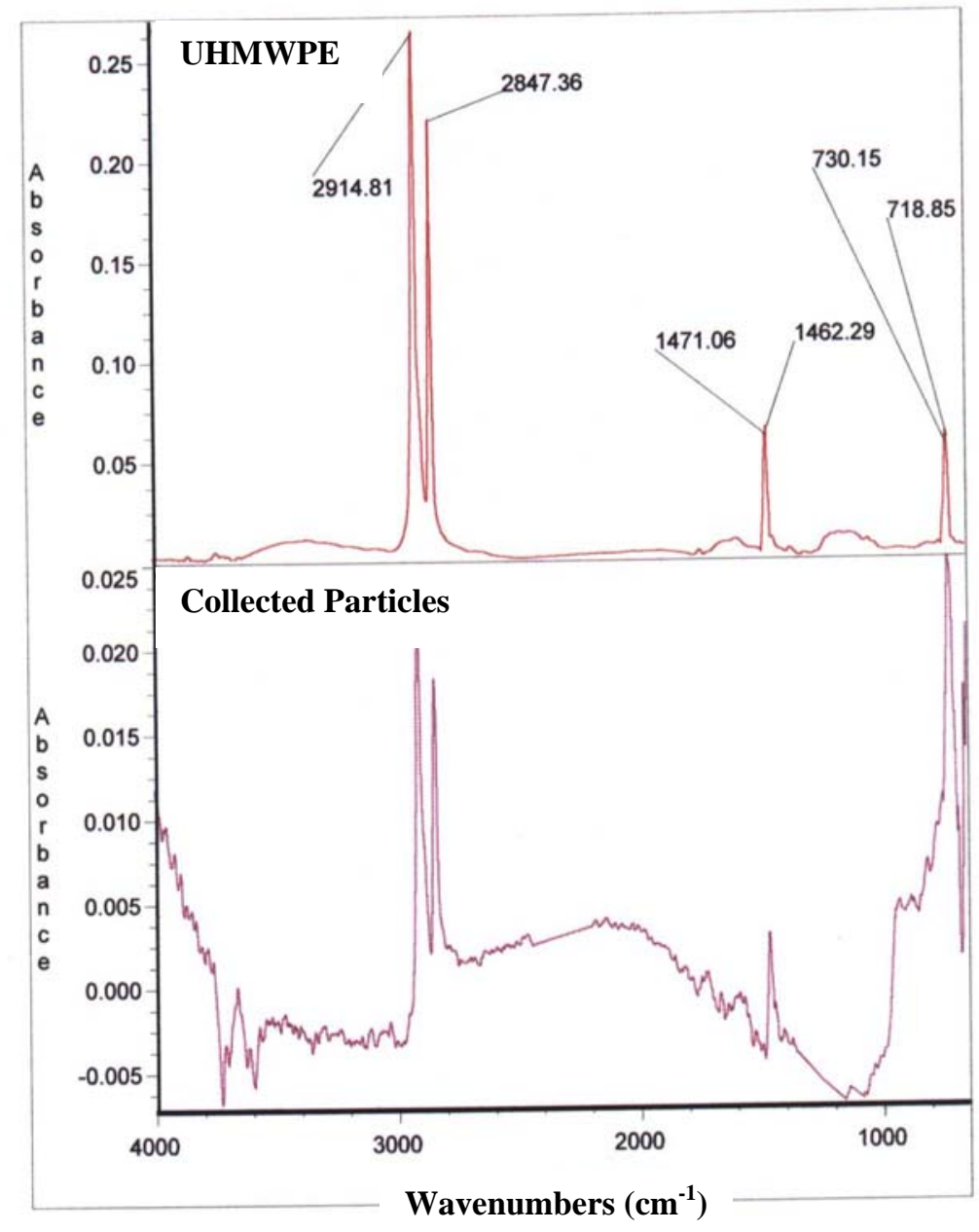

Figure 4.6 IR absorbance spectra of UHMWPE bulk material and of collected particles. 


\section{CHAPTER 5}

\section{PARTICle GeNERATION WITH SCRATCHED STEEL SURFACES}

\subsection{Introduction}

This chapter describes the first attempt at surface texturing to prove feasibility of concept. Stainless steel plates were roughened by $\mathrm{SiC}$ abrasive papers in order to generate surface textures. Then UHMWPE pins were rubbed against them to generate particles. Unidirectional and crosshatched patterns were made with $\mathrm{SiC}$ abrasive papers. Textured surfaces with different roughness were prepared by using different grades of abrasive papers. The scratched stainless-steel surfaces were then mounted on the wear test facility to generate UHMWPE particles. Effects of the feature geometry and roughness of the textured surfaces are discussed. To further improve the uniformity of textured surfaces, single diamond tips were utilized to scratch the stainless steel surface with specified penetration depth and length. The effect of uniformity of the surfaces on the particle distribution is shown in this chapter.

\subsection{Particle generation with abrasive-paper made surfaces}

\subsubsection{Preparation of steel surfaces with SiC abrasive papers}

Stainless steel 316 plates $(38 \mathrm{~mm}$ in diameter and $6.35 \mathrm{~mm}$ in thickness) were polished to a surface roughness $(\mathrm{Ra})$ of less than $0.025 \mu \mathrm{m}$ (Polishing progression with the sandpapers: 120grit $\rightarrow 320$ grit $\rightarrow 400$ grit, and then diamond suspensions: $6 \mu \mathrm{m}$ 
$\rightarrow 1 \mu \mathrm{m})$. Texturing of the steel plate was achieved by making systematic onedirectional grooving passes using silicon carbide ( $\mathrm{SiC})$ abrasive papers made by Teco. The grooving passes were made under a nominal contact pressure of $2 \mathrm{kPa}$. Each pass was $150 \mathrm{~mm}$ long. The surface was then returned to the starting position and scratched again. The procedure is shown schematically in Figure 5.1. For unidirectional textures, twenty passes were made. To produce a crosshatched pattern, the steel plate was rotated $90^{\circ}$ after each pass for a total of twenty passes. A single grit size of $\mathrm{SiC}$ abrasive paper was used to texture each stainless-steel plate. The textured plate was then cleaned ultrasonically in solvents and detergent, followed by rinsing with purified water and drying with nitrogen. Examples of SEM photos of unidirectional and crosshatched surface textures are shown in Figure 5.2.

The steel surface made with abrasive papers was then mounted on the linear reciprocating wear machine. The particle generation process proceeded and the particles were collected and analyzed as described in Chapter 4.

\subsubsection{Results}

The wear experiments were conducted under the following conditions: normal load is $196 \mathrm{~N}$ (nominal contact pressure $\approx 6 \mathrm{MPa}$ ), stroke length is $19.05 \mathrm{~mm}$, frequency is $1.5 \mathrm{~Hz}$; average sliding velocity is $57.2 \mathrm{~mm} / \mathrm{s}$, duration is $2 \mathrm{~h}$. Purified water was used as lubricant. Table 5.1 shows the roughness of the steel plates, wear loss, particle length and width, and aspect ratio from four different surface textures 
including unidirectional and cross-hatched surface texture features made by 120 grit and 320 grit $\mathrm{SiC}$ abrasive papers.

Top View

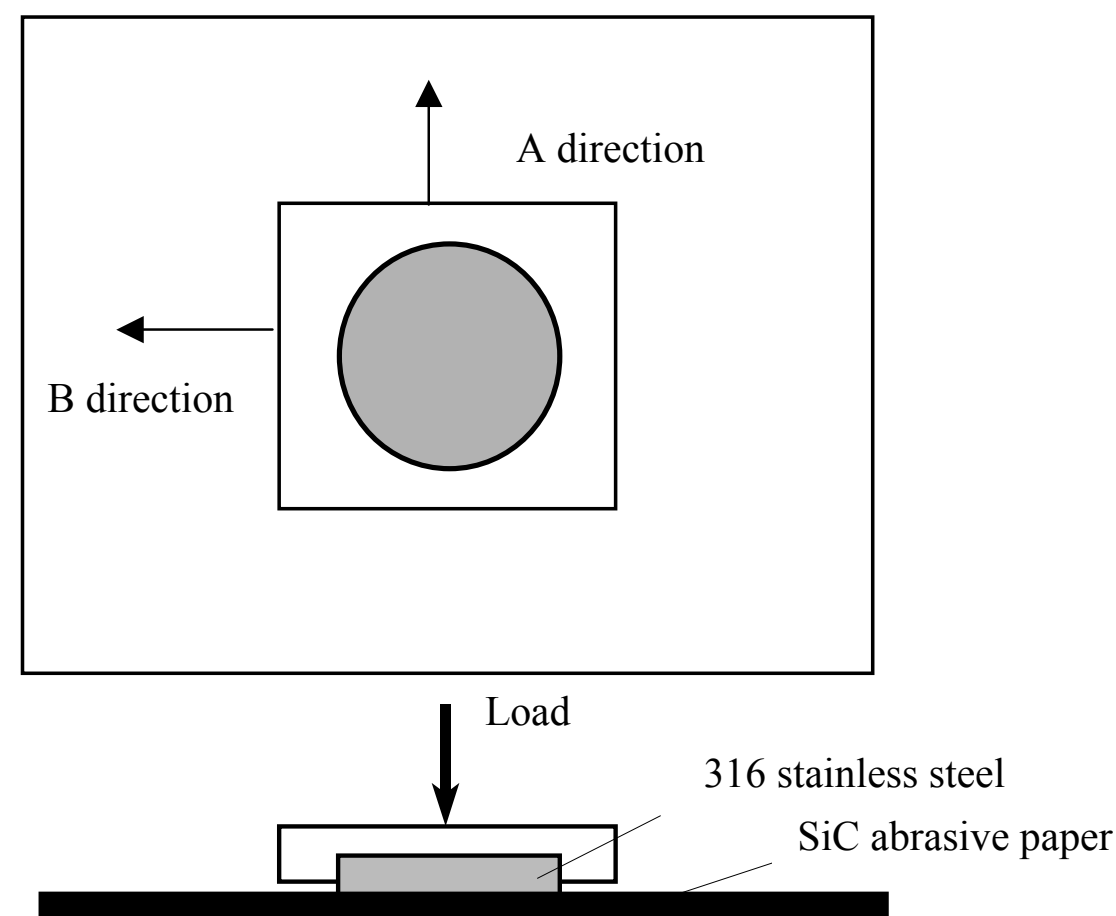

PROCEDURES:

Unidirectional textures:

1. Scratch along A direction (150mm long)

2. Use a new paper and repeat step 1

3. Repeat steps $1 \& 2$ for 20 times

Cross-hatched textures:

1. Scratch along A then B directions (150mm long)

2. Use a new paper and repeat step 1

3. Repeat steps $1 \& 2$ for 10 times

Figure 5.1 Procedure of surface texturing with $\mathrm{SiC}$ abrasive papers. 


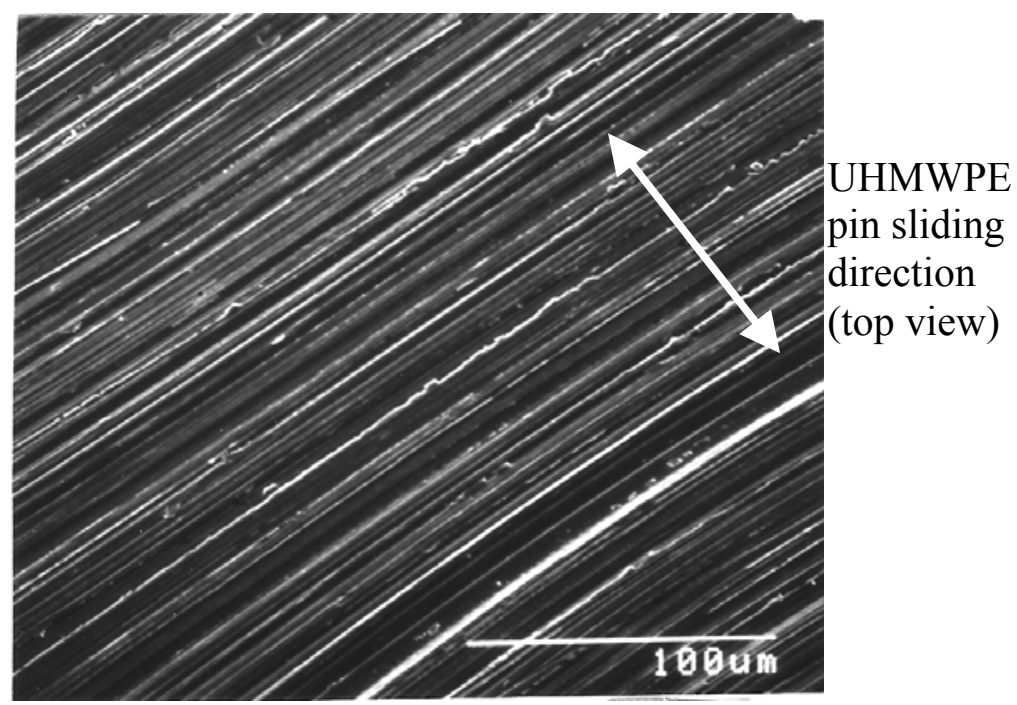

(a)

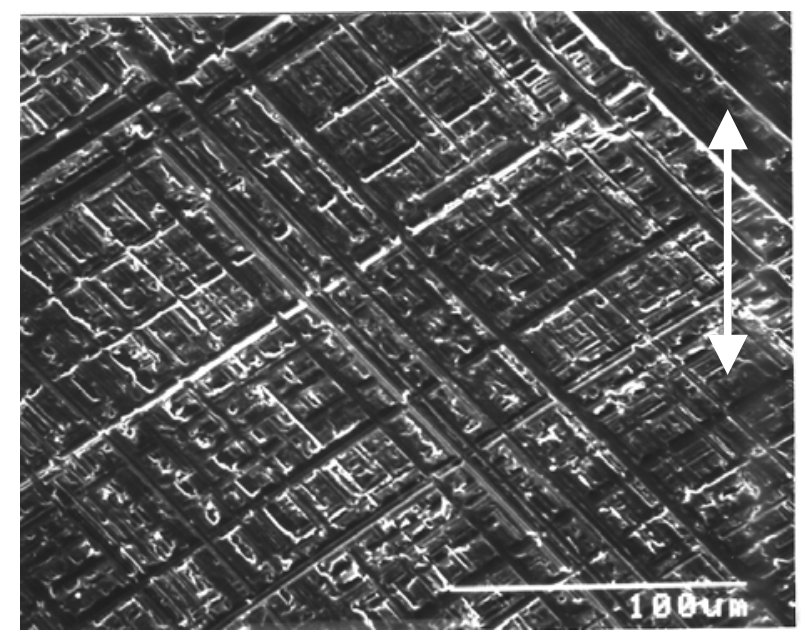

UHMWPE pin sliding direction

(top view)

(b)

Figure 5.2 (a) Unidirectional and (b) cross-hatched surface textures made on 316 stainless steel plate. 
Table 5.1 Results of particle generation with abrasive paper made surfaces.

\begin{tabular}{|l|l|l|l|l|}
\hline & 120 grit, // & 120 grit, \# & 320 grit, // & 320 grit, \# \\
\hline Wear $(\mathrm{mg})$ & $4.81 \pm 0.08$ & $2.66 \pm 0.03$ & $1.26 \pm 0.04$ & $0.30 \pm 0.06$ \\
\hline Mean particle length $(\mu \mathrm{m})$ & $47.0 \pm 19.0$ & $22.7 \pm 10.6$ & $31.6 \pm 16.7$ & $12.4 \pm 7.0$ \\
\hline Mean particle width $(\mu \mathrm{m})$ & $14.8 \pm 3.2$ & $12.7 \pm 3.0$ & $10.1 \pm 2.8$ & $6.4 \pm 2.4$ \\
\hline Mean particle aspect ratio & $3.44 \pm 1.7$ & $1.89 \pm 0.7$ & $3.60 \pm 1.9$ & $2.07 \pm 0.8$ \\
\hline
\end{tabular}

Note: //: unidirectional texture; \#: crosshatched texture. (mean \pm standard deviation)

Wear was calculated based on the mass difference of the UHMWPE pin before and after the test. It has been shown in Chapter 4 that more than $90 \%$ of the generated particles were recovered. The SEM micrographs, distributions of length and aspect ratio of the particles generated with the four different surfaces are shown in Figures 5.3-5.6. The mean particle dimension \pm standard deviation are also shown in the figures. Generally speaking, the particles generated from the wear process have irregular and rough edges similar to clinically retrieved particles. The different particle shapes generated by the unidirectional and crosshatched surfaces are shown in the figures. The mean particle size is controlled by the roughness of the surface. Effects of the surface roughness and surface texture styles will be discussed in detail in the following sections. 
(a)

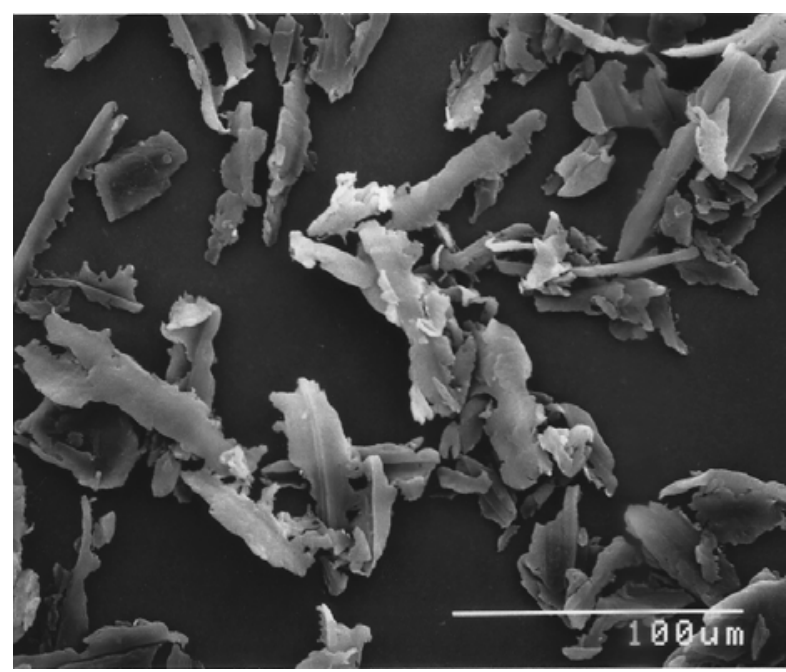

(b)

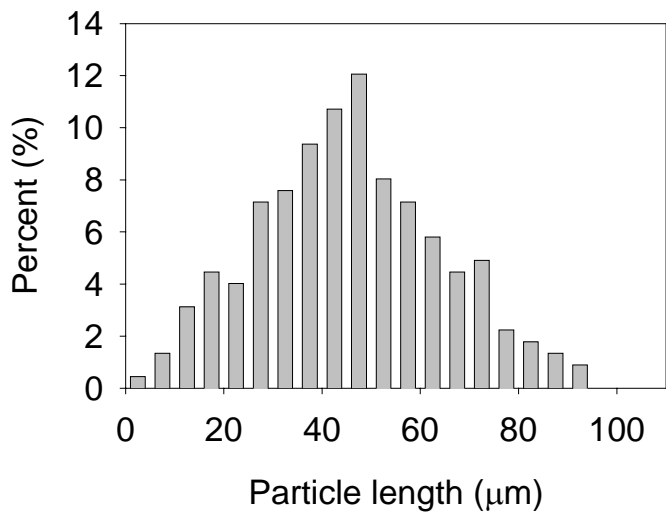

Mean particle length

$=(47.0 \pm 19.0) \mu \mathrm{m}$

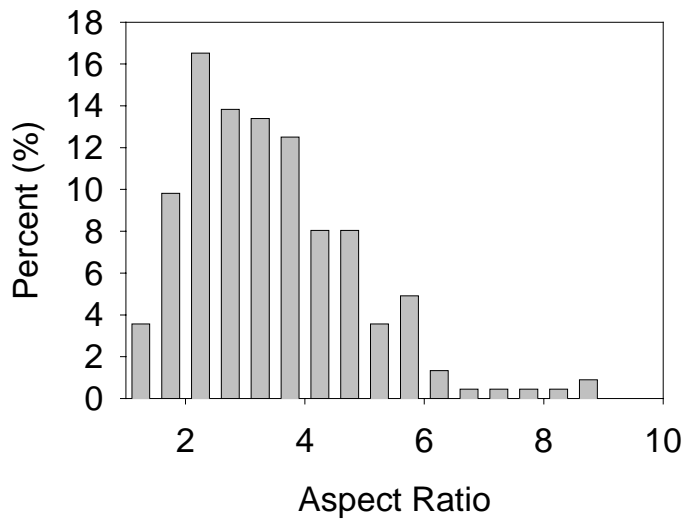

Mean aspect ratio

$=3.44 \pm 1.7$

Figure 5.3 Particles generated with 120 grit abrasive paper made unidirectional stainless steel surface. (a) SEM micrograph, (b) Distribution of particle length, (c) Distribution of particle aspect ratio. 
(a)

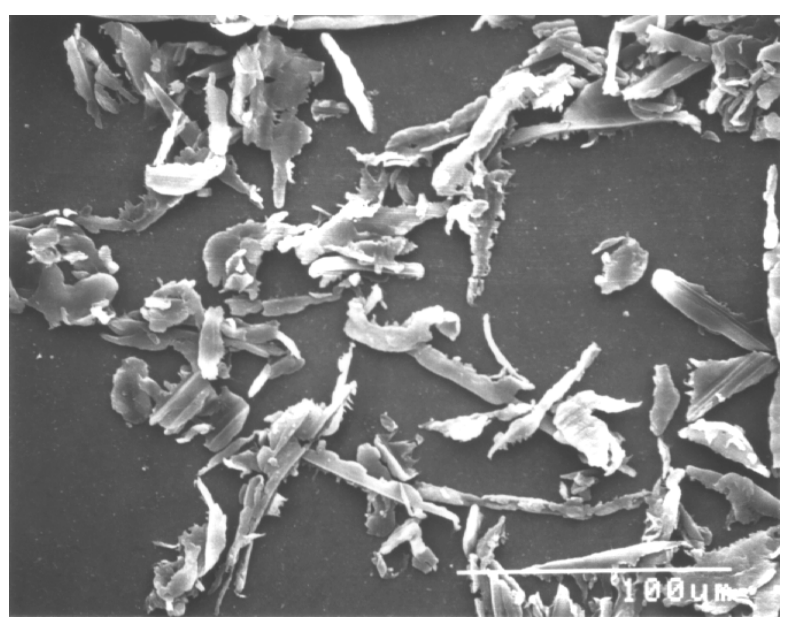

(b)

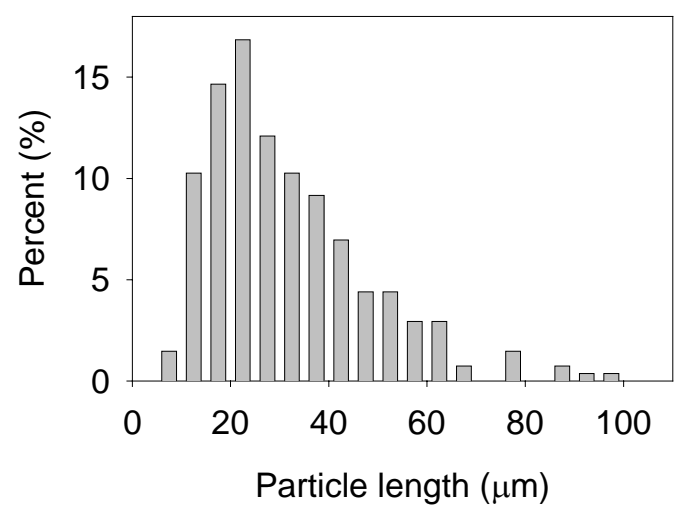

Mean particle length

$=(31.6 \pm 16.7) \mu \mathrm{m}$

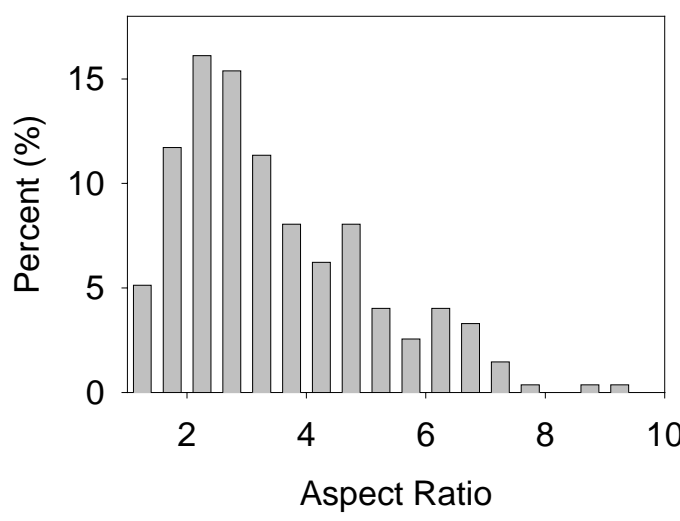

Mean aspect ratio

$=3.6 \pm 1.9$

Figure 5.4 Particles generated with 320 grit abrasive paper made unidirectional stainless steel surface. (a) SEM micrograph, (b) Distribution of particle length, (c) Distribution of particle aspect ratio. 
(a)

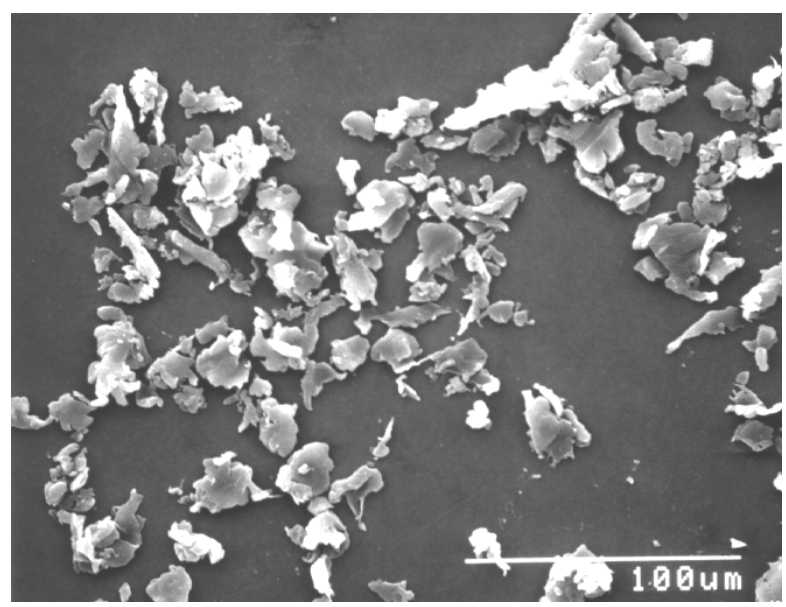

(b)

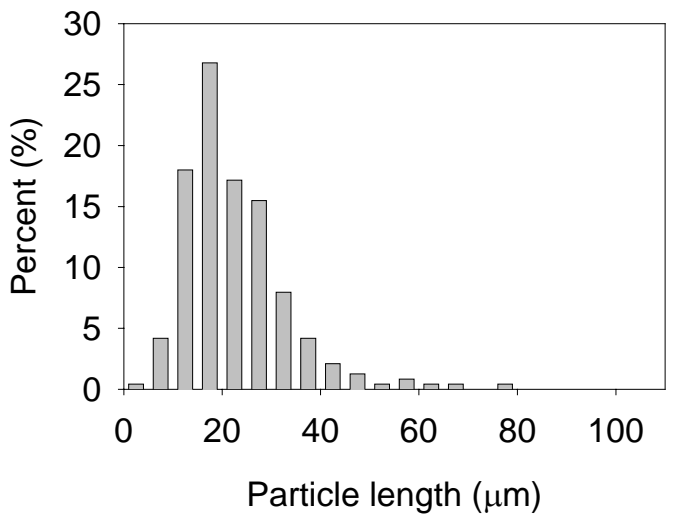

Mean particle length

$=22.7 \pm 10.6 \mu \mathrm{m}$

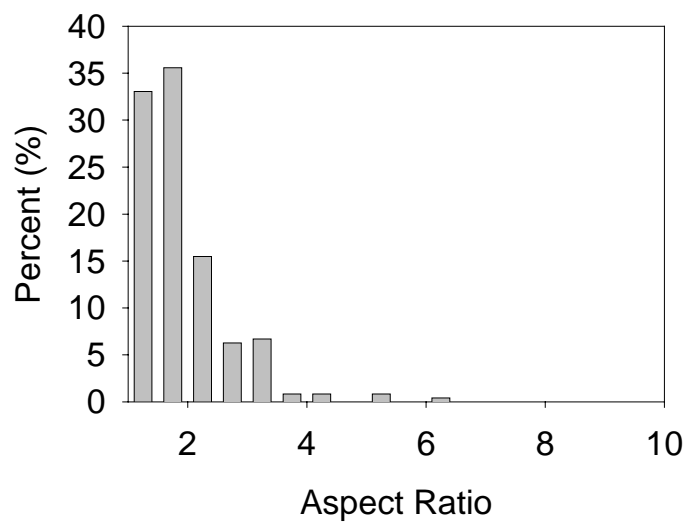

Mean aspect ratio

$=1.89 \pm 0.7$

Figure 5.5 Particles generated with 120 grit abrasive paper made crosshatched stainless steel surface. (a) SEM micrograph, (b) Distribution of particle length, (c) Distribution of particle aspect ratio. 
(a)

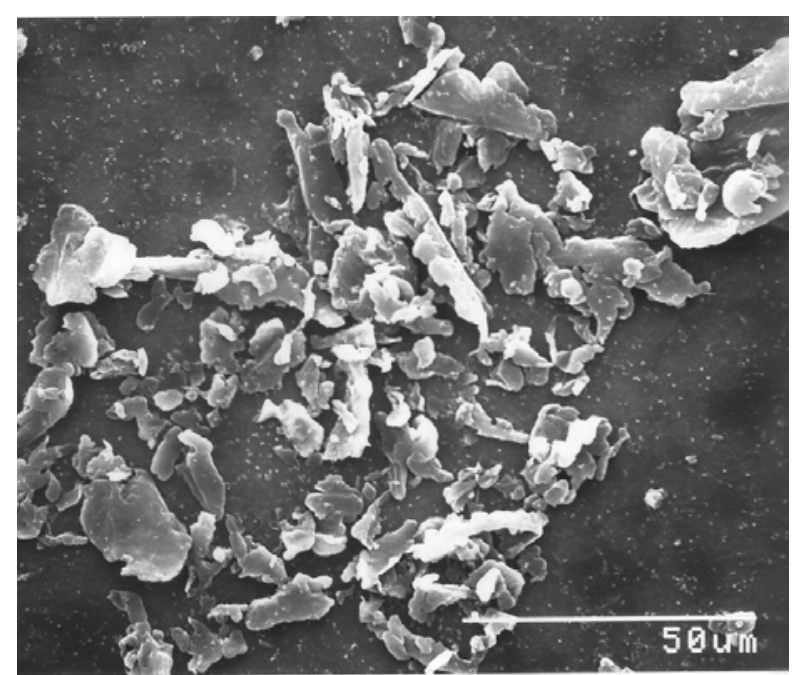

(b)

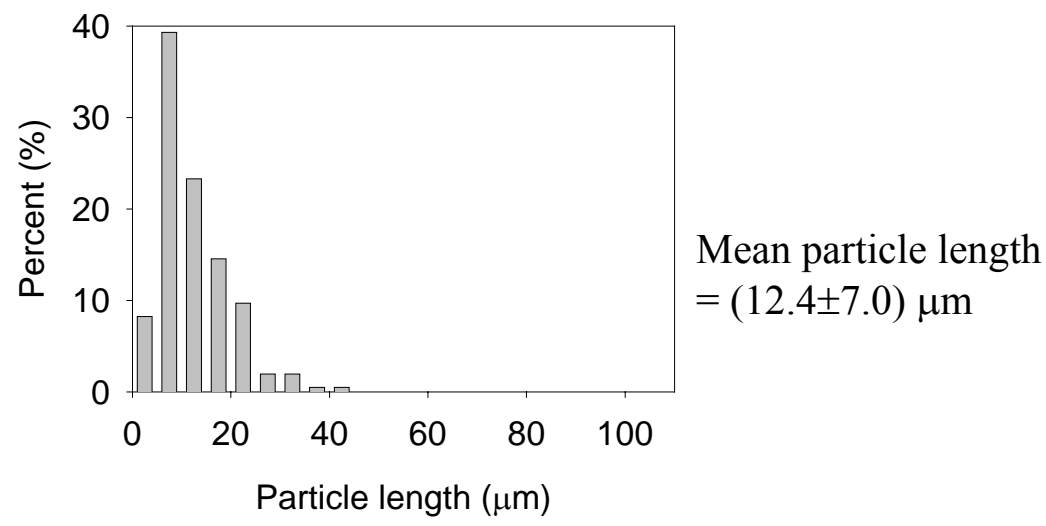

(c)

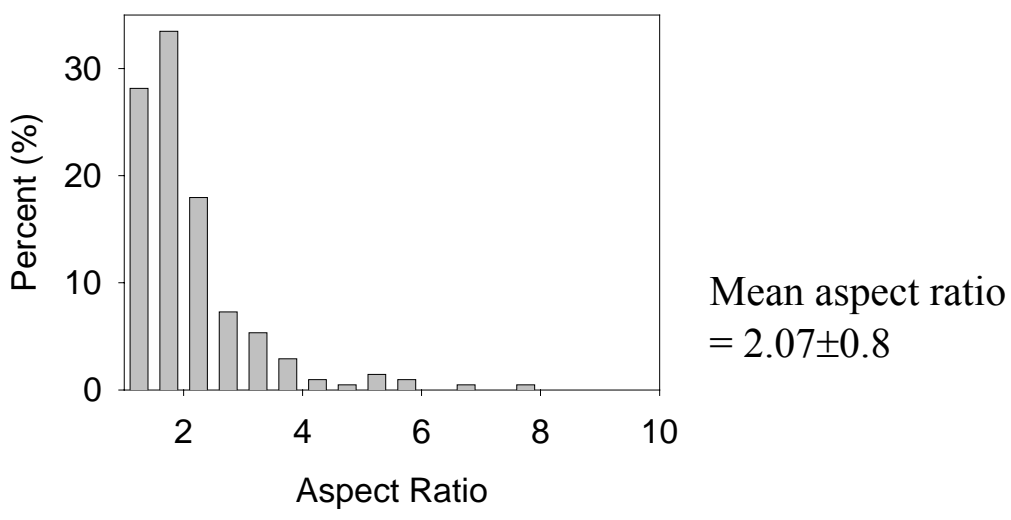

Figure 5.6 Particles generated with 320 grit abrasive paper made crosshatched stainless steel surface. (a) SEM micrograph, (b) Distribution of particle length, (c) Distribution of particle aspect ratio. 


\subsection{Effect of surface-texture pattern on particle shape}

Particles generated from the unidirectional steel surfaces have large aspect ratios compared to the particles generated from the crosshatched surface. Unidirectional surface textures were found to generate more elongated particles (Figures 5.3-5.4) and crosshatched surface textures generated round-like particles (Figures 5.5-5.6). In Figure 5.7, we further compare the aspect-ratio distribution for the particles generated from unidirectional and crosshatched surfaces. Over $85 \%$ of the particles in the group with elongated shapes are the ones with aspect ratio larger than 2. On the other hand, a portion of more than $75 \%$ of the group with round-like shapes has an aspect ratio less than 2. Evidently, the different types of surface textures produce wear particles with two distinct aspect ratios. This result demonstrates the feasibility of generating UHMWPE wear particles with different shapes through surface texturing.

The basic underlying concept is that the size and shape of the wear particles can be controlled by the cutting edges and by the geometric constraints superimposed on the volume defined by the groove edges. The unidirectional texture presents an array of parallel cutting edges on the stainless steel surface. During sliding, these edges cut into the UHMWPE and produce elongated particles. The crosshatched texture, on the other hand, has diamond-shaped edges with an internal volume. While the edges cut into the soft elastic UHMWPE material, the diamond-shaped volume of the intersection of the grooves controls the round particle size. 

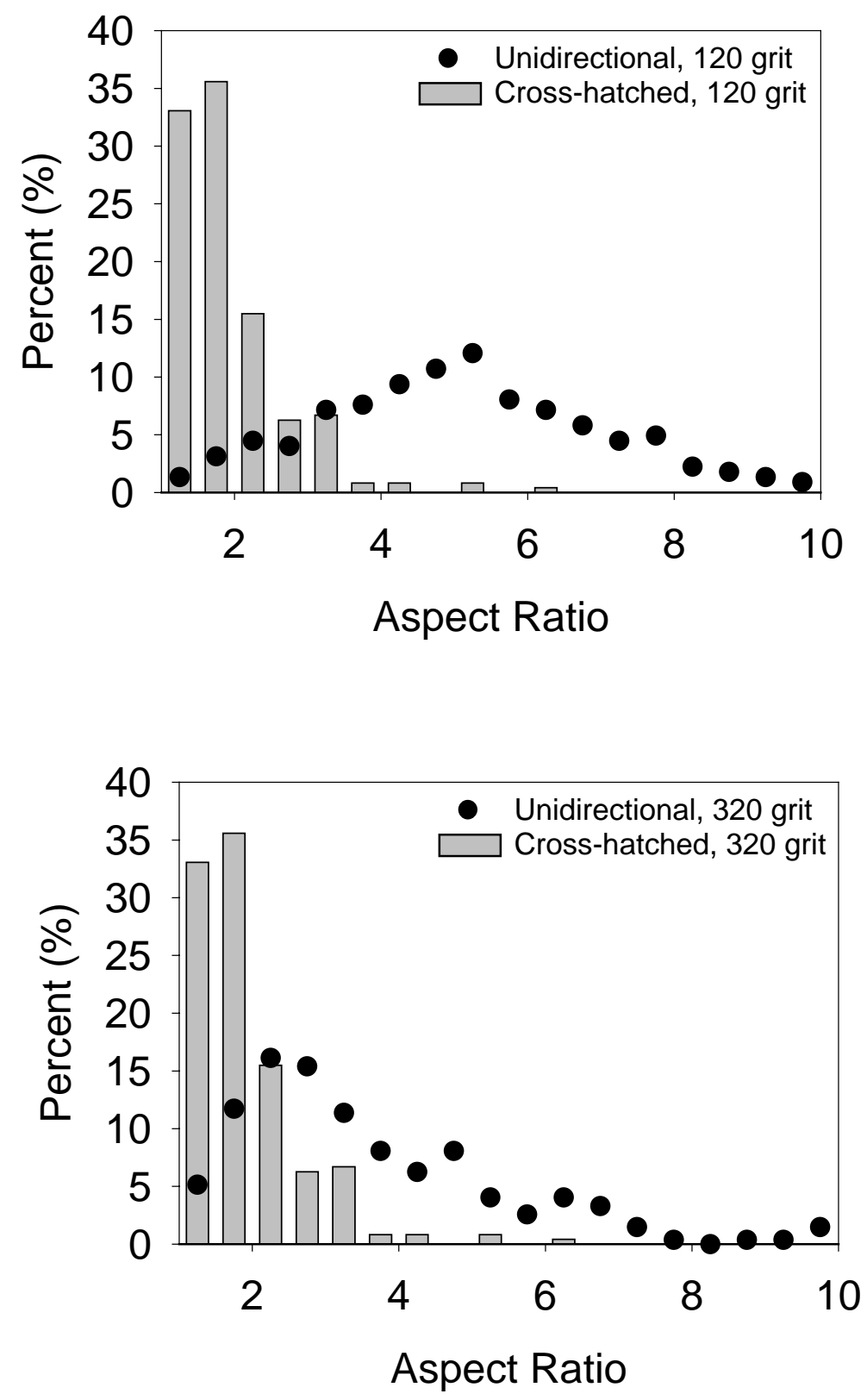

(Uncertainty of the measurement comes from the image measurement of particles which is estimated to be less than $5 \%$ of the mean value)

Figure 5.7 Comparison of particle aspect-ratio distribution from abrasive paper made stainless steel surfaces with different surface-texture styles. 


\subsection{Effect of surface-texture roughness on particle size}

The rougher surfaces were prepared by the abrasive papers that are bonded with larger $\mathrm{SiC}$ powders. The roughness ( $\mathrm{Ra}$ : mean of the deviation of height to the average height of the surface profile) was measured perpendicular to the groove direction for the unidirectional steel surfaces. From Table 5.2, it is seen that for the unidirectional surface textures, when the surface roughness was increased from 0.223 $\mu \mathrm{m}$ to $0.524 \mu \mathrm{m}$, the mean particle length increased from $31.6 \mu \mathrm{m}$ to $47.0 \mu \mathrm{m}$. For the crosshatched surface textures, the roughness was measured in the diagonal direction to the grooves. When the surface roughness was increased from $0.175 \mu \mathrm{m}$ to $0.413 \mu \mathrm{m}$, the mean particle length increased from $12.4 \mu \mathrm{m}$ to $22.7 \mu \mathrm{m}$. However, the mean aspect ratios of the particles are similar for each kind of surface texture. Figure 5.8 further compares the distribution of the length of the particles generated from the surface textures with different roughness. The surface profile is magnified while a rougher $\mathrm{SiC}$ abrasive paper is used to prepare the surface texture. The larger particles are generated because larger cutting features are applied in the cutting process of particle generation.

Table 5.2 Effect of roughness of stainless steel surfaces on particle dimensions.

\begin{tabular}{|l|l|l|l|l|}
\hline & 120 grit, // & $\mathbf{1 2 0}$ grit, \# & $\mathbf{3 2 0}$ grit, // & 320 grit, \# \\
\hline Surface roughness Ra $(\mu \mathrm{m})$ & $0.524 \pm 0.21$ & $0.413 \pm 0.18$ & $0.223 \pm 0.12$ & $0.175 \pm 0.10$ \\
\hline Mean particle length $(\mu \mathrm{m})$ & $47.0 \pm 19.0$ & $22.7 \pm 10.6$ & $31.6 \pm 16.7$ & $12.4 \pm 7.0$ \\
\hline Mean particle width $(\mu \mathrm{m})$ & $14.8 \pm 3.2$ & $12.7 \pm 3.0$ & $10.1 \pm 2.8$ & $6.4 \pm 2.4$ \\
\hline Mean particle aspect ratio & $3.44 \pm 1.7$ & $1.89 \pm 0.7$ & $3.60 \pm 1.9$ & $2.07 \pm 0.8$ \\
\hline
\end{tabular}

Note. //: unidirectional texture; \#: cross-hatched texture. (mean \pm standard deviation) 

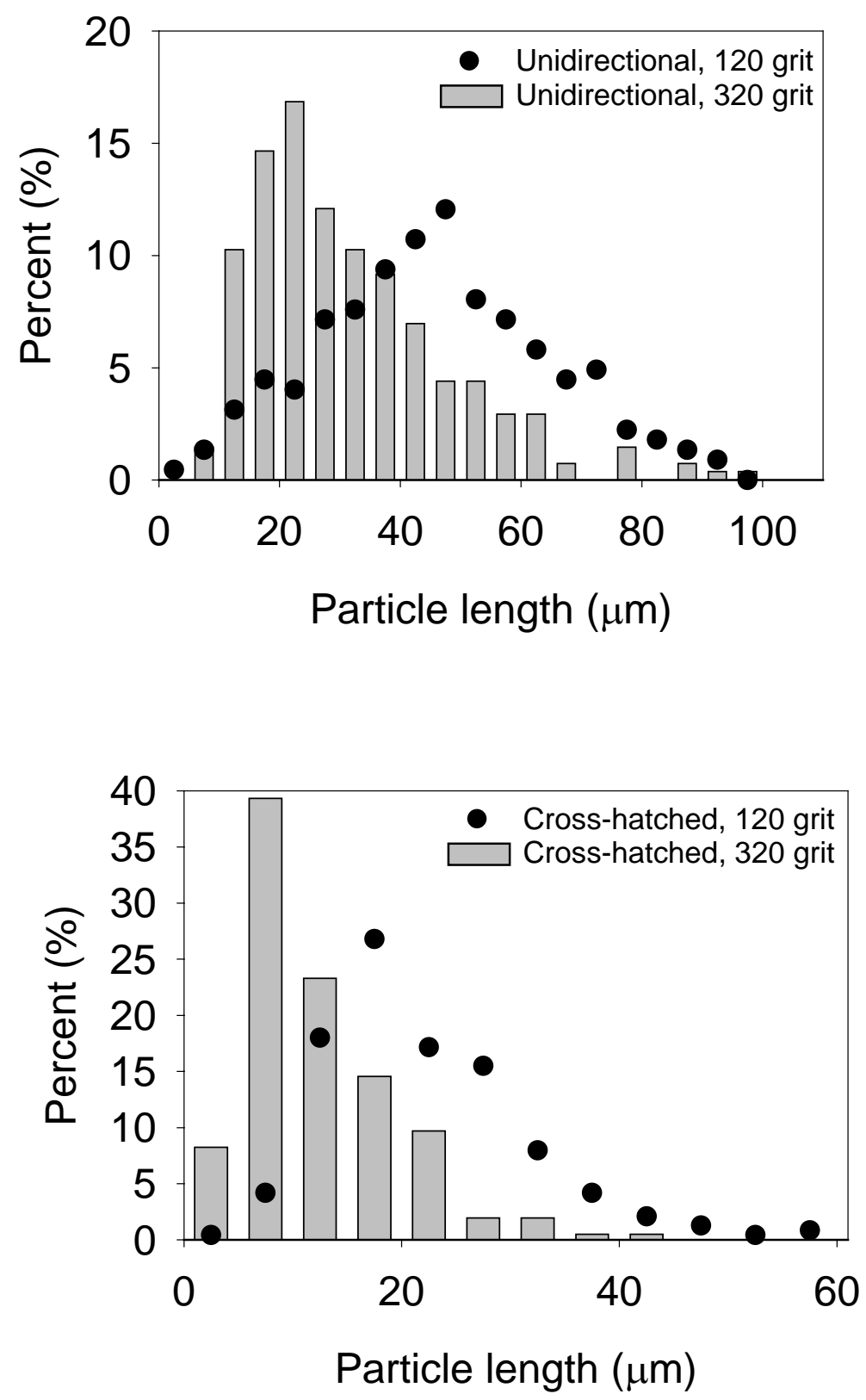

(Uncertainty of the measurement comes from the image measurement of particles which is estimated to be less than $5 \%$ of the mean value)

Figure 5.8 Comparison of particle-length distribution from stainless steel surfaces made by different grades of abrasive papers. 


\subsection{Particle generation with diamond-tip scratched surfaces}

\subsubsection{Preparation of diamond-tip scratched stainless steel surfaces}

Stainless steel 316 plates (38 $\mathrm{mm}$ in diameter and $6.35 \mathrm{~mm}$ in thickness) were polished to a surface roughness of less than $0.025 \mu \mathrm{m}$. A $90^{\circ}$ conical diamond tip was mounted on a scratching tester. The tester is capable of moving in the $\mathrm{X}, \mathrm{Y}$, and $\mathrm{Z}$ directions with displacement resolution of $(1 \pm 0.2) \mu \mathrm{m}$. The stainless-steel plate was fixed and leveled on the stage. The diamond tip was lowered in the $\mathrm{Z}$ direction to be in contact with the polished steel surface. The contact point was defined when the contact force was measured and monitored by a force-recording system. We further lowered the diamond tip to indent the steel surface with a $3 \mu \mathrm{m}$ interference depth. Then the diamond tip was moved a pre-defined distance by a computer in the Ydirection to scratch the steel surface with a sliding speed of $1 \mathrm{~mm} / \mathrm{s}$. Purified paraffin oil was used here as a lubricant. After the scratching, the diamond tip was lifted. Then the diamond tip moved in the $\mathrm{X}$ and the $\mathrm{Y}$ directions over specified distances and the scratching process was repeated. A schematic representation of the scratching process is shown in Figure 5.9. Numerous scratches were made on the stainless-steel surface with various scratching lengths and depths. The textured plate was then cleaned ultrasonically in solvents and detergents, followed by rinsing with de-ionized water and drying with nitrogen.

The diamond-tip scratched steel surfaces were then mounted on the linear reciprocating wear tester. The particle generation process proceeded and the particles were collected and analyzed. 


\section{Programmable sliding w/ controlled interference depth}

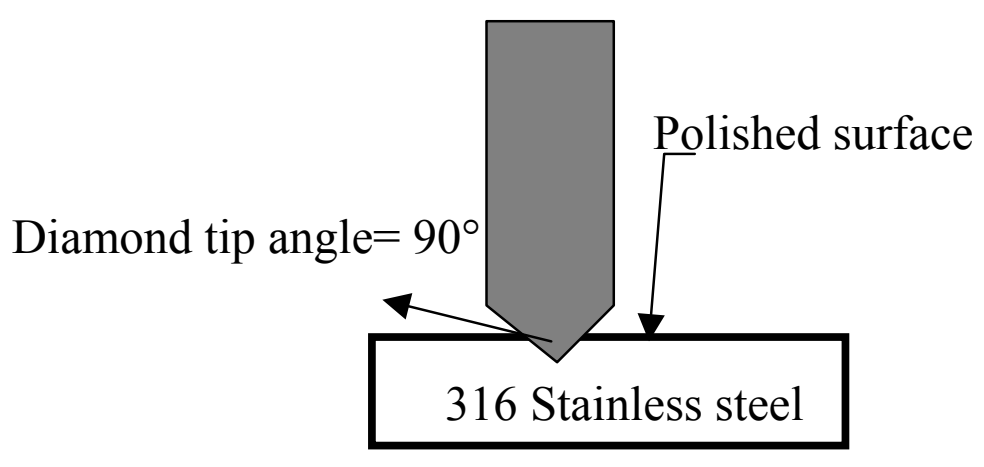

\section{Procedure:}

- $\quad$ Level the steel plate

- $\quad$ Detect the diamond tip-steel plate contact point by force sensor

- Program the actuator with interference depth, travel distance and speed

- $\quad$ Use pure paraffinic oil as lubricant

- $\quad$ Start scratching the surface

- $\quad$ Clean the surface by detergent with ultrasonic bath

Figure 5.9 Procedure of surface texturing by diamond scratching. 


\subsubsection{Results}

Two diamond-tip scratched steel surfaces were prepared with an interference depth of $3 \mu \mathrm{m}$. To discuss the effect of scratched dimensions on particle dimensions, two scratched lengths were chosen for comparison. One had linear scratches over the whole steel surface. The other one had a $60 \mu \mathrm{m}$ scratching distance with a distance of $60 \mu \mathrm{m}$ between two scratches along the scratching direction. The distance between adjacent scratches for both surfaces was $300 \mu \mathrm{m}$. Figure 5.10 shows the SEM micrographs of both diamond-tip scratched steel surfaces.

The wear experiments were conducted under the following conditions: normal load is $98 \mathrm{~N}$ (nominal contact pressure $\approx 3 \mathrm{MPa}$ ), stroke length is $19.05 \mathrm{~mm}$, frequency is $1.5 \mathrm{~Hz}$; average sliding velocity is $57.2 \mathrm{~mm} / \mathrm{s}$, duration is $2 \mathrm{~h}$. The UHMWPE pin was rubbed against the diamond-tip scratched surface in the direction perpendicular to the scratched grooves as shown in Figure 5.10. The SEM micrographs of the UHMWPE particles, and the distributions of particle length and particle aspect ratio for the two cases are shown in Figures 5.11 and 5.12. The mean particle lengths \pm standard deviations and aspect ratios \pm standard deviations for both cases are listed in Table 5.3.

Table 5.3 Results of particle generation with diamond-tip scratched steel surfaces.

\begin{tabular}{|l|c|c|}
\hline & Linear scratched surface & Scratched distance $=60 \mu \mathrm{m}$ \\
\hline Mean particle-length $(\mu \mathrm{m})$ & $41.7 \pm 10.2$ & $24.8 \pm 4.8$ \\
\hline Mean particle-width $(\mu \mathrm{m})$ & $14.6 \pm 3.9$ & $13.7 \pm 3.1$ \\
\hline Mean particle aspect-ratio & $2.85 \pm 0.62$ & $1.81 \pm 0.32$ \\
\hline
\end{tabular}




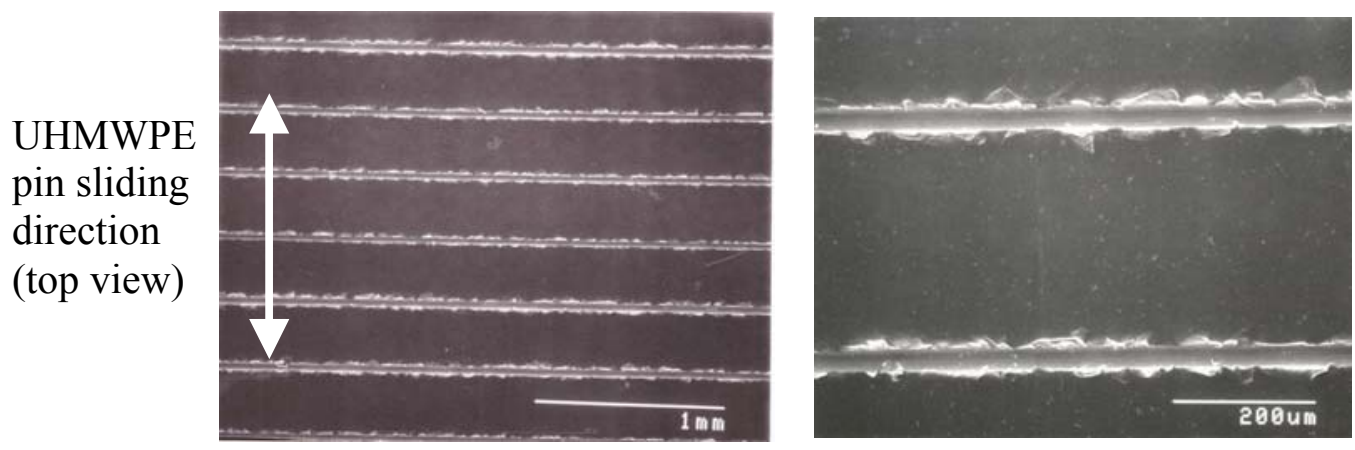

(a)

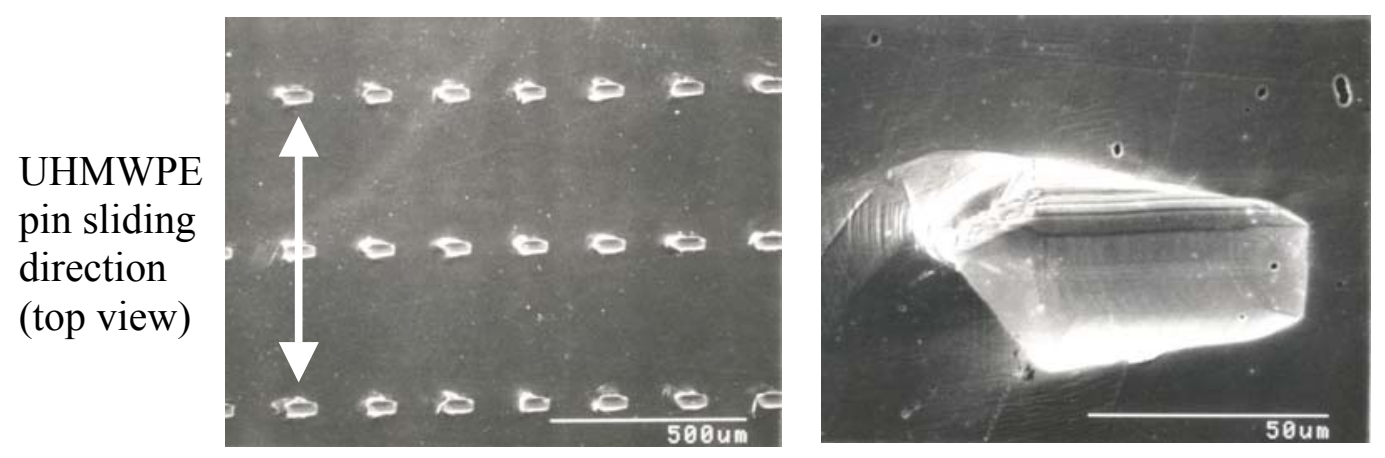

(b)

Figure 5.10 SEM micrographs diamond-tip scratched surfaces: (a) with linear scratches, (b) with a $60 \mu \mathrm{m}$ scratched length. 
(a)

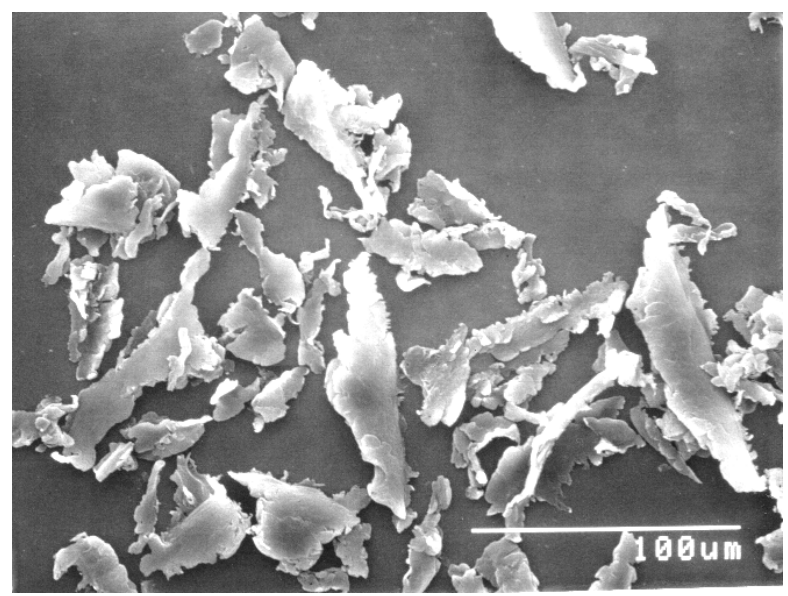

(b)

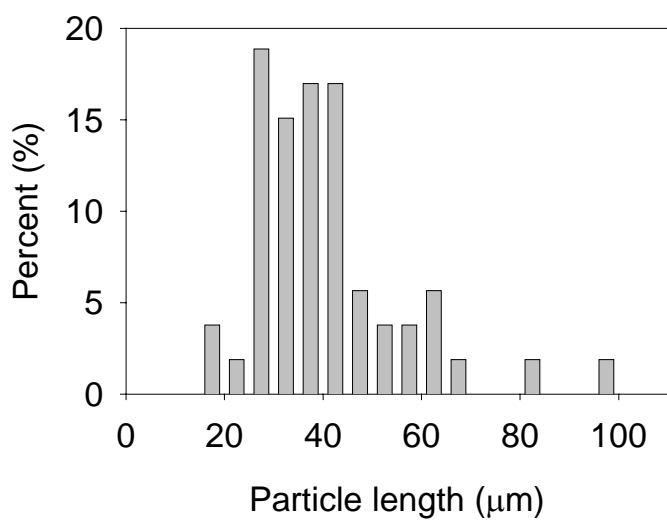

Mean particle length

$=(41.7 \pm 10.2) \mu \mathrm{m}$

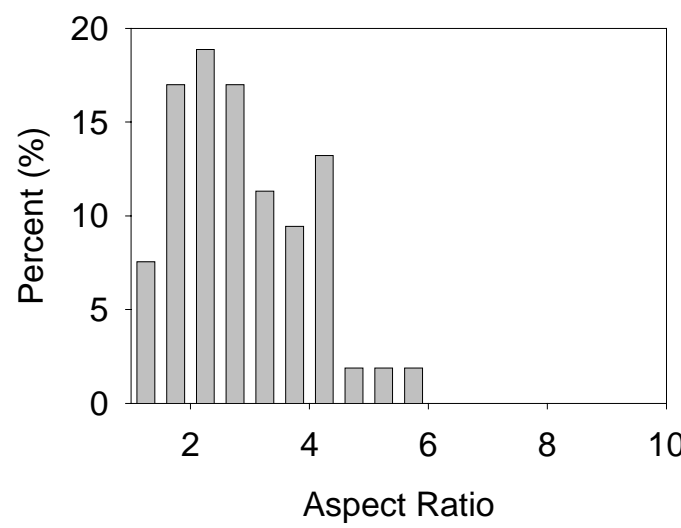

Mean aspect ratio

$=2.85 \pm 0.62$

Figure 5.11 Particles generated with the diamond-tip scratched stainless steel surface (linear type) (a) SEM micrograph, (b) Distribution of particle length, (c) Distribution of particle aspect ratio. 
(a)

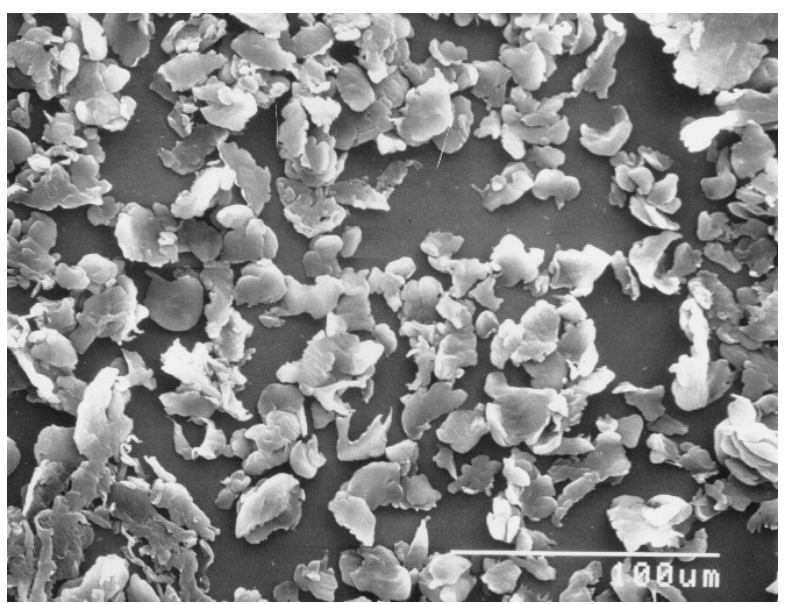

(b)

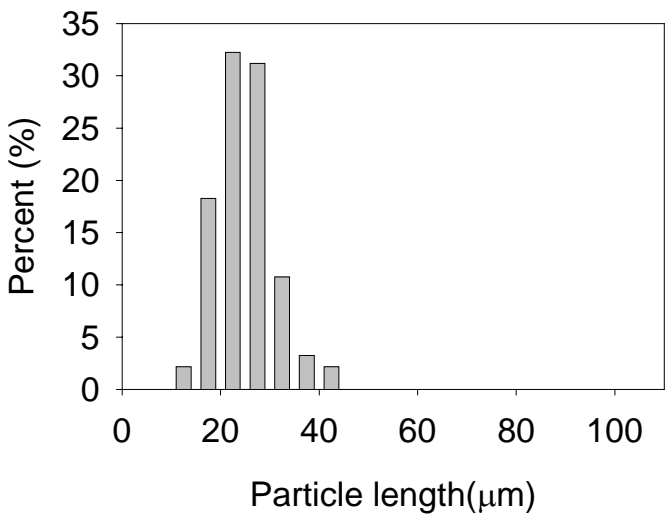

Mean particle length $=(24.8 \pm 4.8) \mu \mathrm{m}$

(c)

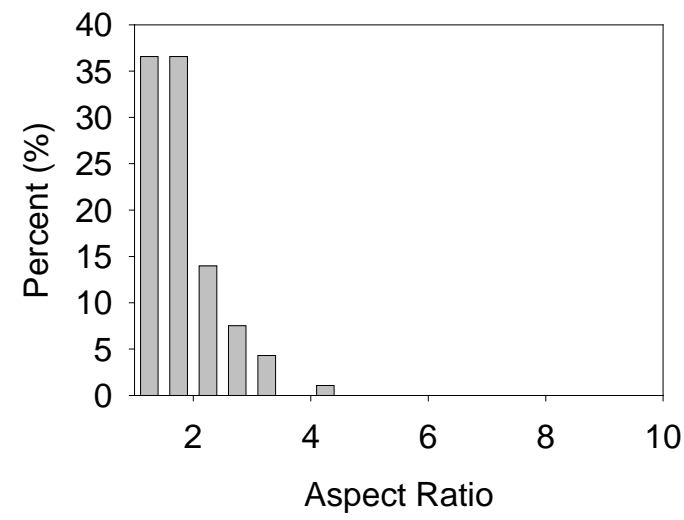

Mean aspect ratio

$=1.81 \pm 0.32$

Figure 5.12 Particles generation with the diamond-tip scratched stainless steel surface (scratched distance $=60 \mu \mathrm{m}$ ) (a) SEM micrograph, (b) Distribution of particle length, (c) Distribution of particle aspect ratio. 


\subsection{Effect of surface uniformity on particle distribution}

When the surface textures are prepared by $\mathrm{SiC}$ abrasive papers, only the surface roughness, and surface-texture patterns can be changed by using different grades of abrasives and different abrading directions. This process produces a wide distribution of cutting edges. By applying a diamond-tip scratching technique with displacement control, the uniformity of the surface features is significantly improved. Figure 5.13 compares the surface profiles (meassured by a surface profilometer) of the linear-type surface features made with $\mathrm{SiC}$ abrasive appers and with the diamond-tip scratching technque, respectively. The tracing direction is perpendicular to the groove direction. The standard deviations of particle length generated are shown in Figure 5.14. Results include the particle populations from the surface textures made with $\mathrm{SiC}$ abrasive papers and from the surface textures made with the diamond-scratching process. With similar mean particle length, diamond-scratched steel surfaces do produce particles with narrower distribution. However, the improvement is not sufficient to generate particle populations with fully separate particle distributions.

One reason leading to the improvement is that the depth of the groove can be controlled by the penetration depth of diamond tip during the scratching process. However, positive material bumps along both sides of the grooves are produced by the material plastic deformation process. Thus there exists some difficulty to further precisely control these positive bumps which act as cutting edges. The results also show that when the scratched distance was reduced to $60 \mu \mathrm{m}$ from the linear scratches, the particle length decreased. Ideally, the particle length should be equal to the cutting- 
edge length defined by the scratched distance in this example. However, imperfection of the scratch-induced cutting edges results in smaller cutting-edge lengths in real situations. To further narrow the particle distribution, one needs to be able to fabricate cutting edges with precisely defined dimensions, especially when micrometer scaled features are needed.

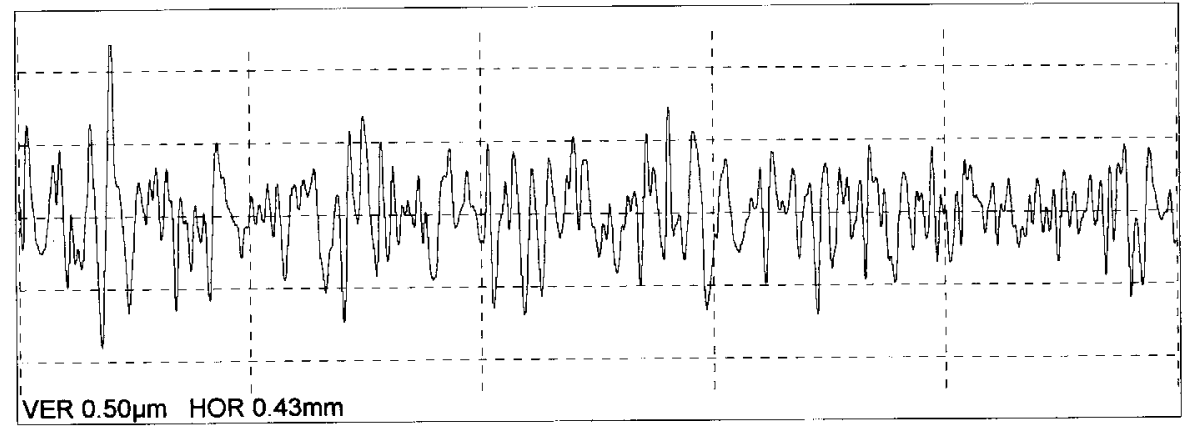

(a)

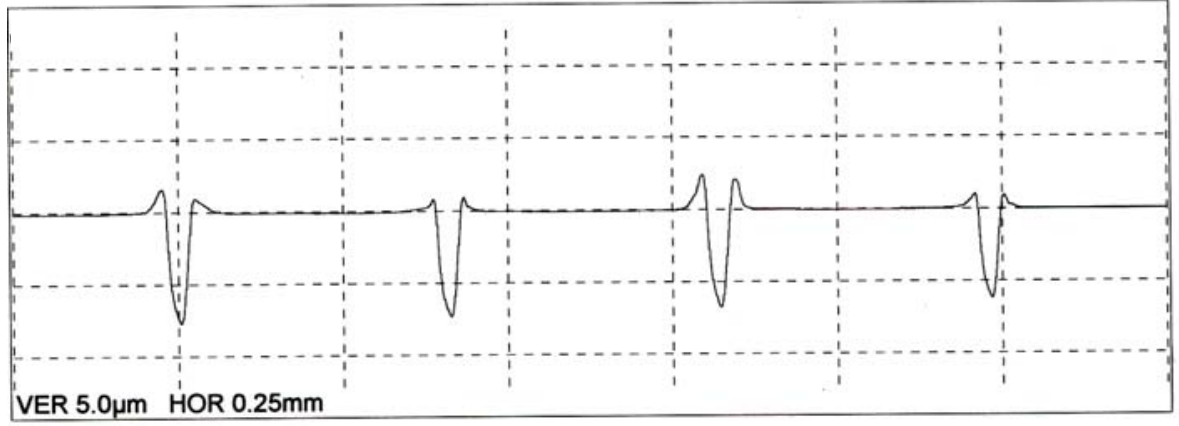

(b)

Figure 5.13 Examples of surface profiles for (a) $\mathrm{SiC}$ abrasive-paper made stainless steel surface, and (b) Diamond-tip scratched steel surface. 


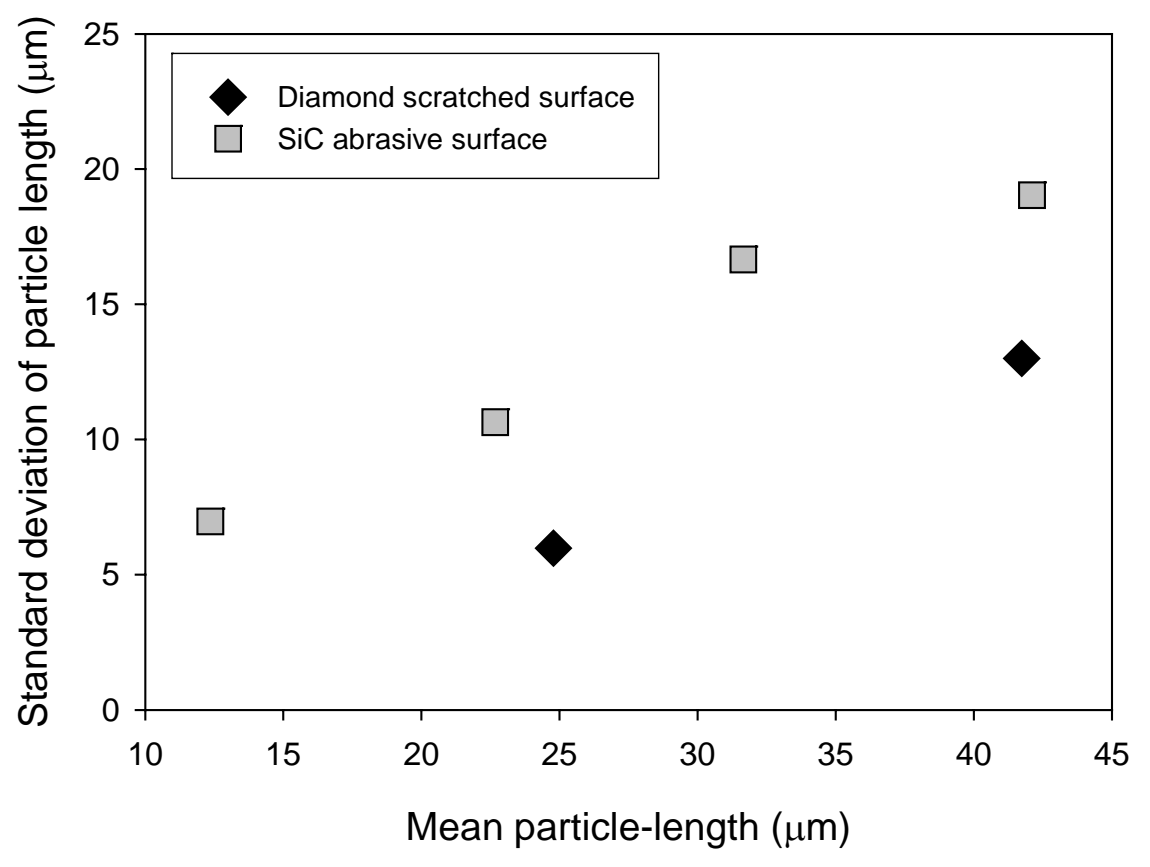

(Uncertainty of the particle-length measurement is denoted as standard deviation in the plot)

Figure 5.14 Comparison of standard deviation and mean particle length for different particle-size populations made with surfaces made with $\mathrm{SiC}$ abrasive papers and with diamond-tip scratching process. 


\section{CHAPTER 6}

\section{SURFACE TEXTURING BY MICROFABRICATION}

\subsection{Introduction}

The particle distribution appears to be strongly related to the uniformity of the textured surfaces. The uniformity of the textured surfaces needed to be further improved. Microfabrication techniques for semiconductor and micro-electromechanical devices (MEMs) manufacturing have been developed to fabricate features with a sub-micrometer resolution [96]. By preparing surface textures with microfabrication techniques, the dimensions of each cutting feature on the surface can be precisely controlled. In this chapter, we describe the procedures to texture surfaces by lithography and chemical etching processes. The dimensions of the surface features are measured by scanning electron microscopy (SEM), atomic force microscopy (AFM) and profilometer techniques.

\subsection{Surface texture with wedge features}

We proposed to fabricate wedge-shaped features on the surface for the microcutting process. With the controlled length and sharpness of the edge, the normal load during the particle-generation process can be adjusted to achieve the desired deformation of UHMWPE by penetrating the wedge edge into the material. By 
adjusting the height of the wedge feature and the distance between adjacent features, the confined volume can be determined. In the remaining sections of this chapter, the detailed microfabrication processes are described for fabricating a wedge-type surface texture.

\subsection{Microfabrication process}

Based on the principles of surface-texture design, microfabrication is applied to make effective surface-texture features for particle generation. The procedures include the design of surface-texture patterns on a two-dimensional photomask, transfer of patterns to the material surface by lithography, etching of materials to generate three-dimensional structures, and the surface treatments to enhance wear resistance. Figure 6.1 shows schematically the microfabrication process to produce the wedge features on the silicon material. The technical details are discussed in the sections below.

\subsubsection{Substrate material}

Single crystal silicon was chosen as the substrate material. Polished silicon wafers with type $\mathrm{P}$ and (100) orientation were prepared as the base material for the texturing process. The dimensions of a silicon wafer are $50.8 \mathrm{~mm}$ diameter with 0.3

mm thickness. An additional process for microfabrication of features on stainless steel is described in Appendix B. 


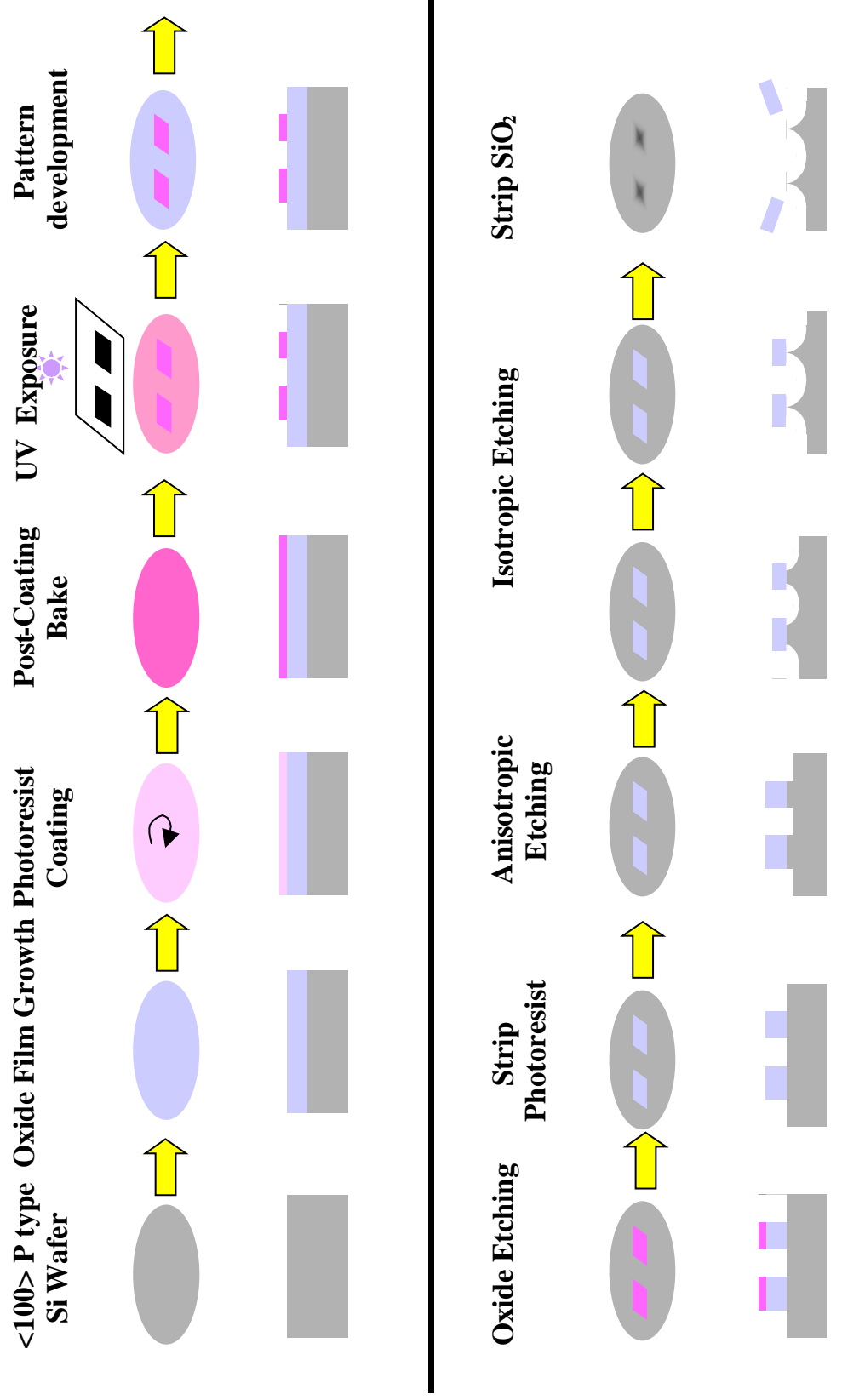

Figure 6.1 Schematic of microfabrication process to generate wedge features on silicon. 


\subsubsection{Masking material}

In order to fabricate wedge features on the silicon substrate by a chemical process, we need to put a layer of masking material on the silicon surface. In our application, silicon dioxide is chosen because of its lower etching rate by the chemical etchant that is applied to etch silicon at the final stage. Thermal oxidation of silicon is easily achieved by heating the substrate to the range of $900{ }^{\circ} \mathrm{C}$ to $1200{ }^{\circ} \mathrm{C}$. The atmosphere in the furnace where oxidation takes place can either contain pure oxygen or water vapor. Both of these molecules diffuse easily through the growing silicon dioxide layer at these high temperatures. Oxygen arriving at the silicon surface can then combine with silicon to form silicon dioxide. A wet oxidation process is applied in this study. Hydrogen and excess oxygen gases flow into the furnace and form the water vapor. The high-temperature water vapor then reacts with silicon to form silicon dioxide

The relationship between the silicon dioxide thickness and the temperature, the time required for the process can be found in the literature [96]. A layer of silicon dioxide with $1 \mu \mathrm{m}$ thickness was grown in our application under $1100{ }^{\circ} \mathrm{C}$ for $135 \mathrm{~min}$. The thickness of the silicon dioxide film was measured by Nanospec/AFT (Nanometrics). The instrument uses a scanned wavelength illumination source to determine the thickness and refractive index of thin films. The spectrophotometerhead scans generate a corrected spectrum by computing a ratio to a bare silicon reference previously stored. The resulting spectral data are used to determine the exact film thickness corresponding to the interference pattern. 


\subsubsection{Pattern design and transfer}

Two-dimensional patterns were designed. The dark features on the pattern (Figure 6.1, under UV exposure step) represent the protected area from the etching process. The patterns were then transferred to a chromium coated quartz photomask by the direct-writing process at the photomask manufacturer. The critical tolerance resolution of the photomask used is $1 \mu \mathrm{m}$.

The silicon wafer with silicon dioxide superlayer was heated on a hot plate at $205^{\circ} \mathrm{C}$ for one min. This is the dehydration process to remove the water remaining on the material surface. The wafer was then spin-coated with 1,1,1,3,3Hexamethyldislazane (HMDS) at $5000 \mathrm{rpm}$ for $30 \mathrm{~s}$. The purpose of this coating is to enhance the adhesion between the wafer surface and the later coated photoresist. Shipley 1813 photoresist was spin-coated on the silicon dioxide surface at $5000 \mathrm{rpm}$ for $30 \mathrm{~s}$. The silicon wafer was then baked on a hot plate at $100{ }^{\circ} \mathrm{C}$ for $2 \mathrm{~min}$ to enhance the stability of the coated photoresist. The average thickness of the photoresist layer is $(1.3 \pm 0.2) \mu \mathrm{m}$.

The dark-featured photomask was then placed in contact on the photoresist surface at a mask aligner for the following photolithography process. It was exposed to a UV source to decompose the surrounding polymer surface leaving the positive rectangular patterns that were covered by the dark features on the photomask. A postbake process was carried out at $100{ }^{\circ} \mathrm{C}$ for $90 \mathrm{~s}$ to accelerate and accomplish the decomposition of photoresist. In order to fully remove the decomposed photoresist, the wafer surface was rinsed by a Shipley photoresist-developer for $30 \mathrm{~s}$ at room 
temperature followed by water rinse and blow dry processes. Up to this stage, the patterns were transferred on the photoresist layer on the silicon dioxide film.

The resulting positive photoresist patterns were now acting as the protecting layer for the following silicon-dioxide etching process. The etchant used to etch the silicon dioxide contains hydrofluoric acid $(\mathrm{HF})$ and nitric acid $\left(\mathrm{HNO}_{3}\right)$ with a volume ration of 6:1. The wafer was then carefully placed on a bath of the etchant at room temperature for $5 \mathrm{~min}$ to remove the silicon dioxide areas that are not protected by the photoresist. At this stage, the patterns have been transferred to the masking layer of silicon dioxide for the following silicon etching process.

\subsubsection{Fabrication of surface features}

Up to this step, the two-dimensional patterns were made on the masking material (silicon dioxide). The next step was to convert the two-dimensional patterns into three-dimensional structures on the silicon substrate. To construct the wedge geometry of the surface feature, etching of the silicon in the vertical and horizontal directions is necessary. Etching of the silicon in the vertical direction controls the height of the wedge feature. In order to form the sharp linear edge of the wedge, the silicon material under the masking silicon dioxide should be etched horizontally from outside towards to the center.

To achieve control of the edge angle, a combination of isotropic chemical etching and anisotropic etching in the vertical direction were used. Actually, an anisotropic etching process has a high ratio of the etching rate in the vertical direction 
to the etching rate in the horizontal direction. Anisotropic etching should be first carried out to create the height of the feature with limited etching in the horizontal direction. Then the isotropic etching process etches the silicon substrate with the same etching rate in any direction. We stopped the etching process when the silicon substrate under the masking silicon dioxide was etched away. This technique has been used by others to fabricate knife features for applications in telecommunication [97].

The final height and edge angle of the surface texture are dependent on the etching period of anisotropic and isotropic etching process. Figure 6.2 shows this process schematically.

With a known etching rate of the isotropic etching process $R_{\mathrm{i}}$, vertical anisotropic etching rate $R_{\mathrm{a}, \mathrm{z}}$, and the ratio of the vertical etching rate to the horizontal etching rate of the anisotropic etching process $C_{\mathrm{a}}$, the etched distances in the vertical and horizontal directions for both etching processes can be calculated. The final height of the surface features and the edge angles can be determined by the durations $t_{\mathrm{a}}$ and $t_{\mathrm{i}}$ for the anisotropic and isotropic etching processes:

$$
\begin{aligned}
& h_{\mathrm{c}}=R_{\mathrm{a}, \mathrm{z}} \cdot t_{\mathrm{a}}+R_{\mathrm{i}} \cdot t_{\mathrm{i}} \\
& w_{\mathrm{c}}=\left(c_{\mathrm{a}} R_{\mathrm{a}, \mathrm{z}}\right) \cdot t_{\mathrm{a}}+R_{\mathrm{i}} \cdot t_{\mathrm{i}} \\
& \theta=\tan ^{-1} \frac{w_{\mathrm{c}}}{h_{\mathrm{c}}}
\end{aligned}
$$

where $h_{\mathrm{c}}$ is the final height of the feature, $w_{\mathrm{c}}$ is the width of the feature and $\theta$ is the half angle of the edge. 

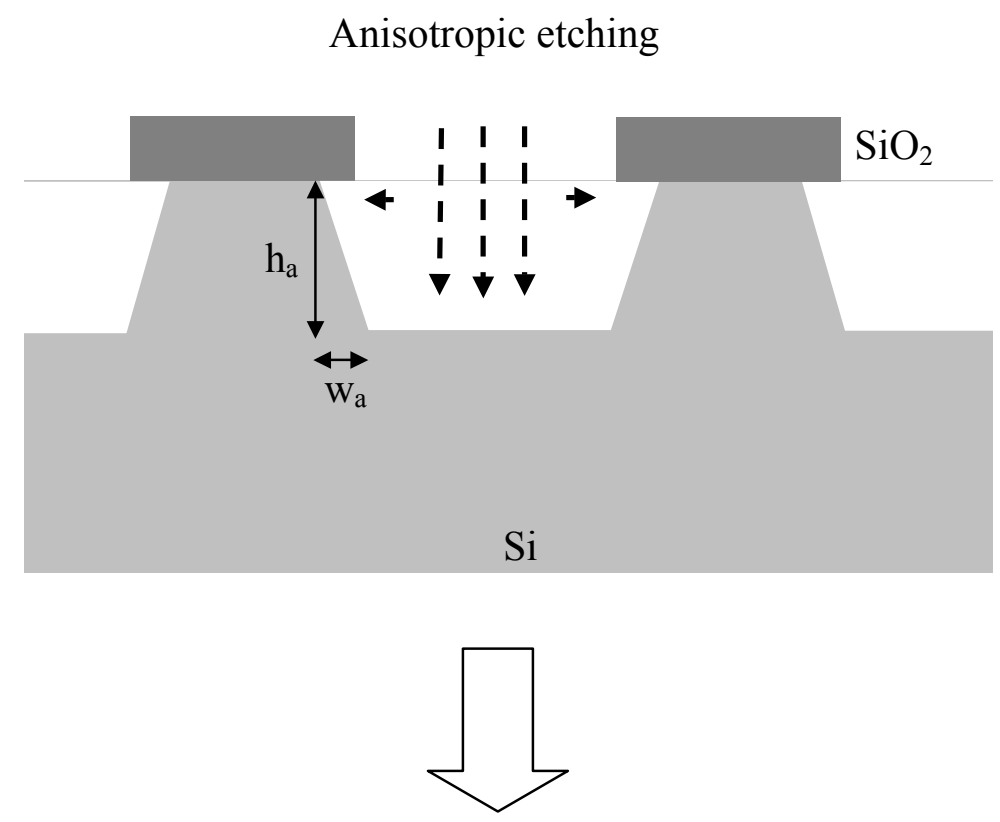

Isotropic etching

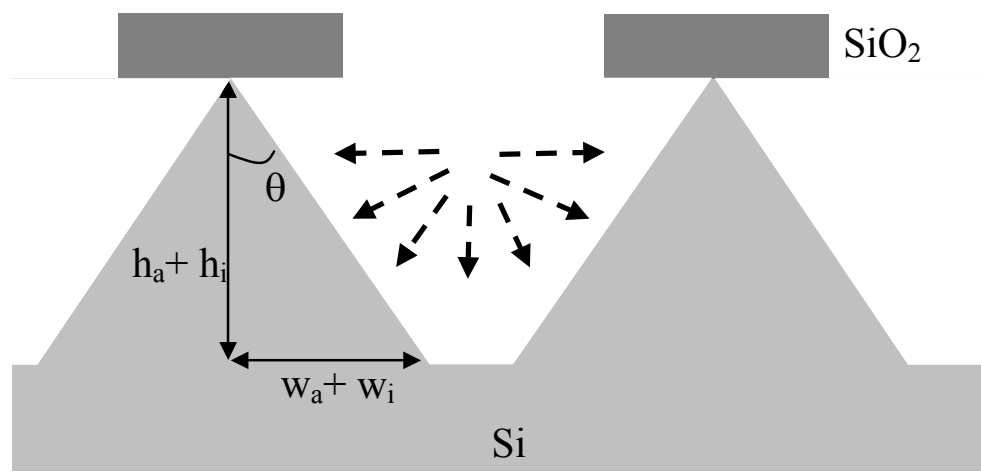

Figure 6.2 Schematic representation of the wedge geometry controlled by anisotropic and isotropic etching processes. 
The anisotropic etching process was carried out with a reactive ion etcher (RIE) made by Trion Corporation. $\mathrm{SF}_{6}$ gas was used to etch the silicon material. The process was operated under a gas pressure of $40 \mathrm{~Pa}$ with 100 Watt power. The etching rate in the vertical direction is $1 \mu \mathrm{m} / \mathrm{min}$, and the ratio of the vertical etching rate to the horizontal etching rate of the anisotropic etching process $c_{\mathrm{a}}$ was about 0.1 . Then the isotropic etching process was performed by immersing the wafer into a mixture of strong acid solutions containing hydrofluoric acid, nitric acid, and acetic acid (a HNA etchant; volume ratio $\left.\mathrm{HF}: \mathrm{HNO}_{3}: \mathrm{CH}_{3} \mathrm{COOH}=8: 75: 17\right)$ at room temperature. The etching rate of silicon by HNA etchant was $0.7 \mu \mathrm{m} / \mathrm{min}$.

\subsubsection{Wear-resistant coating}

The surface texture plays the role of the cutting device for the particle generation process. To reduce failure and wear of the surface texture, a thin film of chromium was coated onto the surface of the surface features.

The wafer was mounted in a vacuum metal evaporator. The vapor pressure in the chamber was less than $2.67 \times 10^{-3} \mathrm{~Pa}$. The applied heating voltage and current was adjusted to reach the evaporation rate of $0.5 \mathrm{~nm} / \mathrm{s}$. A $(5 \pm 0.5) \mathrm{nm}$ layer of chromium was deposited on the surface of the features. 


\subsection{Measurement of the surface texture}

Scanning electron microscopy (SEM) was applied to determine the morphology of the wedge geometry. Figure 6.3 provides an example of the SEM observation of the features. The length and the width of the rectangle features can be measured from the SEM micrographs. To measure the height and the angle of the wedge features, an atomic force microscope was used. The three-dimensional geometry and the dimensions of a wedge feature can be measured as shown in Figure 6.4.
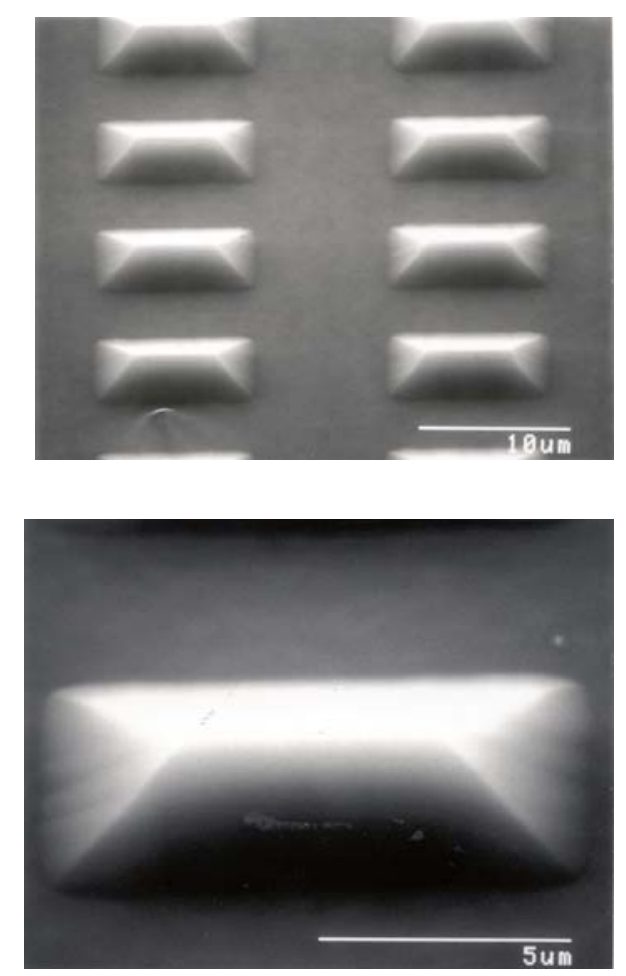

Figure 6.3 SEM micrographs of the wedge surface-features made by the microfabrication process. 


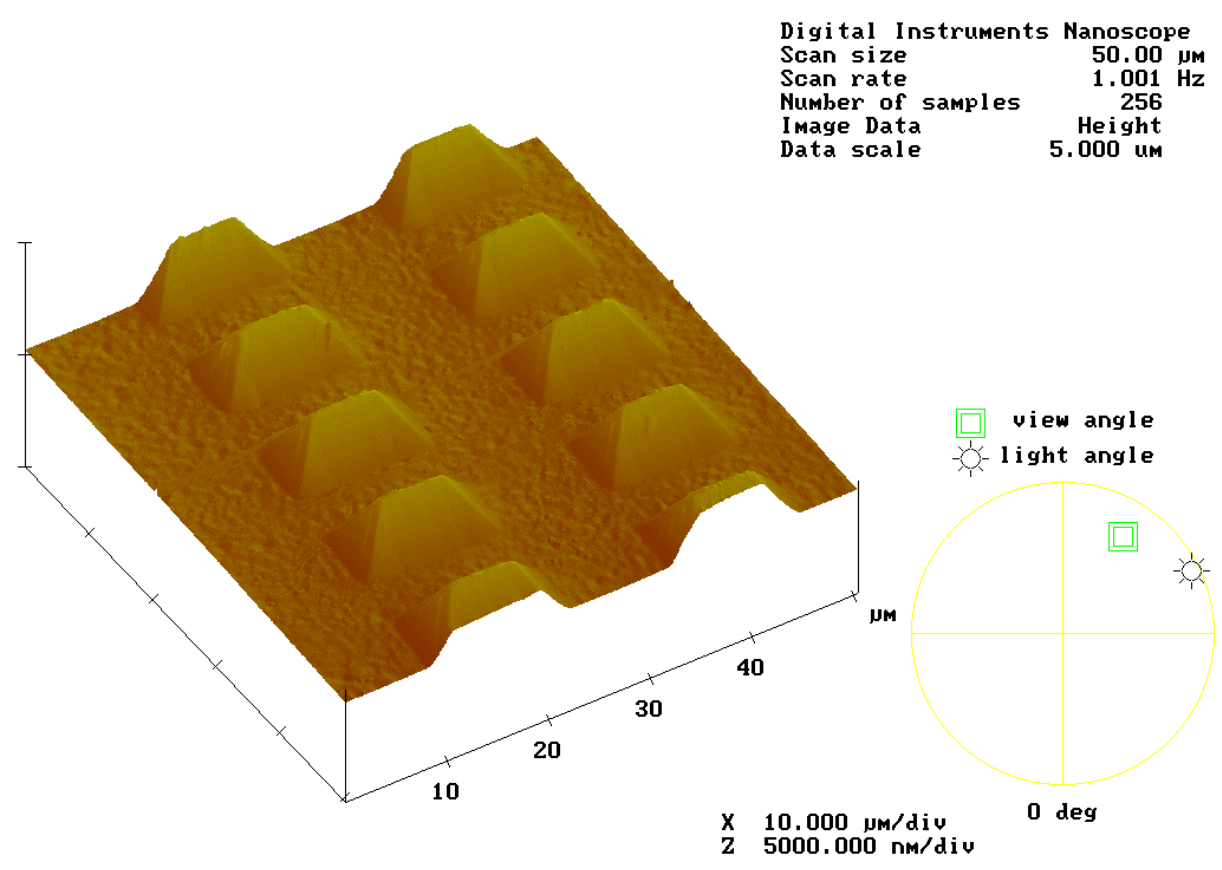

$5 \times 10 d$

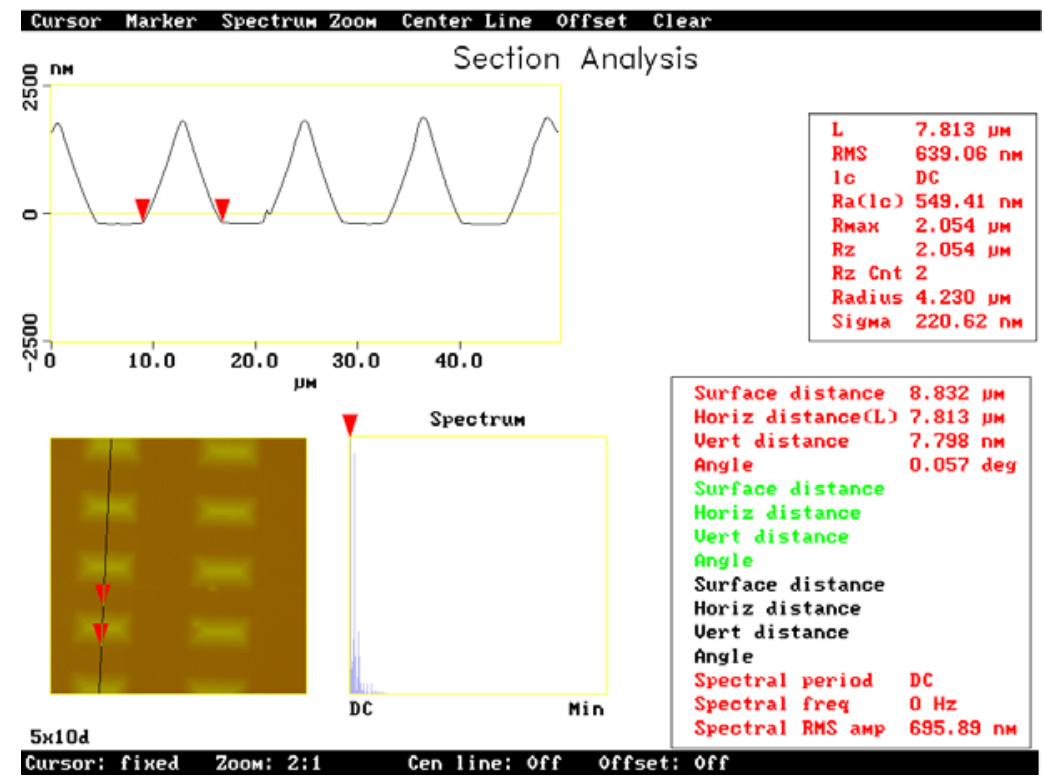

Figure 6.4 Measurement of the three-dimensional geometry of the wedge surface textures by atomic force microscopy (AFM). 


\section{CHAPTER 7}

\section{SURFACE-TEXTURE DESIGN AND GENERATION OF NARROWLY DiSTRIBUTED PARTICLES}

\subsection{Introduction}

In Chapter 6, the use of microfabrication techniques to produce precisely controlled surface-feature dimensions is discussed. Now it is feasible to study the relationship between surface-texture patterns (and their dimensions) and particle size and shape. This chapter describes the various designs and the resultant particle sizes and shapes produced.

\subsection{Surface-texture designs}

To design various surface textural patterns to generate different size and shape of particles, one has to develop some hypothesis of how they are related. It is assumed here that the critical parameters of the surface texture are the feature size and shape and the distances between the features that constitutes the overall pattern.

Figure 7.1 is a schematic of the surface texture used in this study. We can classify the parameters of the surface textures into two groups: (i) Dimensions of cutting edges: cutting-edge length $\left(L_{\mathrm{c}}\right)$, cutting-edge height $\left(H_{\mathrm{c}}\right)$, cutting edge width $\left(W_{c}\right)$ and half angle of the cutting-edge $(\theta)$; (ii) Arrangement of the cutting edges: pitch of the surface texture in the sliding distance $\left(D_{\mathrm{s}}\right)$, distance between adjacent 
cutting edges $\left(D_{\mathrm{i}}\right)$, distribution density (number of feature per unit area) of the cutting edges on the surface, and patterns of cutting-edge distributions.

Top view of the surface texture
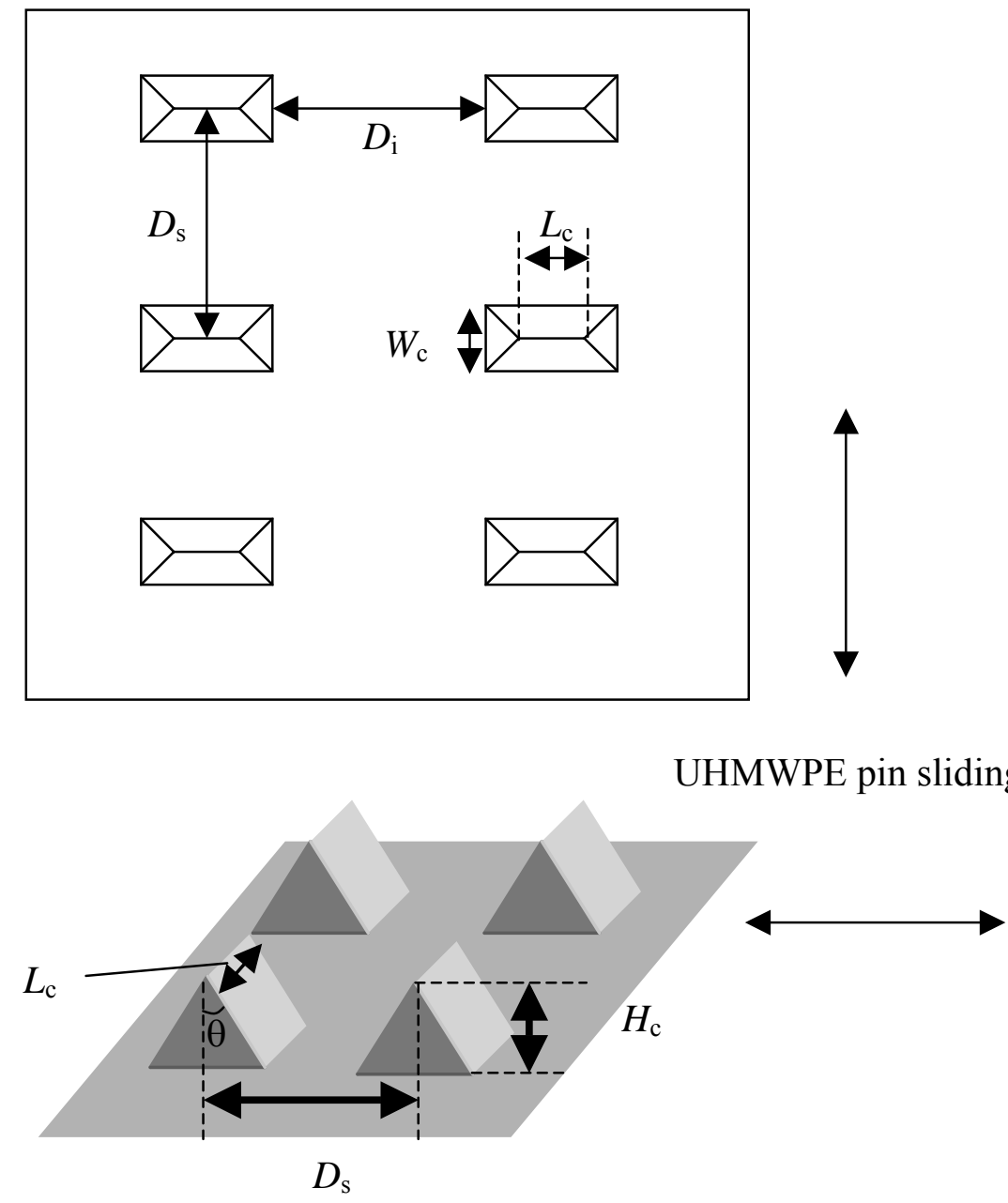

$L_{c}$ : cutting-edge length

$H_{\mathrm{c}}$ : cutting-edge height

$W_{\mathrm{c}}$ : cutting-edge width

$D_{\mathrm{s}}$ : pitch in the sliding direction

$D_{\mathrm{i}}$ : distance between adjacent features

Figure 7.1 Schematic of the surface texture with wedge features. 


\subsection{Surface textures fabricated}

Two types of surface textures were designed and microfabricated. Type A texture contains the wedge-shaped cutting edges lining up in a rectangular array with specified pitch $\left(D_{\mathrm{s}}\right)$ and distance between adjacent features $\left(D_{\mathrm{i}}\right)$ as shown in Figure 7.2. Type B texture has an offset arrangement of the features $\left(D_{\mathrm{s}} / 2\right)$ as shown in Figure 7.2. The distance between adjacent features was designed to be equal to the basal length of the cutting-edge.

The surface pattern parameters such as $D_{\mathrm{s}}$ and $D_{\mathrm{i}}$ were controlled by the photomask used in the microfabrication process. The density of the cutting edges on the surface (number of feature per unit area) was also calculated. These dimensions are listed in Figure 7.2 as well. Length and height of the cutting edges were measured after the microfabrication of the surface texture. Various cutting-edge lengths were designed to generate particles of different lengths. Different cutting-edge heights were included in an attempt to change the aspect ratio of the particles. The dimensions and arrangement of the cutting edges for both types of surface textures are also shown in Figure 7.2. These designs do not reflect a systematic statistical approach but rather a cumulative trial and error historical perspective. In the following sections, we will examine the various surface textural parameters. 
Type A:

Feature arrays

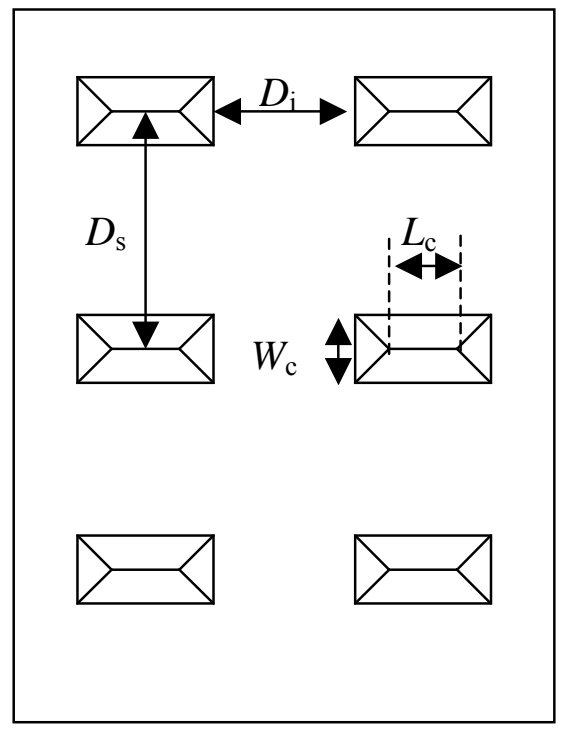

Type B:

Feature arrays with offset arrangement

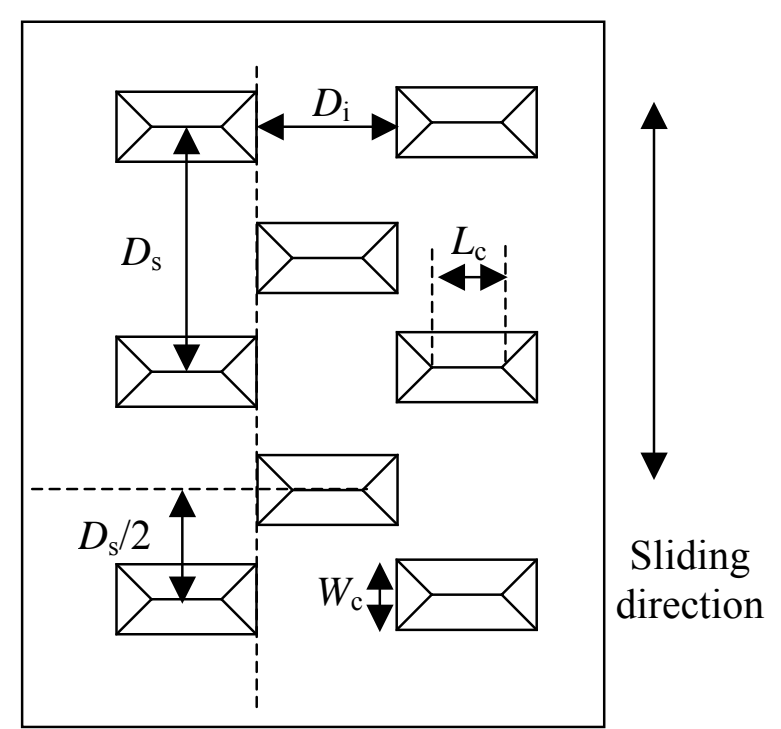

$L_{\mathrm{c}}$ : cutting-edge length

$W_{\mathrm{c}}$ : cutting-edge width

$H_{\mathrm{c}}$ : cutting-edge height

$D_{\mathrm{s}}$ : pitch in the sliding direction

$D_{\mathrm{i}}$ : distance between adjacent features

$\rho$ : density of surface features (no. features per unit area)

(Uncertainty of measurement of the dimensions come from the microfabrication process. A relative standard uncertainty is estimated to be less than 10\%)

\begin{tabular}{|l|c|c|c|c|c|c|c|}
\hline $\begin{array}{l}\text { Surface } \\
\text { texture }\end{array}$ & $\begin{array}{c}L_{\mathrm{c}} \\
(\mu \mathrm{m})\end{array}$ & $\begin{array}{c}W_{\mathrm{c}} \\
(\mu \mathrm{m})\end{array}$ & $\begin{array}{c}H_{\mathrm{c}} \\
(\mu \mathrm{m})\end{array}$ & $\begin{array}{c}D_{\mathrm{s}} \\
(\mu \mathrm{m})\end{array}$ & $\begin{array}{c}D_{\mathrm{i}} \\
(\mu \mathrm{m})\end{array}$ & $\begin{array}{c}\rho \\
1 /(\mu \mathrm{m})^{2}\end{array}$ & $\begin{array}{c}\text { Offset } \\
\text { features }\end{array}$ \\
\hline A-1 & 55.0 & 5.0 & 4.2 & 200 & 40 & 0.00005 & No \\
\hline A-2 & 15.0 & 5.0 & $3.4 ; 2.8$ & 200 & 80 & 0.00005 & No \\
\hline A-3 & 7.0 & 5.0 & $3.0 ; 2.2$ & 200 & 90 & 0.00005 & No \\
\hline A-4 & 6.8 & 5.0 & 2.3 & 10 & 10 & 0.005 & No \\
\hline A-5 & 3.2 & 2.0 & 0.8 & 4 & 4 & 0.3125 & No \\
\hline B-1 & 6.2 & 2.0 & $0.9 ; 1.3$ & 10 & 8 & 0.0125 & Yes \\
\hline B-2 & 3.5 & 2.0 & 1.4 & 20 & 4 & 0.0125 & Yes \\
\hline B-3 & 3.6 & 2.0 & 0.6 & 10 & 4 & 0.025 & Yes \\
\hline B-4 & 3.8 & 1.0 & 1.2 & 10 & 4 & 0.025 & Yes \\
\hline
\end{tabular}

Figure 7.2 A schematic representation of dimensions of the surface textures containing wedge-shaped cutting edges. 


\subsection{Results}

The surface textures were fabricated and used to generate UHMWPE particles in linear reciprocating wear tests under a nominal contact pressure of $3 \mathrm{MPa}$, a stroke length of $19 \mathrm{~mm}$, a frequency of $1.5 \mathrm{~Hz}$, and an average sliding speed of $57.2 \mathrm{~mm} / \mathrm{s}$.

The feature length is the most important parameter and is varied from $3.2 \mu \mathrm{m}$ to $55 \mu \mathrm{m}$. The feature height is varied from $0.8 \mu \mathrm{m}$ to $4.2 \mu \mathrm{m}$ and the pitch is varied from (4 to 200) $\mu \mathrm{m}$. The overall geometric design is reflected in group A and group B designs.

\subsubsection{Effect of the cutting-edge length}

To determine the effect of cutting-edge length, we designed the surface textures with different cutting-edge lengths with the same geometric parameters $D_{\mathrm{s}}$, $D_{\mathrm{i}}$, and density of surface features $\rho_{\mathrm{s}}$ (surface textures A-1, A-2, and A-3 in Figure 7.2). The micro-images of the surface texture, single cutting-edge feature, generated UHMWPE particles and particle distributions are shown in Figures 7.3-7.5. (Length \pm standard uncertainty) of cutting edges for surface textures A-1, A-2, and A-3 are $(55 \pm 5.1) \mu \mathrm{m},(15 \pm 1.1) \mu \mathrm{m}$, and $(7 \pm 0.5) \mu \mathrm{m}$ respectively. The number averaged mean particle-length generated from these three surface textures are $(43.1 \pm 7.1) \mu \mathrm{m}(78 \%$ of cutting edge length), (10.2 \pm 3.9$) \mu \mathrm{m}(68 \%)$, and $(6.2 \pm 2.1) \mu \mathrm{m}(89 \%)$. The median values of the particle lengths are also shown in the figures. A longer cutting-length leads to a larger particle-length. The results also show that the particle length is always smaller than the cutting-edge length about $68 \%$ to $89 \%$ of the cutting edge length. 
This may be due to particle attrition in the contact as well as viscoelastic effect at the two ends of the cutting edge. During the particle-generation process, the surfacefeature edge cuts into the UHMWPE material during the linear reciprocating wear process. Under the condition that the sliding direction is perpendicular to the cuttingedge length direction, the particle length should ideally be equal to the dimension of the cutting edge. Two factors may contribute to this observation. One is the viscoelastic behavior of UHMWPE. While the cutting edge penetrates into the material, the deformed length of the material is equal to the cutting-edge length. However, at two ends of the cutting edge, the viscoelastic behavior of the UHMWPE will stretch (similar to a rubber band) and eventually break. Because of the stretching, the final particle length will be shorter than the cutting edge length. Another possible explanation is the particles, once generated, can be cut again by the features before exiting the contact. 
Surface texture

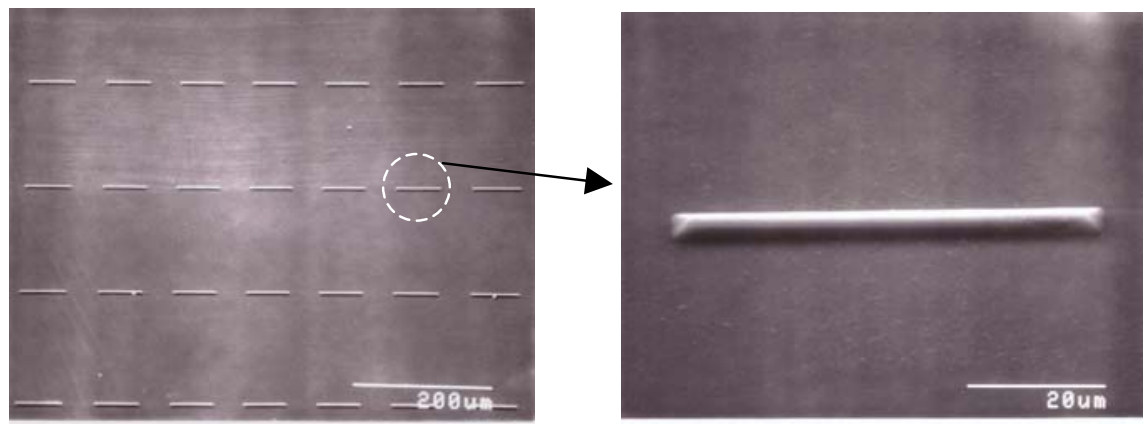

UHMWPE particles
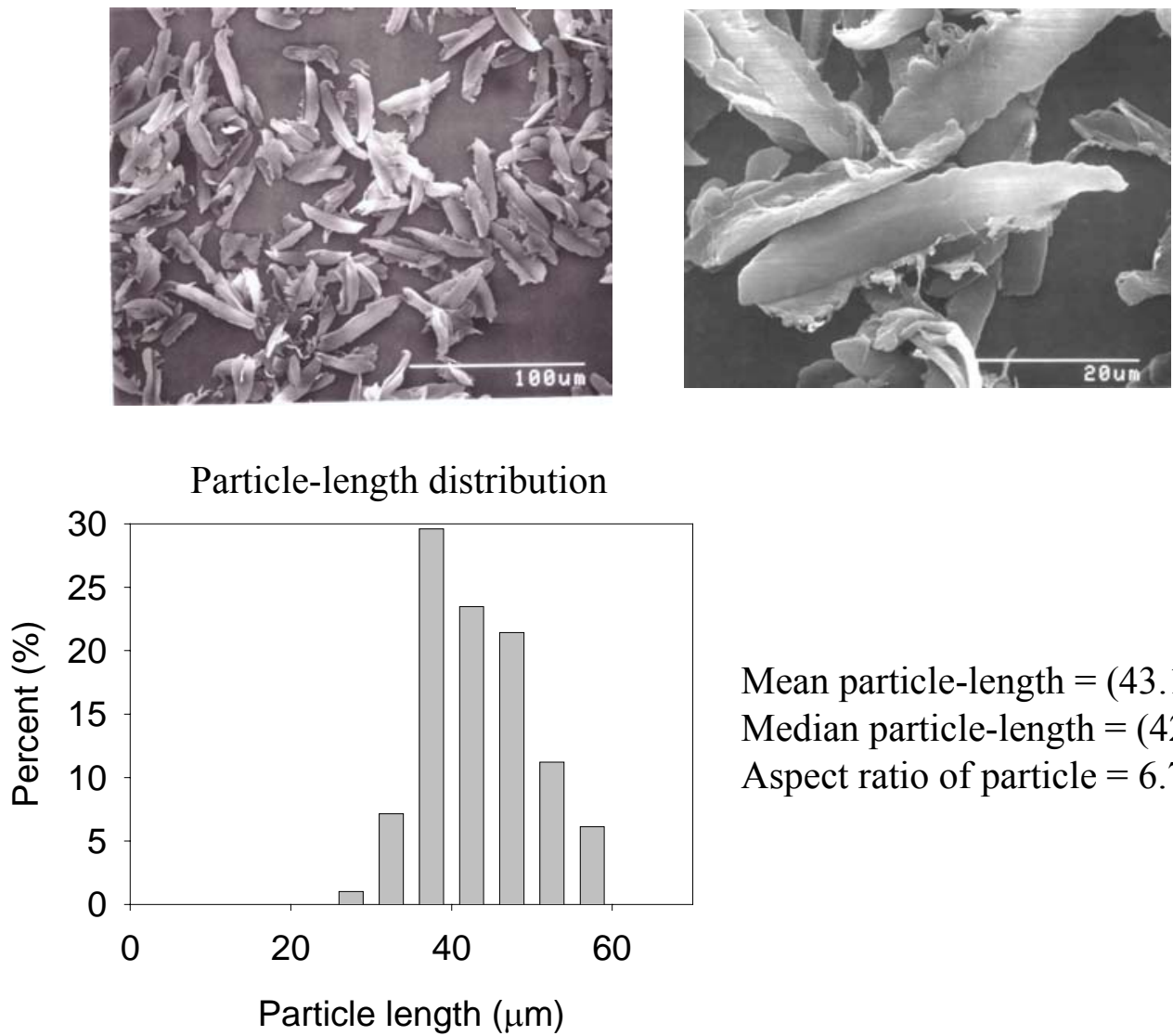

Mean particle-length $=(43.1 \pm 7.1) \mu \mathrm{m}$ Median particle-length $=(42.1 \pm 6.8) \mu \mathrm{m}$ Aspect ratio of particle $=6.7 \pm 1.1$

Figure 7.3 Micrographs of the surface texture A-1 $\left(L_{\mathrm{c}}=55 \mu \mathrm{m}, H_{\mathrm{c}}=4.2 \mu \mathrm{m}\right.$, $D_{\mathrm{s}}=200 \mu \mathrm{m}$ ) and generated UHMWPE particles (contact pressure $=3 \mathrm{MPa}$, average sliding speed $=57.2 \mathrm{~mm} / \mathrm{s}$ ) 


\section{Surface texture}

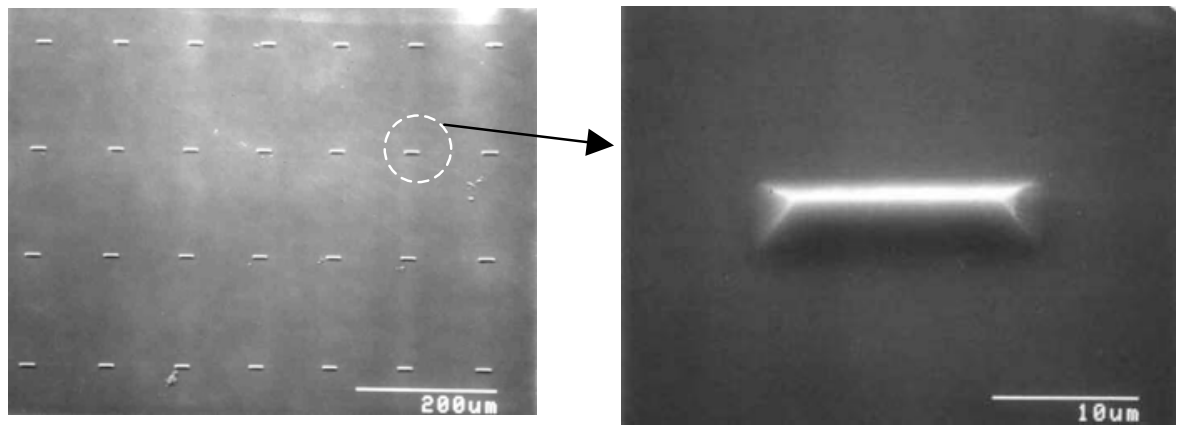

UHMWPE particles
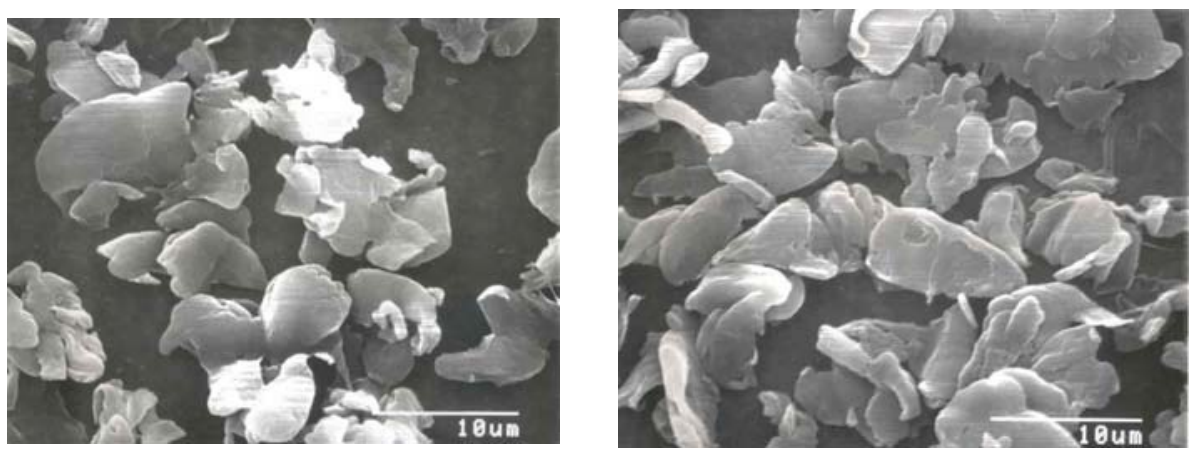

Particle-length distribution

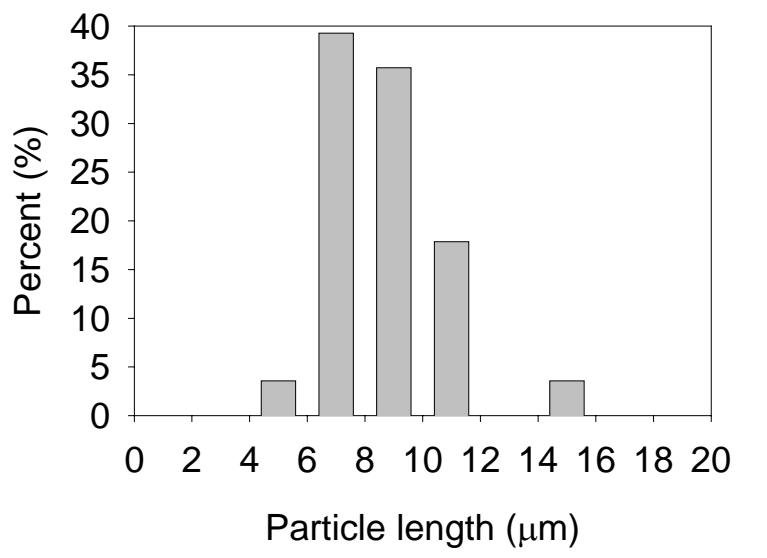

Mean particle-length $=(10.2 \pm 3.9) \mu \mathrm{m}$

Median particle-length $=(8.3 \pm 3.6) \mu \mathrm{m}$ Aspect ratio of particle $=2.2 \pm 0.7$

Figure 7.4 Micrographs of the surface texture A-2 $\left(L_{\mathrm{c}}=15.0 \mu \mathrm{m}, H_{\mathrm{c}}=3.4 \mu \mathrm{m}\right.$, $D_{\mathrm{s}}=200 \mu \mathrm{m}$ ) and generated UHMWPE particles (contact pressure $=3 \mathrm{MPa}$, average sliding speed $=57.2 \mathrm{~mm} / \mathrm{s}$ ) 
Surface texture
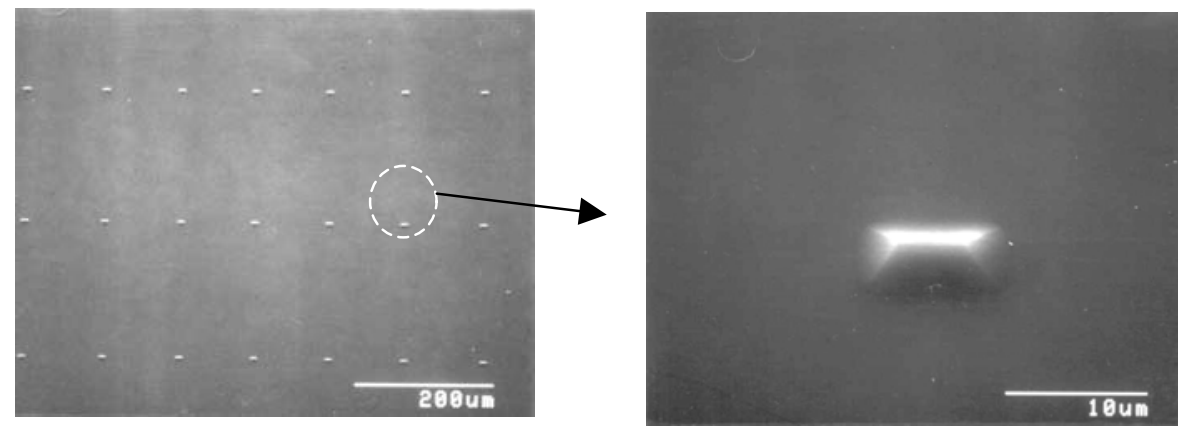

UHMWPE particles
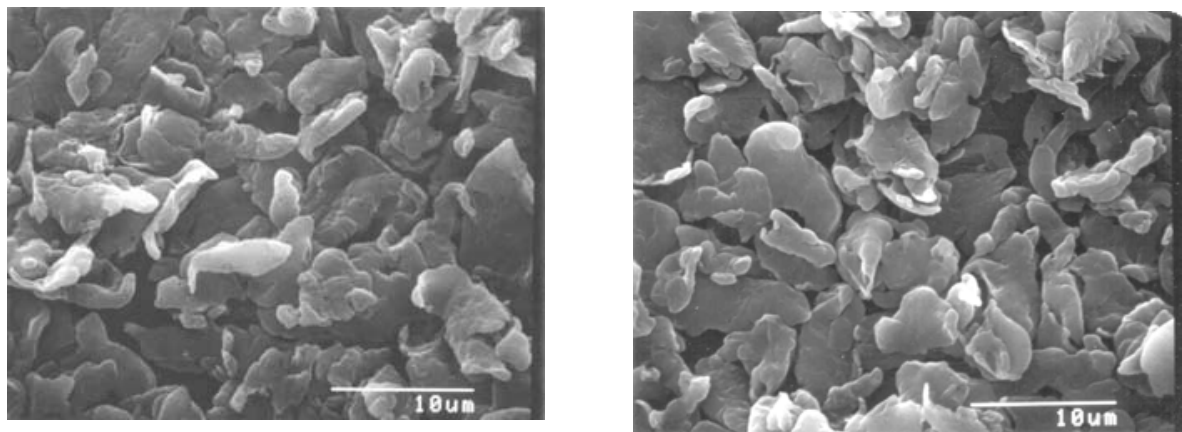

Particle-length distribution

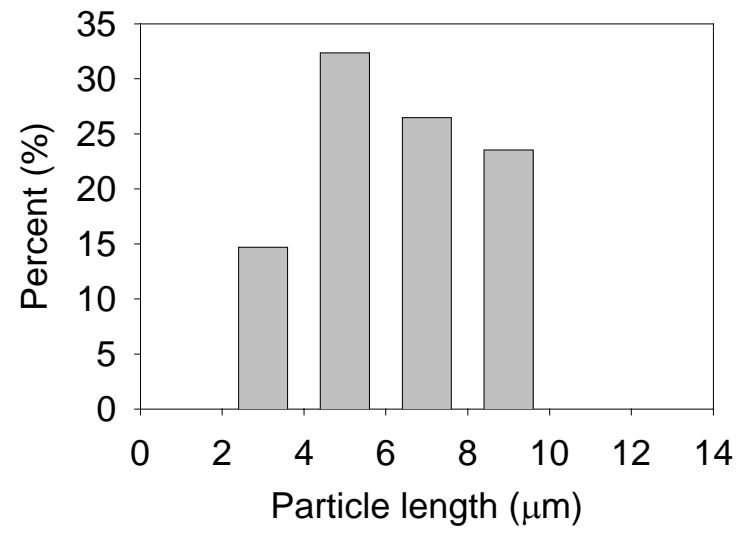

Mean particle-length $=(6.2 \pm 2.1) \mu \mathrm{m}$ Median particle-length $=(6.2 \pm 2.0) \mu \mathrm{m}$ Aspect ratio of particle $=2.6 \pm 0.6$

Figure 7.5 Micrographs of the surface texture A-3 $\left(L_{\mathrm{c}}=7 \mu \mathrm{m}, H_{\mathrm{c}}=3.0 \mu \mathrm{m}\right.$, $D_{\mathrm{s}}=200 \mu \mathrm{m}$ ) and generated UHMWPE particles (contact pressure $=3 \mathrm{MPa}$, average sliding speed $=57.2 \mathrm{~mm} / \mathrm{s}$ ) 


\subsubsection{Effect of the cutting-edge height}

To investigate the effects of cutting-edge height on the particle size and shape, cutting edges with different heights were microfabricated from the same pattern. Figure 7.6 compares the particles generated from surface pattern A-2 with different cutting-edge heights. The cutting-edge length is $15 \mu \mathrm{m}$ for both features and the cutting-edge heights are $2.8 \mu \mathrm{m}$ and $3.4 \mu \mathrm{m}$, respectively. The aspect ratios of particles are $3.1 \pm 0.7$ and $1.8 \pm 0.3$. The surface texture with smaller cutting edgeheights generated more elongated particles (larger aspect ratio of the particle), and the surface texture with a larger cutting-edge height generated particles with a smaller aspect ratio.

From a calculation of the load applied on each cutting edge, we estimate that the contact pressure is sufficient to push the cutting edges to the maximum penetration depths. Thus a larger cutting-edge height results in a larger penetration depth of the cutting edge. A deeper penetration of the cutting edge induces a larger volume of shear deformation of UHMWPE during the sliding process of the cutting edge. With the similar particle length, the larger shear deformation leads to a larger width and a smaller aspect ratio of the particle. This suggests that we can control the aspect ratio of the generated particles by adjusting the height of the cutting edge. 

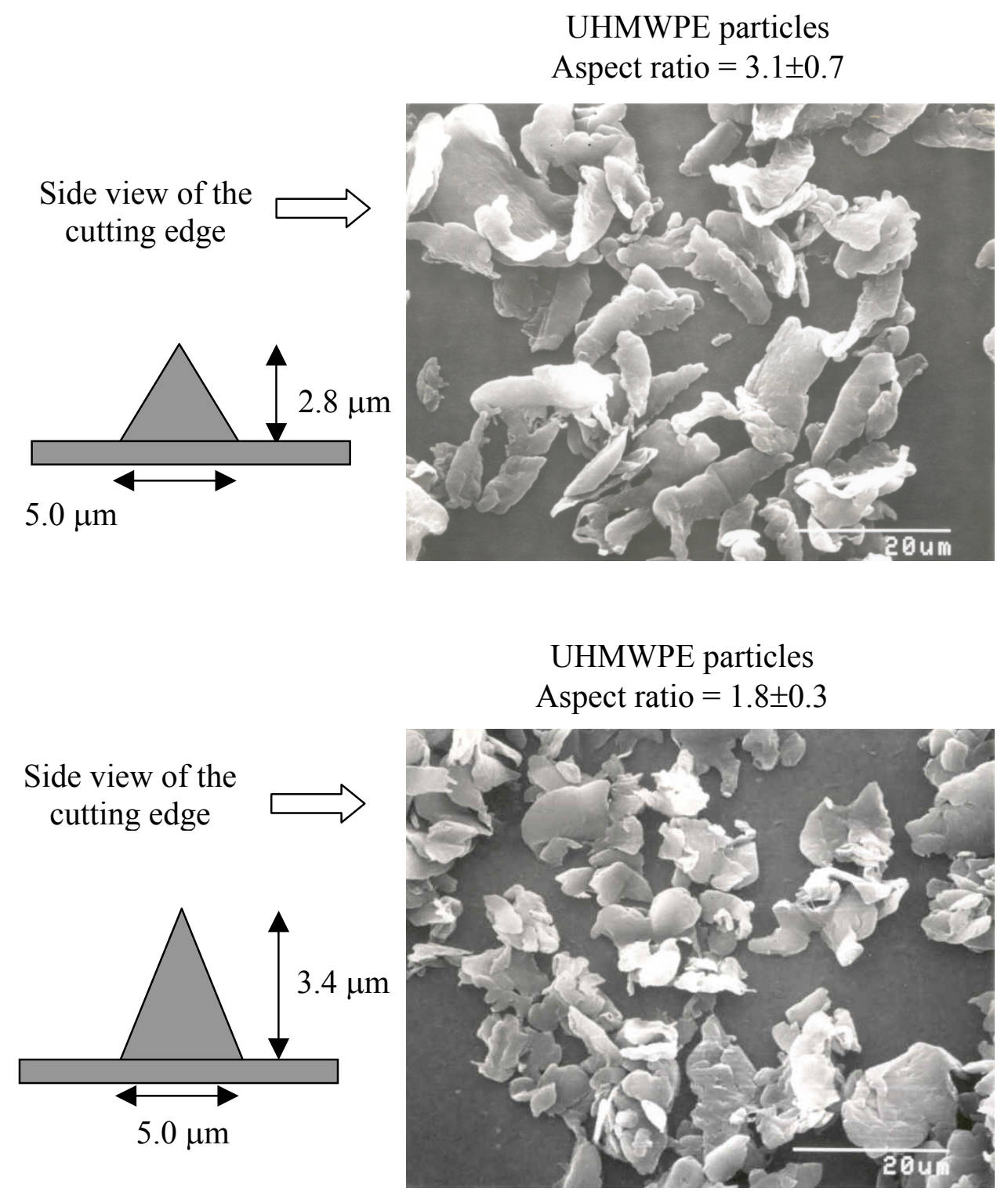

Figure 7.6 Comparison of UHMWPE particles generated from surface texture A-2 with different cutting-edge heights (contact pressure $=3 \mathrm{MPa}$, average sliding speed $=57.2 \mathrm{~mm} / \mathrm{s}$ ). 


\subsubsection{Effects of the cutting edge pattern}

To investigate the interaction between cutting edges along the UHMWPE pin sliding-direction, surface textures with different pitch $\left(D_{\mathrm{s}}\right)$ in the sliding direction and different distance between adjacent features $\left(D_{\mathrm{i}}\right)$ were applied in the particlegeneration process. When we reduced $D_{\mathrm{s}}$ of surface texture A-3 from $200 \mu \mathrm{m}$ to 10 $\mu \mathrm{m}$ (surface texture A-4), many irregular particles were generated (see Figure 7.7). It is said that the particles were generated from a non-effective micro-cutting process. A non-effective micro-cutting process was also observed for the particles generated from surface texture A-4 which has a $D_{\mathrm{s}}$ of $4 \mu \mathrm{m}$ as seen in Figure 7.8. When the pitch distance is too small in the sliding direction, overlapping of the cutting processes occurs by the interaction between adjacent cutting edges, and the individual cutting process cannot be completed. This is a two dimensional interference of the cutting process in the penetration and sliding directions. It ends up with the cutting edges wrapped in the UHMWPE. Then the repeated sliding process results in the generation of large and irregular particles without precise control. We suggest that there exists a minimum distance between the cutting features in the sliding direction in order to induce effective micro-cutting for generation of particles with controlled sizes and shapes. 
Surface texture
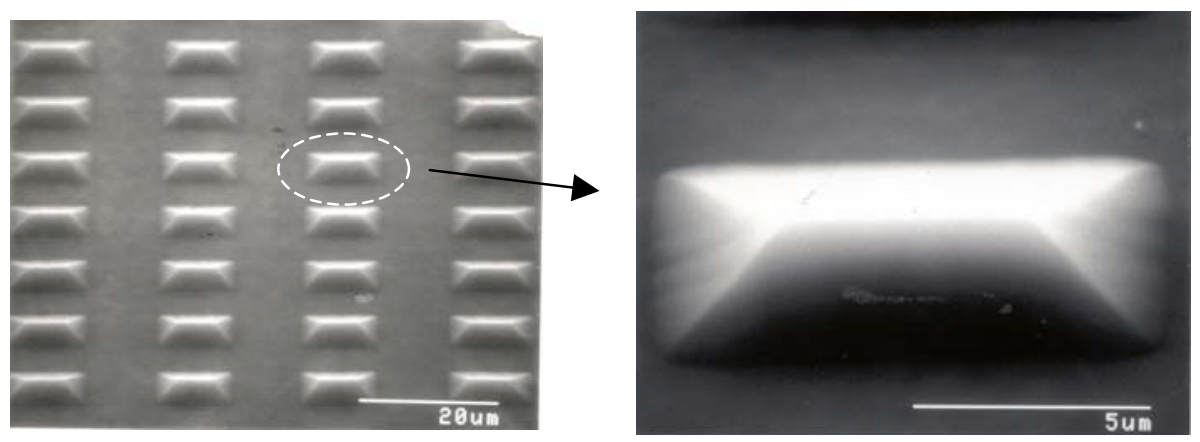

UHMWPE particles
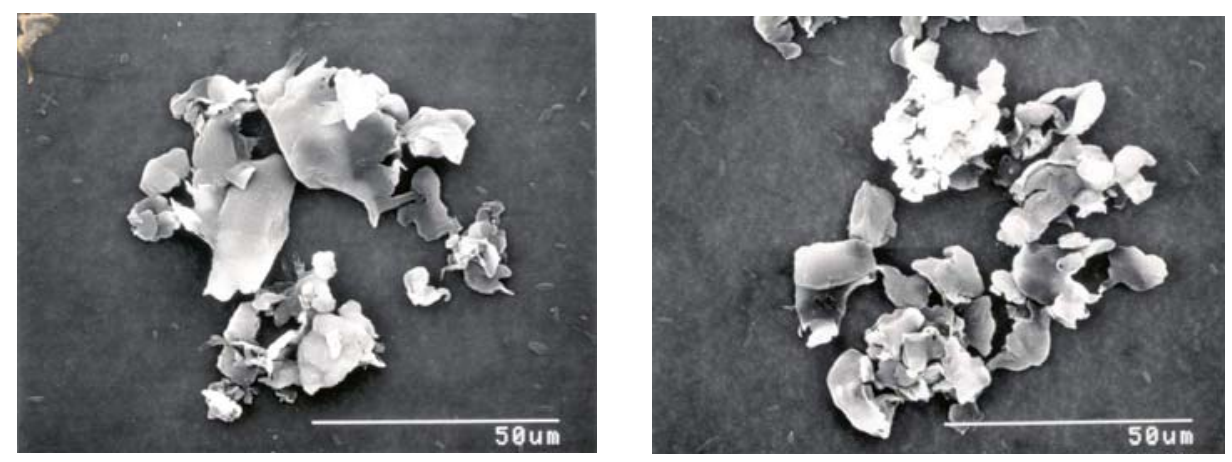

Particle length $\approx(5$ to 25$) \mu \mathrm{m}$

Many irregular particles were observed.

UHMWPE particles were generated without precisely controlled size and shape.

Figure 7.7 Micrographs of the surface texture A-4 $\left(L_{\mathrm{c}}=6.8 \mu \mathrm{m}, H_{\mathrm{c}}=2.3 \mu \mathrm{m}, D_{\mathrm{s}}=\right.$ $10 \mu \mathrm{m}$ ) and generated UHMWPE particles (contact pressure $=3 \mathrm{MPa}$, average sliding speed $=57.2 \mathrm{~mm} / \mathrm{s}$ ) 
Surface texture

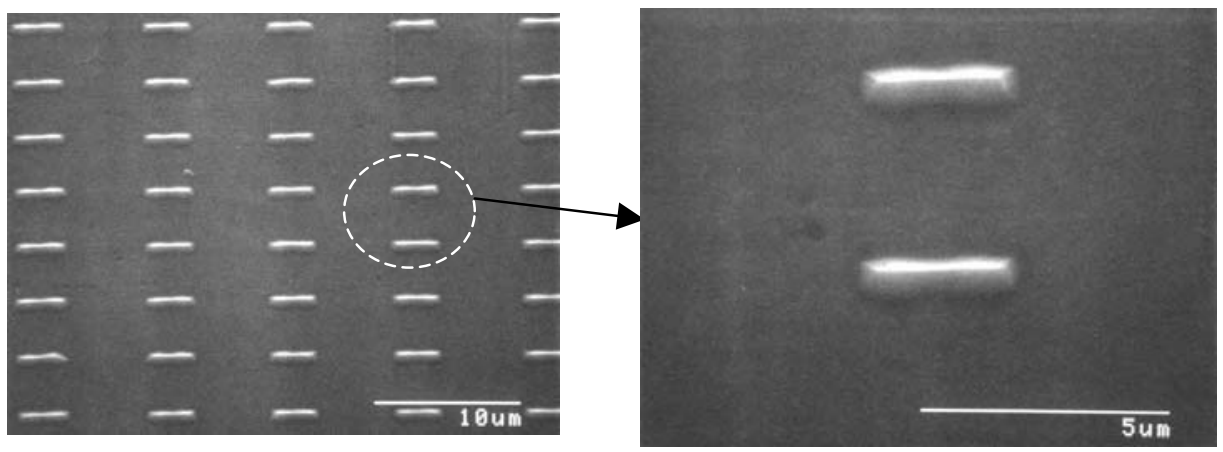

UHMWPE particles
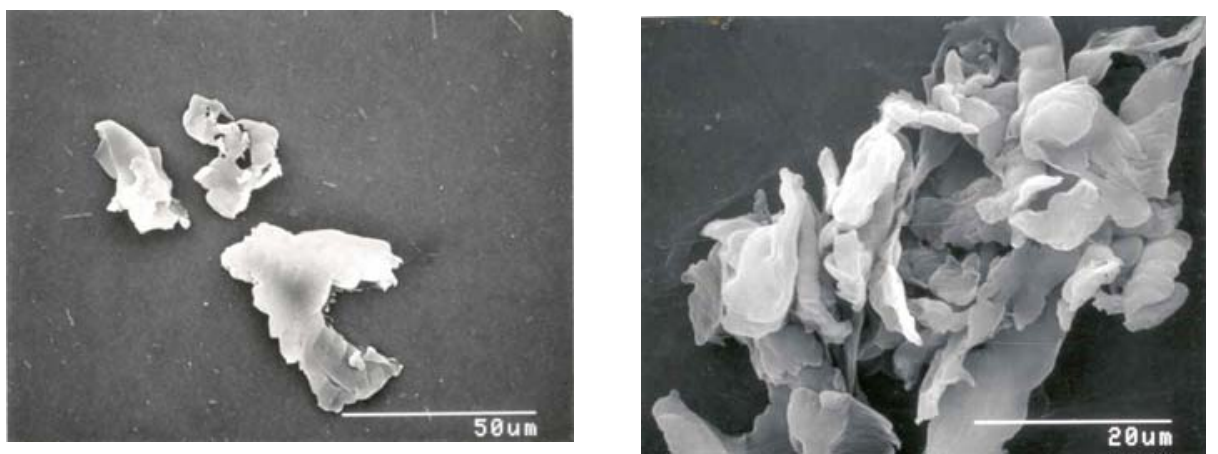

Particle length $\approx(10$ to 25$) \mu \mathrm{m}$

Many irregular particles were observed.

UHMWPE particles were generated without precisely controlled size and shape.

Figure 7.8 Micrographs of the surface texture A-5 $\left(L_{\mathrm{c}}=3.2 \mu \mathrm{m}, H_{\mathrm{c}}=0.8 \mu \mathrm{m}, D_{\mathrm{s}}=\right.$ $4 \mu \mathrm{m}$ ) and generated UHMWPE particles (contact pressure $=3 \mathrm{MPa}$, average sliding speed $=57.2 \mathrm{~mm} / \mathrm{s}$ ) 
Particles were also generated from the surface textures with offset arranged features (type B). Figures 7.9-7.10 show the images of the surface texture, single cutting-edge feature, and UHMWPE particles generated from surface textures B-1 and B-4. Large and irregular particles without size and shape control were generated. Much larger particles were observed compared to the particles shown in Figures 7.77.8 that were generated from the surface textures without offset features. It is shown that the offset arrangement of cutting edges further results in the interference of the cutting process in the direction perpendicular to the sliding direction. Materials between the adjacent cutting edges were involved in the non-effective cutting process. Thus the three dimensional interference of the micro-cutting process leads to much larger particles with indent marks on the surface.

We realize that a longer sliding distance arises from a larger penetration depth of the cutting edge. Thus a larger cutting-edge height may need a larger pitch to prevent the interaction between cutting edges. We calculated the ratio of $D_{\mathrm{s}}$ to $H_{\mathrm{c}}$ for each case and classify the surface textures into groups of clean micro-cutting and interference of micro-cutting as shown in Table 7.1. We found that the surface texture inducing an effective micro-cutting process has a $\left(D_{\mathrm{s}} / H_{\mathrm{c}}\right)$ value larger than 14 . However, when the value of $\left(D_{\mathrm{s}} / H_{\mathrm{c}}\right)$ is smaller than 9 , the interference of microcutting process was observed. To make sure that micro-cutting in the particlegeneration process is effective, we should design the surface texture to meet a minimum $\left(D_{\mathrm{s}} / H_{\mathrm{c}}\right)$ value of 14 . 
Surface texture
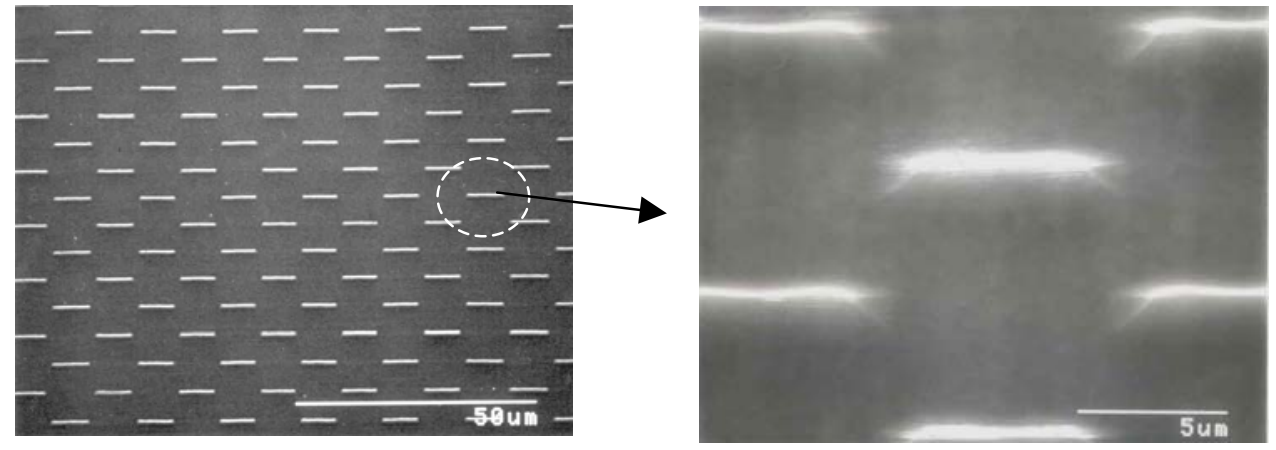

UHMWPE particles
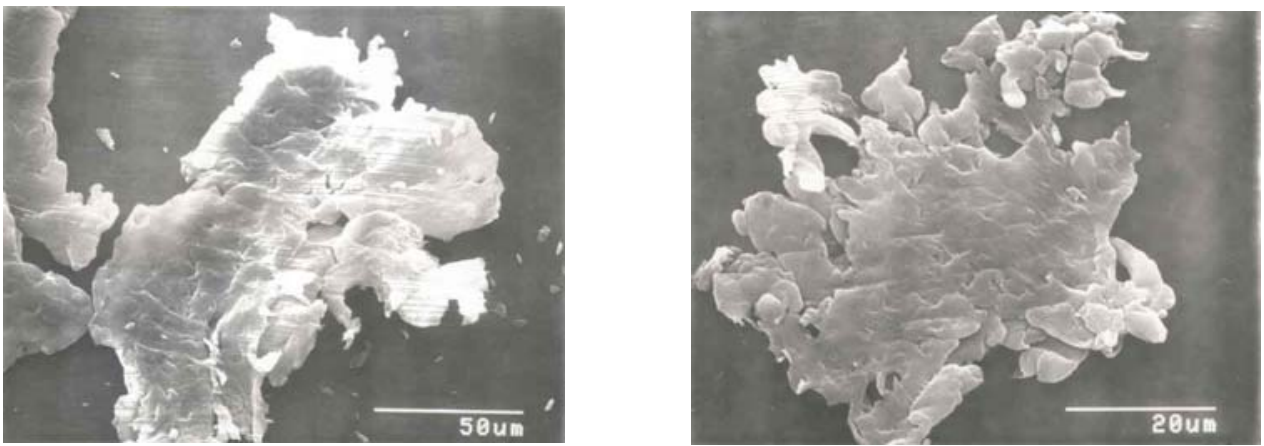

Particle length distribution

Many irregular and larger particles were observed. Indentation mark by the cutting edges were observed on the larger particles. UHMWPE particles were generated without precisely controlled size and shape.

Figure 7.9 Micrographs of the surface texture B-1 $\left(L_{\mathrm{c}}=6.2 \mu \mathrm{m}, H_{\mathrm{c}}=1.3 \mu \mathrm{m}, D_{\mathrm{s}}=\right.$ $10 \mu \mathrm{m}$ ) and generated UHMWPE particles (contact pressure $=3 \mathrm{MPa}$, average sliding speed $=57.2 \mathrm{~mm} / \mathrm{s}$ ) 


\section{Surface texture}
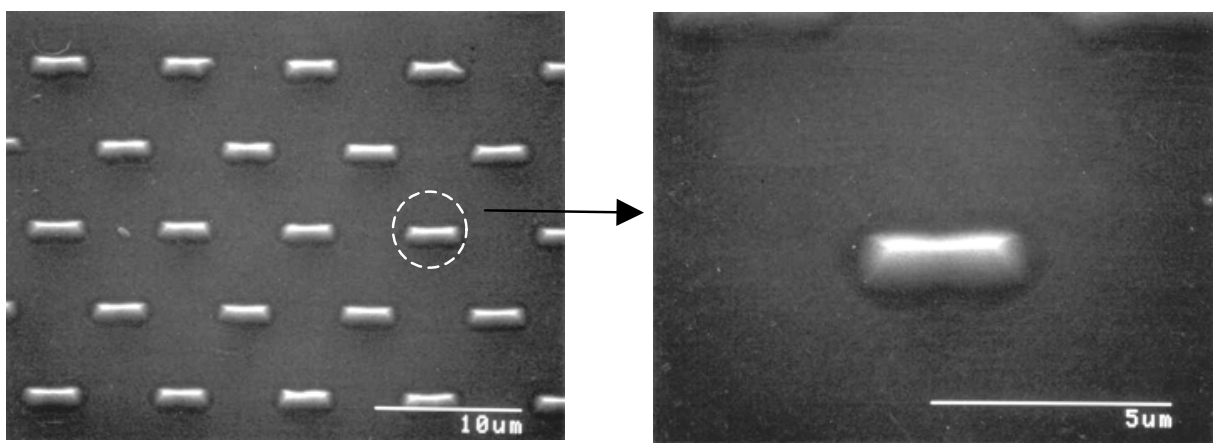

UHMWPE particles
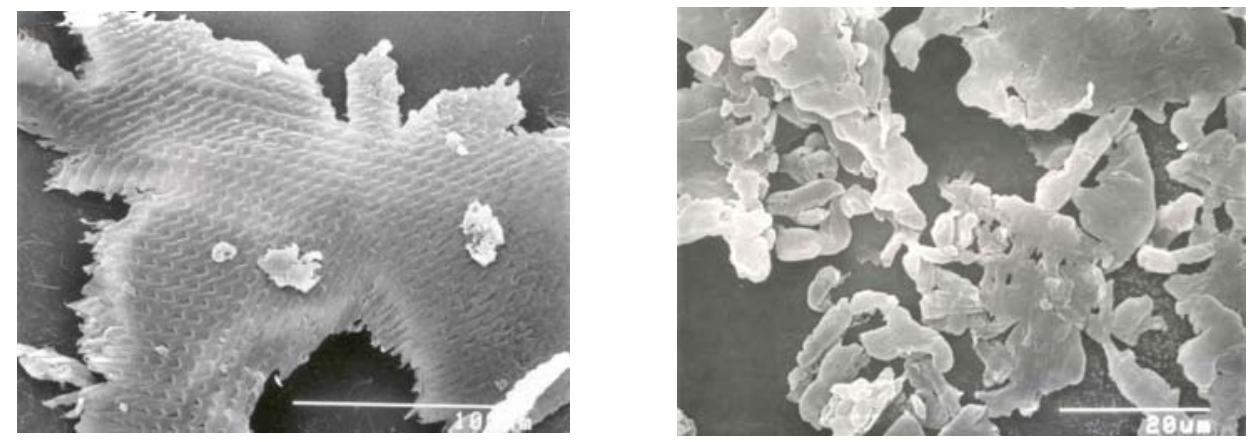

Many irregular and larger particles were observed. Indentation mark by the cutting edges were observed on the larger particles. UHMWPE particles were generated without precisely controlled size and shape.

Figure 7.10 Micrographs of the surface texture B-4 $\left(L_{\mathrm{c}}=3.8 \mu \mathrm{m}, H_{\mathrm{c}}=1.2 \mu \mathrm{m}, D_{\mathrm{s}}=\right.$ $10 \mu \mathrm{m}$ ) and generated UHMWPE particles (contact pressure $=3 \mathrm{MPa}$, average sliding speed $=57.2 \mathrm{~mm} / \mathrm{s}$ ) 
Table 7.1 Value of $\left(D_{\mathrm{s}} / H_{\mathrm{c}}\right)$ for effective and non-effective micro-cutting processes.

\begin{tabular}{|l|c|c|c|}
\hline Surface texture & $D_{\mathrm{s}} / H_{\mathrm{c}}$ & $\rho_{\mathrm{s}}\left[1 /(\mu \mathrm{m})^{2}\right]$ & Micro-cutting process \\
\hline A-1 & $47.6 \pm 2.0$ & 0.00005 & Clean cut \\
\hline A-2 & $58.8 \pm 2.2$ & 0.00005 & Clean cut \\
\hline A-3 & $66.7 \pm 2.5$ & 0.00005 & Clean cut \\
\hline B-2 & $14.3 \pm 0.4$ & 0.0125 & Clean cut \\
\hline B-3 & $16.7 \pm 0.6$ & 0.0125 & Clean cut \\
\hline A-4 & $4.3 \pm 0.3$ & 0.005 & 2D interference \\
\hline A-5 & $5.0 \pm 0.2$ & 0.3125 & 2D interference \\
\hline B-1 & $7.7 \pm 0.2$ & 0.125 & 3D interference \\
\hline B-4 & $8.3 \pm 0.3$ & 0.025 & 3D interference \\
\hline
\end{tabular}

The density of features $\left(\rho_{\mathrm{s}}\right)$ for each surface texture is also listed in Table 7.1. The surface texture with a smaller pitch has a larger density of features. The surface textures with offset arranged features also have larger values of the density of features. It is obvious that a smaller density of features can prevent the interaction between cutting edges on the surface. However, the experimental results did not show that the density of features was a critical parameter for effective micro-cutting process. On the other hand, the UHMWPE surface has a higher possibility to rub against the flat surface area for a smaller density of features. Adhesive wear may occur and tiny particles (submicrometer or smaller) without size and shape control may be generated. One should also optimize the density of features to induce an effective micro-cutting process and to avoid the particles generated from the non cutting-edge area. 


\subsubsection{Effect of pitch distance}

Figures 7.11 and 7.12 compare the UHMWPE particles generated from surface textures with $D_{\mathrm{s}}=20 \mu \mathrm{m}$ and $10 \mu \mathrm{m}$. The dimensions of the cutting edges for both surface textures are similar. It is seen that the surface texture with $D_{\mathrm{s}}=10 \mu \mathrm{m}$ leads to an average aspect ratio of the particles equal to $1.6 \pm 0.3$, and the surface texture with $D_{\mathrm{s}}=20 \mu \mathrm{m}$ leads to an average aspect ratio of particles equal to $1.9 \pm 0.5$. It indicates that a shorter $D_{\mathrm{s}}$ results in a larger width of the generated particles. With a shorter distance between cutting edges, the interaction between the two features in the sliding direction is stronger. The adjacent cutting-edge along the sliding direction induces a constraint of the flow of the shear deformation during the sliding process. A shorter distance between features $\left(D_{\mathrm{s}}\right)$ results in a larger shear volume by the cutting-edge sliding process. Thus a larger volume of material is deformed and it leads to a larger particle width (with the same particle length, it means a smaller aspect ratio). 
Surface texture

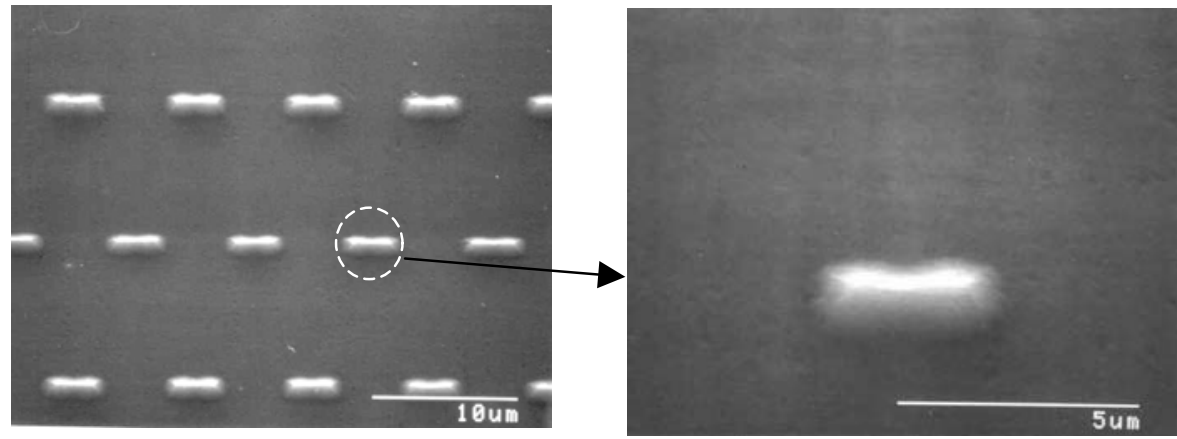

UHMWPE particles
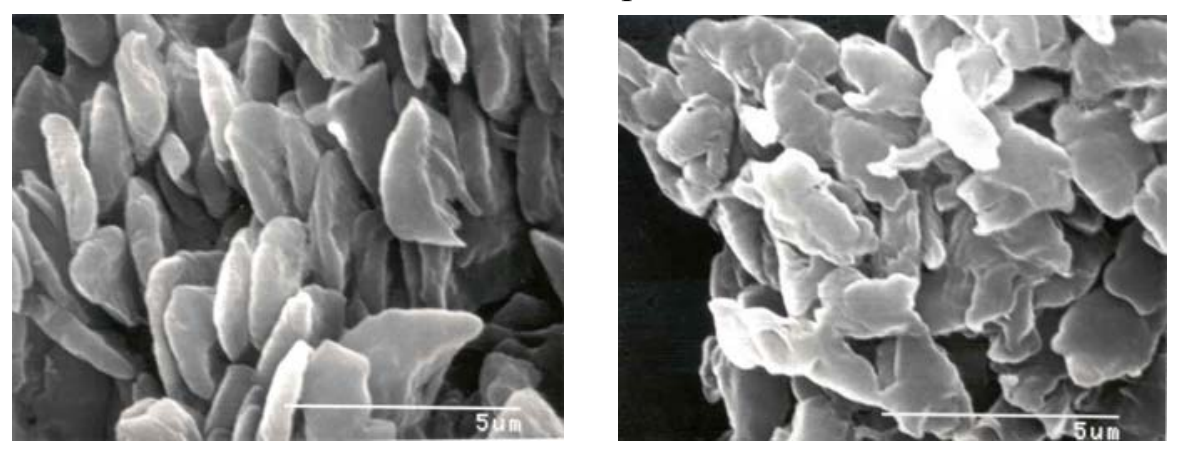

Particle-length distribution

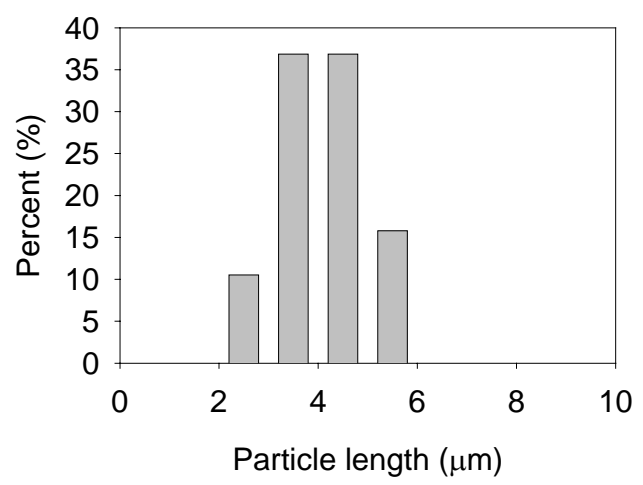

Mean particle-length $=(4.2 \pm 0.8) \mu \mathrm{m}$

Median particle-length $=(4.1 \pm 0.8) \mu \mathrm{m}$

Aspect ratio of particle $=1.9 \pm 0.5$

Figure 7.11 Micrographs of the surface texture B-2 $\left(L_{\mathrm{c}}=3.5 \mu \mathrm{m}, H_{\mathrm{c}}=1.4 \mu \mathrm{m}, D_{\mathrm{s}}=\right.$ $20 \mu \mathrm{m}$ ) and generated UHMWPE particles (contact pressure $=3 \mathrm{MPa}$, average sliding speed $=57.2 \mathrm{~mm} / \mathrm{s}$ ) 
Surface texture

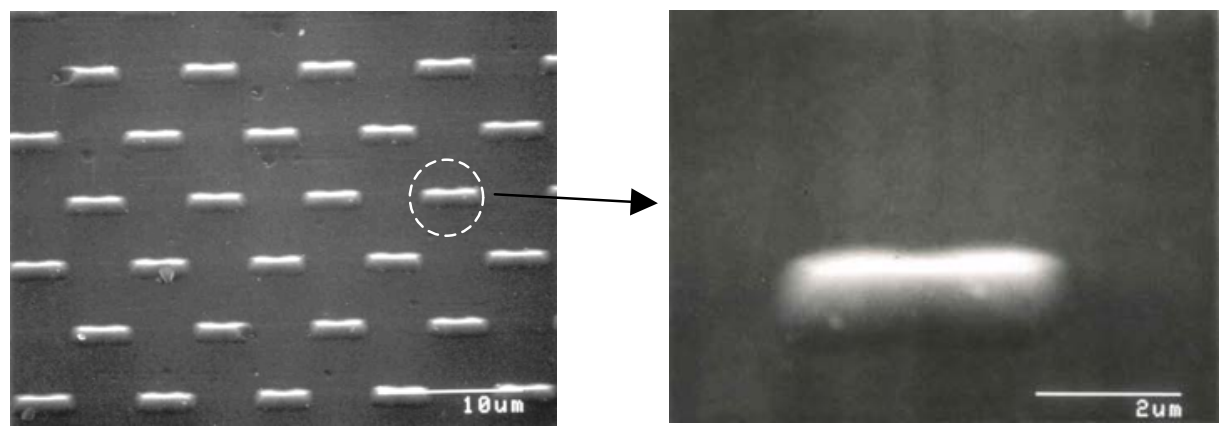

UHMWPE particles
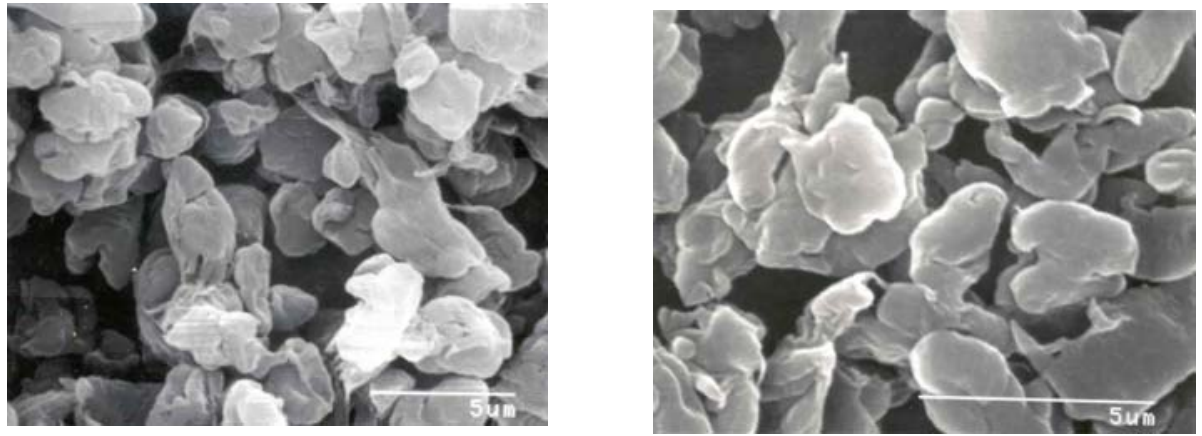

Particle-length distribution

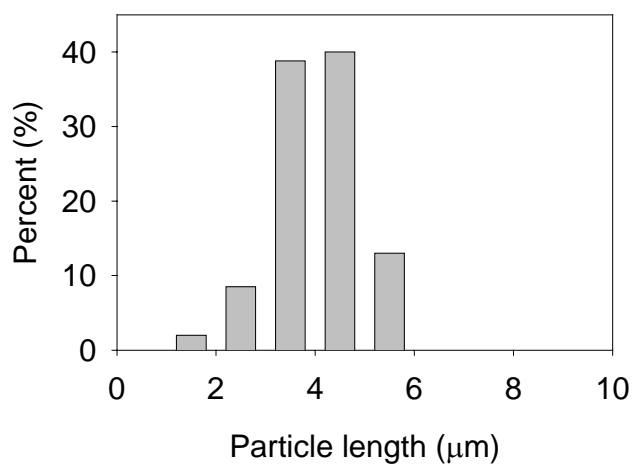

Mean particle-length $(3.9 \pm 0.7) \mu \mathrm{m}$

Median particle-length $=(4.1 \pm 0.8) \mu \mathrm{m}$

Aspect ratio of particle $=1.6 \pm 0.3$

Figure 7.12 Micrographs of the surface texture B-3 $\left(L_{\mathrm{c}}=3.6 \mu \mathrm{m}, H_{\mathrm{c}}=0.6 \mu \mathrm{m}, D_{\mathrm{s}}=\right.$ $10 \mu \mathrm{m}$ ) and generated UHMWPE particles (contact pressure $=3 \mathrm{MPa}$, average sliding speed $=57.2 \mathrm{~mm} / \mathrm{s}$ ) 


\subsubsection{Effects of mechanical conditions}

The effect of the load on the dimensions of the generated particle was studied by changing the normal load applied on the same surface texture. Figure 7.13 shows a comparison of UHMWPE particles generated form the surface texture A-3 with a 7 $\mu \mathrm{m}$ cutting-edge length (shown in Figure 7.5) under different normal loads. The wear tests were run under a $1.5 \mathrm{~Hz}$ frequency with an average speed of $57.2 \mathrm{~mm} / \mathrm{s}$. The average particle width is $10.1 \mu \mathrm{m}$ under a normal load of $192 \mathrm{~N}$ (6 MPa contact pressure) compared to an average particle width of $6.2 \mu \mathrm{m}$ under a normal load of 96 $\mathrm{N}$ (3 MPa contact pressure). A larger normal load leads to a larger particle width and thus a smaller aspect ratio when the same surface texture is used.

Increase of normal load yields a larger penetration depth of the cutting edge. While the applied normal load is sufficient to push the cutting edge all the way into UHMWPE, an increase of the load will not make the cutting edge penetrate deeper. The excess load is acting as the resistant force on the cutting edges during the cutting process. Thus more material deformation occurs in order to accumulate sufficient resistant force to push away the cutting edge and complete a micro-cutting process. 
Load $=192 \mathrm{~N}$

Aspect ratio of particle $=1.9 \pm 0.5$

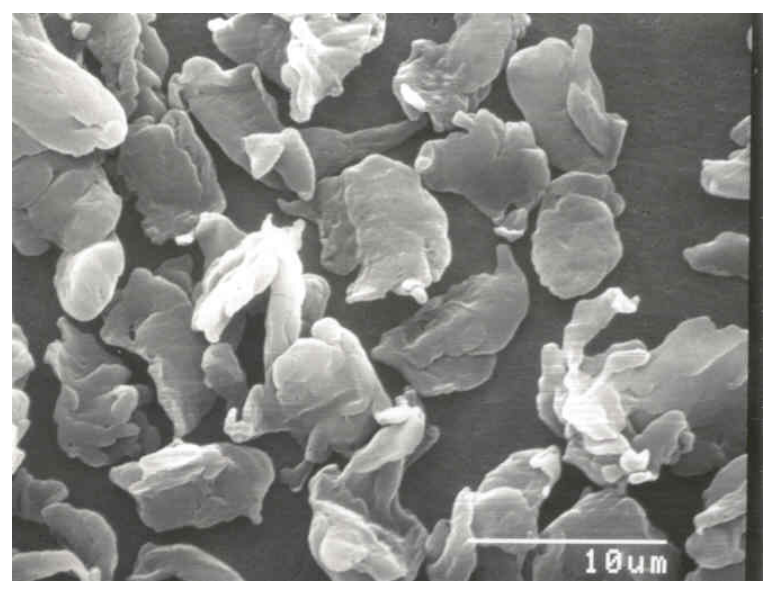

Load $=96 \mathrm{~N}$

Aspect ratio of particle $=2.6 \pm 0.6$

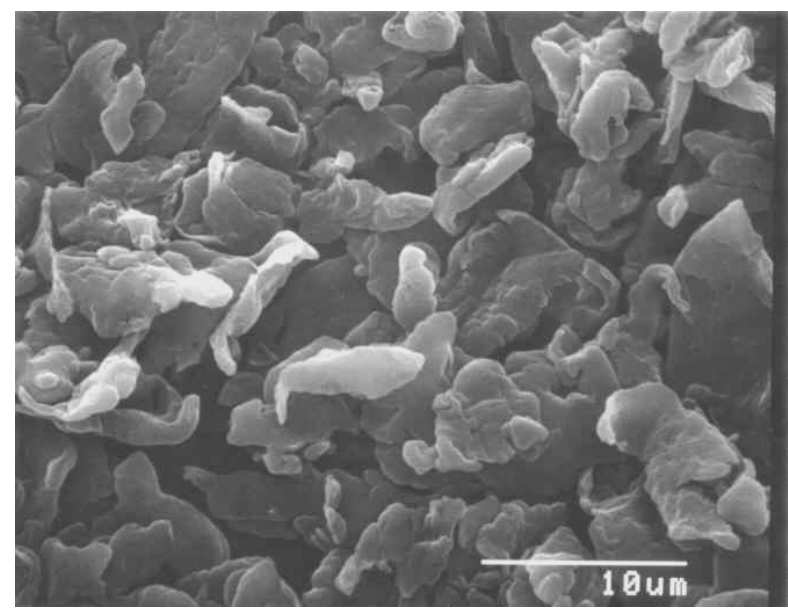

Figure 7.13 Comparison of UHMWPE particles generated under different normal loads (by using the surface texture A-3 with cutting edge length $=7 \mu \mathrm{m}$, average sliding speed $=57.2 \mathrm{~mm} / \mathrm{s}$ ). 
Effect of the sliding speed on the dimensions of the generated particle was studied for the surface texture A-1. Figure 7.14 shows a comparison of UHMWPE particles generated under a normal load of $96 \mathrm{~N}$ (3 MPa contact pressure) and with different sliding speeds. The results show that a higher sliding speed $(57.2 \mathrm{~mm} / \mathrm{s})$ yields more elongated particles (larger aspect ratio of the particle) and a lower sliding speed $(27.6 \mathrm{~mm} / \mathrm{s})$ results in particles with a smaller aspect ratio.

The sliding speed influences the residence time of the cutting edge during the cutting process, especially for a viscoelastic material. During the sliding process of the cutting edge, a lower sliding speed allows more stress relaxation while the material resistant force is increasing. It leads to a longer residence time to develop sufficient stress to break material and form a particle. Thus a lower speed leads to a larger particle width and a smaller aspect ratio of the particle. 
Average sliding speed $=27.6 \mathrm{~mm} / \mathrm{s}$

Aspect ratio of particle $=4.3 \pm 0.9$

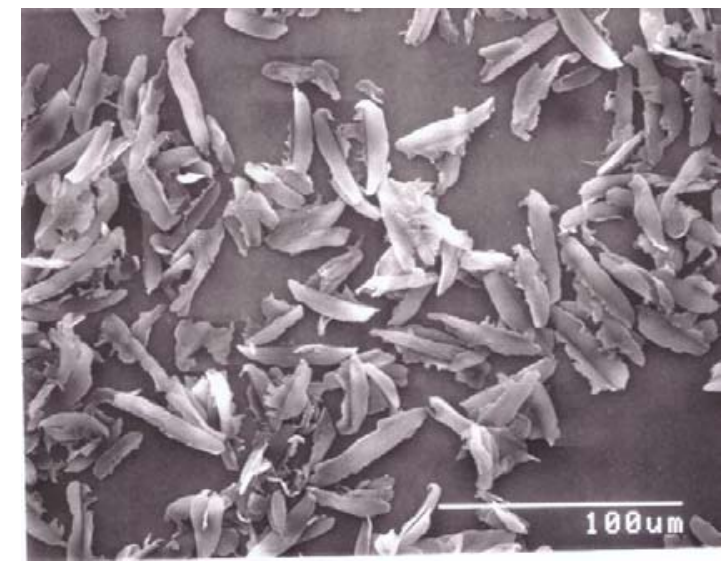

Average sliding speed $=57.2 \mathrm{~mm} / \mathrm{s}$

Aspect ratio of particle $=6.7 \pm 1.1$

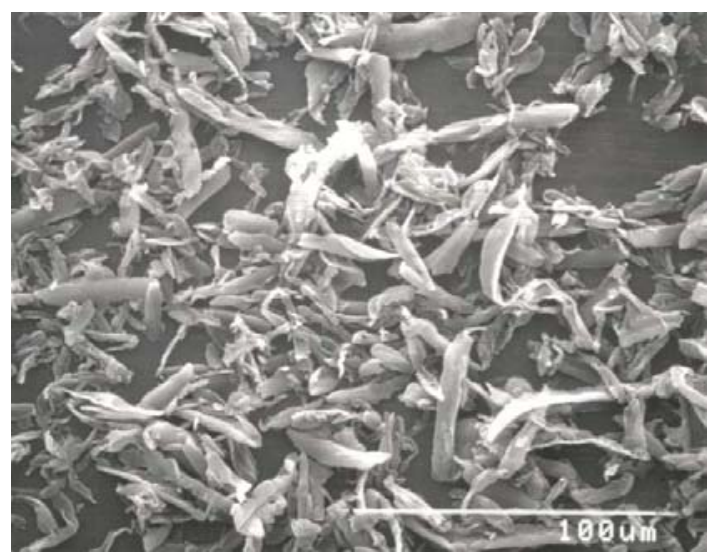

Figure 7.14 Comparison of micrographs of UHMWPE particles generated by the surface texture A-1 under $3 \mathrm{MPa}$ contact pressure and sliding speeds of $27.6 \mathrm{~mm} / \mathrm{s}$ and $57.2 \mathrm{~mm} / \mathrm{s}$ respectively. 


\subsection{Discussion}

\subsubsection{Effect of surface-texture uniformity on particle distribution}

Figure 6.8 in Chapter 6 showed the distribution of the lengths of the particles from surface textures made with $\mathrm{SiC}$ abrasive paper. Obviously, the particle-lengthdistribution curve is shifted to the right (larger particle-length) when the larger particles are produced by rougher surface textures. A clear difference of the average particle size in the two particle populations is observed in Figure 6.8. However, if we look closely at the particle distribution, the particle length ranges actually from several micrometers up to $100 \mu \mathrm{m}$ for both cases. Although it is narrower than the distribution of commercial UHWMPE particles, the particle-length distribution is still sufficiently broad so that some overlap between particle populations is inevitable.

The problem of surface-texture uniformity was overcome by applying microfabrication techniques to prepare surface features with precisely controlled dimensions described in Chapter 7. The uniformity of the surface texture is improved by precisely controlling the dimension of each feature of the surface texture.

We compare the particle populations with similar mean particle-length with two different kinds of surface textures. Particles generated from the microfabricated Si surface texture A-1 (mean particle length $43.1 \mu \mathrm{m}$ ) are compared with the particles generated from $\mathrm{SiC}$ abrasive paper with similar mean particle length (mean particle length $42.1 \mu \mathrm{m}$ ) in Figure 7.15. With similar mean particle length, $60 \%$ of the particles are in the range from $37 \mu \mathrm{m}$ to $46 \mu \mathrm{m}$. Also the entire particle distribution range has been reduced from $100 \mu \mathrm{m}$ to $35 \mu \mathrm{m}$, and the $95 \%$ confidence level from 
$3.0 \mu \mathrm{m}$ to $1.43 \mu \mathrm{m}$. Hence, we have demonstrated that by using a more uniform surface texture, a narrower particle distribution is obtained.

Particles generated from $\mathrm{SiC}$ abrasive paper made texture

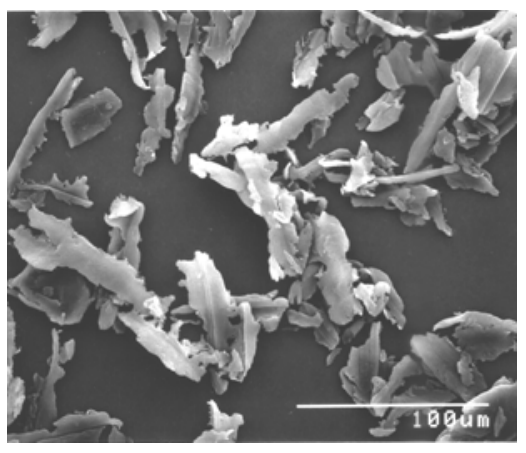

Particles generated from microfabricated surface texture

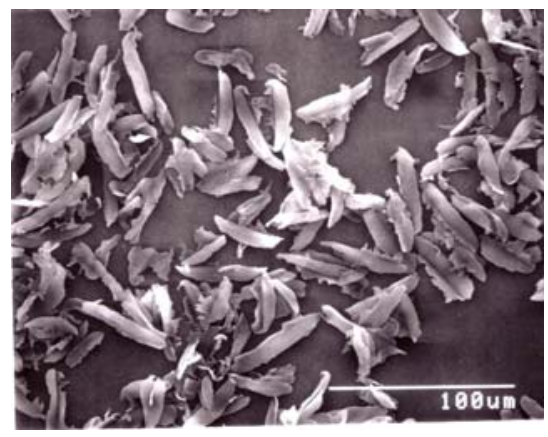

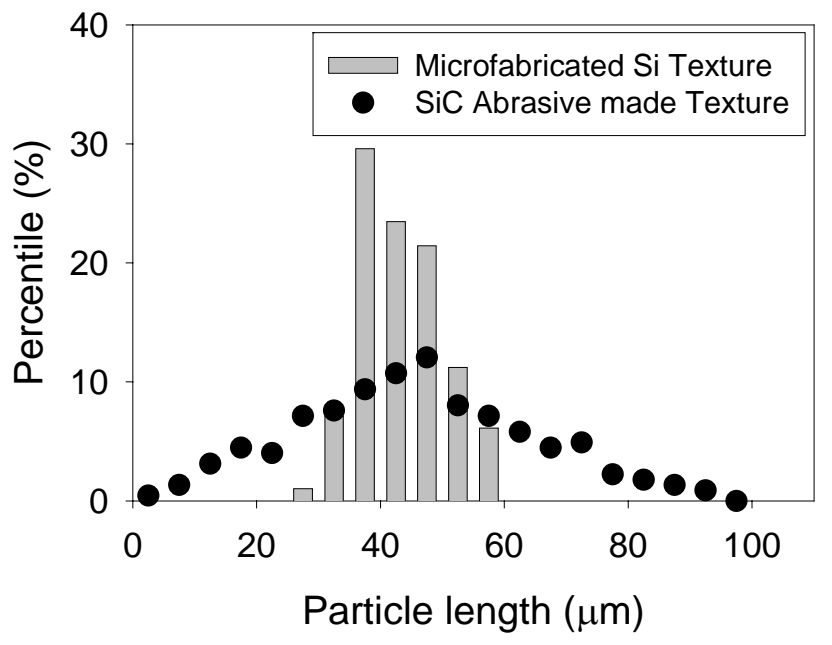

(Uncertainty of the measurement comes from the image measurement of particles which is estimated to be less than a relative standard uncertainty of 5\%)

Figure 7.15 Comparison of particle length distribution between particles generated from a $\mathrm{SiC}$ abrasive paper made surface texture and a microfabricated surface texture. 


\subsubsection{Significance for bioactivity tests}

Different size ranges of UHWMPE wear particles are needed for bioactivity studies. When a particle is small enough to be engulfed by a macrophage cell, it is said that the particle is phagocytosable. For a further analysis of the effects of phagocytosis of wear particles on sequential immunological reactions, particle populations within phagocytosable and non-phagocytosable regimes are needed for bioactivity tests. The size of a single macrophage cell is about $10 \mu \mathrm{m}$. It is recognized that a particle size larger than $10 \mu \mathrm{m}$ is difficult for a macrophage cell to engulf. The particle populations described in this chapter represent one non-phagocytosable particle population (mean particle length $43.1 \mu \mathrm{m}$ ), two phagocytosable particle populations (mean particle length $6.2 \mu \mathrm{m}, 4.2 \mu \mathrm{m}$ ), and a particle population (mean particle length $10.2 \mu \mathrm{m}$ ), with a particle length close to the macrophage-cell size. This represents a sufficient coverage of particles for investigating the macrophage-phagocytosis effect on particleinduced biological responses. The particle distributions of one non-phagocytosable particle population (mean particle length $43.1 \mu \mathrm{m}$ ) and one phagocytosable particle population (mean particle length $6.2 \mu \mathrm{m}$ ) are compared in Figure 7.16. Both particle populations have very narrow particle-length distributions without overlap. The particles can be used for biological response studies to elucidate the basic bioactivity mechanisms independently and systematically. 

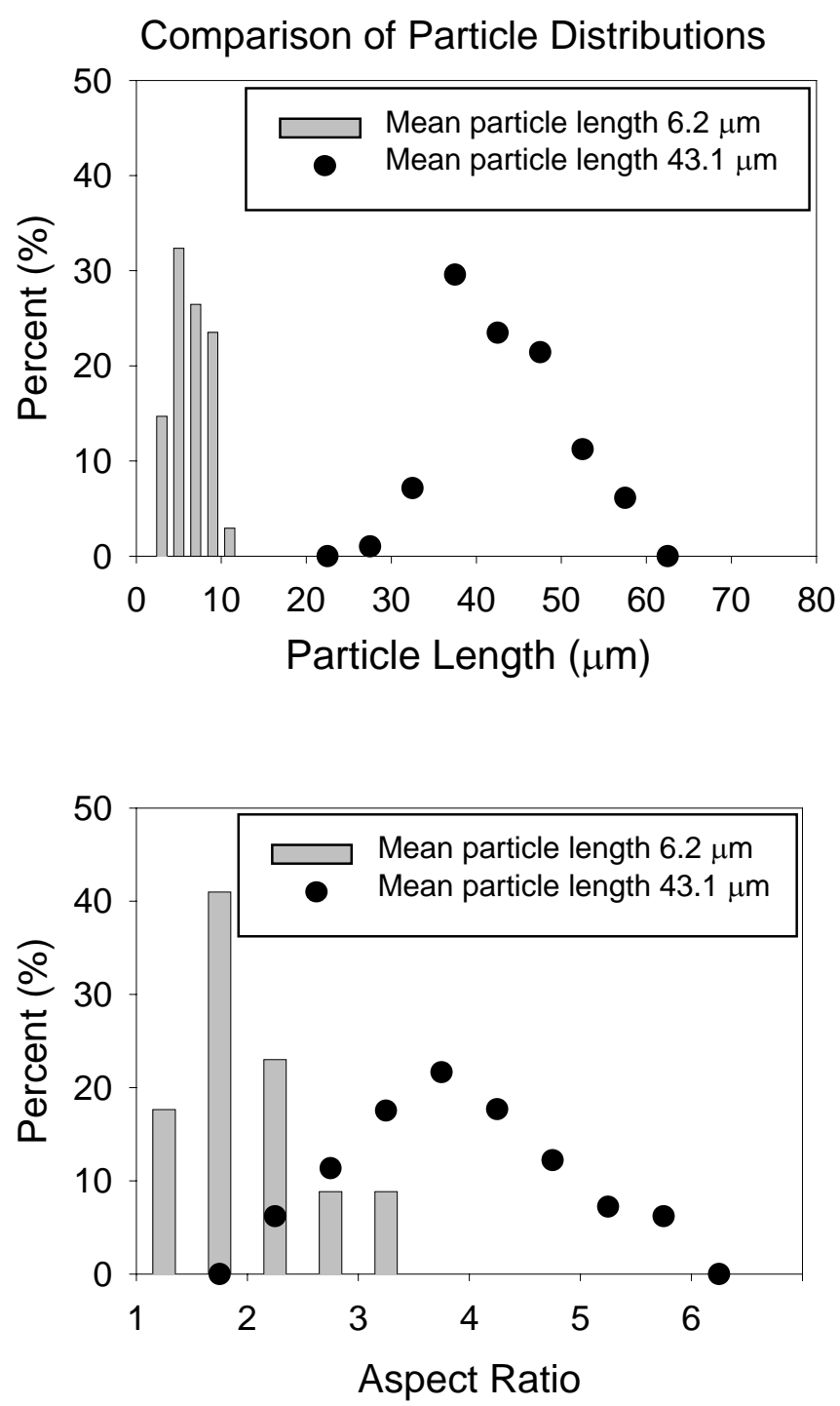

(Uncertainty of the measurement comes from the image measurement of particles which is estimated to be less than 5\%)

Figure 7.16 Comparison of two particle populations with distinct particle-length distribution. 


\subsection{Conclusions}

In this chapter, the basis of the surface-texture design for particle generation has been illustrated. The effects of cutting-edge dimensions, arrangement of surface features and mechanical conditions on the particle size and shape were evaluated. Surface textures of different kinds have been microfabricated for the generation of UHMWPE particles. The results showed that the cutting-edge length controls the particle length. The cutting-edge height is related to the aspect ratio of the particles. Critical values of $\left(D_{\mathrm{s}} / H_{\mathrm{c}}\right)$ were identified for effective micro-cutting. We also found that the applied load and the sliding speed have a strong influence on the sliding process of the cutting edges and have influence on the width and aspect ratio of a particle.

However, the exact mechanisms for the above phenomena are not evident. To achieve the production of particles with desired sizes and shapes, we need to understand how the particle sizes and shapes are affected by these parameters quantitatively. We need to know the effect of the cutting-edge geometry on the penetration depth. In order to describe the process quantitatively, we also need to measure the material resistant force of UHMWPE in the sliding process of the cutting edges. Especially, the influence from the viscoelastic behavior of UHMWPE should be analyzed. More experiments need to be designed to provide a better understanding of the mechanism of particle generation quantitatively. 


\section{CHAPTER 8}

\section{MECHANISM OF UHMWPE PARTICLE GENERATION}

\subsection{Introduction}

The purpose of this chapter is to mechanistically elucidate the particlegeneration mechanism in the rubbing process between UHMWPE material and textured surfaces. To develop a predictive model for surface texture design, we need to understand the particle-generation mechanism. The first step is the quantification of the tip penetration into UHMWPE as a function of load. A nano-indenter was used to probe UHMWPE to observe the viscoelastic behavior of UHMWPE. After penetration, the sliding process was simulated with simple model experiments by observing in situ single asperity cutting process of UHMWPE via a microscopic video camera. From the results, we deduce the relationship between cutting depth, cuttingedge angle and particle generation processes.

We hypothesize that the particle-generation mechanism stems from: (a) Penetration of the tip; (b) Lateral displacement of the cutting edge; (c) Strain hardening of the polymer; (d) Embrittlement of the surface layer by molecular orientation, and (e) Detachment of the particle at the tip edge-material interface. We will show evidence in this chapter that these processes may actually take place. 


\subsection{Penetration of the cutting edge}

A fixed normal load was applied in the linear reciprocating wear process for UHMWPE particle generation. The cutting edges penetrated into UHMWPE at the beginning of the process. The penetration depth is determined by the normal load applied, angle and height of the cutting edges. When the normal pressure and material reaction force are balanced, a maximum penetration depth for that load is reached.

To examine the penetration process, a Nano Indenter XP (MTS Corporation) was used to examine the UHMWPE behavior ${ }^{1}$. The nano-indenter uses a compliance indentation system, capable of operating at loads in the microgram range, and the depth resolution is in the subnanometer range. Real-time indentation depth and load data were recorded during the process. A Berkovich tip (three-sided diamond pyramid) was used. The sides of the pyramid have an angle of $65.3^{\circ}$ normal to the base. Thus the indent marks appear as equilateral triangles, and the length of a side of a perfectly plastic indent is approximately 7.3 times its depth [98]. The indentation was carried out at a constant loading-unloading strain rate of $0.1 \mathrm{~s}^{-1}$. A holding time of $10 \mathrm{~s}$ was used between the loading and unloading stage. Figure 8.1 shows the loaddisplacement curve of the indentation.

${ }^{1}$ Technical assistance from Dr. Douglas Smith at NIST on nano-indentation tests is deeply appreciated. 


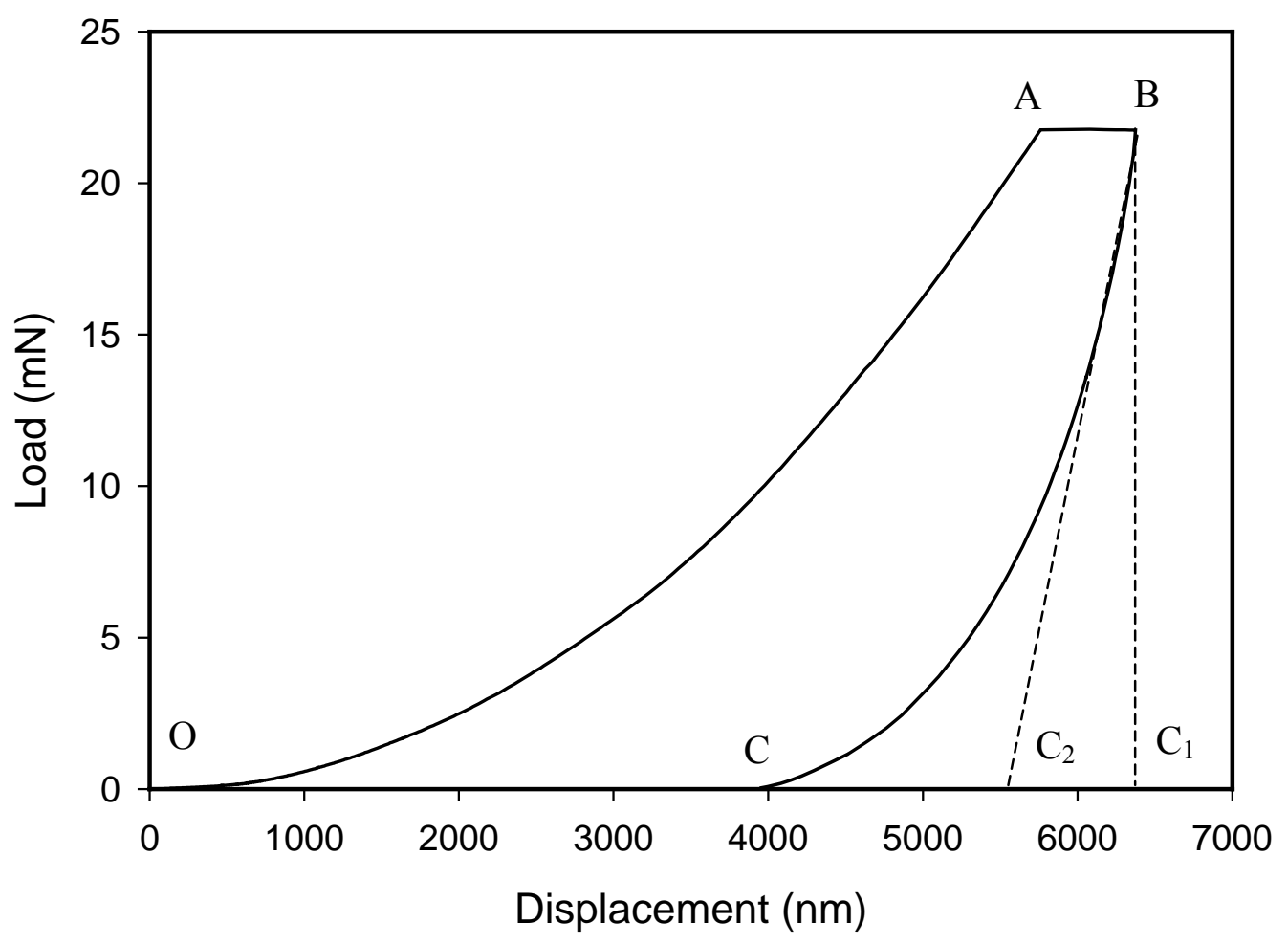

(Uncertainty of the measurement comes from surface roughness of the UHMWPE samples which is estimated to be less than $10 \%$ pf the measured value)

Figure 8.1 Load-displacement curve of nano-indentation test on UHMWPE with constant strain rate of $0.1 \mathrm{~s}^{-1}$. 
Figure 8.1 shows the displacement increases in the loading segment $(\mathrm{O} \rightarrow \mathrm{A})$. At the holding period with the constant load $(\mathrm{A} \rightarrow \mathrm{B})$, creep of the material under a constant stress was observed. During the unloading stage $(B \rightarrow C)$, the displacement curve shows additional deviation due to viscoelastic recovery of the material.

The data obtained from the unloading curve provide information regarding the elastic, viscoelastic and plastic behavior [98]. In Figure 8.1, the displacement at point $\mathrm{C}_{1}$ represents the maximum displacement $(6375 \mu \mathrm{m})$ of the indenter corresponding to the maximum load applied during the indentation, and the displacement at point $\mathrm{C}$ is the residual displacement after removing the indenter. The displacement at point $\mathrm{C}_{2}$ is the intercept of the tangent line drawn from the first part of the unloading curve, which describes the immediate recovery of the deformation once the stress is removed. The difference of the displacement between point $\mathrm{C}_{1}$ and $\mathrm{C}_{2}(812 \mu \mathrm{m})$ represents the elastic recovery of the material deformation. The difference of the displacement between point $\mathrm{C}$ and $\mathrm{C}_{2}(1563 \mu \mathrm{m})$ further indicates the delayed recovery of the material deformation by viscoelastic effect. Compared with the maximum penetration depth, $37.3 \%$ of the maximum penetration depth was recovered after the load is removed. Also comparing the ratio of the area " $\mathrm{BCC}_{1}$ " (viscoelastic recovery) to the area "OABC $C_{1}$ " (equal to the total energy done during the indentation) in Figure 8.1 shows that the effect of viscoelastic on UHWMPE deformation is dramatic. The viscoelastic recovery of material deformation of polymers under scratching was also revealed and measured by other investigators [99-103]. 
Elastic modulus of UHMWPE from the loading stage of the indentation test was calculated based on the stress and strain at certain penetration depth. Figure 8.2 shows the elastic modulus obtained from different penetration displacements. It shows that the elastic modulus near the surface is as high as $7 \mathrm{GPa}$. However, the deviation of the value near the surface may be up to $3 \mathrm{GPa}$ because of the roughness of the surface. The result still indicates that the elastic modulus evaluated at near-to-surface layers is much higher than the bulk material. A higher elastic modulus of UHMWPE surface layer was also indicated by Briscoe et al. [98]. The UHMWPE samples were prepared by a machining process. This may be due to the higher hardness of the surface layer from machining process. The shear stress applied on the surface may induce the molecular orientation and make the material surface harder. We will further discuss the molecular orientation of UHMWPE in the later section. The perturbation of the results can also be due to the roughness of the UHMWPE surface.

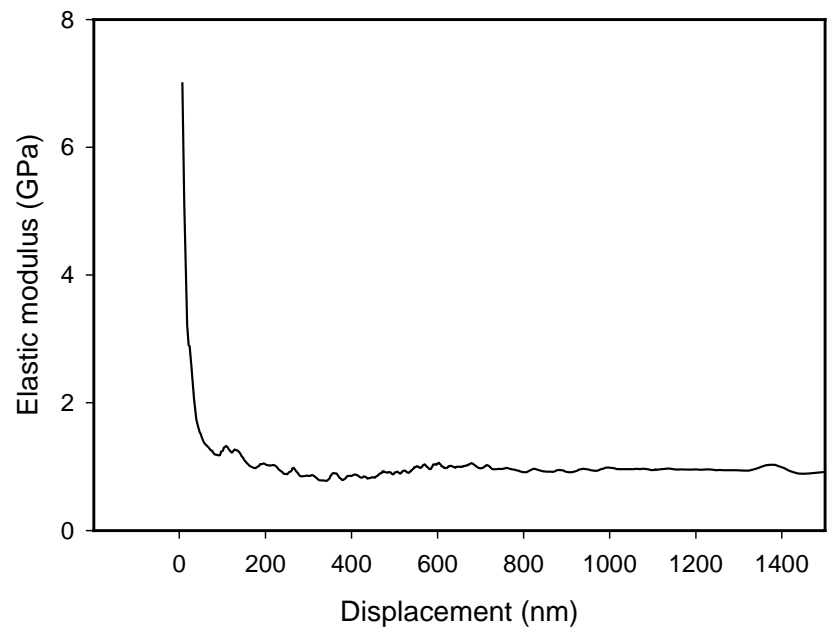

(Uncertainty of the measurement comes from surface roughness of the UHMWPE samples which is estimated to be less than a relative standard uncertainty of $10 \%$ )

Figure 8.2 Elastic modulus data for UHMWPE under different penetration depths from a nano-indentation test (strain rate $=0.1 \mathrm{~s}^{-1}$ ). 
It is seen from Figure 8.2 that the elastic modulus reaches a steady value of around $1 \mathrm{GPa}$ when the penetration depth is larger than $500 \mathrm{~nm}$. This value will be used later.

We also measured the penetration depths of the wedge-shaped tip with different angles under various normal loads. The wedge-shaped tip will be later used in the simple model experiments of the sliding process. Two wedge tips are $90^{\circ}$ and $60^{\circ}$ with $2 \mathrm{~mm}$ edge length. The normal load was applied and held for $15 \mathrm{~s}$. Given sufficient time $(60 \mathrm{~min})$ after the indentation tests, profiles of the indentation marks were measured by profilometry. The final penetration depths of the various normal loads and cutting-edge angles are listed in Table 8.1. Obviously, the penetration depth increases with increasing applied normal load. A smaller cutting-edge angle (sharper edge) leads to a deeper penetration under the same normal load. This result may be due to the larger local compression stress induced by a sharper edge.

Table 8.1 Comparison of penetration depth for the cutting edges with different angles under various normal loads.

\begin{tabular}{|l|c|c|c|}
\hline \multirow{2}{*}{} & \multicolumn{3}{|c|}{ Final penetration depth } \\
\cline { 2 - 4 } & $\begin{array}{c}\text { Normal load } \\
=22.25 \mathrm{~N}\end{array}$ & $\begin{array}{c}\text { Normal load } \\
=44.50 \mathrm{~N}\end{array}$ & $\begin{array}{c}\text { Normal load } \\
=66.75 \mathrm{~N}\end{array}$ \\
\hline angle $=60^{\circ}$ & $(29.6 \pm 2.1) \mu \mathrm{m}$ & $(56.8 \pm 3.5) \mu \mathrm{m}$ & $(85.2 \pm 4.9) \mu \mathrm{m}$ \\
\hline angle $=90^{\circ}$ & $(21.9 \pm 2.0) \mu \mathrm{m}$ & $(47.7 \pm 2.9) \mu \mathrm{m}$ & $(70.6 \pm 4.5) \mu \mathrm{m}$ \\
\hline
\end{tabular}




\subsection{Sliding of the cutting edge}

To understand the mechanism of particle generation, a direct observation of the cutting-edge sliding process would be desirable. However, the small scale of surface textures makes in situ observation difficult. A scaled-up model experiment was developed to allow the direct observation of the particle-generation process under a constant load.

\subsubsection{Simple model experiments with constant load}

The experimental setup of the scaled-up single cutting-edge sliding test is schematically shown in Figure 8.3. A wedge-shaped tip was fabricated out of tool steel. Two different tips ( $90^{\circ}$ and $60^{\circ}$ with $2 \mathrm{~mm}$ edge length) were prepared. The tip was mounted on a lever arm. A UHMWPE disk (5.08 $\mathrm{cm}$ (2") in diameter) was mounted in the lower holder attached to a horizontal slider. The slider was driven by a rotary motor at an average sliding speed of $(57.2 \pm 3.2) \mathrm{mm} / \mathrm{s}$ (the same speed in the particle- generation process).

A piezoelectrical three-dimensional force transducer was mounted above the tip to measure forces. The load cell sent electrical signals from the applied forces. The electrical signals were sent to three charge amplifiers. The output of the amplifier could be adjusted from $1 \mathrm{~N} / \mathrm{V}$ to $1000 \mathrm{~N} / \mathrm{V}$ and the voltage signal was then stored by the data-acquisition system. Calibration was made via prior the tests to obtain the curve of output voltage versus load. 
To observe the sliding process, a video camera (Digital 5000 by Panasonic) was mounted on the frame of the test rig. A pair of micro lenses, made by Volpi Company was used. The magnifications are 165 times and 300 times for the two lenses and the focus distance between the lens and the specimen is $50 \mathrm{~mm}$. The video pictures picked up by the camera were recorded by a DV system (Sony). Later on, the recorded video could be converted as a digital file to the computer. Thus we can show the video frame by frame allowing the dynamic sliding process to be analyzed. A fiberglass light source was applied. The light source was arranged behind the specimen to give a bright and clear image of the material in front of the cutting edge.

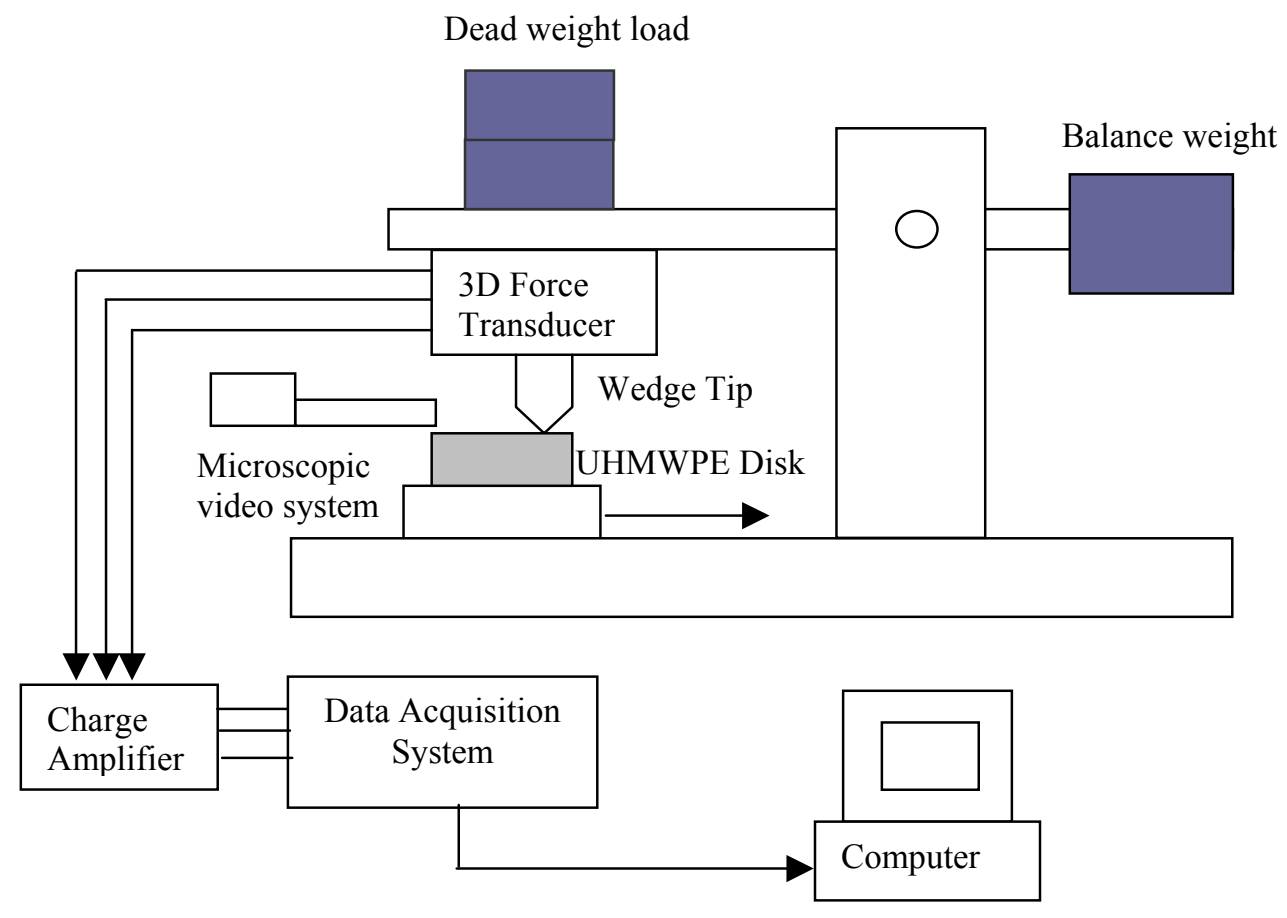

Figure 8.3 Schematic representation of scaled-up single cuttingedge sliding test with fixed normal-load condition. 


\subsubsection{Lateral displacement of the tip}

Various normal loads $(22.25 \mathrm{~N}, 44.50 \mathrm{~N}$, and $66.75 \mathrm{~N})$ were applied on the wedge-shaped cutting edges with two different included angles $\left(60^{\circ}\right.$ and $\left.90^{\circ}\right)$ under a average sliding speed of $57.2 \mathrm{~mm} / \mathrm{s}$. The video observation shows that the cutting edge moves up and down during the sliding process. Examples of the video frames are shown in Figure 8.4. It can be seen that the cutting edge penetrates into the material in Figure 8.4(a). It is observed that the cutting edge is then pushed up away from the surface in the following frames (Figure 8.4(b)). Then the cutting edge penetrates into the material again. The phenomena were repeated during the entire sliding process.

We hypothesize that the lift-up force comes from the material strain-hardening of the UHMWPE and the accumulated polymer in front of the cutting edge. After the cutting edge is lifted up, there is no contact between the cutting edge and the material surface, the material resistant force (The force acting on the cutting-edge during the sliding process) drops to zero. The normal load then pushes the cutting edge back into the UHMWPE material again. This process is repeated during the process. No wear particle was observed for the first cycle. After several cycles, generation of wear particels were observed. Based on this observation, the cutting-edge sliding process can be summarized and is shown in Figure 8.5. 


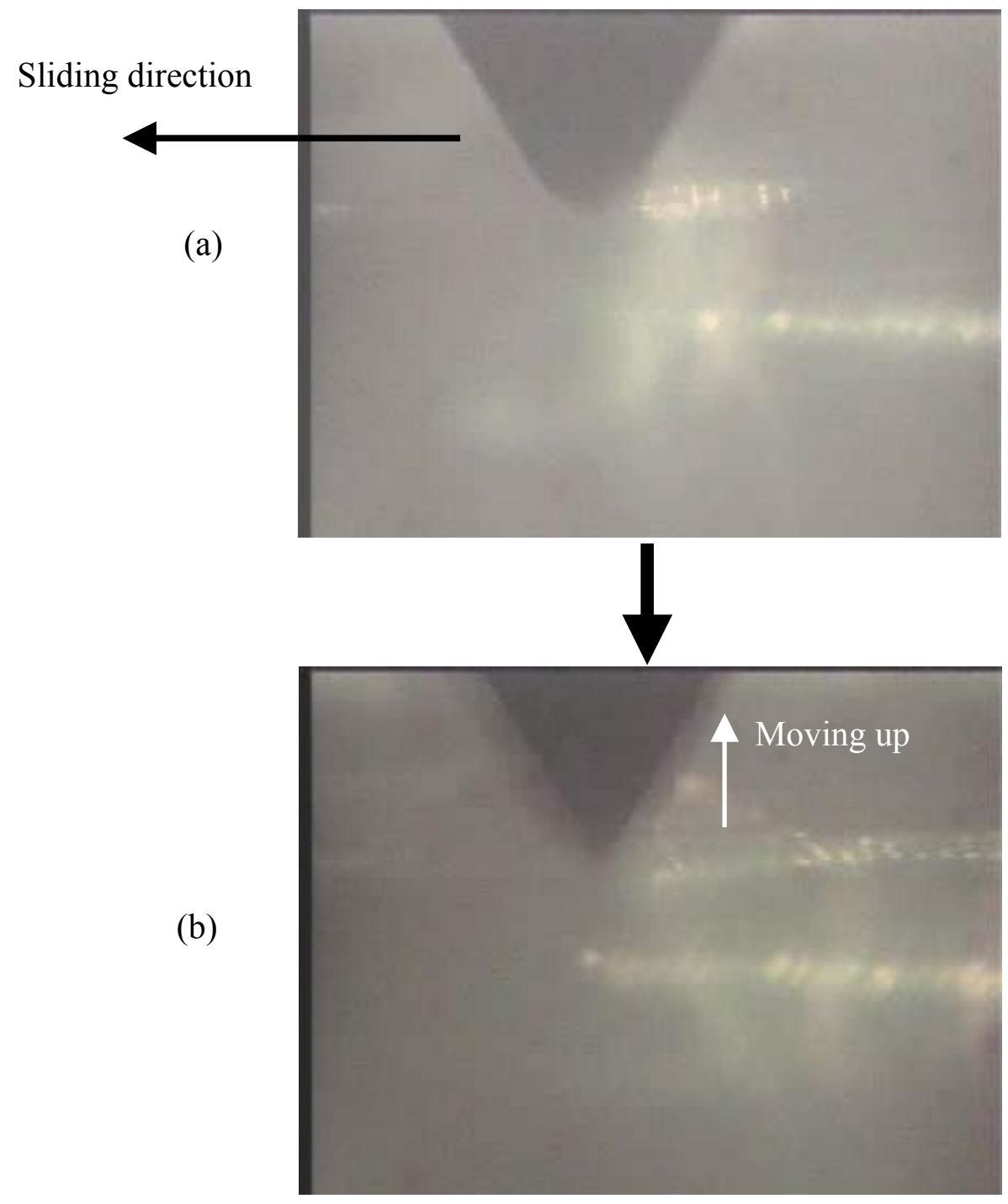

Figure 8.4 Video frames of a cutting edge lift-up process. 
(a)

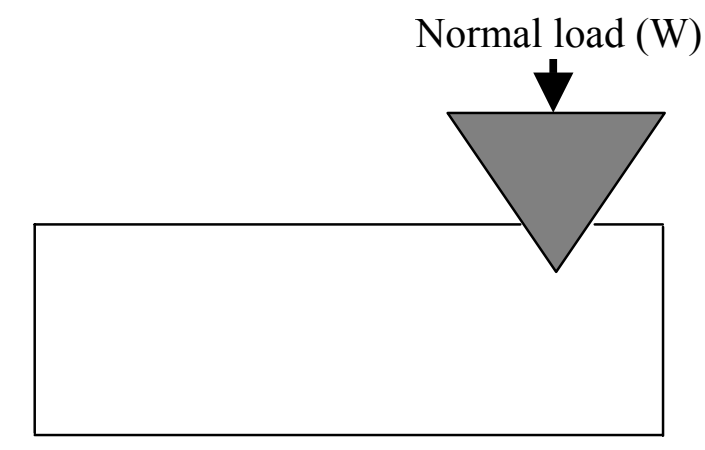

(b)

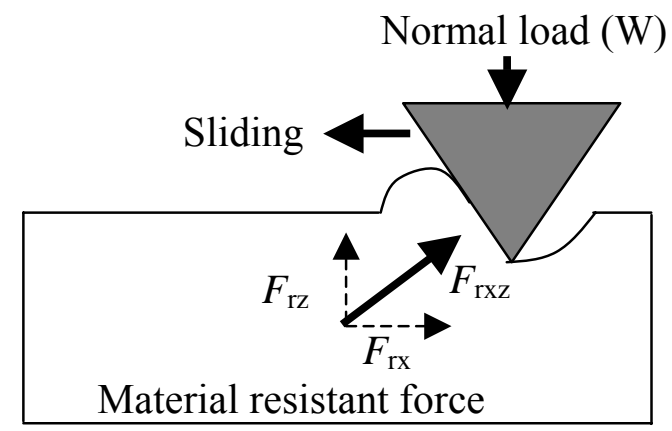

(c)

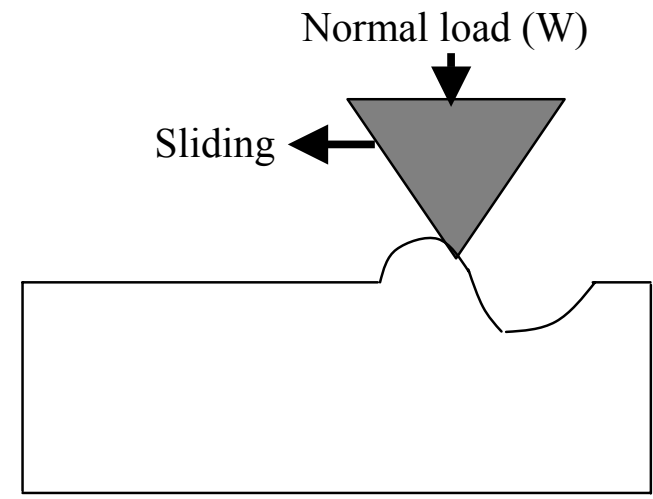

(d)

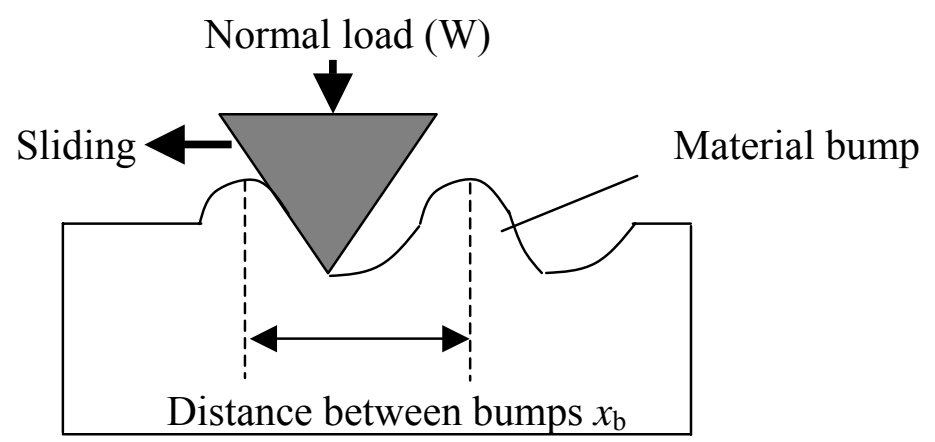

Figure 8.5 Schematic representation of the cutting-edge sliding process over UHMWPE material with fixed normal load. 


\subsubsection{Reacting forces}

Normal force and horizontal force were recorded during the cutting edge sliding process $\left(F_{\mathrm{x}}, F_{\mathrm{z}}\right)$. Figure 8.6 shows the force curve of the sliding process by a wedge-shaped cutting edge with an included angle of $90^{\circ}$ and Figure 8.7 is the force curve of the sliding process by a wedge-shaped cutting edge with an angle of $60^{\circ}$.

When the sliding process begins, the material resistant force acting on the cutting edge increases with increasing deformed UHWMPE accumulated in front of the cutting edge. Thus the horizontal force acting on the cutting edge increases.

The initial vertical force shown in the plot represents the applied normal load of the test. We define $F_{\mathrm{rxz}}$ is the resultant resistant force acting on the cutting edge. $F_{\mathrm{rz}}$ and $F_{\mathrm{rx}}$ are the resolved component forces in the vertical and horizontal directions, respectively (see Figure 8.5). $F_{\mathrm{rz}}$ contributes to the lift-up force of the cutting edge. First $F_{\mathrm{rz}}$ increases with increased accumulated volume of UHMWPE, and $F_{\mathrm{rz}}$ also contributes to lift up the cutting edge in the sliding process. The contact area between the cutting edge and the material decreases during the lift-up process and thus the $F_{\mathrm{rz}}$ starts to drop. At the moment that the cutting edge is pushed to the apex of the material bump, $F_{\mathrm{rz}}$ drops to zero. According to the fact that the vertical force shown on the plot presents the difference between the normal force and the material resistant force in the vertical direction. Thus the amount of vertical force goes back to the original applied normal load when the cutting edge is fully retracted. The reactingforce curves do conform to the hypothesized mechanism as we proposed after viewing the video recording of deformation in Figure 8.5. 


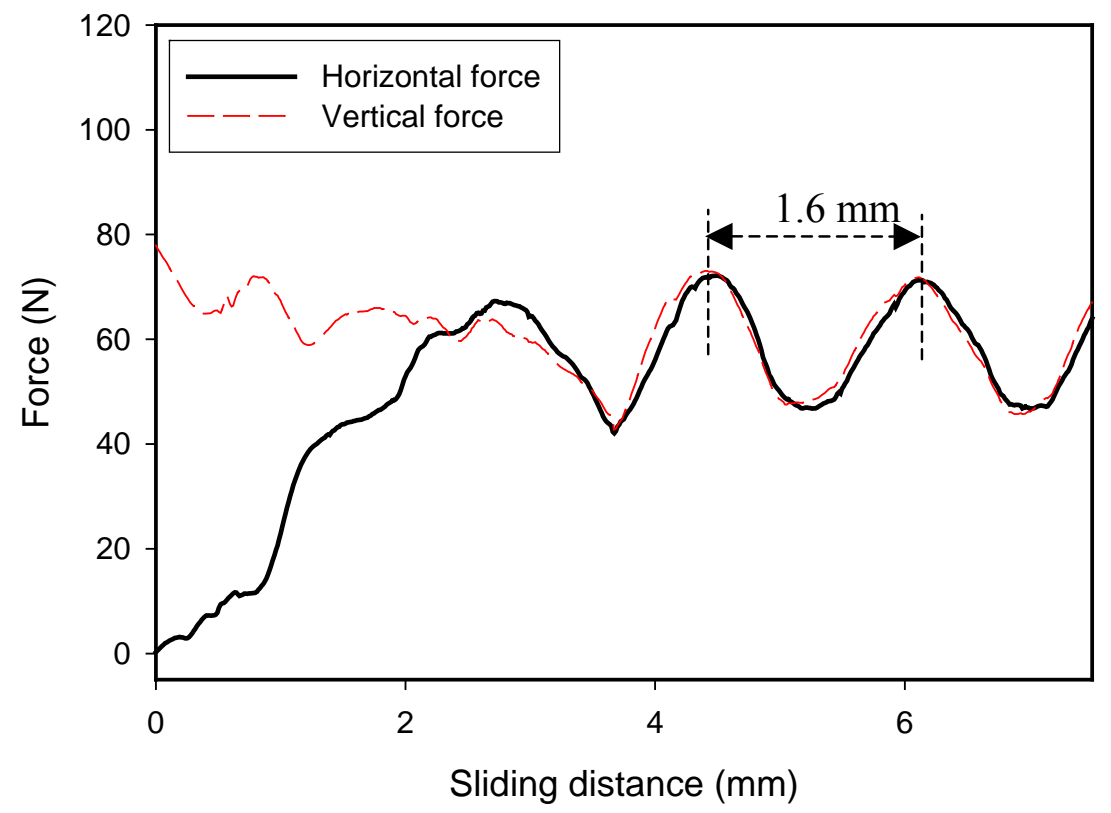

Figure 8.6 Force curve of the sliding process by a $90^{\circ}$ wedge-shaped cutting edge under a normal load of $66.75 \mathrm{~N}$.

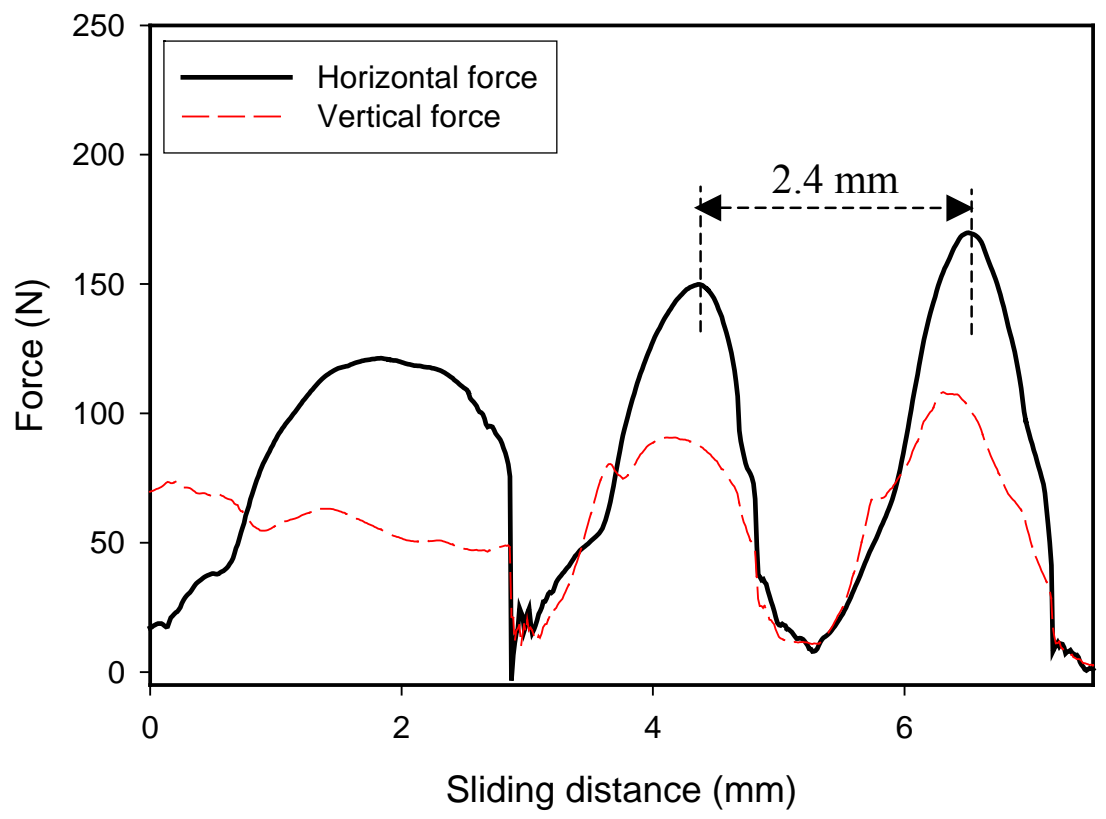

Figure 8.7 Force curve of the sliding process by a $60^{\circ}$ wedge-shaped cutting edge under a normal load of $66.75 \mathrm{~N}$. 


\subsubsection{Effect of normal load and cutting-edge angle}

The frequency of the up and down movement of the cutting edge or the distance between the repeated material bumps provides information on the shear deformation of the material during the sliding process. Thus by understanding the force curve of the sliding process, the influence of the geometry of the cutting edge and mechanical operation conditions on the wear-particle dimensions can be understood.

The sliding distances between two displaced bumps under different normal loads and cutting-edge angles are plotted in Figure 8.8. It shows that increasing normal load results in a longer sliding distance. Under the same normal load, a sharper cutting edge (smaller cutting edge angle) results in a longer sliding distance. A linear relationship between the sliding distance and normal load is observed.

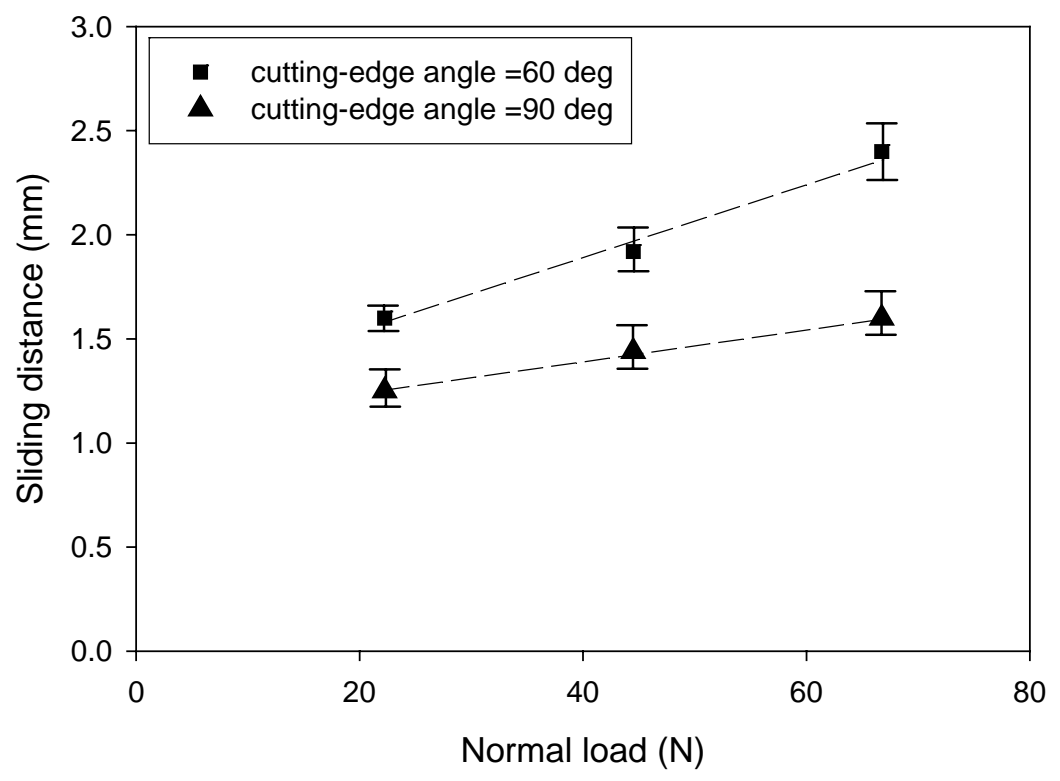

Figure 8.8 Sliding distance between two peaks under different normal loads with two cutting-edge angles. 
Dependence of the cutting-edge lift-up process on the cutting-edge angle needs further examination. As illustrated in Figure 8.9, the quantity of the resolved component forces in vertical direction is a function of the cutting-edge angle. Assuming the same penetration depth during the sliding process, the resultant material resistant forces $\left(F_{\mathrm{rxz}}\right)$ from the shear of UHMWPE material are the same. The resolved force in the vertical direction is $F_{\mathrm{rz}}=F_{\mathrm{rxz}} \sin \theta$ as shown in Figure 8.9. A larger angle causes more material resistant force distribute to the vertical direction that increases the lift-up force. Thus the time required for the cutting edge to be pushed away is shorter. With a constant sliding speed in the horizontal direction, a shorter distance between two peaks is expected. Variation of the cutting-edge angle contributes to the change of penetration depth and the cutting edge lift-up speed. Both consequences have an influence on the sliding distance.

To sum up, the effects of normal load and cutting-edge angle on the sliding process and, hence, on the particles dimensions are elucidated from the results of simple model experiments. However, the generation of particles was not observed from a single pass of sliding process. Particles were generated only after several cycles of sliding processes. We suspect this may be due to embrittlement of UHMWPE from strain hardening and molecular orientation during the repeated sliding processes. 


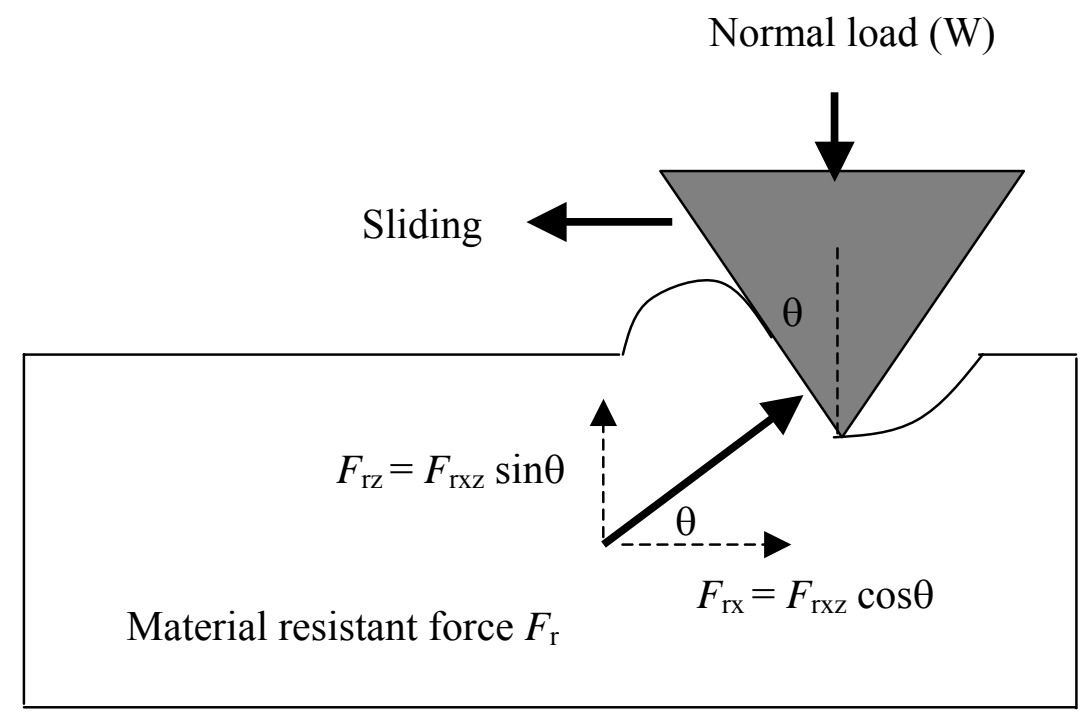

Figure 8.9 Schematic representation of the resolved component forces of the material resistant force in the vertical and horizontal direction during the sliding process. 


\subsection{Shear induced molecular orientation of UHMWPE}

UHMWPE is a semi-crystalline material and the folded-chains in the crystalline region have a specific molecular orientation. The chains tend to align and orient to a common direction when the surface is subjected to a directional force such as shear stress from sliding.

X-ray absorption spectroscopy (XAS), in the form of near-edge x-rayabsorption fine-structure (NEXAFS) or x-ray-absorption near-edge structure (XANES), has demonstrated its ability to measure the orientation of hydrocarbon molecules at the surface [26]. This technique involves the resonant x-ray excitation of an electron to an unoccupied low-lying molecular orbital. The initial-state K-shell excitation gives XAS its element specificity, and the final-state unoccupied molecular orbitals give XAS its bonding selectivity. A measurement of the intensity of XAS spectral can identify chemical bonds and determine their relative population density within a sample. The amount of soft $\mathrm{x}$-ray absorption is very sensitive to the average molecular chain angle with respect to the electric field vector (E) of the incident x-ray beam. This variation allows a measurement of the average molecular orientation of the alkyl chain with respect to the surface of UHMWPE.

Soft x-ray absorption-spectroscopy was used [27-28] to measure the molecular orientation at UHMWPE surfaces subjected to different shear motions by rubbing a UHMWPE pin against a polished Co-Cr disk ${ }^{2}$.

\footnotetext{
${ }^{2}$ Experiments on molecular orientation of UHMWPE were conducted by Dr. Daniel Fischer, and Dr. Ming Shen from NIST and Dr. Sharadha Sambasivan from Brook Haven National Laboratory.
} 
Figure 8.10 is the partial electron yield of a sample subjected to soft $\mathrm{x}$-ray beams after 5000 unidirectional sliding cycles. The differences between the spectra quantify the degree of polymer chain alignment on UHMWPE surface. Figure 8.11 further illustrates the difference spectra of UHMWPE after 5000 unidirectional sliding cycles. A larger difference between the spectra reflects a higher degree of molecular orientation. The results show that a higher degree of chain alignment was induced by a uni-directional rubbing the UHMWPE surface.

Linear reciprocating wear testing was conducted in our UHMWPE particlegeneration processes. We think that the UHMWPE molecules align to the direction of shear. The molecular orientation causes the UHMWPE to behave more brittle. This agrees with the nano-indentation result as shown in Figure 8.2.

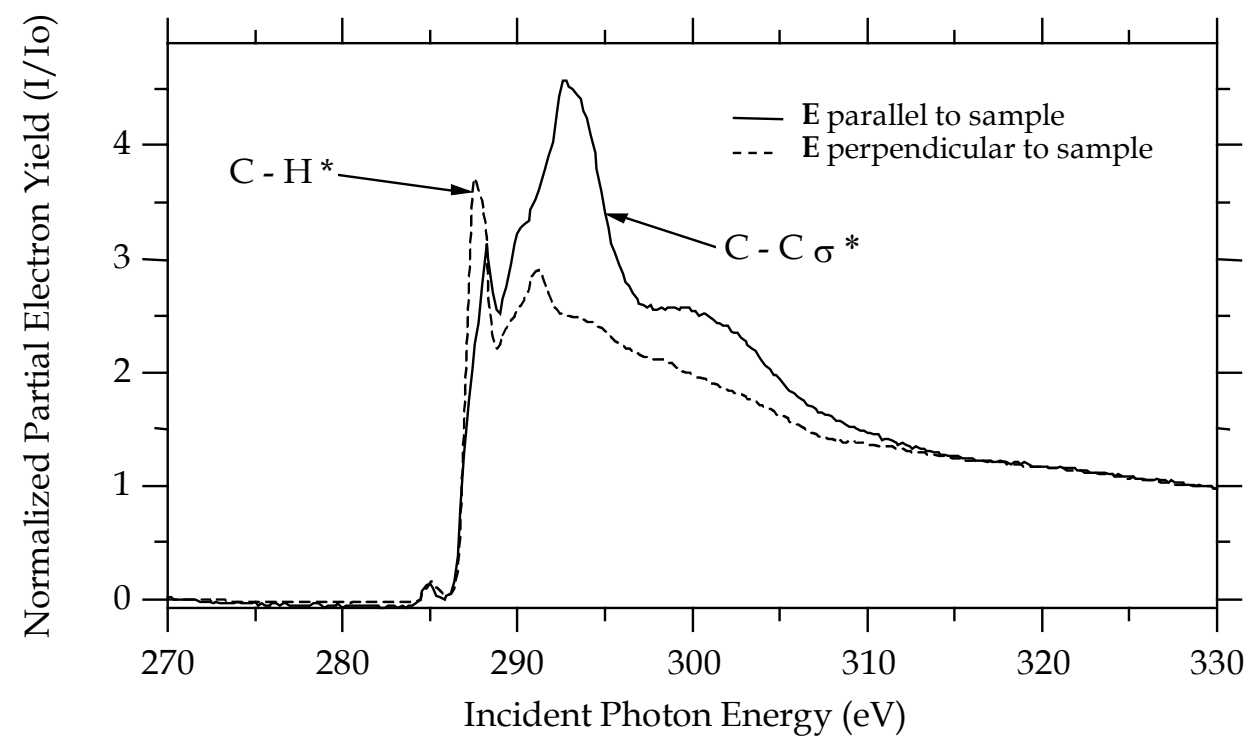

(The uncertainty of the measurement is estimated to be within 5\%)

Figure 8.10 Soft $x$-ray absorption spectra showing the molecular orientation of surface layer after 5000 unidirectional sliding cycles. $\mathbf{E}=$ electric field [27]. 

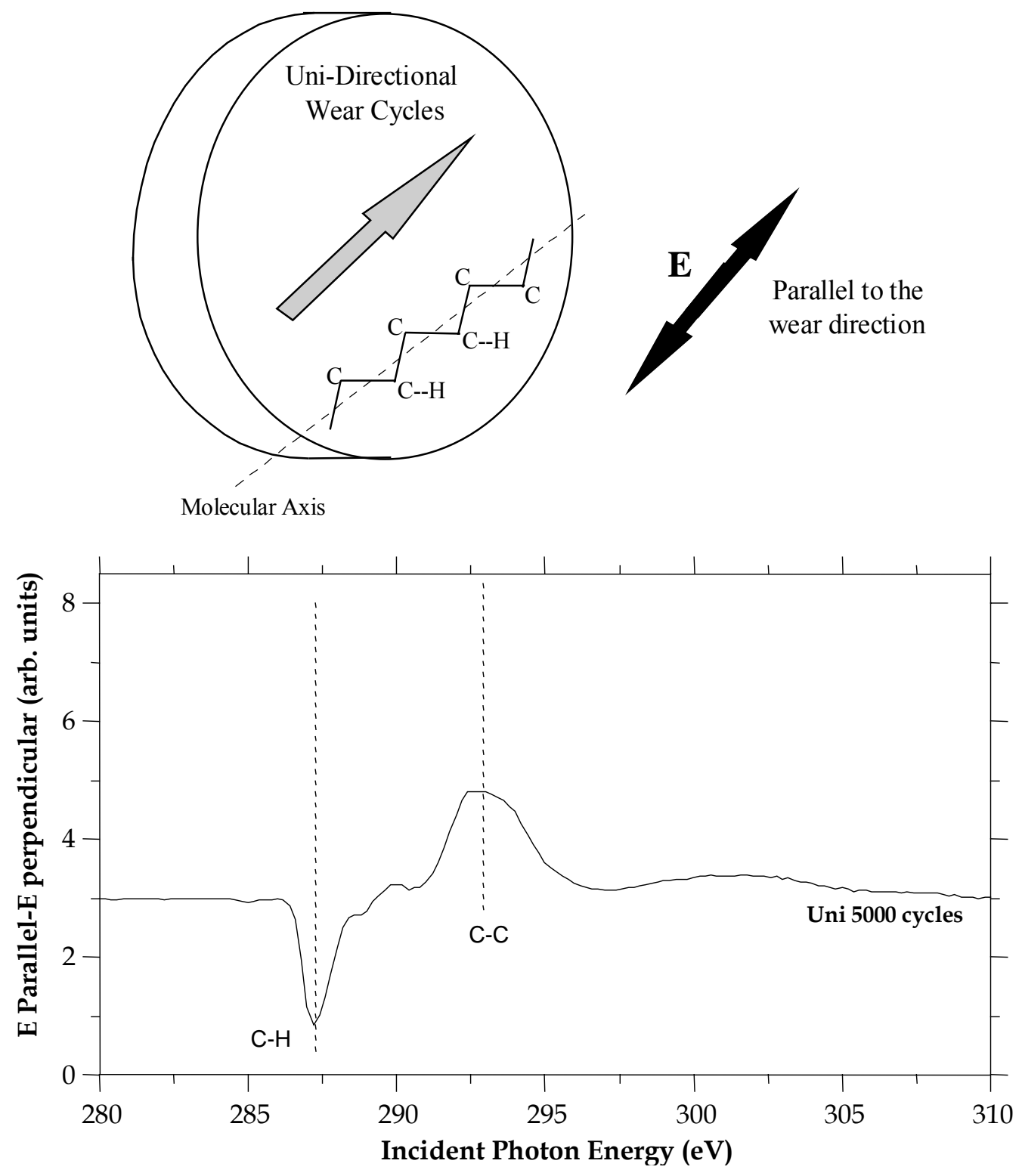

(The uncertainty of the measurement is estimated to be within $5 \%$ )

Figure 8.11 Soft $x$-ray absorption spectra showing the degree of molecular alignment of UHMWPE surface layer after 5000 unidirectional sliding cycles [26]. 


\subsection{Strain-hardening effect}

A strain-hardening effect of UHMWPE in the plastic deformation regime has been identified and measured in several studies [104-107]. Kurtz et al. [104] have studied the yielding and plastic-flow behavior medical grade of UHMWPE (GUR 4150) by conducting uniaxial tension and compression tests. GUR 4150 UHMWPE was also used in our studies. They showed that the equivalent true stress-strain behaviors from tension and compression tests were similar. A true stress-strain relationship was obtained in Figure 8.12 with a strain up to 1.5 . Dramatic strainhardening was observed. Figure 8.13 shows the true stress-strain relationship of GUR 4150 UHMWPE up to a 0.12 true strain. The yield point was identified and a strainhardening effect was observed with increasing stress needed for the increasing plastic strain. An elastic-plastic model was then proposed especially to describe the strain hardening behavior of the plastic deformation of UHMWPE [104].

We propose that the material is accumulated in front of the cutting edge during the sliding process. The material resistant force arises because of increasing volume of UHMWPE accumulated in front of the cutting-edge surface. The material is then strain hardened and increases resistant force acting on the cutting-edge surface. Depending on the angle of the surface, the force is then resolved into a vertical force and a horizontal force. The vertical force then begins to push the cutting edge up. Thus we revised the simple model experiment to a constant depth sliding process to measure this resistant force. 


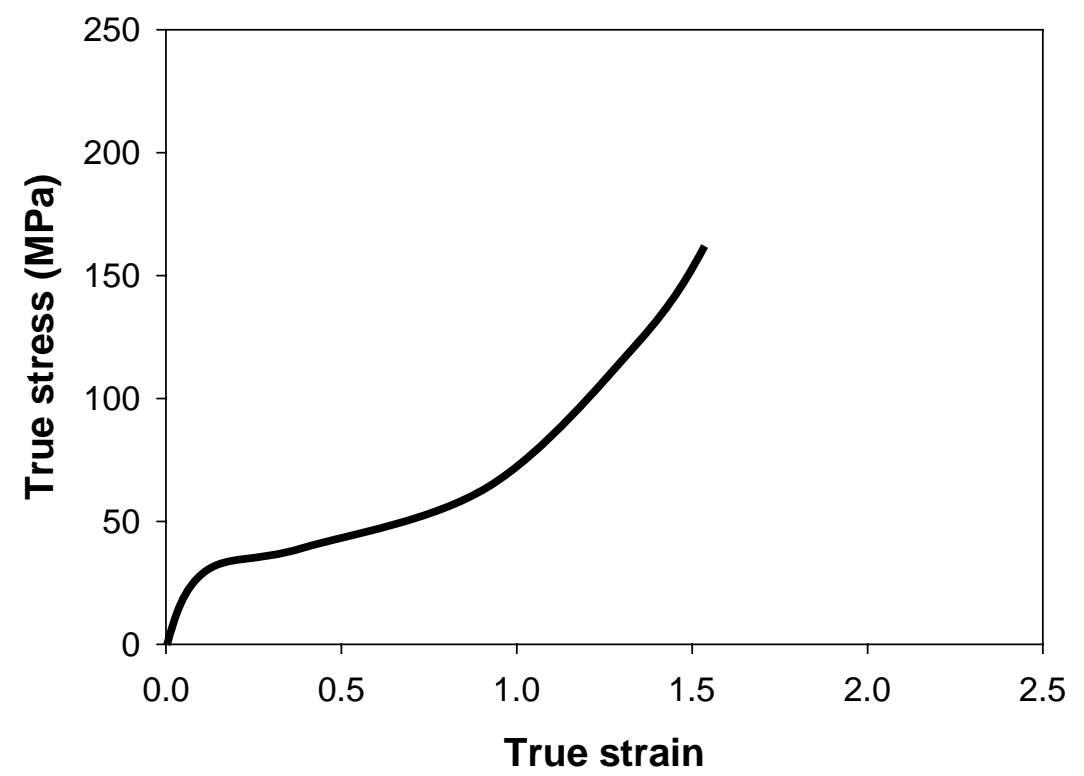

Figure 8.12 True stress-strain curves for UHMWPE (GUR 4150) [104].

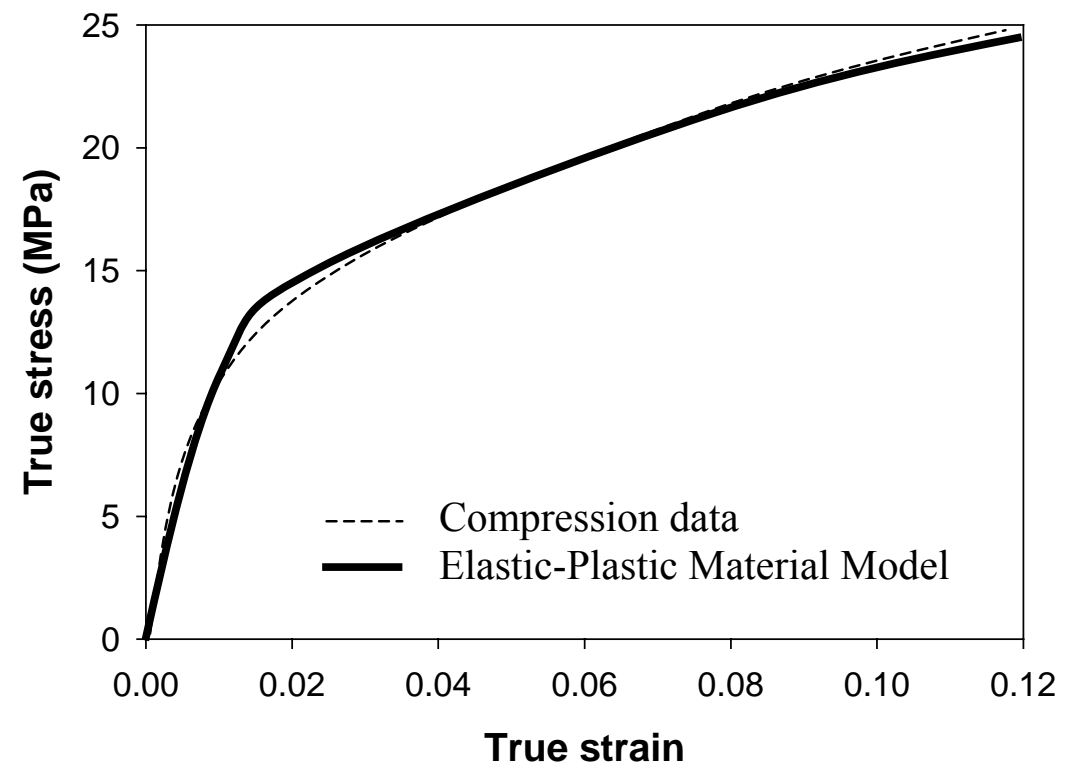

Figure 8.13 True stress-strain curves for UHMWPE 4150 up to 0.12 strain in uniaxial tension and compression and elastic-plastic model to describe the behavior [104]. 


\subsubsection{Constant depth sliding model experiments}

To determine the displaced volume of UHMWPE before the cutting edge is lifted up, the simple model experiment under constant load was modified to a process with constant depth of sliding. The purpose is to measure the maximum material resistant force by pre-defining the displaced volume and by monitoring the material deformation in-situ.

The experimental set-up of constant depth sliding tests is shown schematically in Figure 8.14. The cutting edge is held in a fixed position on a vertical slider that can be adjusted in the vertical direction to control the penetration depth. A UHMWPE disk (2" in diameter) is mounted in a lower holder on a horizontal slider in the Y direction. The Y direction slider is attached to another horizontal slider in the $\mathrm{X}$ direction. The sliders are driven by a micro-stepping motor. This is controlled by a computer. The vertical and horizontal sliders move at the precise distance, speed, and acceleration specified by the computer controller. One motion controller determines the motion of vertical slider ( $Z$ direction), and the other motion controller operates the motion of two horizontal sliders in the $\mathrm{X}$ and $\mathrm{Y}$ directions. We used the same force recording and microscopic video system as described in section 8.3.1. 


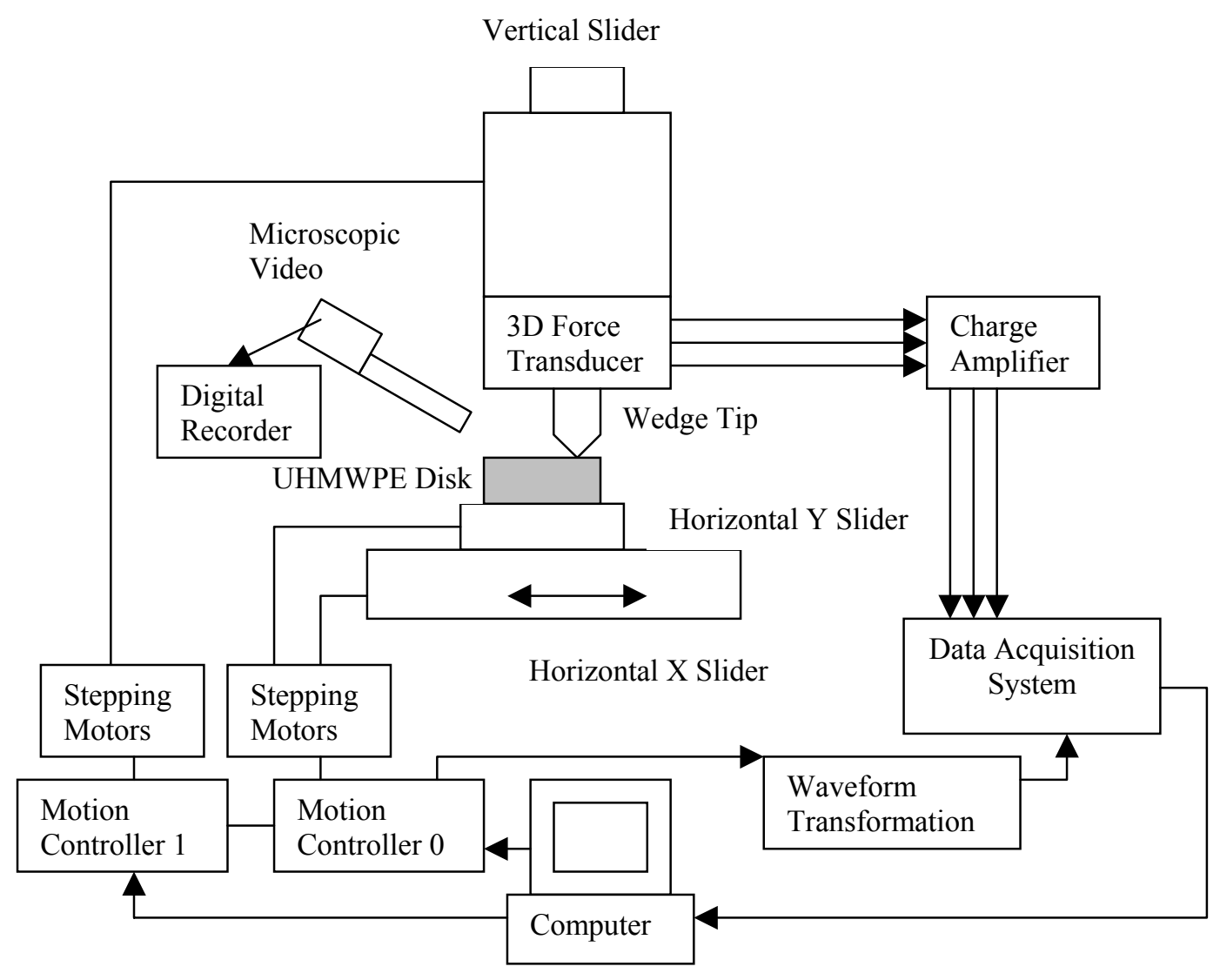

Figure 8.14 A schematic representation of the displacement controlled single cutting edge sliding test. 
Wedge-shaped cutting edges with two cutting-edge angles $\left(60^{\circ}\right.$ and $\left.90^{\circ}\right)$ were used. The cutting edge length is $2 \mathrm{~mm}$. The contact between the cutting edge and the UHMWPE surface can be detected from the on-line monitoring of the forces. The UHMWPE sample was leveled first on the stage before the tests. The indentation of the cutting edge was conducted at a speed of $0.5 \mathrm{~mm} / \mathrm{s}$ vertically. Various penetration depth (ranging from $80 \mu \mathrm{m}$ to $400 \mu \mathrm{m}$ ) were applied in the tests. After $2 \mathrm{~s}$, the cutting edge was slid sideways for $4 \mathrm{~mm}$ with a constant speed of $0.5 \mathrm{~mm} / \mathrm{s}$.

Figure 8.15 shows a typical horizontal-force curve for a $60^{\circ}$ cutting edge with a penetration depth of $400 \mu \mathrm{m}$. The sliding motion starts at time $t=0$. The horizontal force acting on the cutting edge grows and approaches a steady value. The video of the cutting-edge sliding process was synchronized with the force curve in Figure 8.16. By comparing the volumes of accumulated material in the video frames, it is observed that a maximum accumulated volume was achieved between $t=0.8 \mathrm{~s}$ and $t=1.2 \mathrm{~s}$ which corresponds to the point $\mathrm{A}$ in the force cure in Figure 8.15. After $t=1.2 \mathrm{~s}$, the accumulated material volume in front of the cutting edge remains the same. Plastic flow of the material into the near-by region continues during the following cuttingedge sliding process. By identifying the time for reaching the maximum accumulated volume, the sliding distance toward this point is determined with the known sliding speed. The maximum displaced volume of the material during the sliding process is equal to the product of cutting-edge length, fixed penetration depth and the sliding distance. 


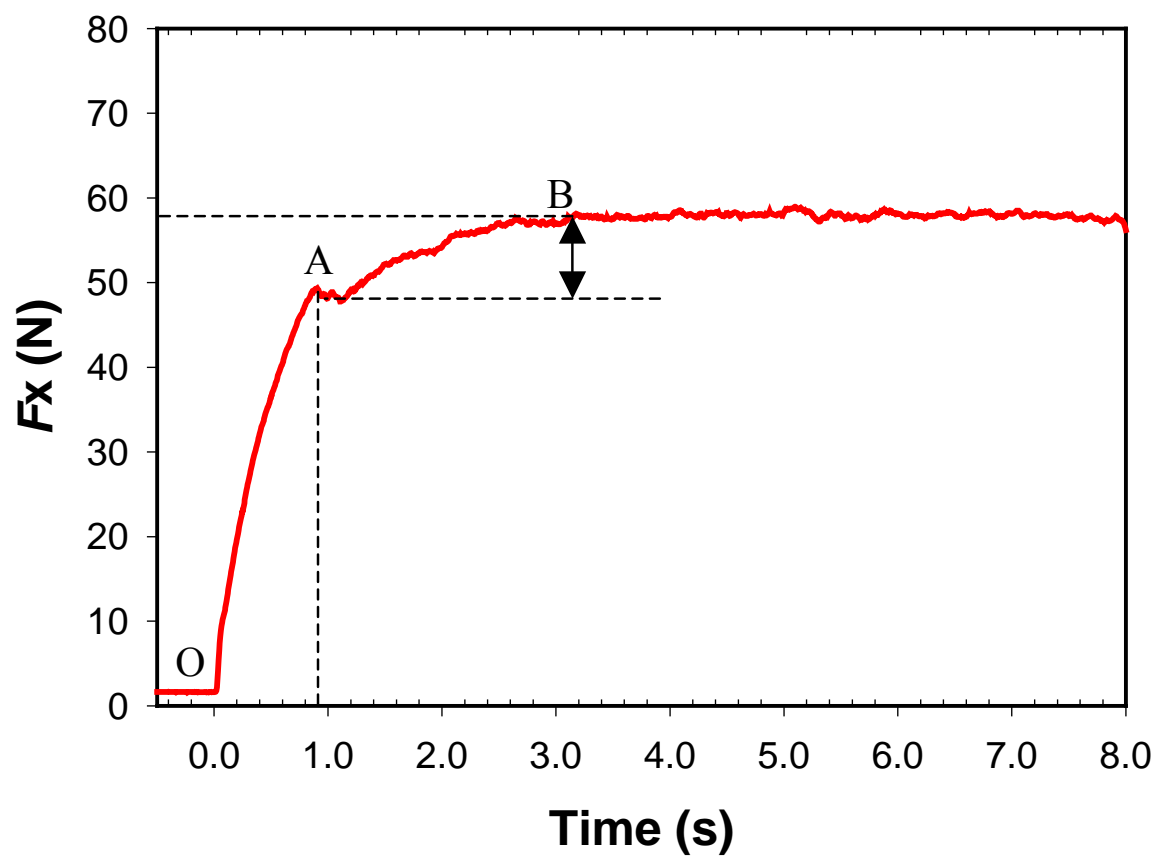

Figure 8.15 Horizontal-force curve of the cutting-edge sliding process (cutting-edge length $=2 \mathrm{~mm}$, penetration depth $=400 \mu \mathrm{m}$, sliding speed $=0.5 / \mathrm{s}$ ) 

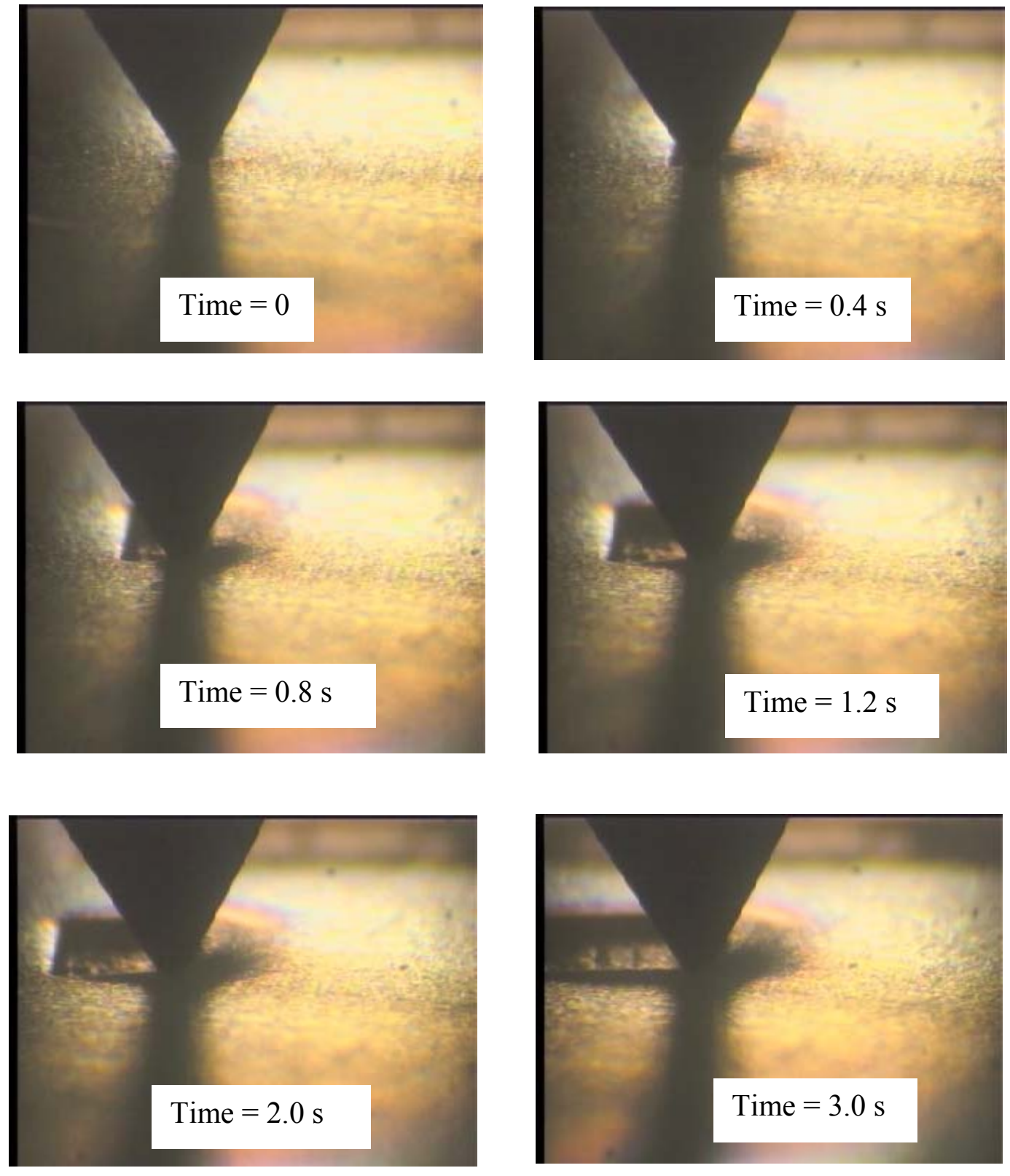

Figure 8.16 Video frames of the cutting edge sliding process (cutting edge length $=2 \mathrm{~mm}$, penetration depth $=400 \mu \mathrm{m}$, sliding speed $=0.5 / \mathrm{s})$. 
With these observations, we may explain the sliding process as follows. From point $\mathrm{O}$ to $\mathrm{A}$ in Figure 8.15, the sliding process produces shear of UHMWPE and the material pile-up is accumulated in front of the cutting edge. The increased deformation volume in front of the cutting edge leads to an increase of the material resistant force acting on the cutting edge. Thus an increased horizontal force is observed. The material accumulated in front of the cutting edge reaches a maximum volume at point A. This maximum displaced volume is projected to be the particle volume in the particle-generation process.

From point $\mathrm{A}$ to $\mathrm{B}$, the horizontal force keeps growing without a visible increase of the cumulative volume in front of the cutting edge, but an increasing sidewall of material is observed. The material displaced by the cutting edge no longer goes in front of the cutting edge. It is suggested that the cutting edge keeps pushing the UHMWPE material and the strain-hardening effect of plastic deformation contributes to the increase of the horizontal force from A to B. Since the depth of penetration is fixed in the experiment, the increased force is not able to lift up the cutting edge. Finally, the increased contact pressure of the cutting edge face forces a plastic flow of materials sideways. A steady state plastic flow is established and the horizontal force approaches a constant value as seen after point B in Figure 8.15. 
Experiments conducted at various penetration depths and with different cutting edge angles are discussed in this section. We compare the effect from different penetration depths on the sliding distance in Figure 8.17. The sliding distance toward the maximum displaced volume for a $160 \mu \mathrm{m}$ penetration depth is $340 \mu \mathrm{m}$ and for a $80 \mu \mathrm{m}$ penetration depth it is $162 \mu \mathrm{m}$. The measured values have a relative standard uncertainty of $\pm 5 \%$. The larger penetration depth also leads to a larger sliding distance toward the maximum displaced volume and also leads to a larger maximum displaced volume during the process.

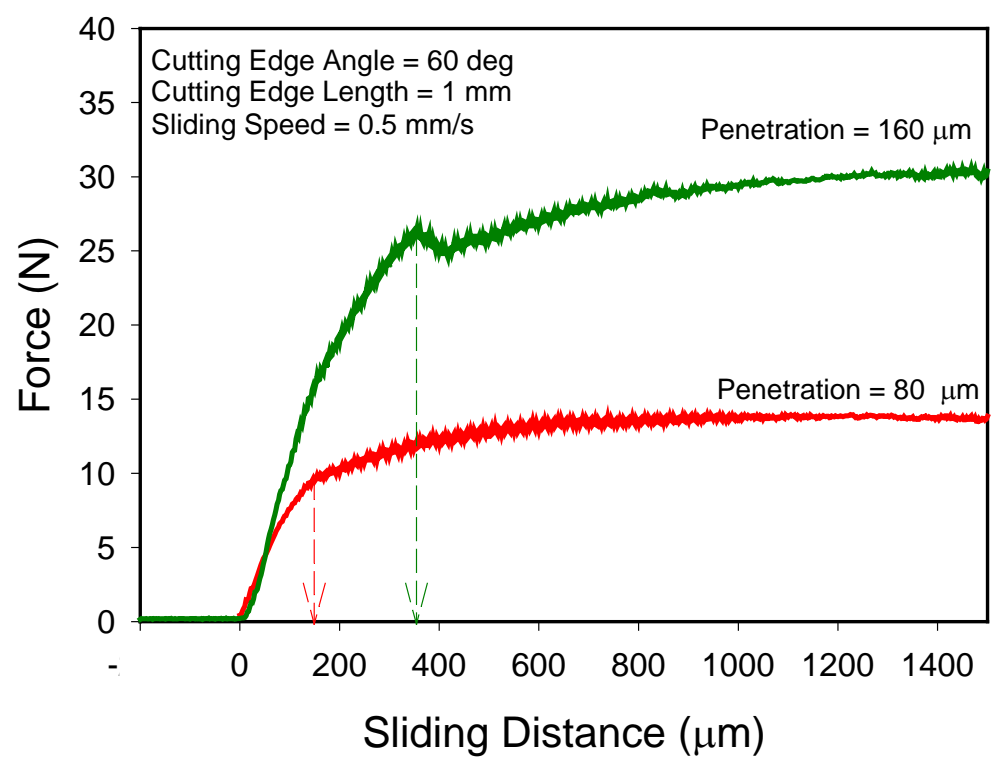

Figure 8.17 Horizontal force versus sliding distance for different penetration depths in a displacement-controlled cutting-edge sliding test. 
We compare the experiments with different cutting-edge angles in Figure 8.18. It is seen that a sharper cutting edge $\left(60^{\circ}\right)$ leads to a larger sliding distance toward the maximum displaced volume. The maximum resistant force for the sharper cutting edge $\left(60^{\circ}\right)$ is also larger. We suggest that the resistant force contributing to the sliding direction is larger for the cutting edge with a smaller angle. More volume space in front of the cutting edge (especially in the vertical direction) is reserved for the material deformation and accumulation. Thus a long sliding distance toward the maximum displaced volume is expected.

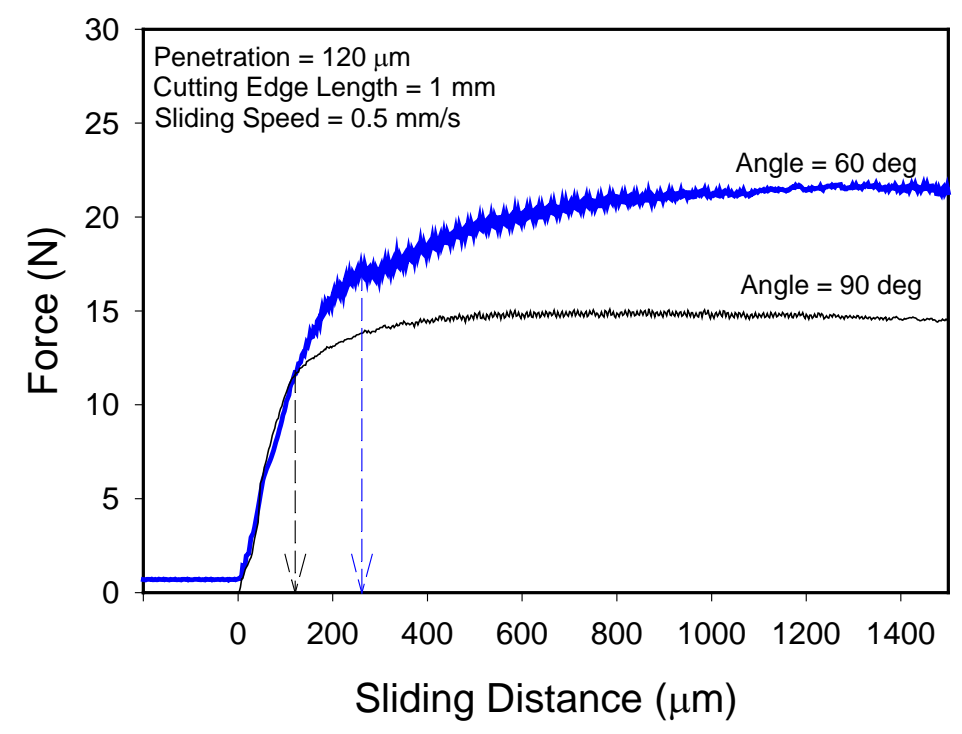

Figure 8.18 Horizontal force as a function of the sliding distance for different cutting-edge angles in a displacement-controlled cutting-edge sliding test. 


\subsection{Observations from the particle-generation results}

To validate the proposed mechanism for generation of UHMWPE particles we proposed, we also look into the morphology of the worn surface and the wear particles (the results from Chapter 7, an ASTM linear reciprocating wear process for UHMWPE particle generation). Figure 8.19 shows an example of SEM photos of the UHMWPE worn surface, the single surface feature and generated particles. Shear deformation of UHMWPE was observed along the sliding direction. The width of the sliding track seen on the worn surface is consistent with the length of the cutting edge.

According to the observation above and the analysis from the previous sections, we conclude that the process proceeds follows: At the beginning of the particle generation tests, the cutting edges penetrate into the UHMWPE material under applied normal load. During the linear sliding motion, the UHMWPE material is sheared and accumulated in front of the cutting edge. The increased volume of accumulated material leads to the increased material resistant force acting on the cutting edges. Finally the cutting edges are pushed away from the surface and a wear particle is generated.

From the micrographs, it is also observed that shear deformed UHMWPE is about to be torn away to form a wear particle. The dimensions of the volume are similar to the size of the wear particles shown in Figure 8.19. Figure 8.20 shows some other examples of higher magnified SEM micrographs of UHMWPE wear particles. The straight-line indent mark seen on the photos indicates the contact between the cutting edge and UHMWPE material. From the irregular wear particle edges, it is 
suggested that UHMWPE material breaks under a tensile stress when the cutting edge is pushed away from the surface in the sliding process.

Single surface feature

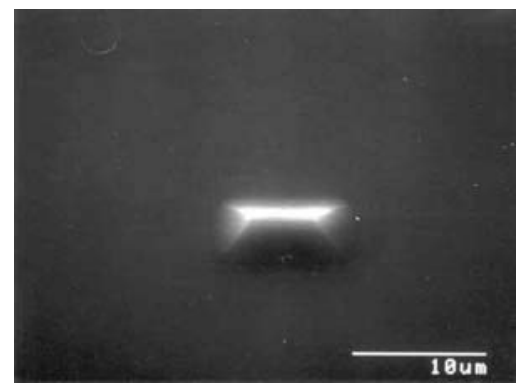

UHMWPE Worn Surface
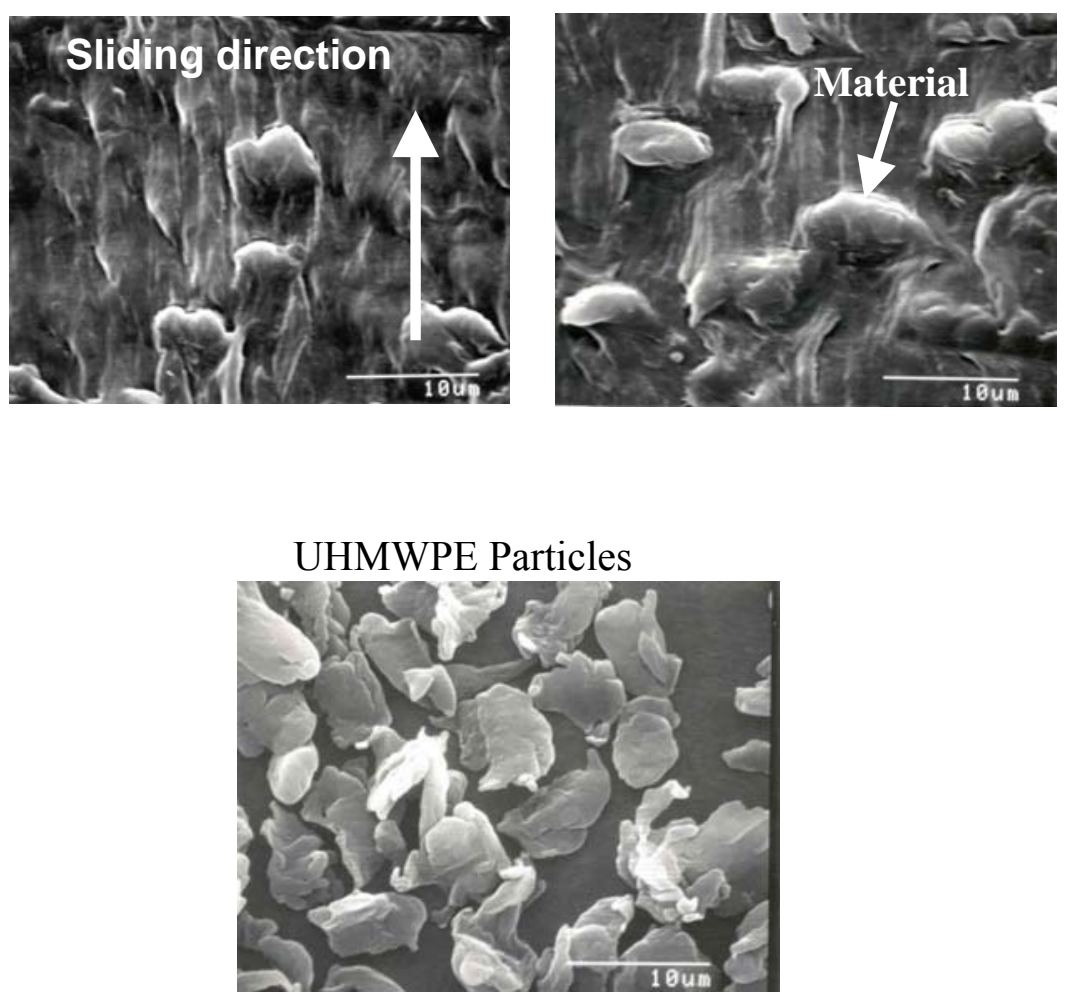

Figure 8.19 SEM micrographs of surface cutting-edge feature, UHMWPE worn surface and generated UHMWPE particles. 


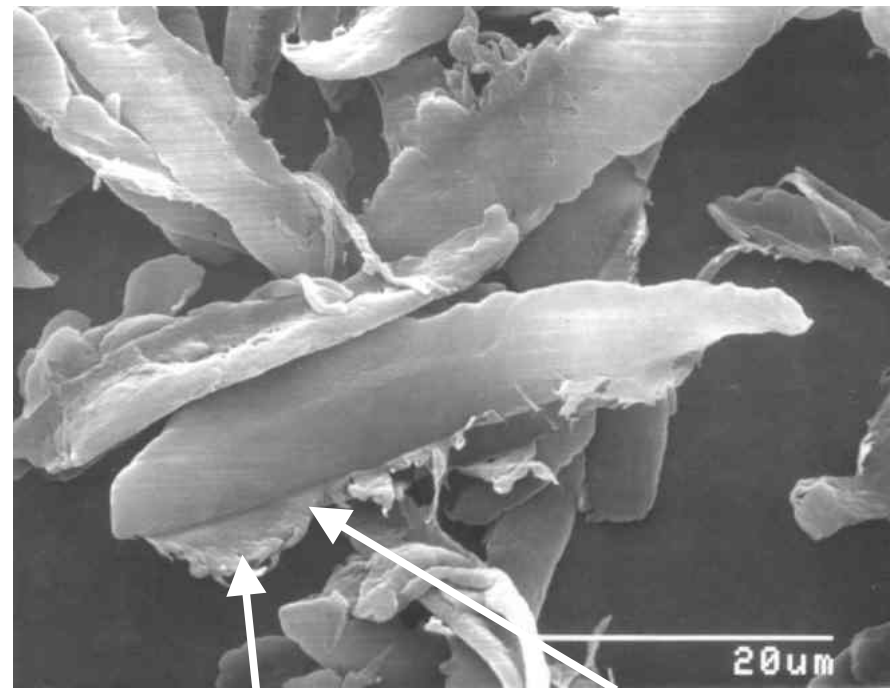

Irregular edges

Straight line indent mark

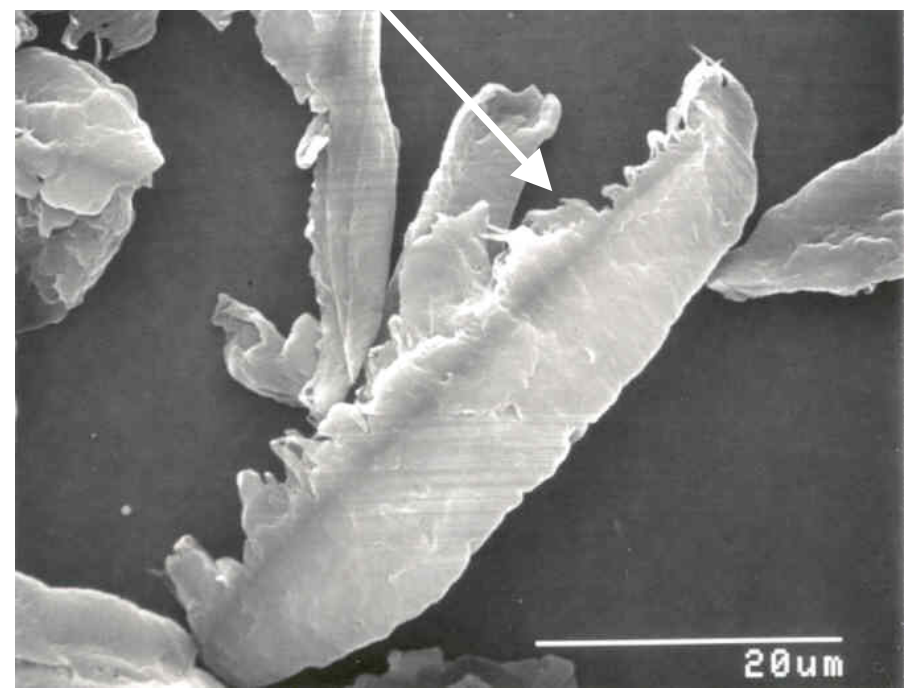

Figure 8.20 UHMWPE wear particles with irregular edges and straight line indent marks made by cutting edges. 


\subsection{Conclusions}

From the model experiments with constant load, we simulated the sliding process of the cutting edge in situ. Results of the video analysis and analysis of the reacting forces of the process provided direct evidence of the micro-cutting process of particle generation. Also based on the data for the reacting force, the effects of cuttingedge angle and penetration depth were studied. Results further confirm that a larger penetration depth leads to a longer sliding distance or a larger particle volume as suggested in Chapter 8. Shear-induced molecular orientation makes the UHMWPE surface layer more brittle under multiple passes by the cutting edges. We performed further model experiments with constant sliding depth to measure the displaced volume of UHMWPE. We also obtained the specific energy required for UHMWPE deformation during the sliding process.

The mechanism of particle generation with surface textures has been examined and supported by the model experiments. However, the quantitative relationship between the cutting feature dimensions and the particle generation is still not clear. We will attempt to model the penetration and sliding processes from first principles to quantify the relationship. In this way, we may be able to predict the particle size. 


\section{CHAPTER 9}

\section{Modeling of Particle Generation}

\subsection{Introduction}

The mechanism of UHMWPE particle generation by a textured surface has been elucidated in Chapter 8. In this chapter, we will attempt to distill our understanding into a mathematical model to predict particle size and shape with a given surface-texture dimensions and mechanical loading conditions.

We will attempt to model the particle-generation process based on first principles in two steps: (1) penetration of the cutting edge, and (2) lateral sliding of the cutting edge. The penetration depth of a wedge-shaped cutting edge is based on force balance. Predictions of the penetration depth will be made under purely plastic and elastic-plastic assumptions. We will further determine the correction factor to predict the penetration depth for viscoelastic UHMWPE material. A mathematical equation will be developed to predict the penetration depth with a given surface-feature geometry and applied loads.

During the sliding of the cutting edges, displaced volume of UHMWPE leads to the material resistant force acting on the cutting edges. The resolved component force in the vertical direction lifts up the cutting edge. The penetration depth then decreases as the tip travels further. The balance of forces between the normal load and the lift-up force contributed from the material resistance is then finally reached. We 
are going to calculate the displaced volume of UHMWPE during the sliding process. The volume is projected to be the volume of generated particles. Finally a contact mechanics model will be developed to determine the shear volume (displaced volume) of UHMWPE with given surface texture geometry and mechanical loading condition.

A correlation model based on the actual particle generation process will also be established based on the results of UHMWPE-particle generation with microfabricated surface textures. By comparing the two models, we hope to validate the first principle model.

\subsection{Model parameters}

The variables of the model are the dimensions of the surface texture, the mechanical operation parameters, and the particle dimensions as output variables. A schematic representation of the particle-generation process is shown in Figure 9.1. The important dimensions of the surface texture include the cutting-edge feature length $\left(L_{\mathrm{c}}\right)$, the feature width $\left(W_{\mathrm{c}}\right)$, the feature height $\left(H_{\mathrm{c}}\right)$, the distance between features along the sliding direction $\left(D_{\mathrm{s}}\right)$, and the distance between adjacent features $\left(D_{\mathrm{i}}\right)$. Two mechanical operation parameters during the linear reciprocating wear process are the load applied on each cutting-edge feature $(W)$, and average sliding speed $(V)$. Based on the sheet-like morphology generated from this process, the particle is characterized as the particle length $\left(L_{\mathrm{p}}\right)$, the particle width $\left(W_{\mathrm{p}}\right)$, the particle thickness $\left(T_{\mathrm{p}}\right)$, and the particle volume $\left(V_{\mathrm{p}}\right)$ is estimated by the product of these three dimensions. 


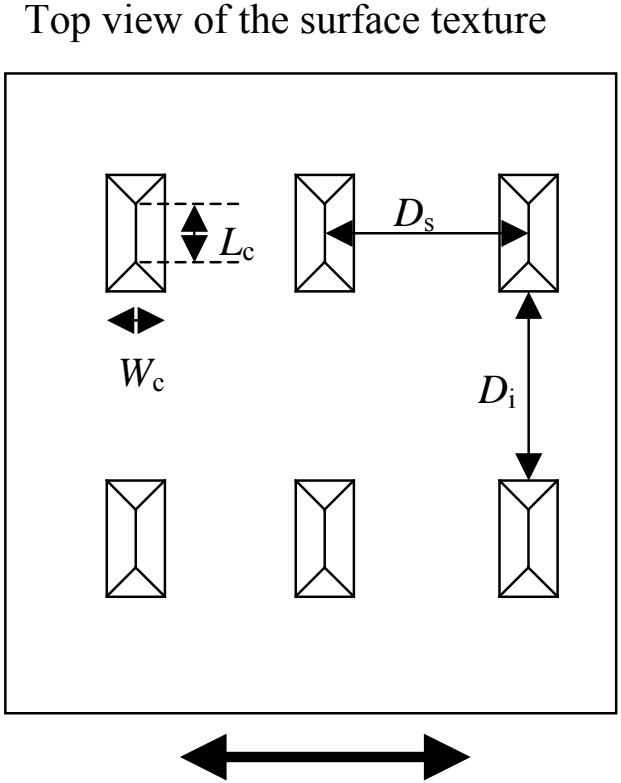

Sliding direction

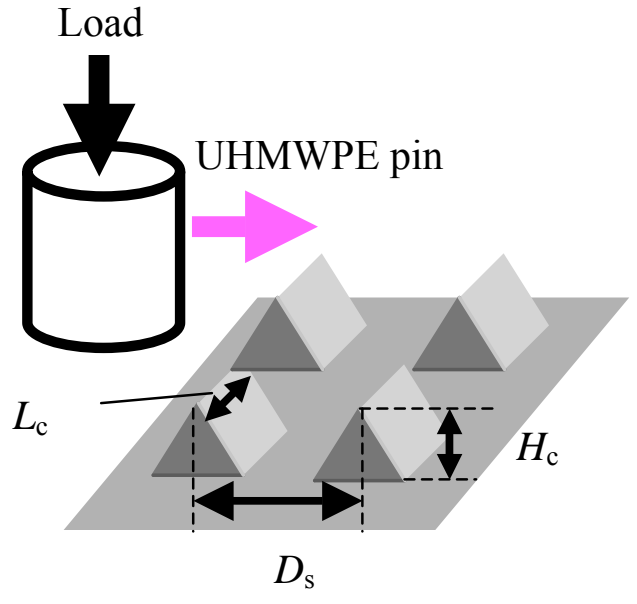

Particle length

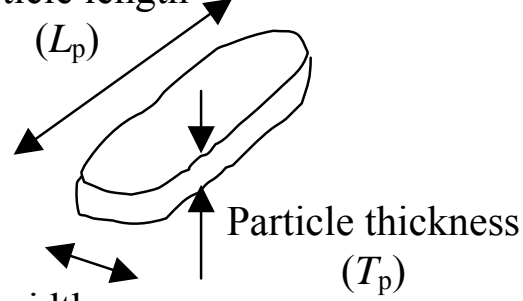

Particle width

$\left(W_{\mathrm{p}}\right)$

$L_{c}$ : cutting-edge length

$H_{\mathrm{c}}$ : cutting-edge height

$W_{\mathrm{c}}$ : cutting-edge width

$D_{\mathrm{s}}:$ pitch in the sliding direction

$D_{\mathrm{i}}$ : distance between adjacent features

Figure 9.1 Illustrations of significant variables for the particle-generation process. 


\subsection{Contact mechanics modeling}

\subsubsection{Penetration of the cutting edge}

The penetration process of the cutting edge is discussed as a wedge indentation problem in this section. A schematic representation is shown in Figure 9.2. The key parameters shown in the figure include the cutting-edge length $\left(L_{\mathrm{c}}\right)$, half angle of the cutting edge $(\theta)$, average normal load on each feature $(W)$ and the penetration depth $\left(H_{\mathrm{p}}\right)$. Theoretical calculations of the penetration depth of a wedge tip with purely plastic and elastic-plastic assumptions have been carried out previously [111-113]. We are going to derive the equations from a force balance.

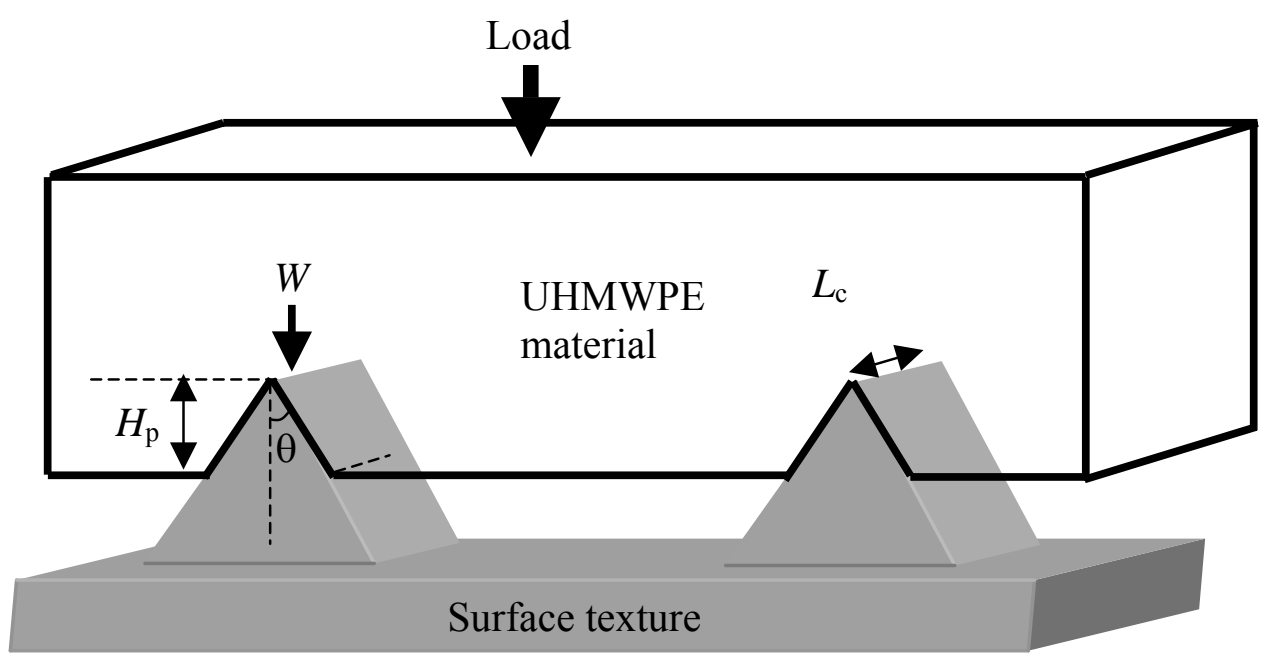

Figure 9.2 Schematic of the penetration process of the cutting edges. 
As shown in Figure 9.2, the average normal load acting on each cutting edge is $W$. The contact length after the indentation process is $2 \cdot H_{\mathrm{p}} \cdot \tan \theta$. The average contact pressure of applied to the material can be calculated by:

$$
\text { Average contact pressure } p_{\mathrm{pen}}=\frac{W}{2 \cdot L_{\mathrm{c}} \cdot H_{\mathrm{p}} \cdot \tan \theta} \text {. }
$$

With the perfect plastic deformation assumption, no deformation will occur until the yield stress is reached, after which plastic flow occurs. The relationship between average contact pressure and the yield stress has the following relationship [111]:

$$
p_{\text {pen }}=C_{\mathrm{p}} \cdot Y,
$$

where $C_{p}$ is a coefficient for different geometry of the indenter. For a wedge shaped indenter, the $C_{\mathrm{p}}$ is a function of $\theta$ and is described as $[111,112]$

$$
C_{\mathrm{p}}=\lambda[2.57-0.023(90-\theta)],
$$

where $\lambda=2 / \sqrt{3}$ by the von Mises yield criterion, and where $\theta$ is expressed in degrees. By balancing the pressure in the $\mathrm{z}$ direction, we obtain:

$$
\frac{W}{2 \cdot L_{\mathrm{c}} \cdot H_{\mathrm{p}} \cdot \tan \theta}=C_{\mathrm{p}} \cdot Y
$$

Thus the penetration depth $\left(H_{\mathrm{p}, \mathrm{p}}\right)$ predicted by the purely plastic hypothesis can be expressed as

$$
H_{p, p}=\frac{W}{2 \cdot\left(C_{p} \cdot Y\right) \cdot L_{c} \cdot \tan \theta},
$$

where $Y$ is the yield strength of the material. 
With an elastic-plastic assumption of the material property, a certain amount of deformation will be recovered after the applied stress is released. Johnson [113] has derived that $H / Y$ ( $H$ : hardness, $Y$ : yield strength) is a function of penetration depth and $E / Y$ (E: elastic modulus). By representing hardness by the normal force and the size of deformation site after penetration, the predicted penetration depth with elastic-plastic assumption can be described as:

$$
H_{\mathrm{p}, \mathrm{p}}=\frac{W}{2 \cdot\left(C_{\mathrm{ep}} \cdot Y\right) \cdot L_{\mathrm{c}} \cdot \tan \theta},
$$

where $C_{\mathrm{ep}}$ is a function of material property and penetration edge geometry. For a wedge geometry, $C_{\mathrm{ep}}$ is derived by Johnson [113] as the following:

$$
C_{\mathrm{ep}}=\frac{1}{\sqrt{3}}\left[1+\ln \left(\frac{4}{3 \pi} \frac{E}{Y} \tan \left(\frac{\pi}{2}-\theta\right)\right],\right. \text { for wedge geometry. }
$$

For example, the $C_{\mathrm{ep}}$ value of a $90^{\circ}$ cutting-edge angle $\left(\theta=45^{\circ}\right)$ wedge feature is 2.49 , and the $C_{\text {ep }}$ value of a $60^{\circ}$ cutting-edge angle $\left(\theta=30^{\circ}\right)$ wedge feature is 2.80 for the indentation of UHMWPE material.

To discuss the effect from viscoelasticity, we shall obtain the experimental penetration-depth data for UHMWPE. Wedge indenters $\left(90^{\circ}\right.$ and $60^{\circ}$ with $2 \mathrm{~mm}$ edge length) were applied to the indentation tests. The normal load was applied and holds for $15 \mathrm{~s}$. Given sufficient time $(60 \mathrm{~min})$ after the indentation tests, profiles of the indentation marks were measured by profilometry. The various normal loads were tested and the measured penetration depths were compared with the elastic-plastic calculation in Figure 9.3. 

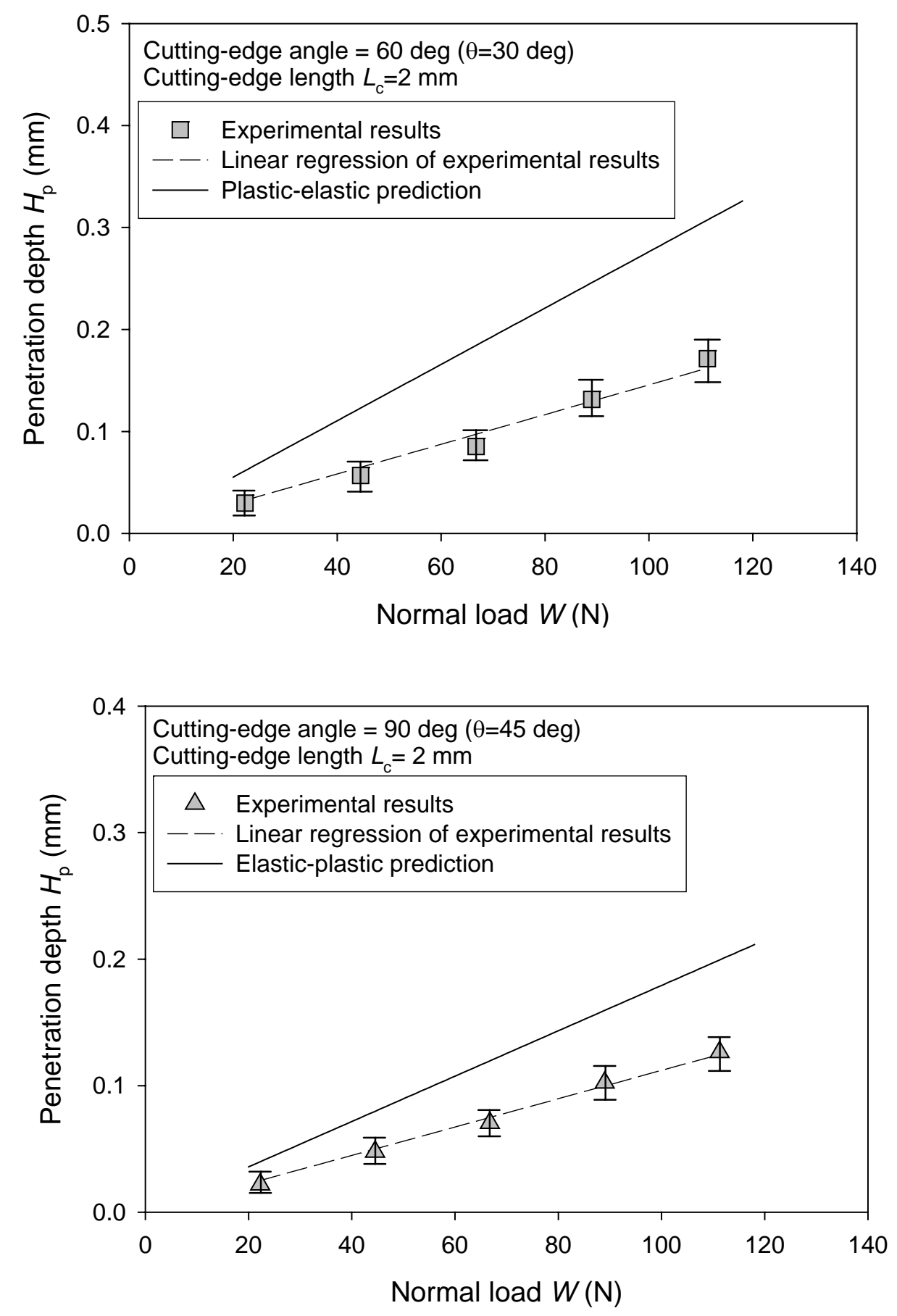

Figure 9.3 Comparison of the experimental data of wedge indentation in UHMWPE with elastic-plastic calculation results. 
We can also represent the experimental results by the following equation that is similar to that for the plastic and elastic-plastic calculations:

$$
H_{\mathrm{p}}=\frac{W}{2 \cdot\left(C_{\mathrm{v}} \cdot Y\right) \cdot L_{\mathrm{c}} \cdot \tan \theta} \quad,
$$

where $C_{\mathrm{v}}$ is the coefficient for the viscoelastic UHMWPE from the experimental results. By comparing the elastic-plastic prediction results with the linear regression curve of the experimental results, we obtain

$$
C_{\mathrm{v}}=1.75 \cdot C_{\mathrm{ep}},
$$

where $C_{\mathrm{ep}}$ is the coefficient for the elastic-plastic model. The penetration depth can be then predicted from the above equation after the viscoelastic properties are taken into account. It means the ratio of penetration depth predicted from the elastic-plastic assumption to the measured penetration depth is $1.75 \pm 0.12$ (mean \pm standard deviation). The ratio we obtained from the nano-indentation test in section 8.2 is 1.39 . These results are reasonably close.

Figure 9.3 shows that the prediction from the calculation with elastic-plastic assumption is larger than the experimental measurements. It is suggested that the difference arises from the viscoelasticity of UHMWPE for the following possible reasons: (1) Recovery of material deformation: After the indentation process, removal of the applied stress results in the gradually recovery of material deformation. The viscoelastic recovery has been measured from a nano-indentaion test in Chapter 8. (2) Creep behavior during the indentation: For a viscoelastic material, the material deformation is a function of the strain rate. Under a high strain rate, the time allowed 
for material deformation is short and thus the final material deformation is smaller than for the case with low strain rate under the same applied stress, and (3) Strain hardening of the material: During the indentation process, the plastic deformation brings the strain hardening of the material. It leads to an increased material resistant during the indentation process and a smaller penetration depth.

The viscoelastic recovery of the material in the cutting-edge penetration process has a dramatic influence on the final penetration depth. We conclude that the

final penetration depth can be estimated from equation (9.8). The final penetration depth during the indentation process can be obtained.

\subsubsection{Sliding process of the cutting edge}

During the sliding process, a horizontal displacement is applied to the cutting edge under a constant normal load and the material is ploughed. A schematic representation of the process is shown in Figure 9.4. The introduction of the horizontal sliding process unloads the left-hand face of the cutting edge. Further vertical deformation takes place by the cutting edge sliding further deeper in the sliding process. UHMWPE material displaced by the cutting edge was accumulated in front of the edge. The resultant force from the displaced volume acts on the cutting edge as an encountered force to the normal load. The net normal load is decreased and it leads to the decrease of the penetration depth. During the subsequent sliding process, the displaced volume for unit sliding distance is decreased. More volume is accumulated in front of the cutting edge with a decreasing rate of increase of displaced volume. It is 
an iterative process between increase of resistant force by increased displaced volume and decrease of penetration depth by the increase of material resistant force. Finally the cutting edge is pushed away from the UHMWPE material and the displaced volume of UHMWPE is projected to be the volume of the generated particle.
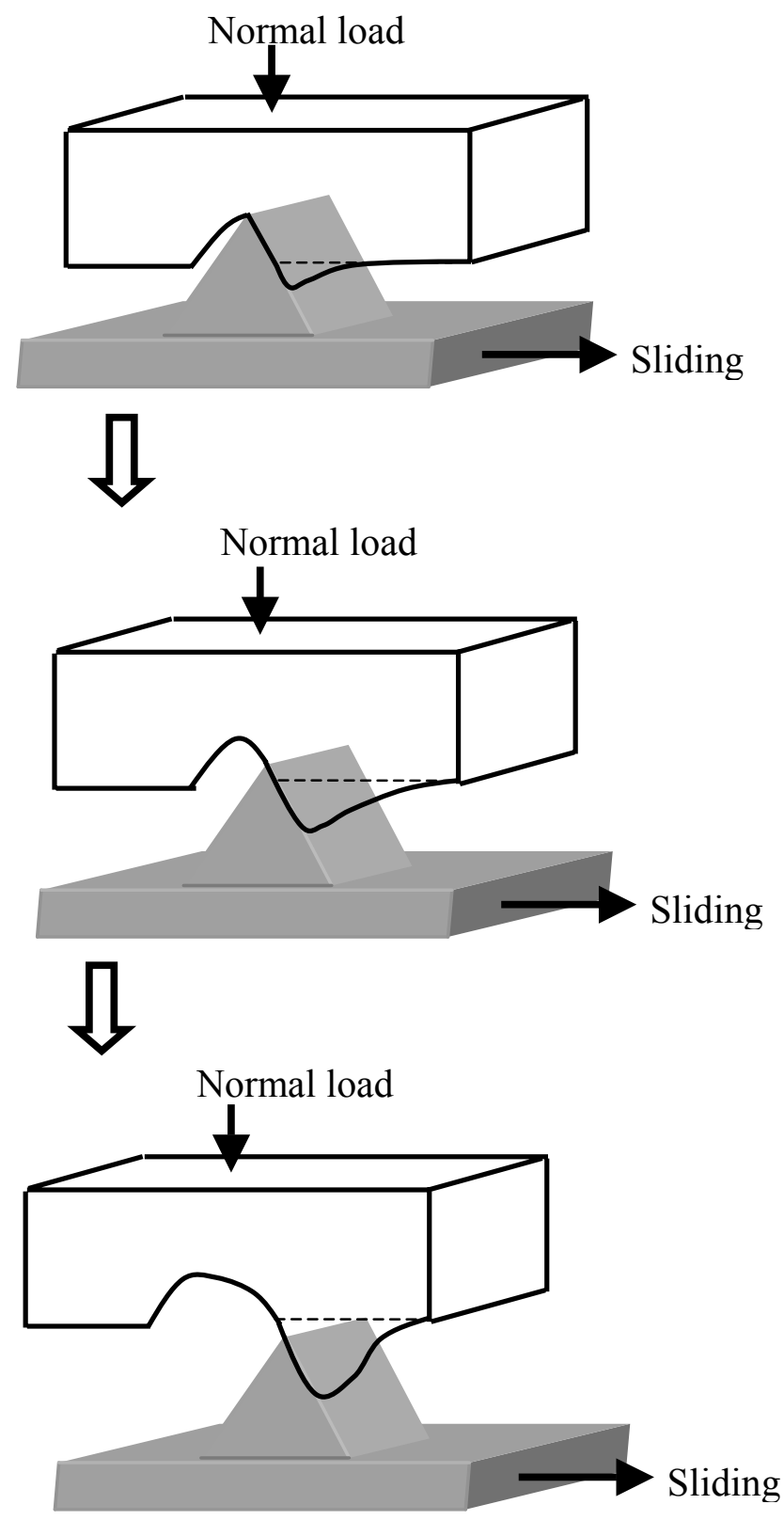

Figure 9.4 Schematic representation of the sliding process of the cutting edge. 
We are going to calculate the projected volume of the particle based on various dimensions of cutting edges and mechanical loading conditions. Figure 9.5 shows a flow chart of the calculation procedure. With known $L_{\mathfrak{c}}, W_{\mathfrak{c}}, H_{\mathrm{c}}$, and $\theta$, the penetration depth can be calculated. During the sliding process, one face of the cutting edge was unloaded due to the horizontal sliding motion. The supporting area of the cutting edge is half of the area in an indentation process. Equation (9.8) is then modified as equation (9.10) to calculate the penetration depth during the sliding process:

$$
H_{\mathrm{pc}}=\frac{W}{\left(C_{\mathrm{v}} \cdot Y\right) \cdot L_{\mathrm{c}} \cdot \tan \theta} \text {. }
$$

If the calculated penetration depth is larger than the feature height $H_{c}$, the real penetration depth is equal to the feature height. The minimum load $W_{\mathrm{m}}$ needed to reach the penetration depth $H_{\mathrm{c}}$ is then back calculated:

$$
W_{\mathrm{m}}=H_{\mathrm{c}} \cdot\left(C_{\mathrm{v}} \cdot Y\right) \cdot L_{\mathrm{c}} \cdot \tan \theta .
$$

With a small increment of sliding distance, we can calculate the displaced volume by the product of cutting edge length, penetration depth and the increment of sliding distance. With known quantity of displaced volume $\left(V_{\mathrm{d}}\right)$, we can estimate the material resistant force. From the simple model experiment described in chapter 8 , we have obtained the material resistant force per unit volume of UHMWPE is equal to $m=1.2 \cdot 10^{-6} \mathrm{~N} /(\mu \mathrm{m})^{3}$ (estimated slope of the plot shown in Figure 8.15) 


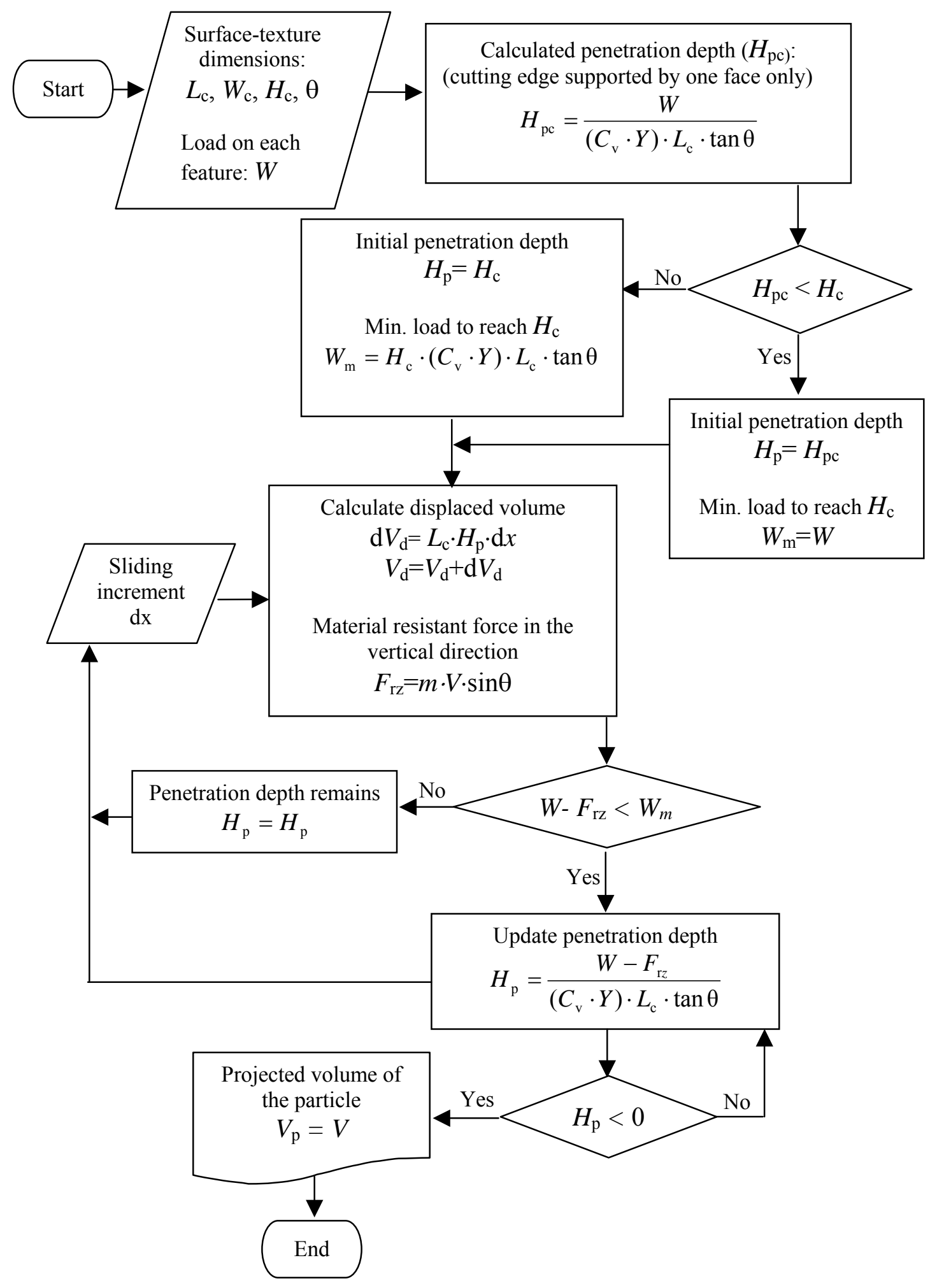

Figure 9.5 A flow chart of calculation of the sliding process of the cutting edge. 
Then we can calculate the resolved resistant force in the vertical direction from the displaced volume of UHMWPE:

$$
F_{\mathrm{rz}}=F_{\mathrm{r}} \cdot \sin \theta=m \cdot V_{\mathrm{d}} \cdot \sin \theta .
$$

Obviously, the cutting-edge angle plays an important role at distributing the material resistant force into the vertical direction as shown in Figure 9.6. With the same displaced volume, a larger force is distributed to the vertical direction for a larger cutting-edge angle. The penetration depth was then updated by the new normal load in the equation (9.13):

$$
H_{\mathrm{p}}=\frac{W-F_{\mathrm{rz}}}{\left(C_{\mathrm{v}} \cdot Y\right) \cdot L_{\mathrm{c}} \cdot \tan \theta} .
$$

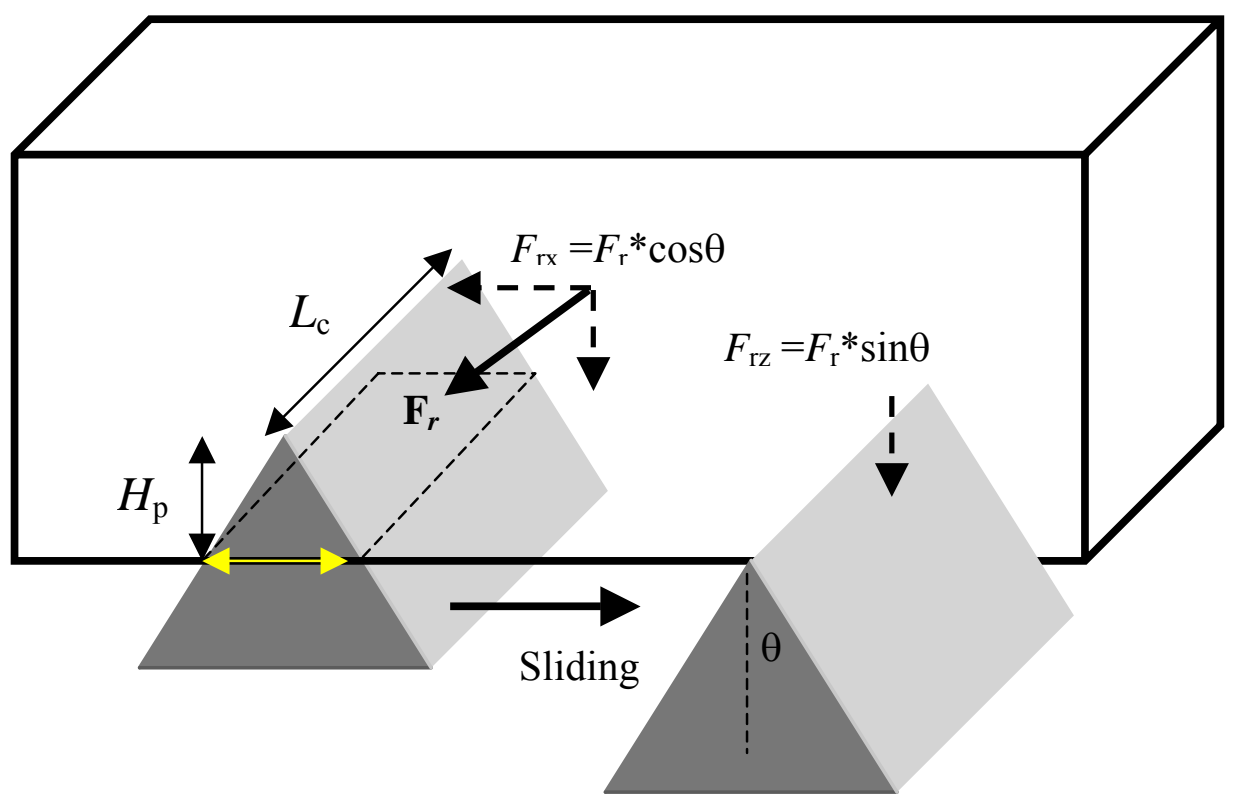

Figure 9.6 A schematic representation of reacting forces in a cuttingedge sliding process. 
For the case with initial penetration depth equal to the feature height, there exists excess load between the cutting edge and UHMWPE material. The cutting edge needs to displace a larger volume of UHMWPE during the sliding process in order to generate sufficient resistant force to push away the cutting edge from the material surface. This factor is also taken into account in our model.

After we update the penetration depth for a small increment of sliding distance, the new penetration depth was then used to calculate the displaced volume in the next increment of sliding. Assuming incompressibility of the material, the accumulated displaced volume of UHMWPE was determined by adding up the displaced volume from each sliding increment. The iteration process was carried out until the penetration depth reaches zero. It indicates the cutting edge was pushed away from the UHMWPE surface. The total displaced volume during the process is projected to the particle volume.

The process was written in a MATLAB program as shown in Appendix D. In the next section, we are going to compare the experiment results with the calculation from this model. We are also going to discuss the effect of surface-texture dimensions and mechanical loading conditions on the generated particles from the model predictions. 


\subsubsection{Validation of the model}

In this section, we are going to calculate the predicted particle volume with given surface-texture dimensions and mechanical loading conditions. For the experimental results, the particle volume was estimated by the product of particle length, particle width and particle thickness from the SEM observations. The predictions by the model will be compared to the experimental results. The effects of normal load, feature height, and cutting-edge angle will also be discussed.

We have generated UHMWPE particles with surface texture A-3 which was listed in Figure 7.3 with two different load conditions. The dimensions of the surface texture are $L_{\mathrm{c}}=7.0 \mu \mathrm{m}, W_{\mathrm{c}}=5.0 \mu \mathrm{m}$, and $H_{\mathrm{c}}=3.0 \mu \mathrm{m}$. The tests were run under an average sliding speed of $57.2 \mathrm{~mm} / \mathrm{s}$. The normal loads applied were $192 \mathrm{~N}$ and $96 \mathrm{~N}$, which corresponds to $0.1174 \mathrm{~N}$ and $0.0587 \mathrm{~N}$ distributed on each feature on the surface. Figure 9.7 shows the comparison of the model predictions and the experimental results. Both results show that increase of load lead to a larger particle volume. From our calculations, it indicated that the minimum load needed $\left(W_{\mathrm{m}}\right)$ on each feature to reach the penetration depth equal to the feature height is only 0.0017 $\mathrm{N}$. The loads we applied were far larger than this value. It means the cutting edge was pushed to the end of the surface. Most of the material resistant force developed was used to encounter the excess load on the surface. A linear relationship is observed from the prediction from the model.

However, the model predicted particle volume is larger than the ones from experimental results. One possible reason is that the particle length is about $10 \%$ to 15 
$\%$ smaller than the cutting-edge length. In the model, we have assumed that the particle length is equal to the cutting-edge length. The viscoelasticity, leading to the shrinkage of the particle dimension after the particle is released from the bulk UHMWPE, can explain some of the difference between predicted and the experimental results. On the other hand, the multiple surface features on the surface texture may re-processing the generated particles and further reduce the particle size. With the same feature density of the surface texture, there exists higher possibility for a larger particle to be re-processed in the micro-cutting process. In the model, we assumeed a linear relationship between reacting forces and displaced volume during the sliding process of the cutting edge. However, a nonlinearity was also observed from Figure 8.15 toward a larger displaced volume. It is probably why we observed a larger difference between the model prediction and the experimental results for the larger particle

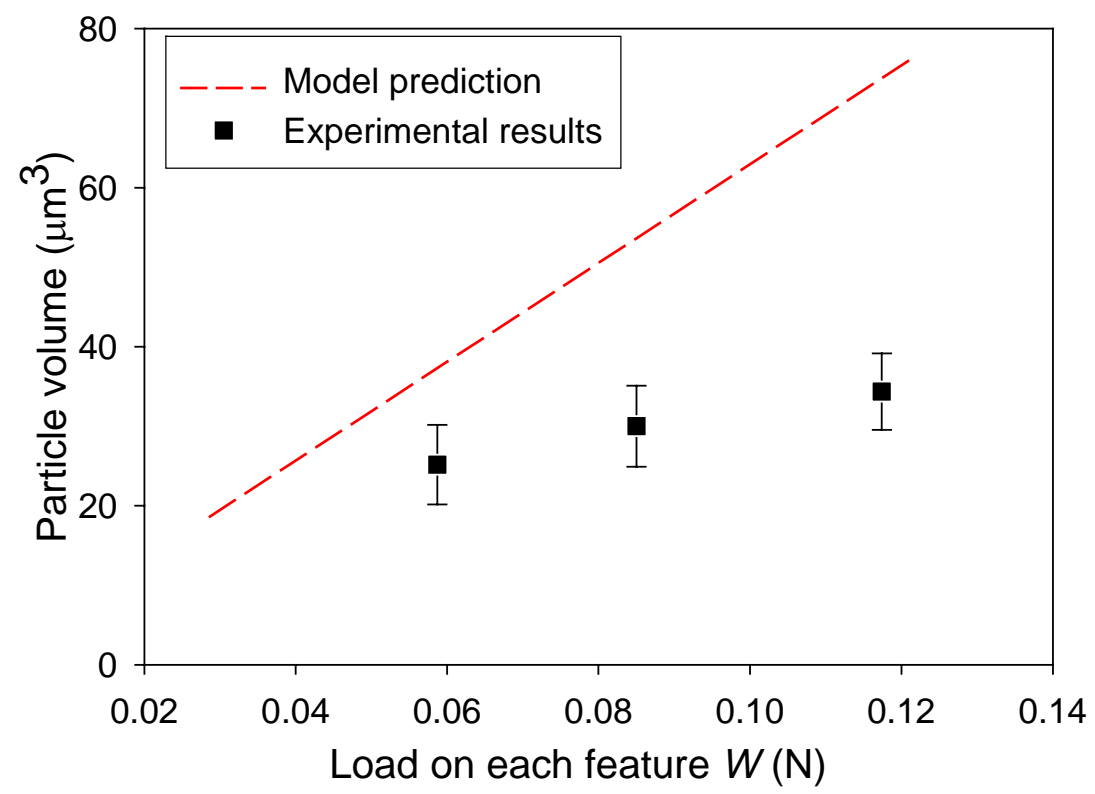

Figure 9.7 Effect of normal load from model predictions and experimental results. 
Surface textures A-2 with different feature heights were applied in the particlegeneration process. The dimensions of the surface texture are $L_{\mathrm{c}}=15.0 \mu \mathrm{m}, W_{\mathrm{c}}=5.0$ $\mu \mathrm{m}$, and two different feature heights with $H_{\mathrm{c}}=3.4 \mu \mathrm{m}$ and $2.8 \mu \mathrm{m}$. The tests were run under an average sliding speed of $57.2 \mathrm{~m} / \mathrm{s}$ and a normal load of $96 \mathrm{~N}$ (load applied on each feature $W=0.0587 \mathrm{~N}$ ). The micrographs of the particles have been shown in Figure 7.6. The comparison of the model prediction and the experimental results are shown in Figure 9.8. A larger feature height leads to a larger particle volume observed from the model predictions and experimental results. The applied load is also sufficient to push the cutting edge to the end of the surface. A larger feature height means a larger initial penetration depth at the beginning of the sliding process. Obviously a larger penetration depth leads to a longer sliding distance and a larger particle volume.

The results again show that the particle volume predicted by the model is larger than the experimental results. From Figure 9.8 it indicates that the difference between the model prediction and experimental results is smaller for a larger particle. The trend is opposite from the previous case. Probably it is more difficult for the surface texture with a larger feature height to entrap the generated particles between the cutting edges and UHMWPE surface. Thus less number of larger particles were reprocessed so the experimental results are closer to the model predictions which are based on a single cutting-edge process. 


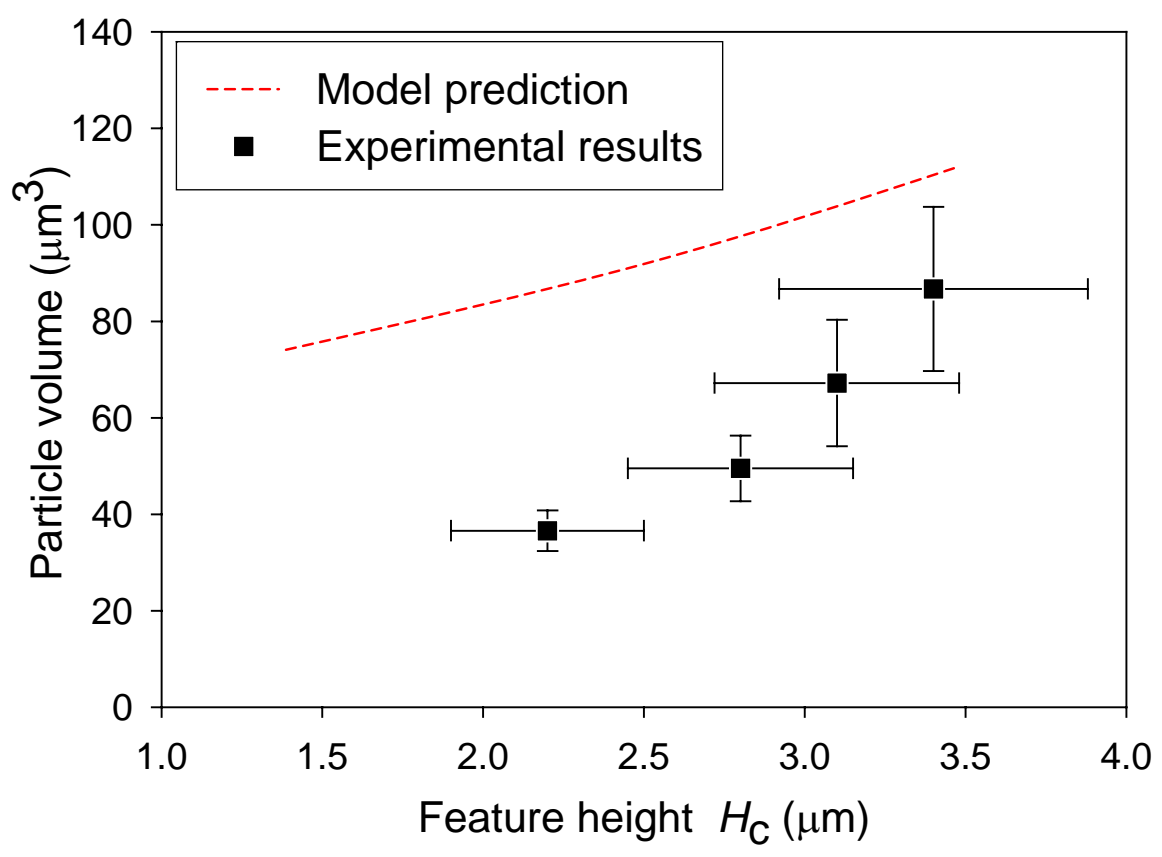

Figure 9.8 Effect of feature height from model predictions and experimental results.

There is lack of experimental data with different cutting-edge angles while maintaining the same feature height of the surface texture. We used the feature length and feature height dimensions from surface texture A-2 $\left(L_{c}=15.0 \mu \mathrm{m}, H_{\mathrm{c}}=2.8 \mu \mathrm{m}\right)$. By artificially changing the feature width in the model, we can evaluate the effect of cutting-edge angle. A normal load of $96 \mathrm{~N}$ (load applied on each feature $W=0.0587$ N) was also used in the model. Figure 9.9 shows the particle volume versus different cutting-edge angles from the model calculations. A sharper cutting edge (smaller cutting-edge angle) results in a larger particle volume. With the same penetration depth, the sharper cutting edge leads to a longer sliding distance because a smaller material resistant force is distributed to the vertical direction to push away the cutting 
edge. Obviously the predictions from the model are consistent with the theoretical approach of the particle-generation mechanism.

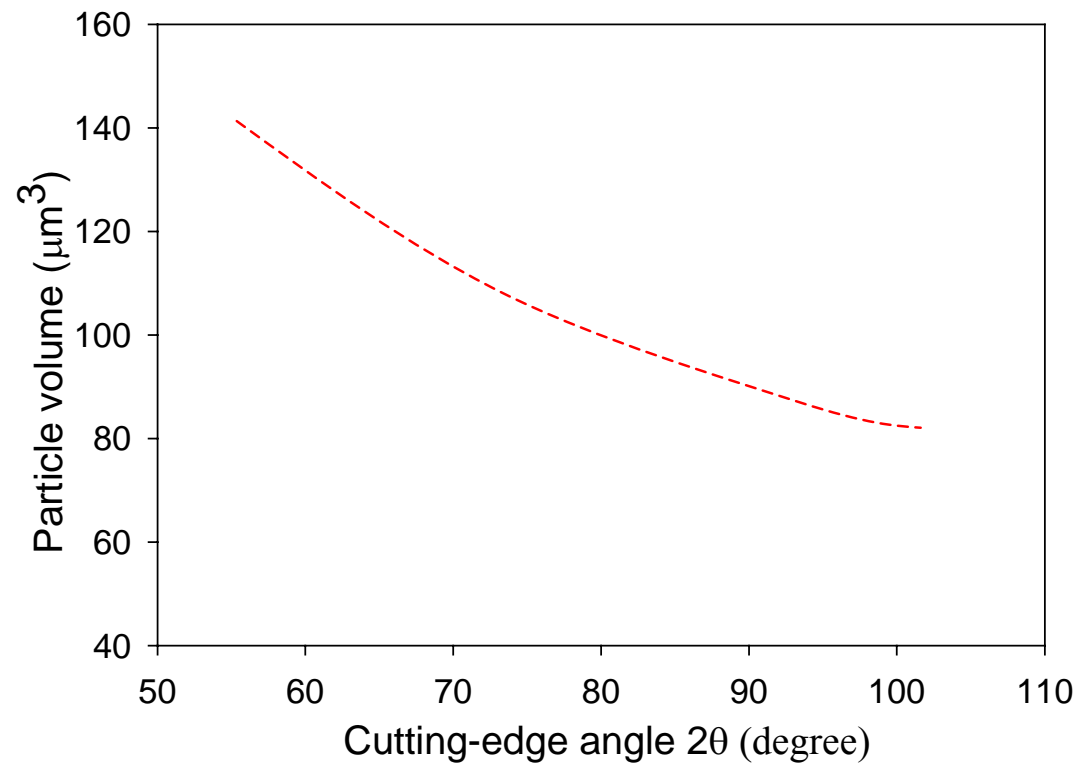

Figure 9.9 Effect of cutting-edge angle from model calculations.

To sum up, we have successfully predicted the effects of surface textures on particle volume qualitatively. This achievement enables us to predict the particle volume from a first principle approach. This model can assist on the design of surface textures in order to generated UHMWPE particles with desired size and shape. The results of the prediction are close to the experimental results of UHMWPE particle generated. However, the model predicts a larger particle volume. In order to further quantitatively predict the particle volume precisely, the model should be further tightened to reflect the facts of multiple cutting edges and limited penetration depth in the real particle-generation process. 


\subsection{Correlation model}

\subsubsection{Effect of cutting-edge length}

As shown in Figure 9.1, the direction of the cutting-edge length is perpendicular to the scratching direction. It is observed experimentally that the particle length is close to but a little smaller than the cutting edge. Measured values of particle dimensions have a relative standard uncertainty of $\pm 8 \%$, and measured dimensions of cutting edges have a relative standard uncertainty of $\pm 5 \%$. SEM photos of different cutting-edge lengths versus different particle lengths are compared in Figure 9.10. By linear regression of the plot in Figure 9.11, a linear relationship is obtained:

$$
L_{\mathrm{p}}=0.78 \cdot L_{\mathrm{c}} .
$$

The correlation coefficient of the regression $r^{2}$ is 0.997 .

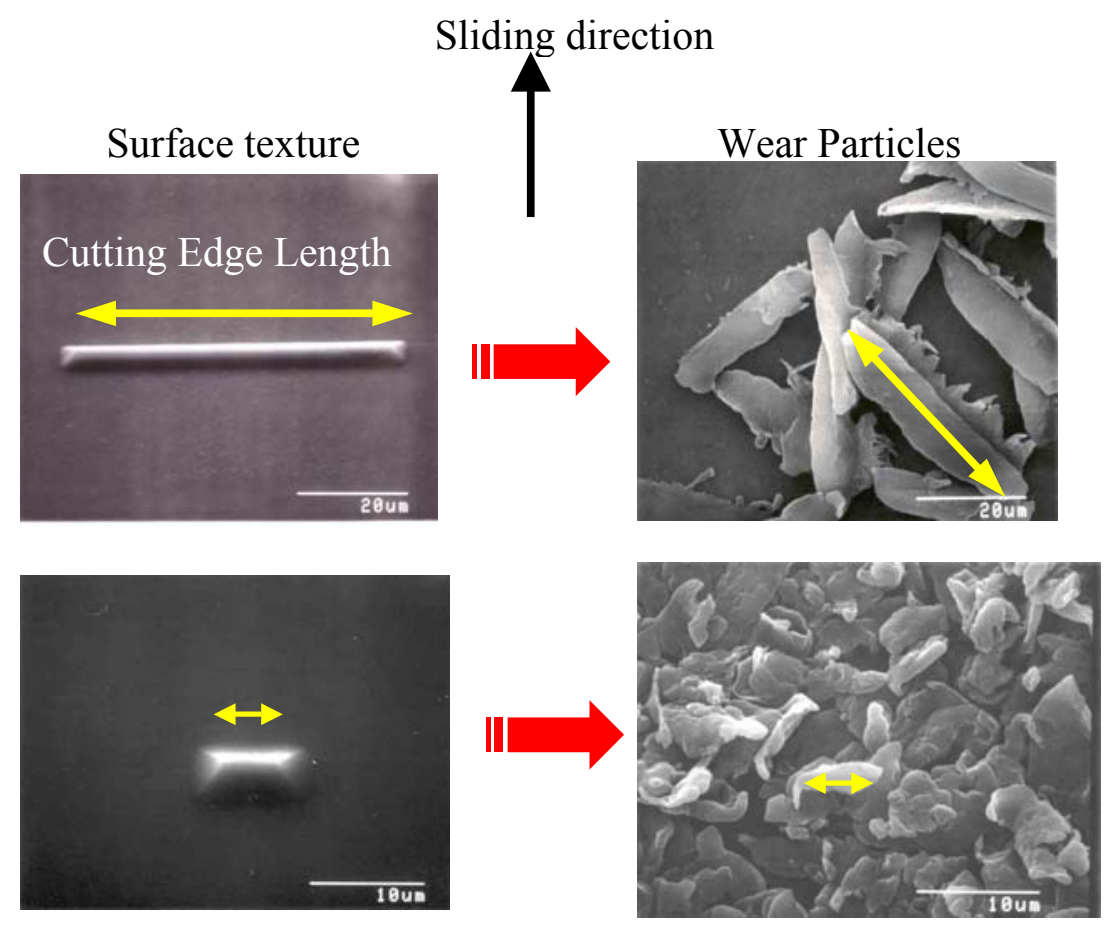

Figure 9.10 Comparison of cutting-edge length with wear-particle length. 


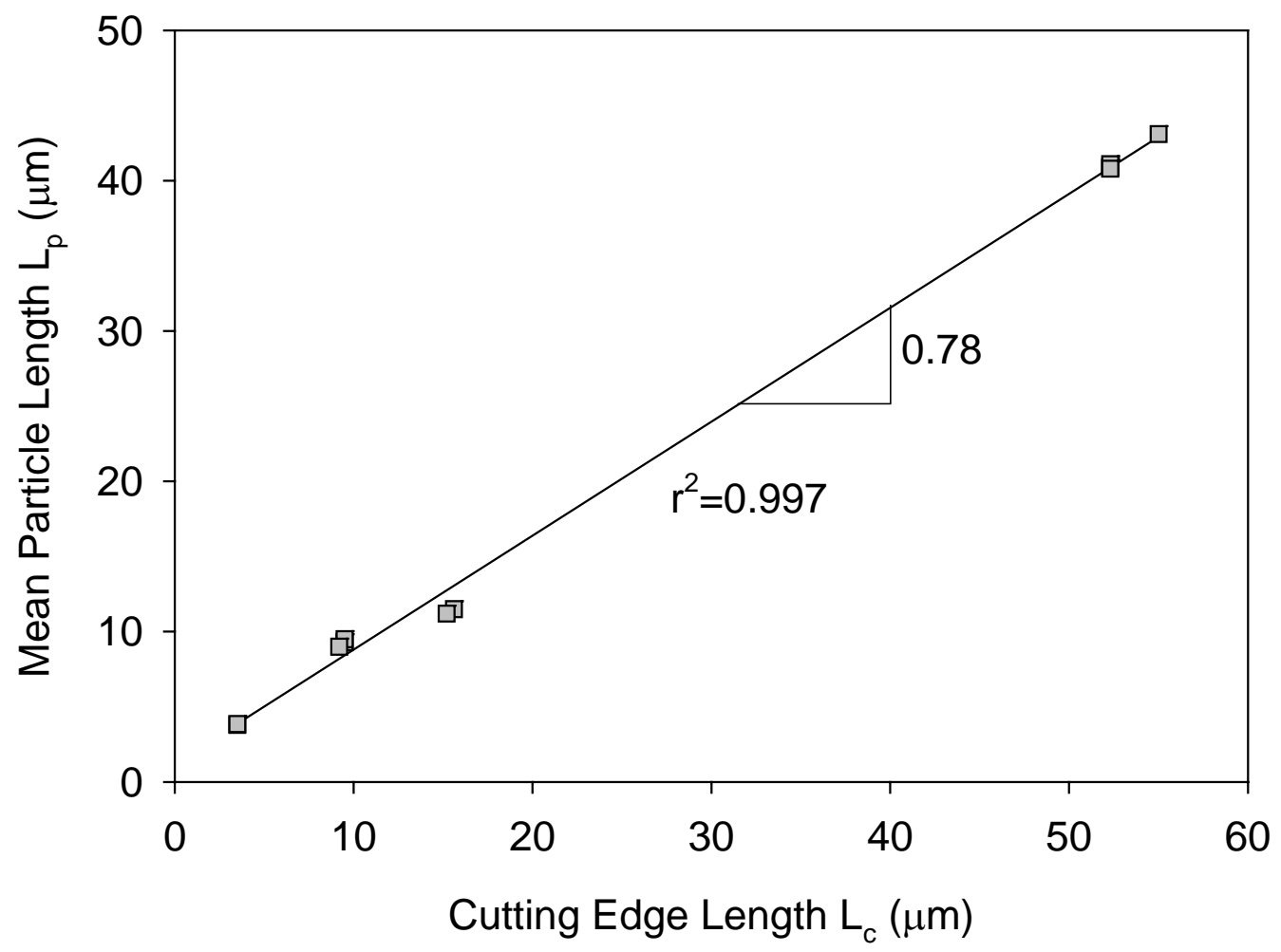

Figure 9.11 Plot of particle length versus cutting-edge length. 


\subsubsection{Effect of penetration depth}

The variables that affect the penetration depth include (a) geometrical dimensions of cutting edges: cutting edge length $\left(L_{\mathrm{c}}\right)$, cutting edge height $\left(H_{\mathrm{c}}\right)$, cutting-edge angle, and (b) mechanical condition: applied load for each cutting-edge feature $(W)$. The penetration depth with purely plastic and elastic-plastic assumptions concerning the material properties can be calculated. The detailed derivation and calculation procedures have been presented in the previous section. However, in our case, the maximum penetration depth is limited by the feature height $\left(H_{\mathrm{c}}\right)$. When the calculated penetration depth exceeds the feature height, penetration depth $\left(H_{\mathrm{p}}\right)$ is equal to cutting-edge height $\left(H_{\mathrm{c}}\right)$.

Previous research has shown the effect of the cutting penetration depth on the thickness of formation chips during the machining process [110]. By applying the same principle to the generation process here, theoretically the particle thickness should be proportional to penetration depth of the cutting edge. The ratio between the two values depends on the geometry of the cutting edges. Figure 9.12 shows a plot of particle thickness versus different penetration depths from our experimental results obeying a relationship:

$$
T_{\mathrm{p}} \propto H_{\mathrm{p}}
$$

We also have plotted the particle width versus penetration depth in Figure 9.13. A linear relationship between them is also obtained:

$$
W_{\mathrm{p}} \propto H_{\mathrm{p}}
$$




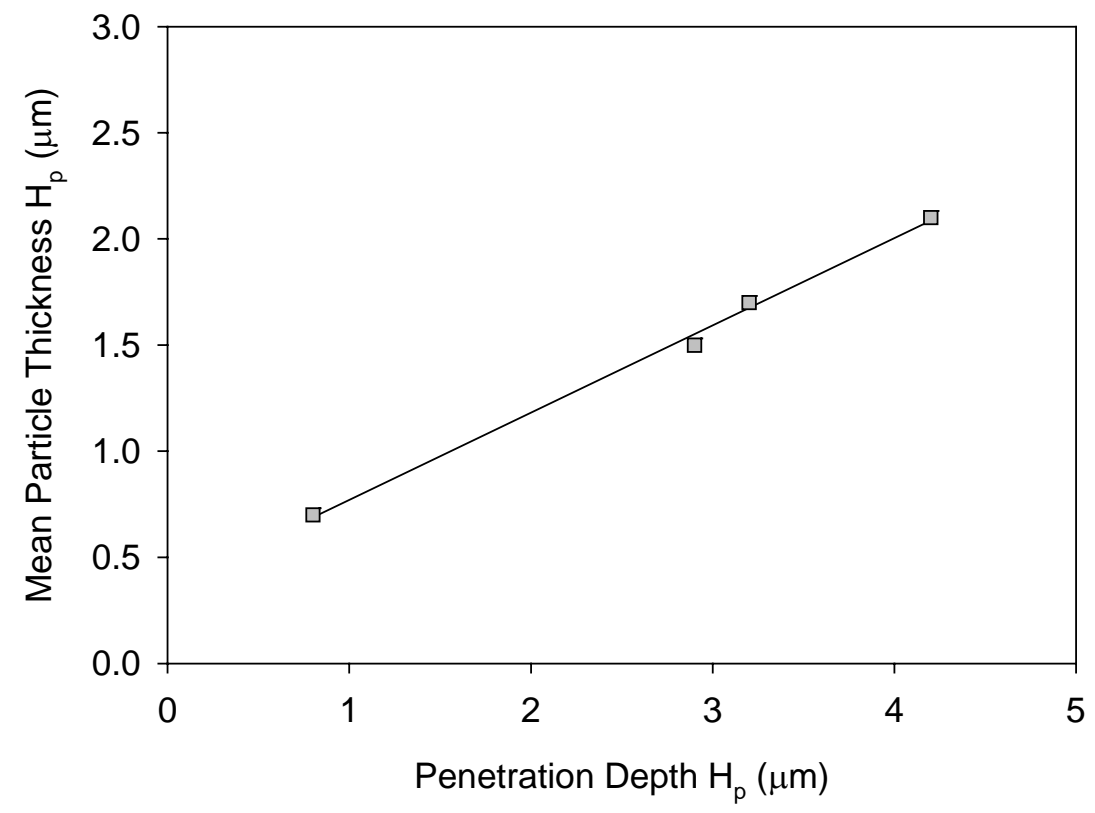

Figure 9.12 Plot of particle thickness versus penetration depth.

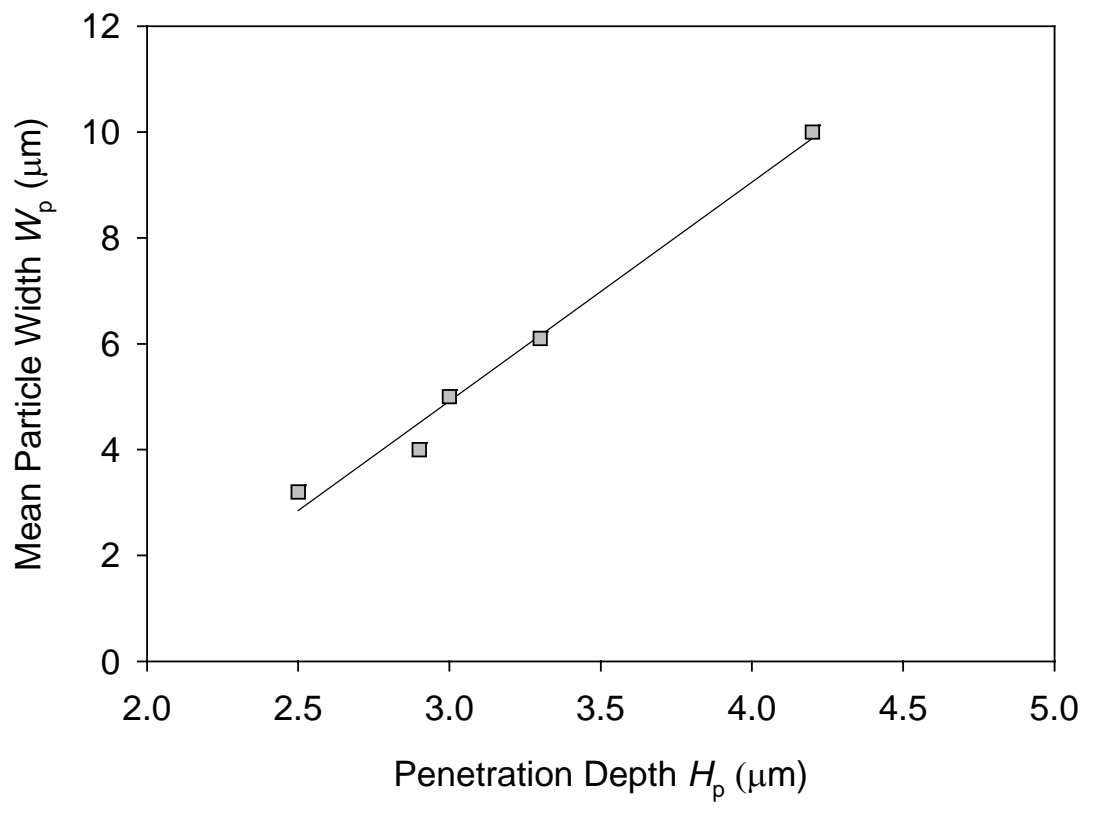

Figure 9.13 Plot of particle width versus penetration depth. 


\subsubsection{Effect of load}

Figure 9.14 is a plot of effect of load on particle volume. Tests have been done on the same surface texture with different applied load. The particle volume increases with increased applied normal load. By plotting the load $W$ vs. the particle volume $V_{\mathrm{p}}$ on a log-log scale, we obtain:

$$
V_{\mathrm{p}} \propto W^{0.6}
$$

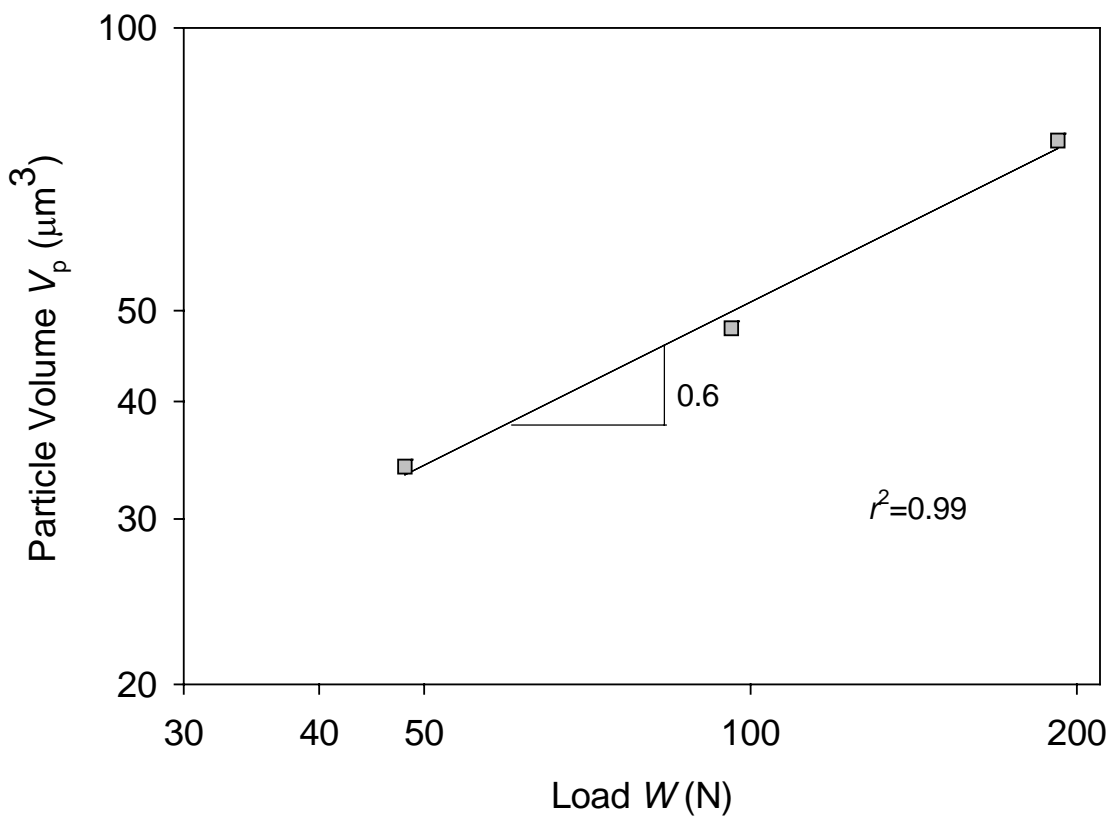

Figure 9.14 Plot of particle volume versus load on a log-log scale. 


\subsubsection{Effect of sliding speed}

The same surface texture was tested under different sliding speeds. By plotting the particle size $V_{\mathrm{p}}$ versus average sliding speed $S p$ on a log-log scale in Figure 9.15, we obtain:

$$
V_{\mathrm{p}} \propto \frac{1}{S p^{0.6}}
$$

A lower speed leads to a larger particle size. The sliding speed influences the residence time of the cutting edge during the cutting process, especially for a viscoelastic material. A lower sliding speed allows more stress relaxation in the sliding process. It leads to a longer sliding distance before the particle is generated.

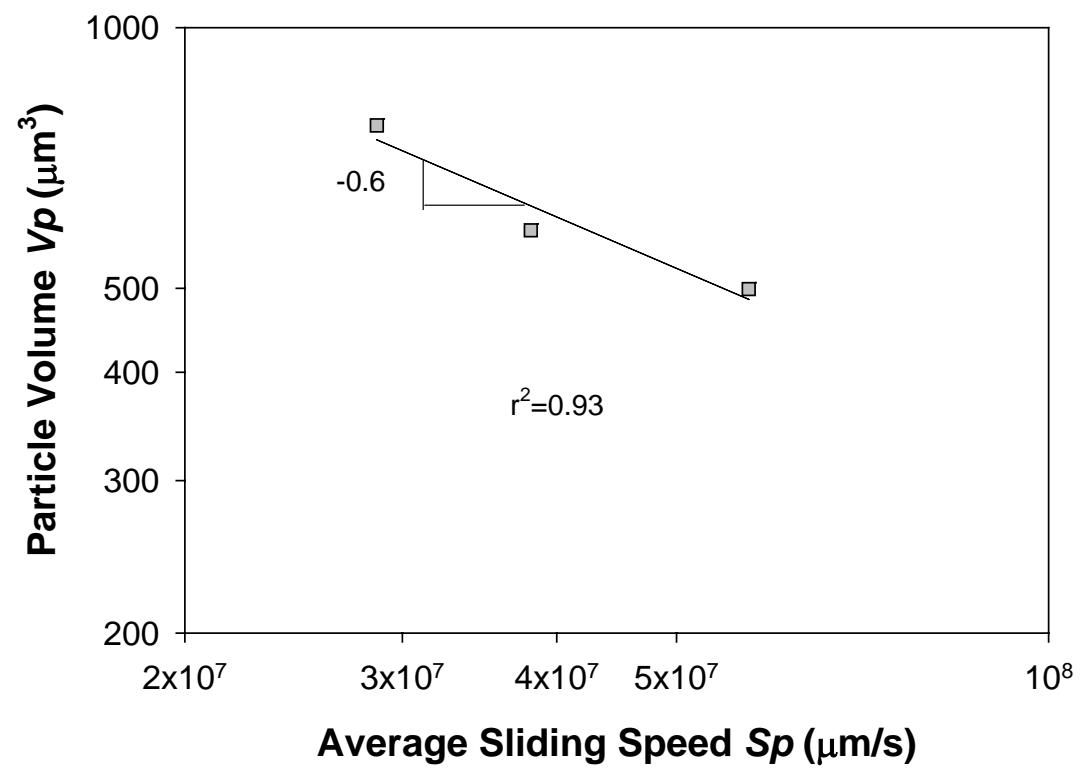

Figure 9.15 Plot of particle volume versus sliding speed on a log-log scale. 


\subsubsection{Comparison with experimental results}

The relationship between particle length, width, thickness, surface texture geometry, and mechanical conditions are described by equations (9.14)-(9.18). For the micro-cutting process, the particle volume can be estimated from the product of particle length, width and thickness. Thus the following relationship is obtained:

$$
V_{\mathrm{p}} \propto \frac{L_{\mathrm{c}} \cdot H_{\mathrm{p}}^{2} \cdot W^{0.6}}{S p^{0.6}}
$$

The particle volume $\left(V_{\mathrm{p}}\right)$ is plotted against $\frac{L_{\mathrm{c}} \cdot H_{\mathrm{p}}{ }^{2} \cdot W^{0.6}}{S p^{0.6}}$ in Figure 9.16. A linear relationship is found and the $95 \%$ confidence level shows that the experimental data can be predicted well by the following equation:

$$
\left.V_{\mathrm{p}}=39.5 \frac{L_{\mathrm{c}} \cdot H_{\mathrm{p}}{ }^{2} \cdot W^{0.6}}{S p^{0.6}} \text { (unit: } \mu \mathrm{m}, \mathrm{N}, \mathrm{s}\right)
$$

\subsection{Summary}

Two approaches have been attempted to model the UHMWPE-particle generation with surface textures. Contact mechanics model calculated the penetration depth and then calculated the displaced volume of UHMWPE iteratively from a forcebalance basis. The results of the model predictions are close to the experimental results of the particle generation. Correlation model correlate the variables in the UHMWPE-particle generation process to a mathematical equation. Both model indicates the same trend of effects of surface-texture geometry and mechanical conditions on the volume of particles. 


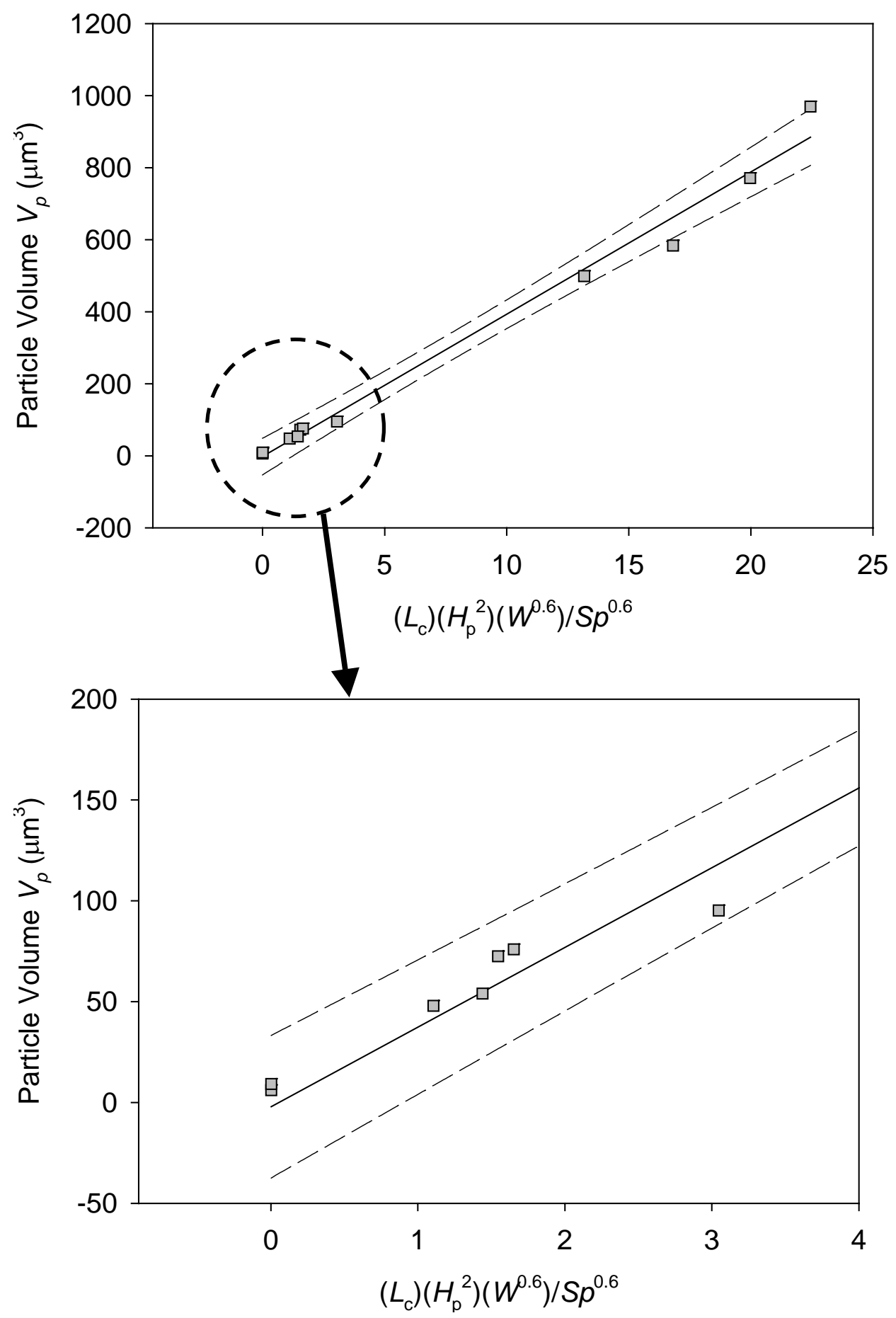

Figure 9.16 Comparison of the correlation model with the experimental results. 


\section{CHAPTER 10}

\section{PARTicle Characterization by ANALYZing The Phagocytosis of Particles}

\subsection{Introduction}

In artificial joint systems, UHMWPE wear particles generated during the life of the replacement have been associated with osteolysis and prosthetic loosening. The morphology of the wear particles varies according to different articulating geometry, material pairs, and mechanical conditions. From the viewpoints of phagocytosis of wear particles, the mechanisms of particles induced bioactivities will vary according to different particle size and shape [32]. In order to identify the critical parameters of particles for induced osteolysis, characterization of particles from the viewpoint of phagocytosis of particles is necessary.

In this chapter, we will explain how the human body attempts to digest UHMWPE wear particles. Phagocytosis of particles is a key phenomenon in the process. We will investigate the phagocytic capacity which is the quantity of particles that one macrophage cell can phagocytose from a biophysical point of view ${ }^{1}$. From this, a foundation will be built for the subsequent chapter of bioactivity testing results and their relationship with particle dimensions and shapes.

\footnotetext{
${ }^{1}$ The phagocytosis of particles only represents one perspective of the complicated processes for UHMWPE particle induced osteolysis. The purpose is to analyze the effects of particle size and shape on bioactivity from an engineering point of view.
} 


\subsection{Basic particle parameters}

\subsubsection{Descriptors for particle size}

After the image analysis of the particles (described in section 4.4.2), the dimensions of the generated particles including particle length, width and thickness can be estimated from the SEM photo of the particle as shown in Figure 10.1. The particle size can be represented by the volume of the particle. Based on the sheet-like geometry of the UHMWPE particles, the volume of the particle can be calculated as the product of the particle length, width and thickness:

$$
V_{\mathrm{p}} \approx L_{\mathrm{p}} \cdot W_{\mathrm{p}} \cdot T_{\mathrm{p}}
$$

From the viewpoints of reaction between particles and cells, the surface area of the particle represents the effect of particle size on the resulting outcomes [86]. For a sheet-like particle as shown in Figure 10.1, the surface area $\left(S_{\mathrm{p}}\right)$ of the particle can be estimated from equation (10.2) when the particle thickness is much smaller than the particle length and width:

$$
S_{\mathrm{p}} \approx 2 \cdot L_{\mathrm{p}} \cdot W_{\mathrm{p}}
$$

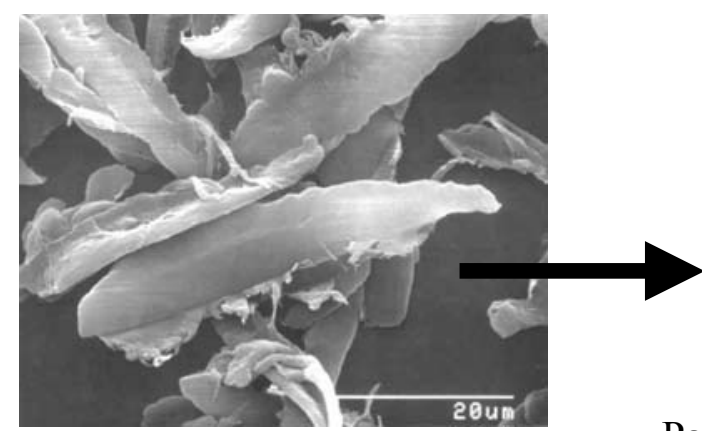

Particle length

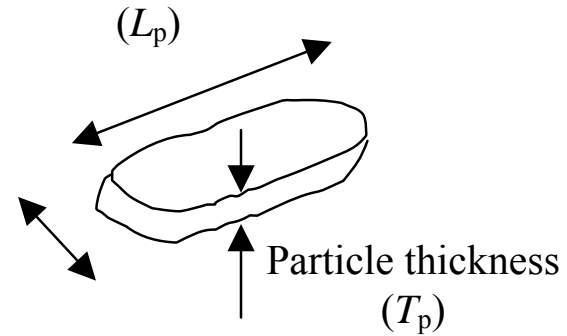

Particle width

$\left(W_{\mathrm{p}}\right)$

Figure 10.1 Estimation of particle length, particle width and particle thickness from a SEM micrograph of UHMWPE particles. 
From the viewpoints of the phagocytosis of the particles by microphage cells in bioactivity tests, the concepts of projected particle volume and the projected particle surface area are proposed here. When a particle is phagocytosed by a macrophage cell, the particle is wrapped by the membranes of the macrophage cells. The membranes of macrophage cells tend to form a spherical or ellipsoid shape after engulfing the particles. Based on this argument, we can calculate the volume and the surface area of an ellipsoid which has three axis lengths equivalent to the particle length, width and thickness as shown in Figure 10.2. We define them as the projected particle volume and projected particle surface area according to the following equations:

$$
\begin{gathered}
V_{\mathrm{pp}}=\frac{4}{3} \cdot \pi \cdot L_{\mathrm{p}} \cdot W_{\mathrm{p}} \cdot T_{\mathrm{p}}, \\
S_{\mathrm{pp}} \approx 2 \pi \cdot L_{\mathrm{p}} \cdot W_{\mathrm{p}}\left(\text { for } L_{\mathrm{p}}>>T_{\mathrm{p}}\right),
\end{gathered}
$$

where $V_{\mathrm{pp}}$ is the volume of the projected ellipsoid and the $S_{\mathrm{pp}}$ is the surface area of the projected ellipsoid.
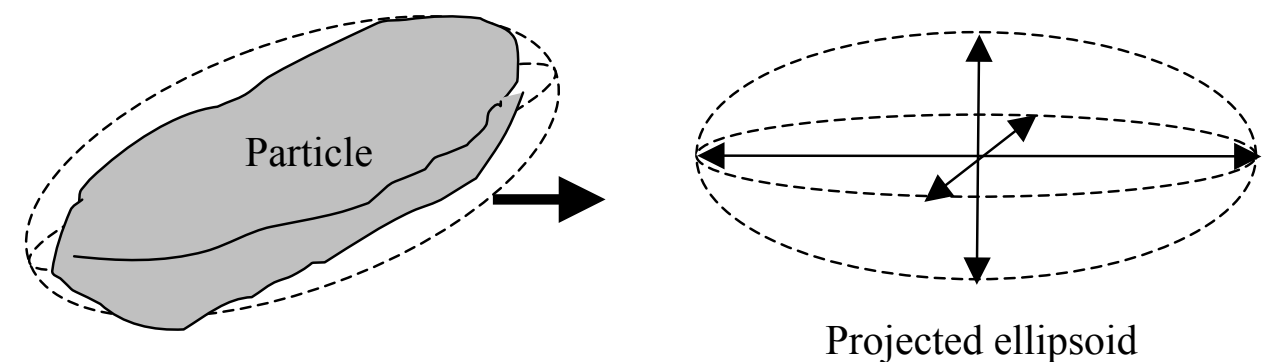

Figure 10.2 Schematic of the projected ellipsoid of a particle. 


\subsubsection{Descriptors for particle shape}

The descriptors for particle shape are used to distinguish various particle shapes quantitatively. Elongated particles and spherical-like particles have been observed for the UHMWPE wear particles from clinical studies of total joint implants. In this section, the discussion focuses on quantifying the degree of the elongation of a wear particle.

During the particle-collection process, as mentioned in Chapter 4, a vacuum filtration process was applied to collect the particles on the filter membrane surface. The sucking of the vacuum system forces the particles to lay on a two dimensional plane. Then the SEM micrographs of the particles were taken for further image analysis. Based on the understanding of the particle collection process, it is assumed that the height of the particle on the filter surface is the smallest dimension of the particle. The following analysis of the particle shape will be made on a twodimensional analysis of the SEM micrographs of the particles.

As shown in Figure 10.3, the particle length $\left(L_{\mathrm{p}}\right)$ is defined as the longest length along its axis and the particle width $\left(W_{\mathrm{p}}\right)$ is the average dimension perpendicular to the particle axis. Examples of the particle length and width for different model particles are also shown in Figure 10.3. From this basis, the following shape parameters have been defined to quantify the degree of the elongation of a particle: 

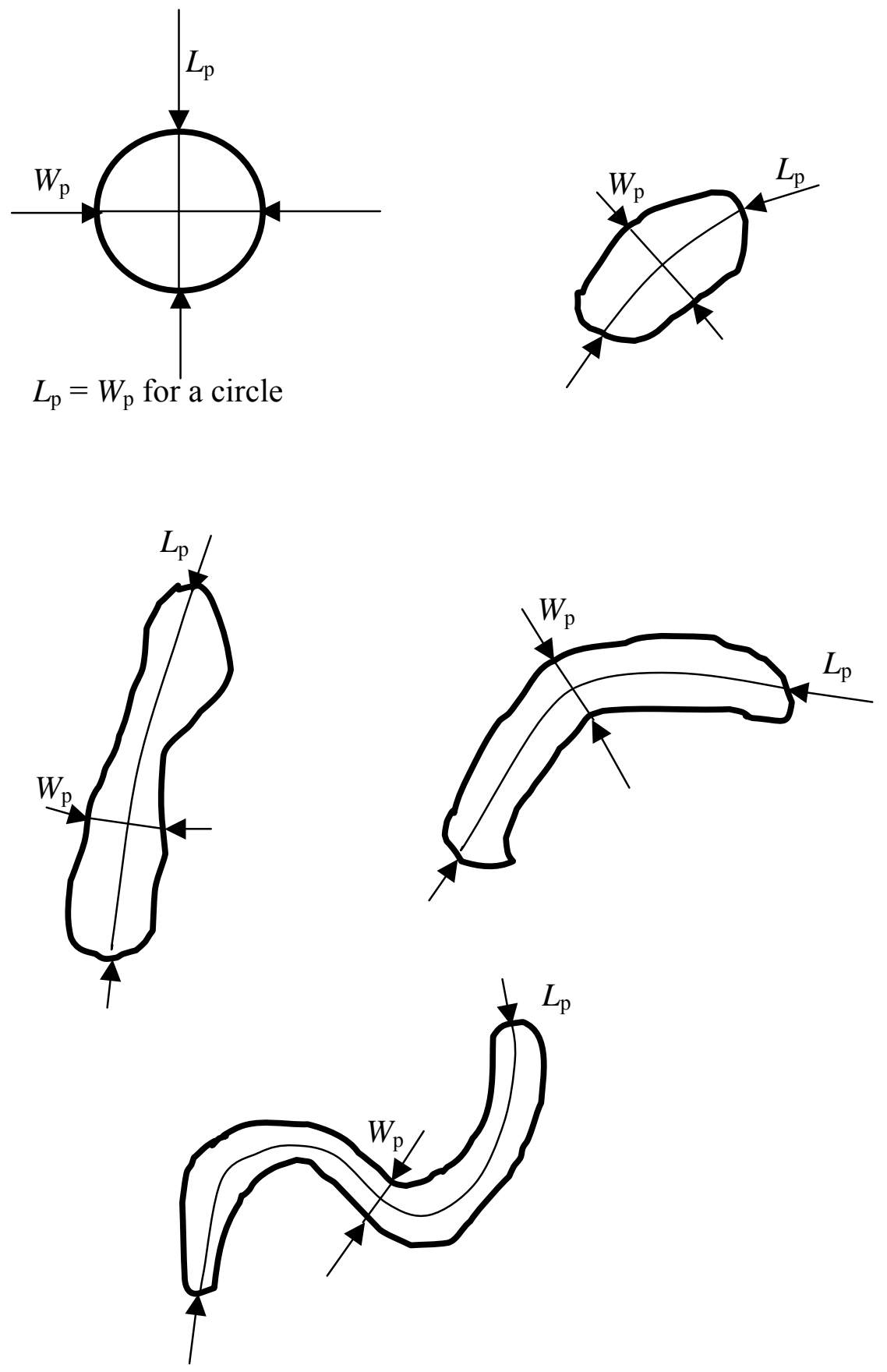

Figure 10.3 Schematic of the definition of the particle length $\left(L_{\mathrm{p}}\right)$ and the particle width $\left(W_{\mathrm{p}}\right)$ for different shapes of particles. 
(1) Aspect ratio $(A R)$ : The aspect ratio is the ratio of the particle length to the particle width. It is a measure of the elongation of a boundary profile. The larger aspect ratio, the longer is the particle.

$$
\text { Aspect } \operatorname{Ratio}(A R)=\frac{L_{p}}{W_{p}}
$$

(2) Roundness: The roundness is a measure of how closely a particle resembles a circle based on the area of the two-dimension particle image. The roundness is sensitive to the elongation of a boundary profile. The roundness is equal to unity for a circle and is smaller than unity for any other shape. The roundness can be calculated from equation (10.6):

$$
\text { Roundness }=\sqrt{\frac{4 \cdot A_{\mathrm{p}}}{\pi \cdot\left(L_{\mathrm{p}}\right)^{2}}}
$$

where $A_{\mathrm{p}}$ is the area of the two-dimension particle image.

(3) Form factor: The form factor measures the deviation of a particle from a circle. The baseline is the perimeter of a circle instead of the area of a circle defined in the roundness. The form factor is sensitive to both the ruggedness and elongation of a profile. The form factor is equal to unity for a circle and is less for any other shape. The form factor can be calculated from equation (10.7):

$$
\text { Form factor }=\sqrt{\frac{4 \cdot \pi \cdot A_{\mathrm{p}}}{(\text { perimeter })^{2}}}
$$




\subsection{Osteolysis induced by UHMWPE particles}

Aseptic loosening has been recognized as a factor causing failure of total joint replacements since 1960 . The basic science of bone loss surrounding the artificial joints has been investigated [78-87]. It is now generally accepted that the osteolysis is an outcome of an adverse cellular response that involved the phagocytosis of wear particles including UHMWPE debris, metal particles, bone cement particles and possible bone chips. Retrieved tissues, animal tests, and cell-culture studies have provided the approaches to understand the complexity of cellular interactions and chemical mediators involved in osteolysis. Major responsible cells in the process include macrophage, osteoblast, and osteoclast cells. The related chemical mediators that are responsible for the cellular responses and effects on bone resorption include tumor necrosis factor-alpha (TNF- $\alpha$ ), interleukin-1 (IL-1), interleukin-6 (IL-6), and prostaglandin $\mathrm{E}_{2}$ (PGE-2). Figure 10.4 shows a schematic of possible mechanisms for particles-induced osteolysis.

Studies of retrieved wear particles have provided information of size range and shape of particles according to the types of joint replacement, various material components, and material parts undergoing different sterilization processes. Some studies have suggested that particles of phagocytosable sizes stimulate higher bioactivity and that higher doses elicit more response (often up to a saturation level) [81]. Variation of particles-induced bioactivity is usually established by comparison of the amounts of released chemical mediators or calcium concentration. Different particle sizes have been compared based on the same particle numbers, particle 
volumes, particle-surface areas and their ratio to cell numbers $[81,86]$. However, an indicator that links the degree of phaogyctosis with the particles-induced bioactivity is still lacking. A theoretical discussion and calculation of the physical aspects of the phagocytosis process is attempted in this chapter. Reviewing various results of cellculture studies validates the calculation of the phagocytosis process.

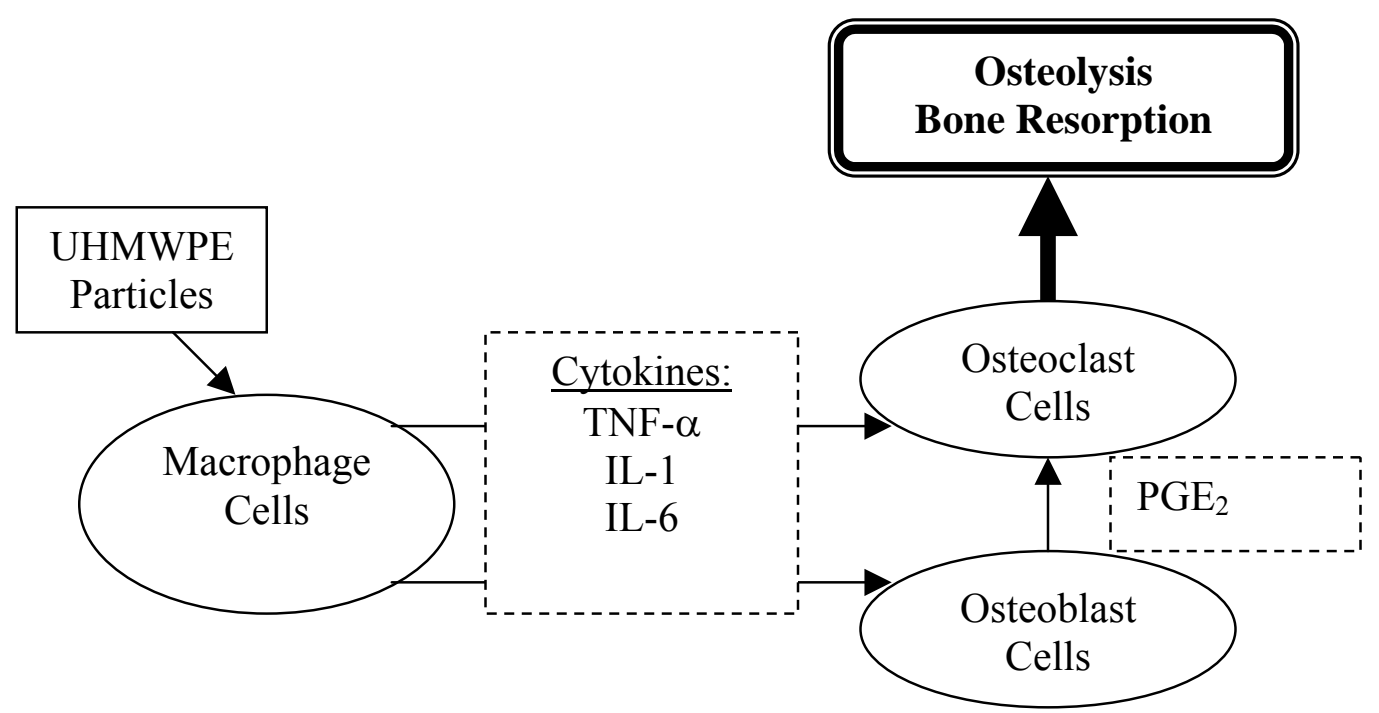

Figure 10.4 Schematic of mechanism for particles-induced osteolysis. 


\subsection{Phagocytosis of particles}

From a biophysical viewpoint of the phagocytosis of particles, the mechanisms of particle-induced bioactivity can be divided into three categories: 1) Phagocytosis of phagocytosable particles plays the most important role in the process. A quantitative calculation of the phagocytosis mechanism will be introduced to determine the dependence of the phagocytic capacity on particle size, shape and dosage factors. 2) In addition, the effects of excess phagocytosable particles that cannot be internalized due to insufficient amounts of macrophage cells should be discussed. 3) The third category involves the non-phagocytosable particles that are too large for macrophage cells to engulf. This leads to some formation of giant cells and is not considered in this study. Cells tend to attach to the particle surface and wrap around the particle. Some studies have compared various sizes and shapes of particles in this category and have shown stimulated inflammatory responses as well. A schematic illustration of the three different categories from the viewpoint of phagocytosis is presented in Figure 10.5.

In the following sections, the effects of internalized particles, effects of excess phagocytosable particles, and effects of non-phagocytosable particles will be discussed. Results of biological response of particles from the literature will be compared. 


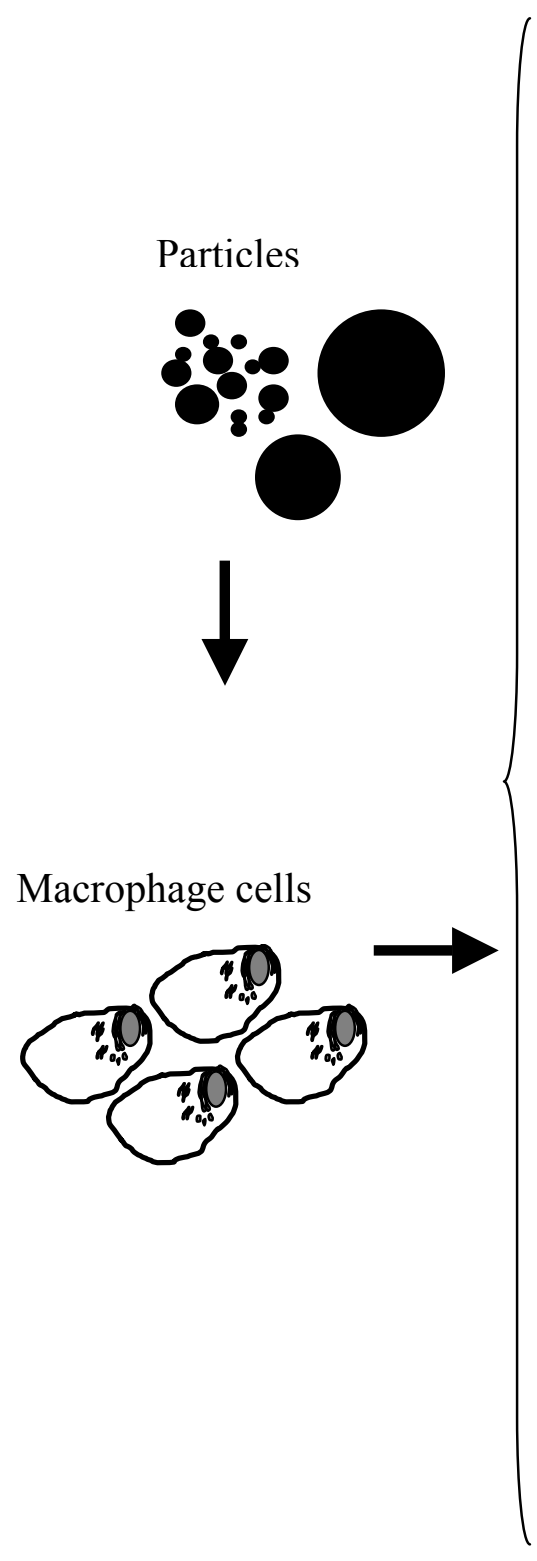

1. Internalized particles:

Macropahge cells engulf the particles inside the cells

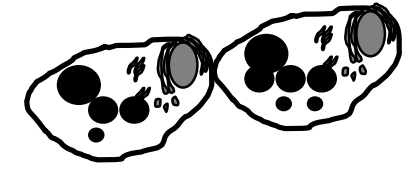

2. Excess phagocytosable particles:

Particles are small enough to be internalized, but insufficient cells are available )

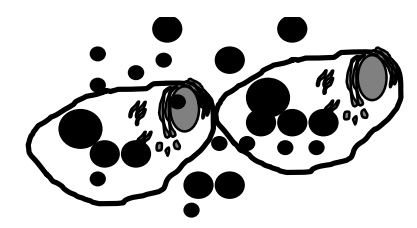

3. Non-phagocytosable particles:

Macrophage cell is too small to internalize large particles, they tend to attach to the particles)

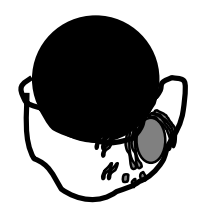

Figure 10.5 Schematic particles-induced bioactivity from viewpoint of phagocytosis. 


\subsubsection{Criteria for particle internalization by macrophage cells}

Macrophage cells have adopted a variety of mechanism to internalize particles including pinocytosis and phagocytosis. Pinosytosis usually refers to the uptake of the smaller particles (usually $<0.5 \mu \mathrm{m}$ ) with fluid during the process. Although macrophage cells cannot digest polyethylene, particles are taken up with extracellular fluid through pinocytosis without stimulating active cell response such as key cytokines (TNF- $\alpha$, IL-1, and IL-6) [86]. For larger particles, macrophage cells are activated according to the following phagocytosis process. Engulfment of a particle involves the following steps: adhesion between cell and particle, cell activation and projection of membrane-bound cytoplasm around the particle, and membrane fusion for enclosure $[114,115]$. There exists a "phagocytic capacity" of a cell that will no longer take up additional particles. The number of particles a cell will engulf appears to depend on several aspects: cell type and morphometry, cell growth environment, chemical composition of the particle, surface property of the particle, and size and shape of the particle. The particle parameters cannot be changed independently to analyze the particle size, shape, dosage (number of particles, surface areas, volumes) effects individually. We proposed the level of activation expressed by macrophage cells depends on the degree of phagocytic capacity of that group of cells. From this concept, all particle parameters can be embedded in the calculation of phagocytic capacity and compared with the level of biological responses. 


\subsubsection{Concept of phagocytic capacity}

The typical macrophage cells are spherical-like in shape and consist of numerous of different organells. They are nucleus, endoplasmic reticulum, centrosome, and centrioles, Golgi apparatus, cytoskeleton, cytosol, mitochondria, lysosomes, and vacuole. Cytoplasmic and intracellular membranes are significant for phagocytosis. The membrane surface areas that are available for phagocytosis have been measured by microscopic techniques. Summation of surface area of intracellular and plasma membranes has demonstrated that in phagocytosing macrophages the total area of these membranes remains constant $[116,117]$. The intracellular membranes include the compartment of vacuoles that are empty spaces inside the cell and the compartment of phagosomes in which the particle is internalized. It is recognized that for a macropahge cell with initial volume $V_{0}$, the total cytoplasmic membrane area $S_{0}$ is available for phagocytosis. The assumption is that during the internalization process, the membrane of the phagocytic vacuole is closely attached to the particle surface and each particle is internalized by a different vacuole. For a particle with volume $V_{\mathrm{p}}$, and surface area $S_{\mathrm{p}}$, the maximum number of particles can be phagocytosed is denoted as $n$. After the phagocytosis process, the remaining cytoplasmic membrane area is $S$, and the new cell volume is $V$ :

$$
\begin{aligned}
& V=V_{0}+n V_{\mathrm{p}} \\
& S=S_{0}-n S_{\mathrm{p}}
\end{aligned}
$$

Assuming that in the limit of complete plasma membrane engulfment the cell is a sphere with radius $r$, so that $V=(4 / 3) \pi r^{3}$ and $S=4 \pi r^{2}, n$ has been found as the thirdorder polynomial [118]: 


$$
n^{3} a_{3}+n^{2} a_{2}+n a_{1}+a_{0}=0
$$

with coefficients

$$
\begin{gathered}
a_{0}=36 \pi V_{0}^{2}-S_{0}^{3}, \\
a_{1}=72 \pi V_{0} V_{\mathrm{p}}+3 S_{0}{ }^{2} S_{\mathrm{p}}, \\
a_{2}=36 \pi V_{\mathrm{p}}{ }^{2}-3 S_{0} S_{\mathrm{p}}{ }^{2}, \\
a_{3}=S_{\mathrm{p}}{ }^{3} .
\end{gathered}
$$

A measurement of the total membrane surface area showed that the membranes have $300 \%$ to $350 \%$ of the surface area of a sphere with a volume $V_{0}$. It represents a maximum amount of membrane area that could be used for phagocytosis of particles. During the phagocytosis process, vacuolar membranes tend to shift for phagocytosis. However, several studies have indicated that around $35 \%$ to $55 \%$ of membrane areas remained in the vacuoles compartments that cannot be used for phagocytosis purpose. Bos and Souza [116] have reported a measurement of volume and surface area of macrophage cells and its vacuoles and phagosomes compartments. For a macrophage cell before phagocytosis process, the volume was $388 \mu \mathrm{m}^{3}$. The total membrane surface area measured was $1231 \mu^{2}$. Assuming the maximum percentage of total membrane surface area used for phagocytosis as $65 \%$, we can obtain that the maximum diameter of the sphere that can be engulfed is $9 \mu \mathrm{m}$. The membrane area that is available for phagocytosis $S_{0}=800 \mu \mathrm{m}^{2}$. We also estimate that if the minimum percentage of total membrane surface area used for phagocytosis is $45 \%$, the available surface area available for phagocytosis is $S_{0}=554 \mu \mathrm{m}^{2}$. 


\subsubsection{Critical threshold for phagocytosis of particles with different shapes}

For a population of spherical particles with the same size, the range of phagocytic capacity as the number of internalized particles can be determined. The largest spherical particle that can be internalized has a diameter of (8.5 to 10$) \mu \mathrm{m}$. We also calculated the phagocytic capacity for different sizes of spherical particles. The phagocytic capacity versus diameter of spherical particles is plotted in Figure 10.6.

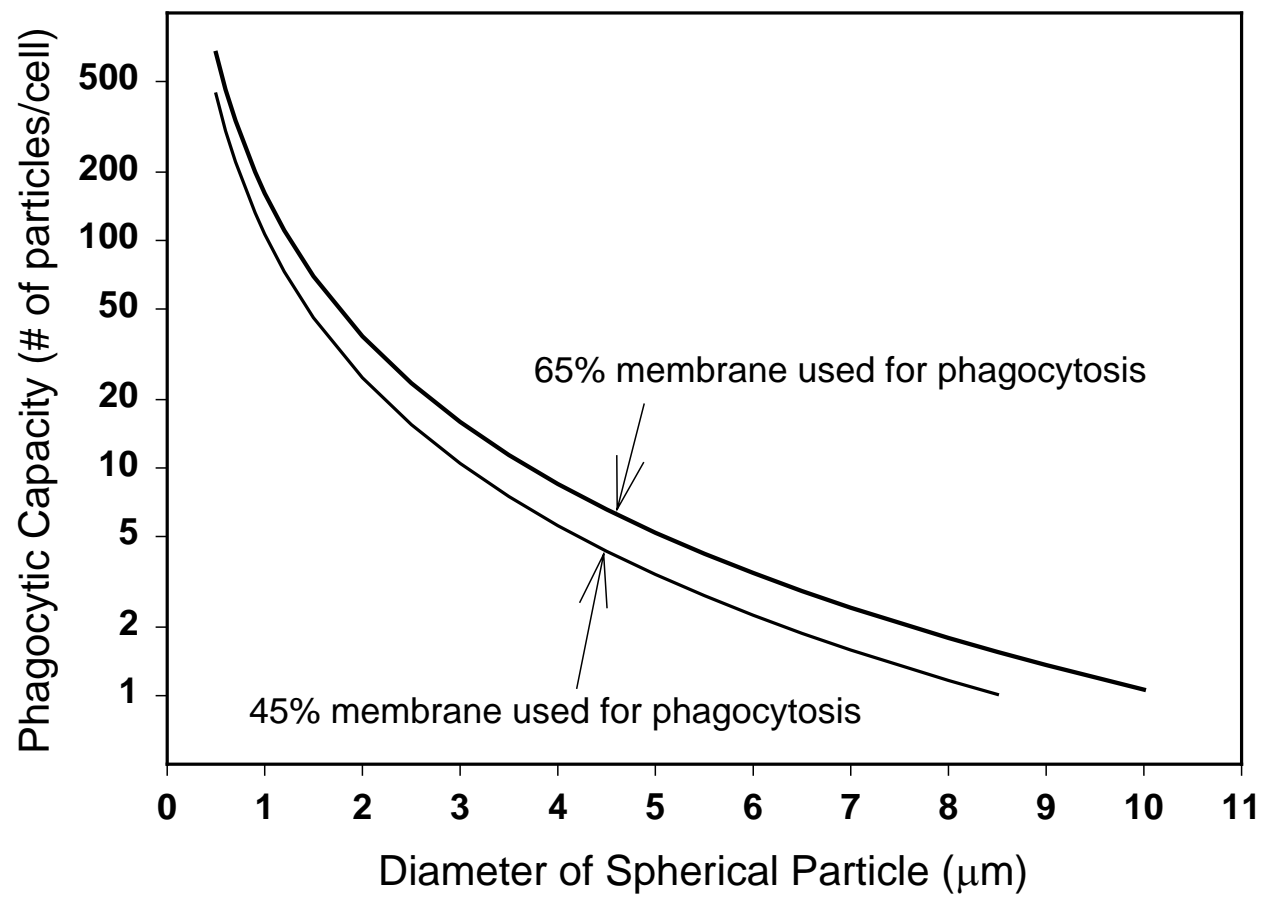

Figure 10.6 Phagocytic capacity of spherical particles. 
To discuss the phagocytic capacity of elongated particles, we assume that the particle has an ellipsoidal shape with longer axis $L$ and two equal shorter axes $W$. The aspect ratio of the particle $(A R)$ is defined as $L / W$. The projected surface area can be calculated from equation 10.4. By using the same set of equations, we can determine the phagocytic capacity for elongated particles. Based on the assumption that $45 \%$ membrane area is used for phagocytosis, phagocytic capacity versus particle volume for particles with different aspect ratios are plotted in Figure 10.7. The figure indicates that for the same volume of particle, the phagocytic capacity decreases with increasing aspect ratio of the particle. Obviously, with the same particle volume, a sphere has the minimum surface area and the largest phagocytic capacity.

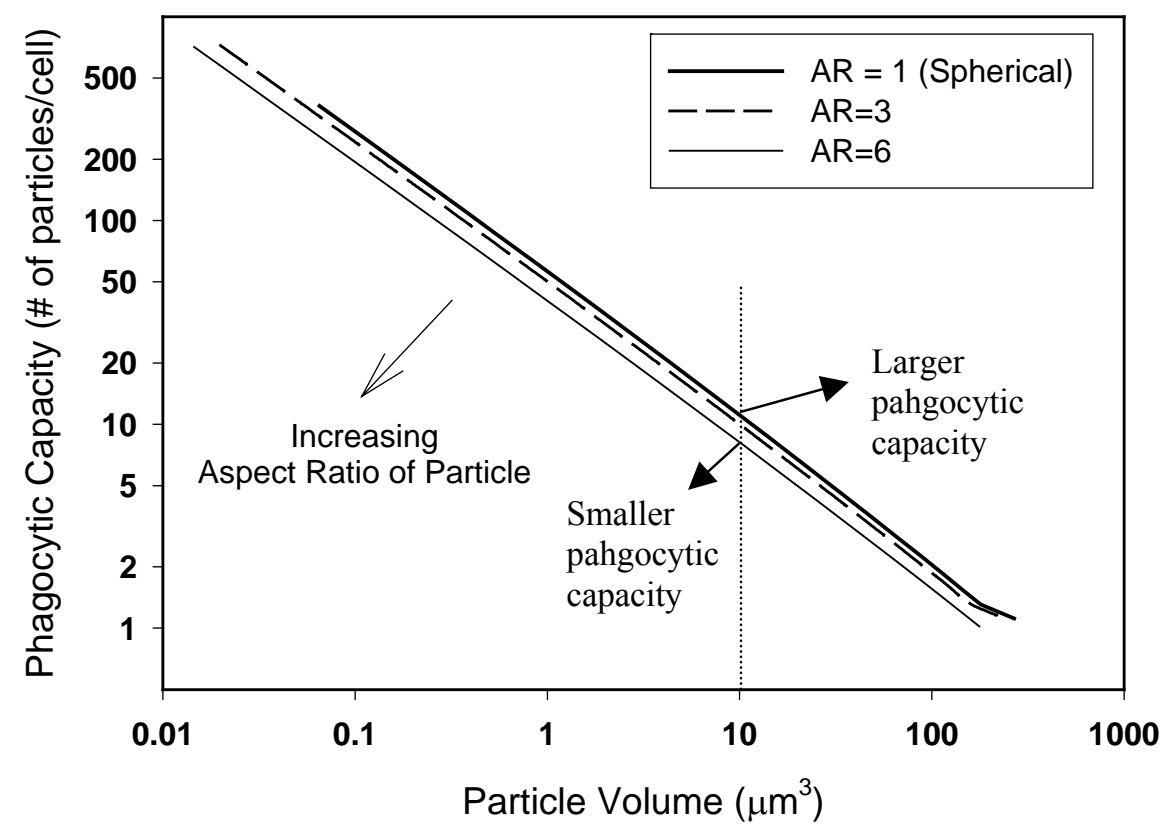

Figure 10.7 Phagocytic capacity of particles with different aspect ratios. 


\subsection{Validation against literature results}

To investigate the relationship between phagocytic capacity and related biological response, two cell-culture studies of polyethylene particles are reviewed and analyzed. Because no elongated particles have been used for biological response tests, all the particles mentioned below are spherical shaped.

Catelas et al. [79] studied different kinds of particles in the phagocytosable range, including HDPE powders with a mean diameter of $4.5 \mu \mathrm{m}$. The J774 macrophage cell line (ATCC) was used in this vitro model because it has been demonstrated to be morphologically similar to macrophages at the bone-cement interface and to respond to particles in a similar way as human macrophages at this interface. Macrophages were cultured in a tube that was placed on a rotator during the incubation to facilitate constant contact between cells and particles. Dosage baseline used in this study is the ratio of number of particles to the number of macrophage cells. The TNF- $\alpha$ released after $24 \mathrm{~h}$ was measured and the results for HDPE particles is shown in Table 10.1. The phagocytic capacity of a spherical particle with $4.5 \mu \mathrm{m}$ in diameter ranges from 3 (45\% membrane for phagocytosis) to 6 (65\% membrane for phagocytosis) based on the calculation algorithm mentioned earlier. The ratio of the particle concentration (no. of particles/no. of cells) to the phagocytic capacity was calculated. This ratio is less than unity when there are sufficient cells to phagocytose particles. This ratio indicates the level of cell phagocytic capacity is used. This ratio is larger than unity when the cells are not able to internalize all the particles and there 
exist excess phagocytosable particles in the system. The TNF- $\alpha$ specific activity versus index of particle internalization is plotted in Figure 10.8

Table 10.1 Summary of in-vitro cell culture results from Catelas et al. [79]

\begin{tabular}{|c|c|c|c|}
\hline \multirow{2}{*}{ HDPE particles $4.5 \mu \mathrm{m}$} & \multicolumn{3}{|c|}{ Particle concentration (/cell) } \\
\cline { 2 - 4 } & 5 & 30 & 80 \\
\hline TNF- $\alpha$ specific activity & $1.6 \pm 0.2$ & $2.6 \pm 0.18$ & $2.75 \pm 0.5$ \\
\hline
\end{tabular}

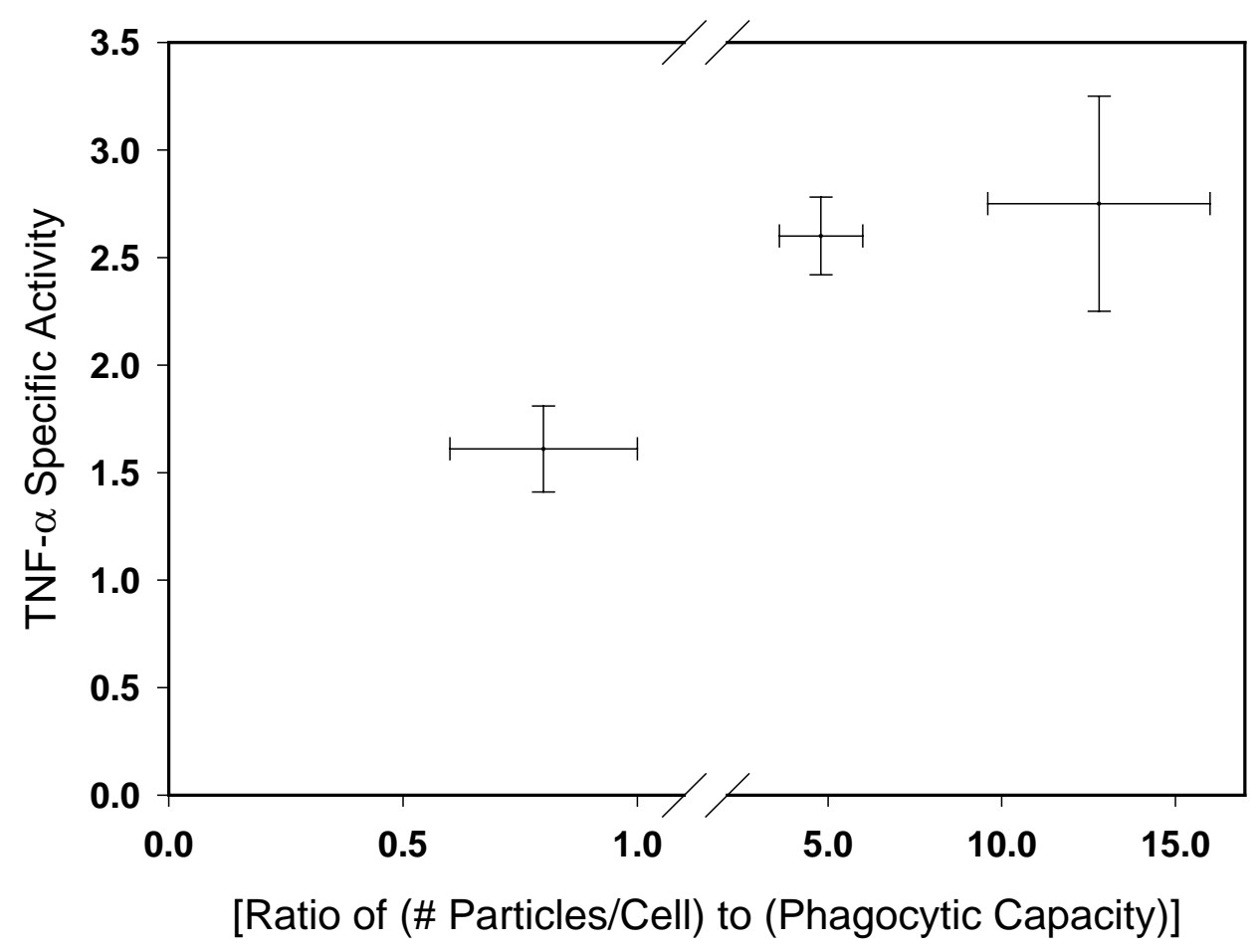

Figure 10.8 TNF- $\alpha$ vs. index of particle internalization from Catelas et al. [79]. 
It is seen from Figure 10.8 that the TNF- $\alpha$ specific activity is smaller when the ratio of the particle concentration (no. of particles/no. of cells) to the phagocytic capacity is smaller than unity. It means the cells have not activated fully to the stimulated particles. When the number of particles is increased, the TNF- $\alpha$ specific activity increases as well. However, after the cells reach their phagocytic capacity, the TNF- $\alpha$ specific activity remained constant in this study. It indicates that the ability of cells to release TNF- $\alpha$ is fully activated when saturation of phagocytic capacity is reached and the excess particles in this case seem not to have much impact on the TNF- $\alpha$ release. The size effect of the particles was not investigated in this study.

Green et al. [81] took Ceridust 3615 polyethylene powders and categorized them into the different size ranges by filtration. They obtained spherical-like particles with mean particle diameters \pm standard deviations of $(7.2 \pm 3.15) \mu \mathrm{m},(4.3 \pm 1.89) \mu \mathrm{m}$, $(0.49 \pm 0.11) \mu \mathrm{m}$, and $(0.21 \pm 0.069) \mu \mathrm{m}$. The macrophage cells were isolated from $\mathrm{C} 3 \mathrm{H} / \mathrm{Hej}$ mice. They fixed polyethylene particles in an agarose solution, following centrifugation to put particles in the superficial layer. The macrophage cells then cultured above the layer in order to make the contact between particles and cells. They controlled the particle concentration in a form of particle volume $\left(\mu \mathrm{m}^{3}\right)$ to macropahge cell number ratios of 100:1, 10:1, 1:1, and $0.1: 1$. Several cytokines were measured after the experiment. They found that the particle concentrations of $1: 1$ and $0.1: 1 \mathrm{did}$ not stimulate cells to release cytokines. Table 10.2 lists the results of IL-1 $\beta$ and TNF$\alpha$ release with different particle concentrations. 
We further converted the particle concentration to the ratio of number of particles to number of macrophage cells. The ratio of the particle concentration (no. of particles/no. of cells) to the phagocytic capacity is again calculated for the particles with different size and concentrations. The IL- $1 \beta$ and TNF- $\alpha$ specific activities versus the ratio of the particle concentration (no. of particles/no. of cells) to the phagocytic capacity are plotted in Figures 10.9 and 10.10, respectively. It is found that the cytokine specific activity increases with increasing phagocytic capacity. When the phagocytic capacity is larger than unity, cytokine specific activity reaches a saturated value and no longer increases within the experimental conditions. When the ratio is between 0 and 1, the bioactivity (cytokine release) increases with increase ratio of the particle concentration (no. of particles/no. of cells) to the phagocytic capacity. Although the particle concentration is rather high (high index of particle internalization), the cytokine release remains low compared to the control experiment. It is thought that this may be that the particles are so tiny so that pinocytosis takes place to uptake the smaller particles with fluid during the process, and therefore, a severe biological response may not happen under this condition.

Table 10.2 Summary of in-vitro cell culture results from Green et al. [81]

\begin{tabular}{|c|c|c|c|c|}
\hline \multirow{3}{*}{ Particle size } & \multicolumn{2}{|c|}{ IL-1 $\beta$ specific activity } & \multicolumn{2}{c|}{ TNF- $\alpha$ specific activity } \\
\cline { 2 - 5 } & \multicolumn{2}{|c|}{$\begin{array}{c}\text { Particle concentration } \\
\text { (particle volume: no. of cells) }\end{array}$} & $\begin{array}{c}\text { Particle concentration } \\
\text { (particle volume: no. of cells) }\end{array}$ \\
\cline { 2 - 5 } & $10: 1$ & $100: 1$ & $10: 1$ & $100: 1$ \\
\hline $0.21 \mu \mathrm{m}$ & $0.2 \pm 0.15$ & $0.3 \pm 0.1$ & $2 \pm 3$ & $8 \pm 8.2$ \\
\hline $0.49 \mu \mathrm{m}$ & $0.6 \pm 0.1$ & $1.8 \pm 0.2$ & $38 \pm 1$ & $33 \pm 5$ \\
\hline $4.3 \mu \mathrm{m}$ & $0.5 \pm 0.15$ & $1.75 \pm 0.25$ & $15 \pm 10$ & $35 \pm 2$ \\
\hline $7.2 \mu \mathrm{m}$ & $0.1 \pm 0.09$ & $1.1 \pm 0.3$ & $1 \pm 0.8$ & $16 \pm 10$ \\
\hline
\end{tabular}




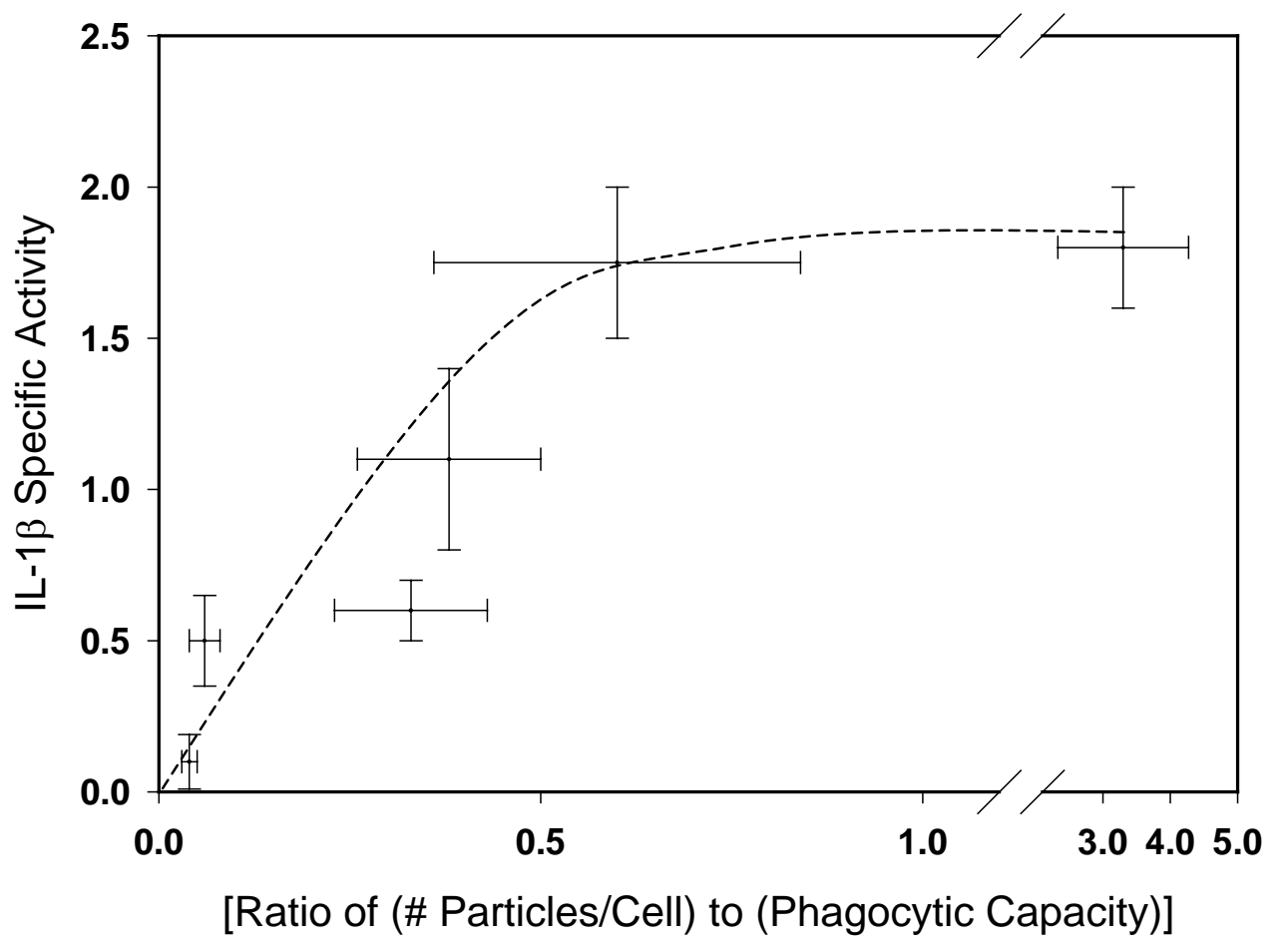

Figure 10.9 IL-1 $\beta$ vs. index of particle internalization from Green et al. [81]. 


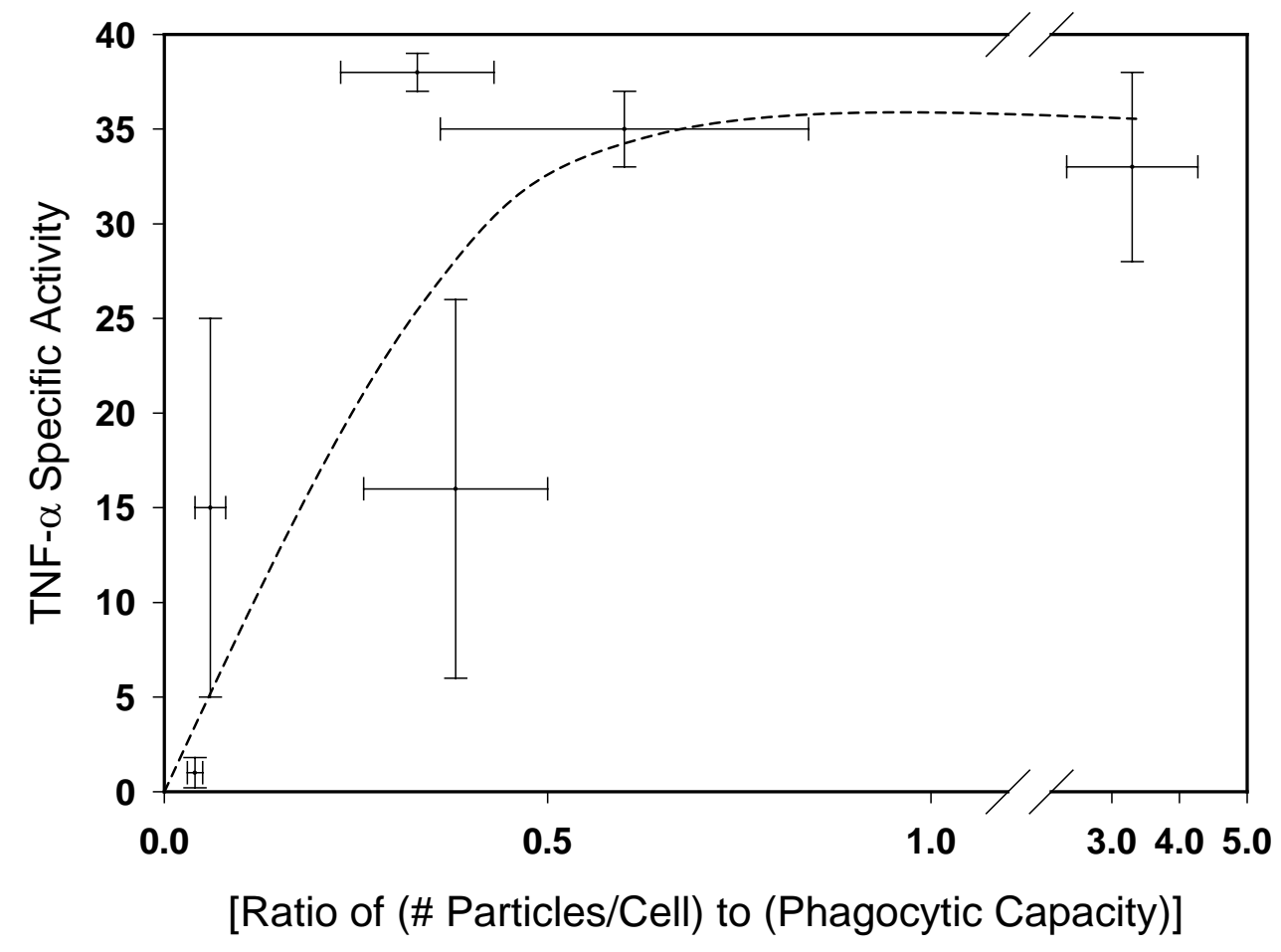

Figure 10.10 TNF- $\alpha$ vs. Index of particle internalization from Green et al. [81]. 


\subsection{Effect of excess particles}

The case studies indicated that when ratio of the particle concentration to the phagocytic capacity is larger than unity, the bioactivity reaches a saturation state without further increase. It means the cells are fully activated and there is no capacity left for further phagocytosis of particles. The two studies that are referenced $[79,81]$ were conducted in cell-culture studies with the condition of the cell cultures fixed after the experiment has begun. Compared to the dynamic particles-cells interaction in the joint area, it is a static process. However, in the joint area, the dynamic interaction includes the generation rate of the particles and the growth period of related immunological cells. It has been observed in animal studies that after the macrophage cells internalize the particles, more macrophage cells appear from the surrounding tissues for further phagocytosis. Thus whether the phagocytic capacity is saturated or not is no longer only a issue related to particle size, number and cell numbers, but also a topic involving the wear-particle generation rate and the growth periods of macrophage cells. Figure 10.11 shows a schematic of the dynamic process of this concept. Further efforts should be made to control the cell and particle-generation rate to study these dynamic effects. 


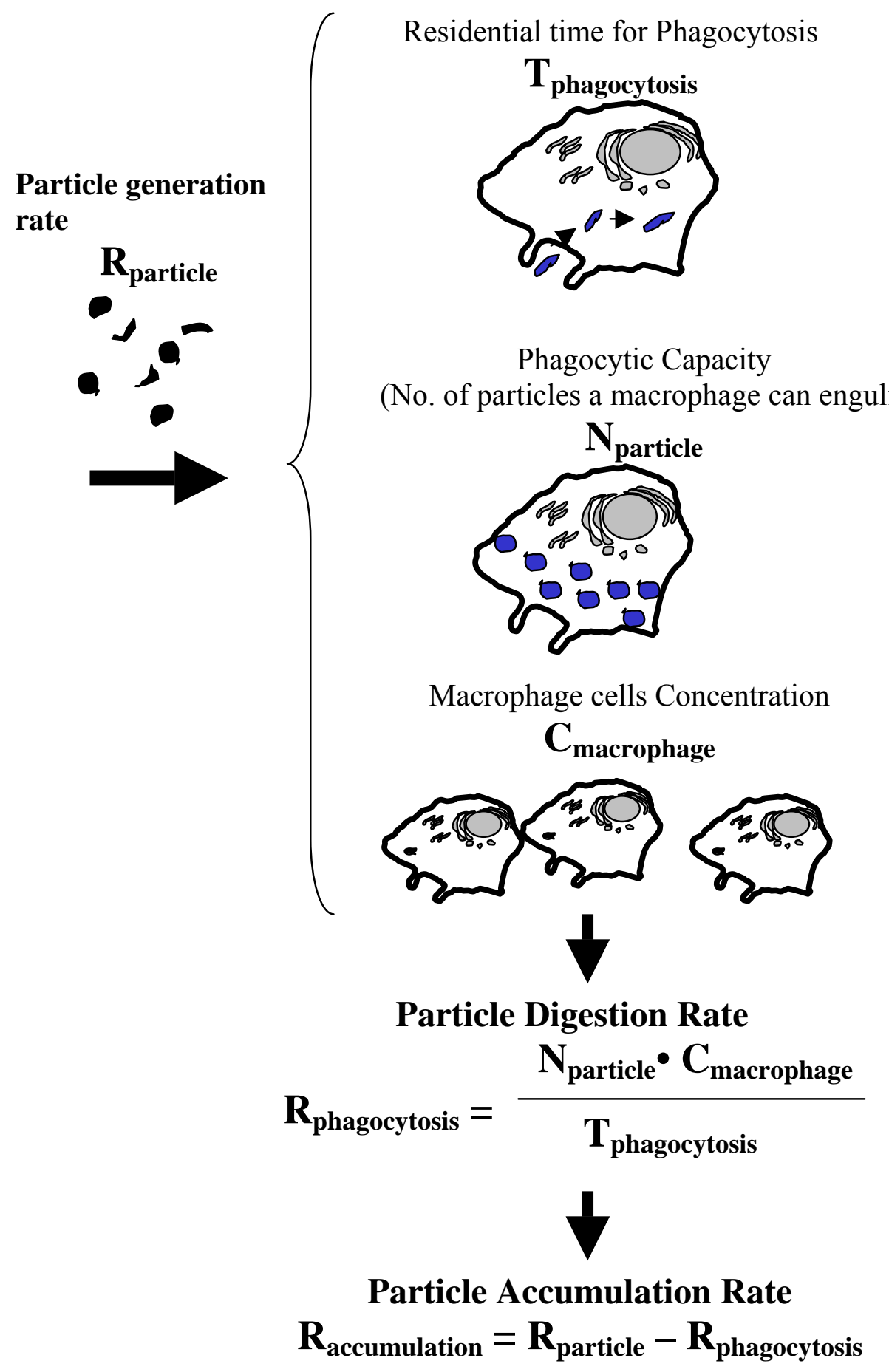

Figure 10.11 Schematic of dynamic phagocytosis model. 


\subsection{Conclusions}

Methods to describe the size and shape of particles are reviewed first in this chapter. However, according to the positive and negative curvature of the sheet-like UHMWPE particles generated, there exists difficulty to achieve precisely measurement of the dimensions and aspect ratios of the particles. Accordingly, a projected area concept was proposed that reflects the size of the particle based on the physical aspect of phagocytosis of particle mechanism. We have analyzed the phagocytosis of particles from a biophysical viewpoint. The phagocytic capacity of a particle with a certain size and shape can be determined. The ratio of the particle concentration (no. of particles/no. of cells) to the phagocytic capacity is a critical value for the bioactivity induced by a certain particle population. This ratio integrates the effects from particle size, shape and dosage. The literature results of bioactivity tests of spherical particles have been reviewed. When the ratio is smaller than unity, bioactivity is proportional to the value of the ratio. When the ratio is larger than unity, limited change of biological response was observed.

Elongated UHMWPE particles that are seen clinically have not been used in bioactivity tests due to the unavailability of the elongated particles. In the previously chapters, we have successfully generated elongated particles with different aspect ratios. We shall apply these elongated particles in the bioactivity test to investigate the shape effect. We also can further test the mechanism proposed in this chapter. 


\section{CHAPTER 11}

\section{BIOACTIVITy TeSTS OF UHMWPE PARTICLES}

\subsection{Introduction}

In this chapter, the results of collaborative research with Wayne State University on bioactivity tests of UHMPWE particles is presented ${ }^{1}$. They investigated the effects of particle size and shape on biological response, and we analyzed the combination of particle size, shape and dosage effects on bioactivity.

In-vitro cell culture tests and in-vivo animal tests have been widely used to evaluate biomaterials induced immunological responses [78-87]. However, in-vitro cell-culture studies may not be able to mimic the in-vivo environment because of the lack of human biochemical complexity. Some researchers applied in-vivo animal studies by injecting the wear particles into the joint areas. However, quantitative analysis became impossible because of lack of control of the experimental parameters. The Rodent air pouch has been identified as a useful model for the evaluation of the response to orthopedic biomaterials, providing inflammation responses resembling the pseudo-synovium associated with aseptic loosening [122]. We adopted the murine air pouch model here to evaluate the cellular response to UHMWPE particles in vivo.

${ }^{1}$ Acknowledge Professor Paul Wooley, Dr. Shang-You Yang and Dr. Weiping Ren at Wayne State University for conducting murine air pouch animal tests. 


\subsection{Murine air pouch animal test}

Air pouches were generated in groups of 10-15 female BALA/c mice. An area of the dorsal skin $\left(2 \mathrm{~cm}^{2}\right)$ was cleaned with alcohol and shaved to provide the pouch site. Three milliliters of air was injected at a single site with 25 -gauge needle and $3 \mathrm{ml}$ syringe. The air pouches were injected with $1 \mathrm{ml}$ of air on alternate days for 5 days to establish a definitive fluid filled pouch [123]. On day 6, the established air pouches were injected with $500 \mu \mathrm{L}$ sterilized phosphate buffer solution (PBS) containing $10 \%$ fetal calf serum (FCS) and controlled amounts of UHMWPE particles. Particle-less control pouches were injected with $500 \mu \mathrm{L}$ of $10 \%$ FCS/PBS alone. The mice were sacrificed at defined intervals subsequent to particle introduction. The pouches were lavaged with $500 \mu \mathrm{L}$ of PBS (collected as lavage fluid and rapidly frozen at $-80^{\circ} \mathrm{C}$ for later evaluation) before the surgical removal of the pouch. One half of each pouch was fixed in $10 \%$ buffered formalin for histology processes, while the remainder was stored at $-80^{\circ} \mathrm{C}$ for molecular and immunological analyses. Figure 11.1 shows a schematic of the processes of the murine air pouch tests for particles. 

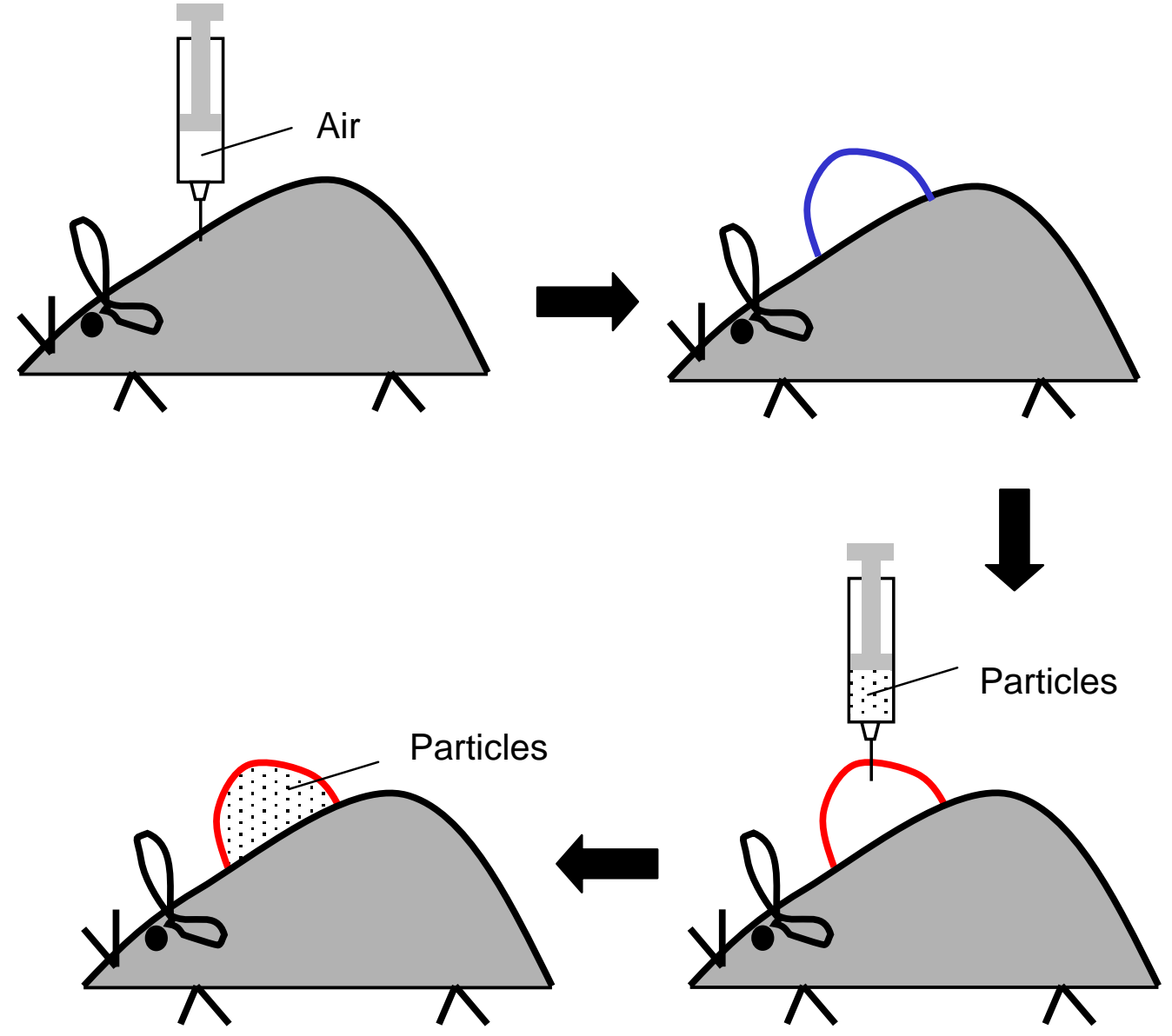

Figure 11.1 Schematic of murine air-pouch model for bioactivity tests of UHMWPE particles. 


\subsection{Evaluation of biological responses}

\subsubsection{Histology analysis ${ }^{2}$}

Formalin-fixed pouches were embedded in paraffin, sectioned at $5 \mu \mathrm{m}$ with a consistent orientation. The sections were analyzed under a Zeiss light microscope by a computerized image analysis system with the software. For pouch membrane thickness, eight measurements from different areas of each pouch were averaged. A uniform $100 \mu \mathrm{m}$ longitudinal pouch area was selected to count total cells (H\&E stained) and macrophages (Esterase positive stained) [123], respectively, and average of three different areas per pouch was recorded.

Figure 11.2 shows a typical micrograph of the tissue reaction in an air-pouch membrane injected with UHMWPE particles, with particle phagocytosis by macrophage cells. Figure 11.3 illustrates typical macroscopic appearances of air pouches dissected from mice. Figure 11.4 shows the different level of immunological responses by quantification of the pouch thickness.

${ }^{2}$ Histology analyses of the murine air-pouch membranes were done by the research staffs in the department of orthopedics at Wayne state university. 

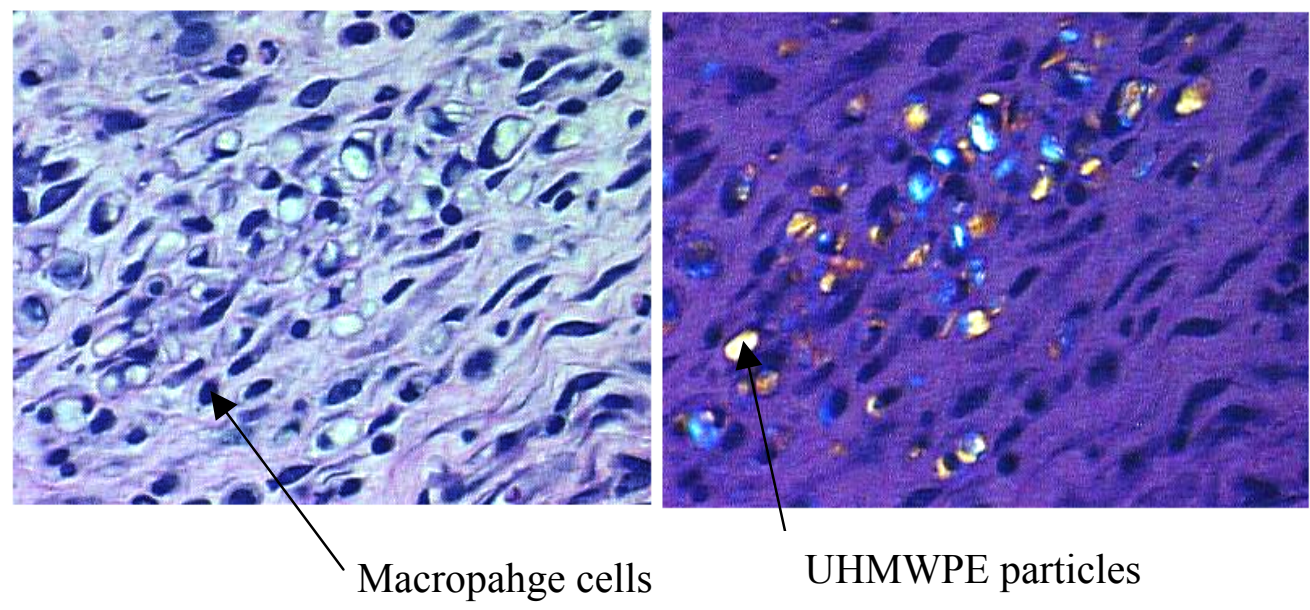

Figure 11.2 Micrographs of phagocytosis of UHMWPE particles by macrophage cells.

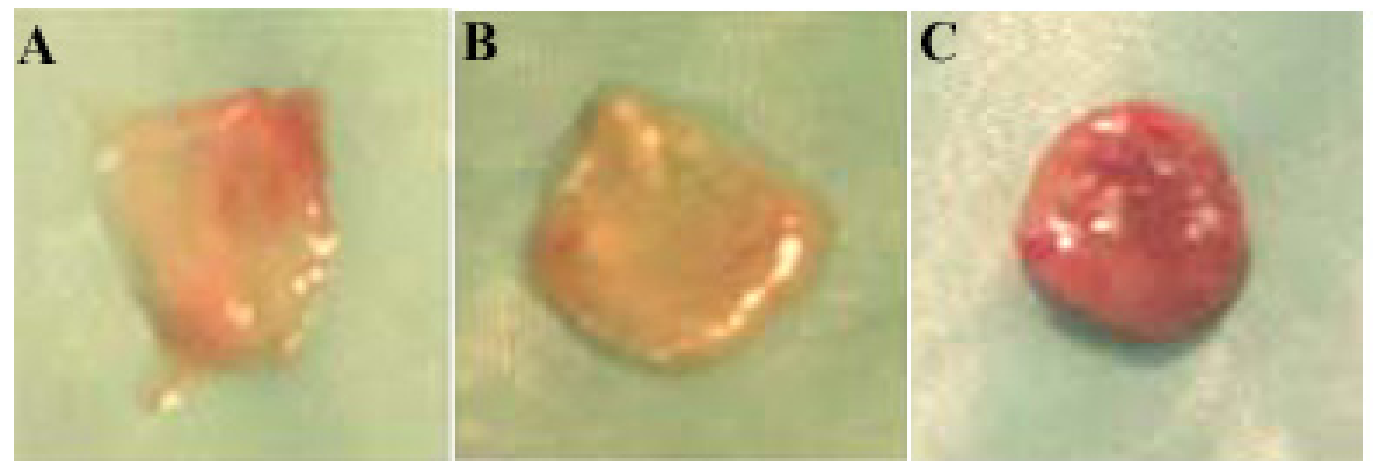

Figure 11.3 Photographs of murine air pouches; (a) without particle; (b) reacted with round-like particles; (c) reacted with elongated particles. 

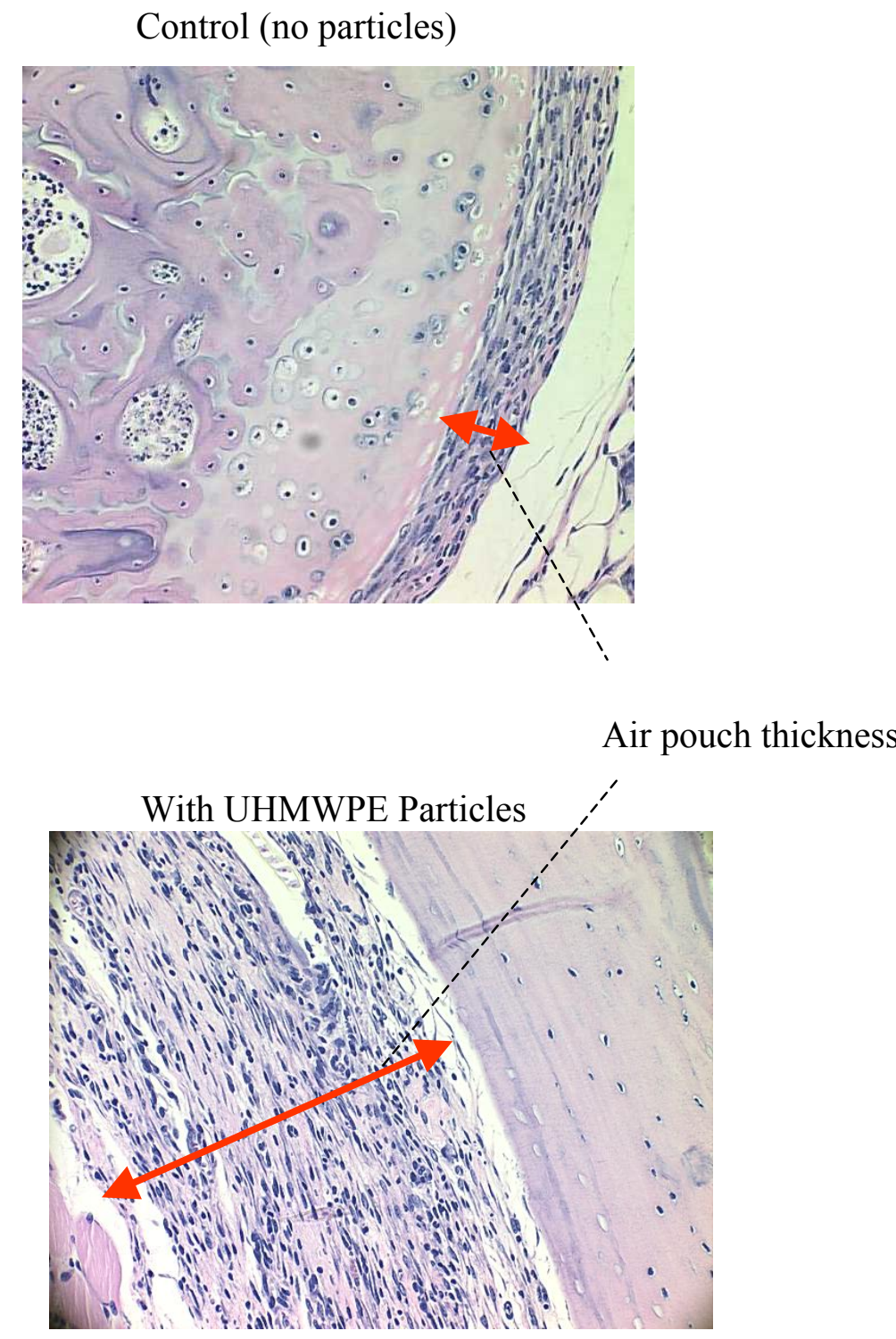

Figure 11.4 Micrographs of sectioned air pouches. 


\subsubsection{Measurement of cytokines ${ }^{3}$}

Enzyme Linked Immunosorbent Assay (ELISA) was performed to examine cytokine production at the protein level using capture and detection monoclonal antibody pairs against various cytokine molecules. $50 \mu \mathrm{L}$ of sample (pouch lavage fluid, supernatant of pouch homogenates, or cell culture media) was added to each well of a 96-well plate pre-coated with capturing antibody. The plate was incubated overnight at $4{ }^{\circ} \mathrm{C}$ and biotinylated detection antibody was added for 90 min at $37{ }^{\circ} \mathrm{C}$. Alkaline phosphatase-conjugated streptavidin (1:500) was introduced to each well and incubated for $45 \mathrm{~min}$ at room temperature. Extensive washes with PBS were applied following each incubation step. The optical density of each sample was measured by a microplate spectrophotometer (Molecular Devices, Menlo Park, CA) at $405 \mathrm{~nm}$ wavelength, after incubating the plate with paranitrophenyl phosphate in diethylanolamine buffer for $15 \mathrm{~min}$ to $30 \mathrm{~min}$ in dark. The level of cytokines was calculated by regression analysis from a standard curve included with each assay.

The expression of inflammatory cytokines in the air pouches was also quantified by a real-time PCR technique. Detailed procedures have been described in the literature [124].

${ }^{3}$ Cytokine was measured by Dr. Shang-You Yang at Wayne state University. 


\subsection{Effect of particle shape}

UHMWPE particle populations with different aspect ratios of particles were tested in the murine air pouch animal tests. Particle sizes were chosen so that the average surface areas of the particles are similar for two particle populations. Particle group (A) contained round-like particles with mean particle length $(22.8 \pm 3.0) \mu \mathrm{m}$, particle width $(17.5 \pm 2.5) \mu \mathrm{m}$, and aspect ratio of (1.3 \pm 0.2$)$. Particle group (B) contained much elongated particles with mean particle length $(26.9 \pm 1.1) \mu \mathrm{m}$, particle

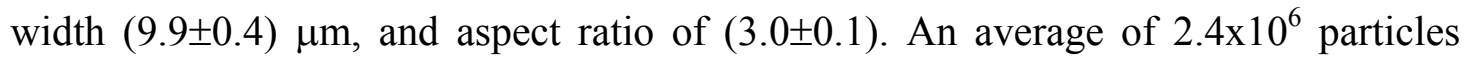
$\left(4.8 \times 10^{6}\right.$ particles $\left./ \mathrm{ml}\right)$ were contained in the suspension for injection to the air pouch for both particle groups.

Both populations of particles induced tissue inflammatory responses, including increases cellular infiltration and membrane proliferation. Differences were observed between the responses to the different particle populations, with elongated particles provoking more severe inflammatory responses. Figure 11.3 illustrates typical macroscopic appearances of air pouches dissected from mice. Pouches with elongated particles (Figure 11.3c) had pronounced more changes compared with other two groups.

Image analysis of histological sections revealed that the pouch membranes stimulated with elongated particles (Group B) for 7 days averaged $(0.223 \pm 0.021) \mathrm{mm}$ in thickness, significantly thicker than pouches with round-like particles (Group A) $((0.144 \pm 0.005) \mathrm{mm})$ and non-particle control pouches $((0.071 \pm 0.01) \mathrm{mm})$ as shown in Figure 11.5. 


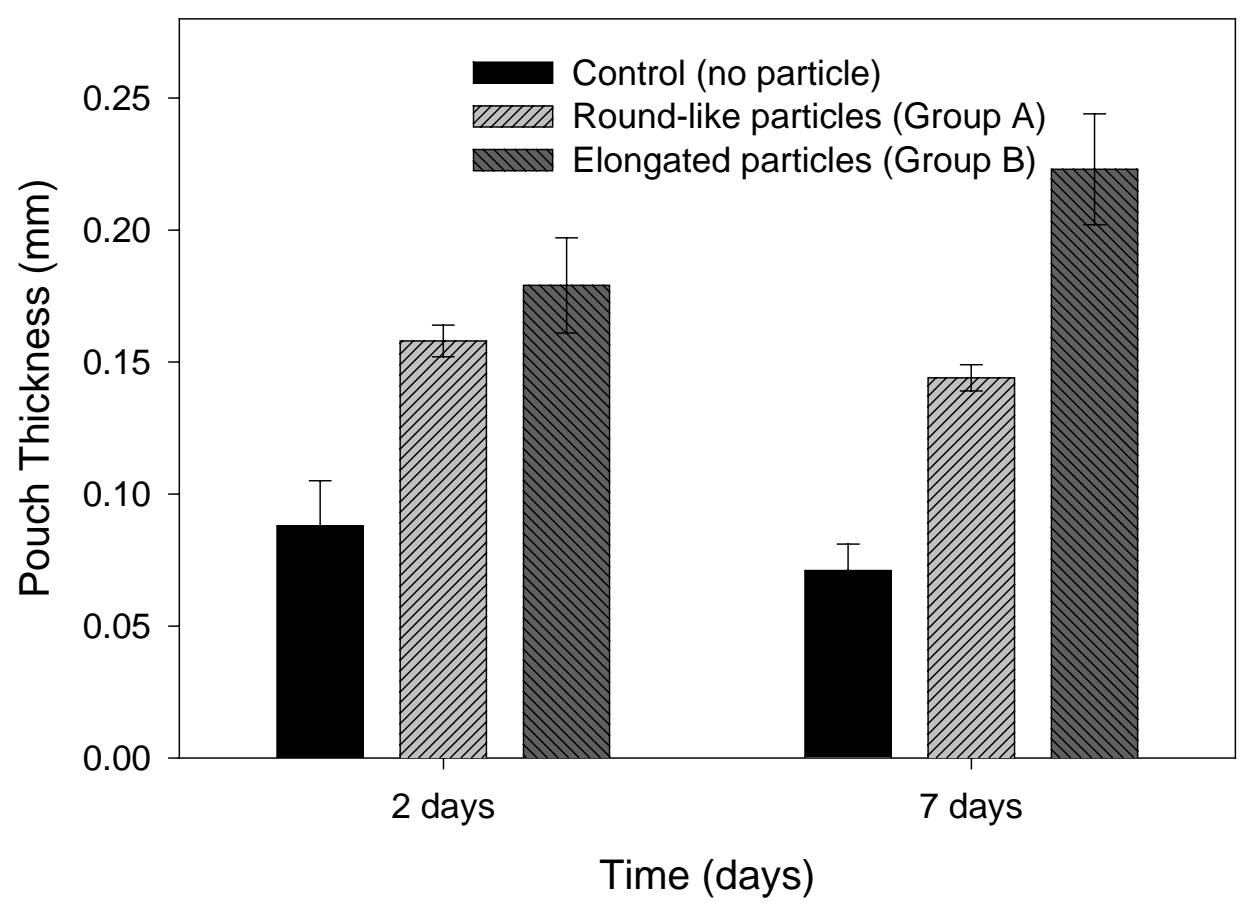

Figure 11.5 Comparison of pouch thickness for the murine air pouch tests with round-like (Group A) and elongated (Group B) UHMWPE particles. 
Cytokine measurements by PCR technique revealed that the UHMWPE particles significantly provoked expression of proinflammatory cytokines IL-1 and TNF at day 2 and day 7 in the air pouch membranes compared to non-particle controls as shown in Figure 11.6. However, mRNA from pouches with elongated-particles contained markedly more gene copies of IL-1 and TNF shown in Figure 11.6a-b, indicating the elongated-particles' higher inflammatory-inducing nature. ELISA assay further confirmed that higher level of IL- $1 \beta$ and TNF $\alpha$ were present in pouch fluid lavaged from elongated-particle treated air pouches in Figure 11.6c-d.

In another experiment, two particle populations with smaller particle size were applied to compare the particles with different aspect ratios. Particle group (C) contains spherical particles with mean particle diameter $(3.6 \pm 2.0) \mu \mathrm{m}$, and aspect ratio of (1.0 \pm 0.3$)$. Particle group (D) contains much elongated particles with mean particle length $(4.2 \pm 0.8) \mu \mathrm{m}$, particle width $(2.6 \pm 0.5) \mu \mathrm{m}$, and aspect ratio of (1.6 \pm 0.3$)$. An average of $0.7 \times 10^{6}$ particles $\left(1.4 \times 10^{6}\right.$ particles $\left./ \mathrm{ml}\right)$ were contained in the suspension for injection to the air pouch for both particle groups. Image analysis of histological sections revealed that the pouch membranes stimulated with elongated-particles (Group D) for $7 \mathrm{~d}$ averaged $(0.215 \pm 0.012) \mathrm{mm}$ in thickness, significantly thicker than pouches with spherical particles (Group C) $((0.138 \pm 0.020) \mathrm{mm})$, and non-particle control pouches $((0.102 \pm 0.01) \mathrm{mm})$.

Both results indicate that the stronger immunological responses were stimulated by the elongated particles than by the round-like or spherical particles while the total surface areas of particles are maintained similar. 

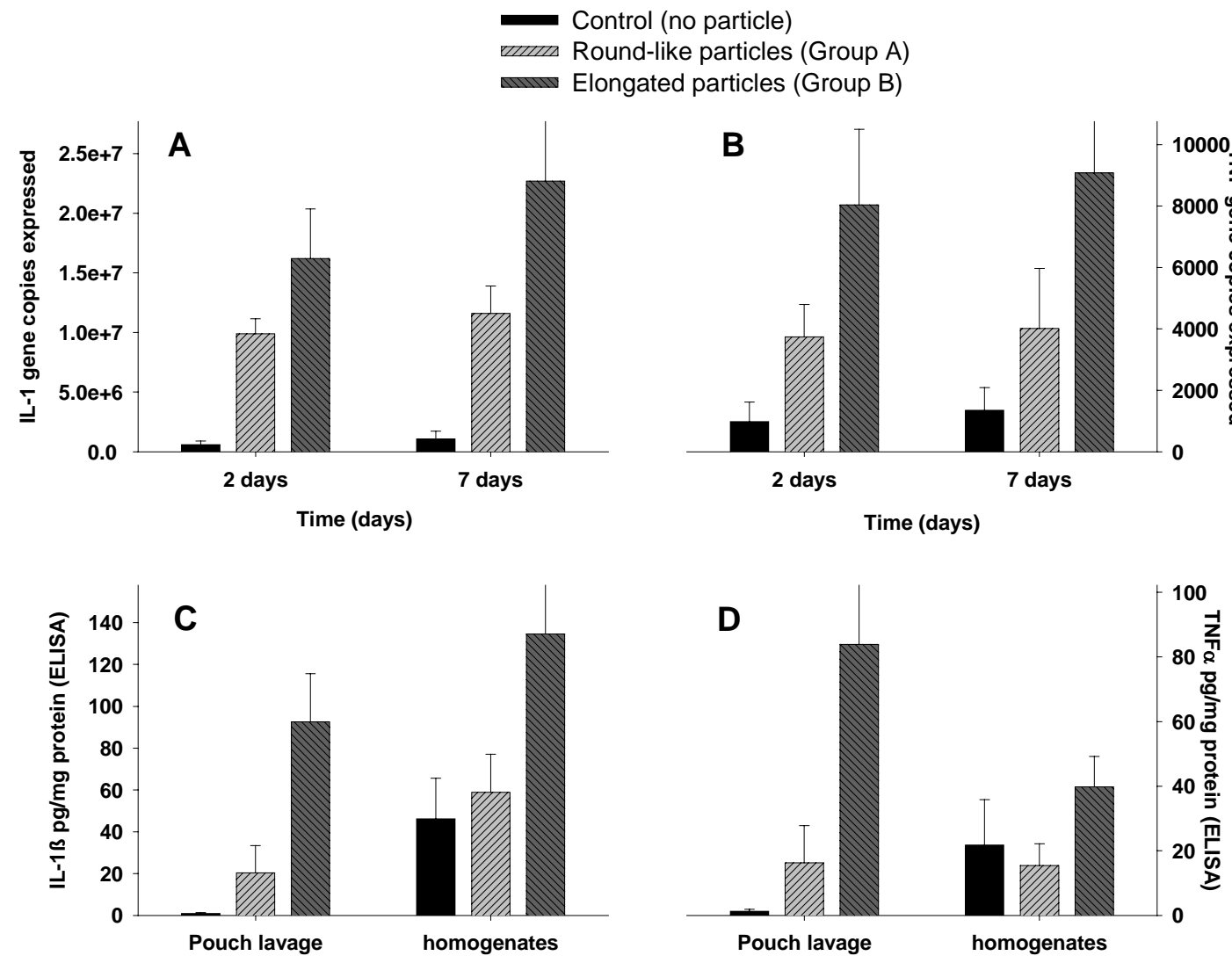

Figure 11.6 IL-1 $\beta$ and TNF- $\alpha$ cytokine expression in murine air pouches with UHMWPE particles by real time PCR (plots A and B) and by ELISA (plots $\mathrm{C}$ and $\mathrm{D}$ ) techniques. 


\subsection{Effect of particle size}

Two populations of elongated UHMWPE particles (Aspect ratio > 1.5) were compared in the air pouch animal tests. Particle group (D) contained smaller particles with mean particle length $(4.2 \pm 0.8) \mu \mathrm{m}$, particle width $(2.6 \pm 0.5) \mu \mathrm{m}$, and aspect ratio of (1.6 \pm 0.3$)$. Particle group (E) contained larger particles with mean particle length

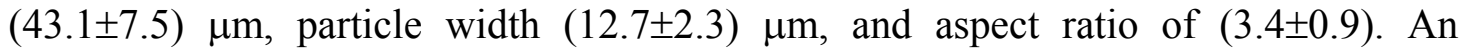

average of $0.7 \times 10^{6}$ particles $\left(1.4 \times 10^{6}\right.$ particles $\left./ \mathrm{mL}\right)$ were contained in the suspension for injection to the air pouch for both particle groups. The smaller particle group (group D) represented the particles in phagocytosable range for macrophage cells, and the particle size for larger particle group (group E) is too large to be internalized by macrophage cells.

Results of histological analysis shown in Figure 11.7 revealed that the pouch membranes stimulated with smaller particles (Group D) for $7 \mathrm{~d}$ averaged $(0.215 \pm 0.012) \mathrm{mm}$ in thickness, significantly thicker than pouches with larger particles (Group E) $((0.178 \pm 0.013) \mathrm{mm})$. It indicates that smaller particles in the phagocytosable range provoke higher response of macrophages. Green et al. [81] arrived at the same conclusions for spherical UHMWPE particles. 


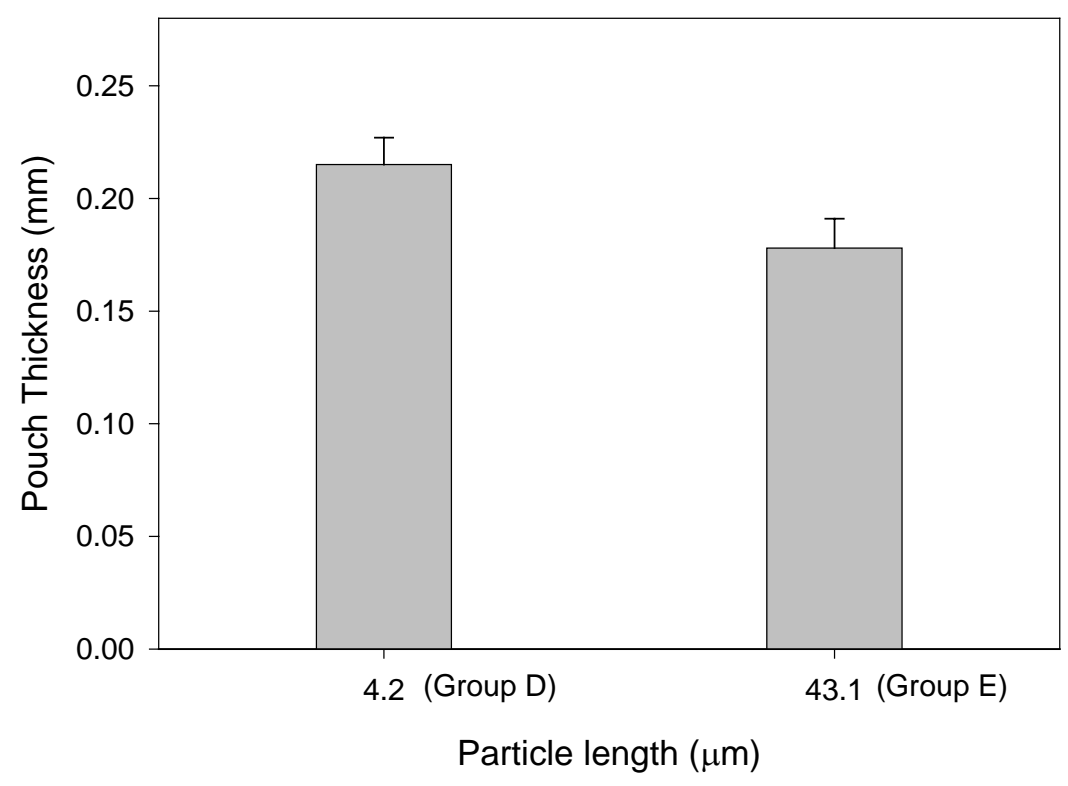

Figure 11.7 Comparison of pouch thickness for the murine air pouch tests with small (Group D) and larger (Group E) elongated UHMWPE particles.

\subsection{Effect of particle dosage}

Particle group D (elongated particles within phagocytosable range) mentioned in the previous sections were used in the air pouch tests. An average of $0.7 \times 10^{6}$ particles and $1.5 \times 10^{6}$ particles were contained in the suspension for injection to the air pouch to investigate the particle number effect on macrophage responses.

Figure 11.8 shows the results of histological analysis. The pouch membrane stimulated with $1.5 \times 10^{6}$ particles $\left(3.0 \times 10^{6}\right.$ particles $\left./ \mathrm{ml}\right)$ for $7 \mathrm{~d}$ averaged $(0.345 \pm 0.040) \mathrm{mm}$ in thickness, significantly thicker than pouches with $0.7 \times 10^{6}$ particles $\left(1.4 \times 10^{6}\right.$ particles $\left./ \mathrm{mL}\right)$ (pouch thickness $\left.=(0.215 \pm 0.012) \mathrm{mm}\right)$. It indicates that the large number of particles induce a stronger immunological responses by the macrophage activation. 


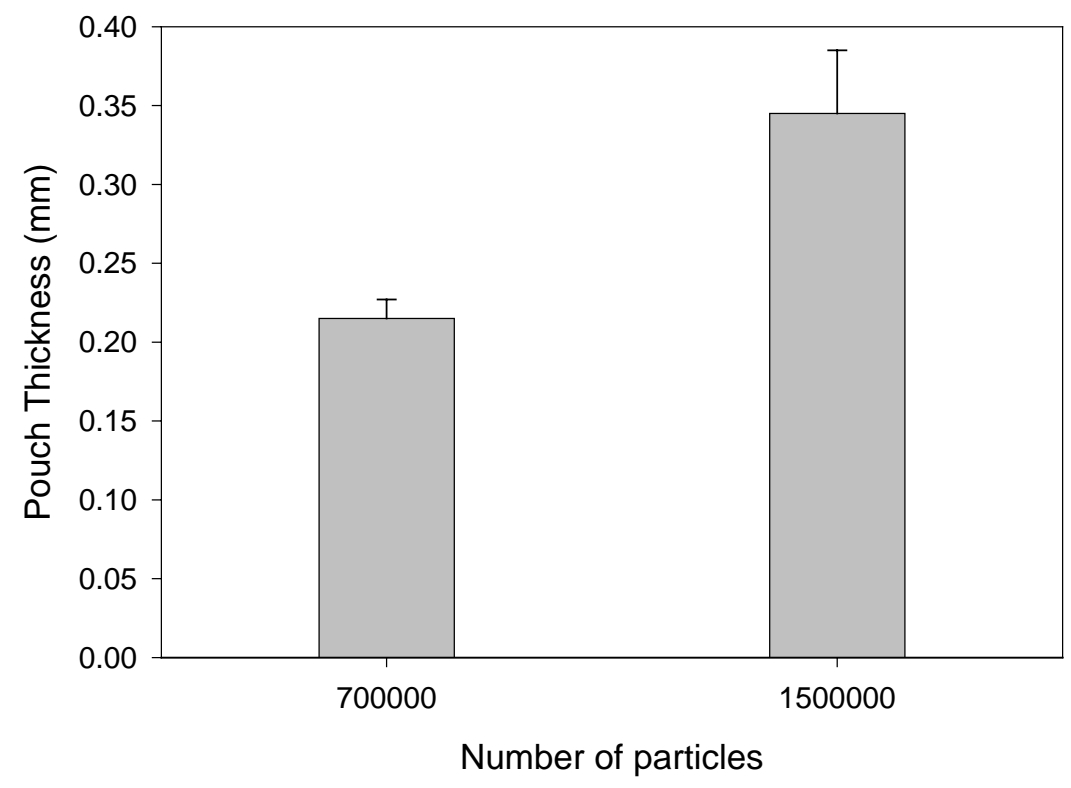

Figure 11.8 Comparison of pouch thickness for the murine air pouch tests with different number of particles (Group D, elongated particles within phagocytosable range)

\subsection{Combined effects from size, shape and dosage of particles}

The size of a particle can be expressed by the volume or the surface area of a particle as discussed in Chapter 10. The aspect ratio is used to characterize the shape of a particle in terms of the elongation of a particle. However, the size and shape of the particles are not independent variables. It is not possible to keep all other variables for particle size and shape the same while one variable is altered. For example, the change of the aspect ratio of a particle also leads to the change of the surface area of the particle when the particle volume is kept the same. 
In the previous sections 11.4-11.6 we mentioned the effects of particle size, shape and dosages effect on bioactivities. To further investigate the combined effects from particle size, shape and dosages, we shall compare the results of the bioactivity tests with candidate particle variables. Bioactivity results are plotted versus various particle descriptors that were introduced in Chapter 10. The purpose is to investigate the dominating factors leading to the difference of bioactivities induced by various UHMWPE particle populations. It is understood that the cytokine measurement of the air pouch experiments is sensitive to the measurement conditions and environments. There may exist more system errors of measurements from different batches. In this section, we integrate the bioactivity results from all the experiments. To reduce the system errors, the pouch membrane thickness is adopted as an indicator for the immunological responses.

From the viewpoint of chemical reaction, the total surface area of the particles is proportional to the total reaction sites available in the interaction processes between particles and macrophage cells. A plot of pouch membrane thickness versus the total surface area of the particles is shown in Figure 11.9. From the viewpoint of the digestion process by the macrophage cells, the volume of the particle is a candidate variable to affect the immunological responses. Thus Figure 11.10 shows a plot of the air pouch thickness versus the total volume of the particles applied in the murine air pouch experiments. 


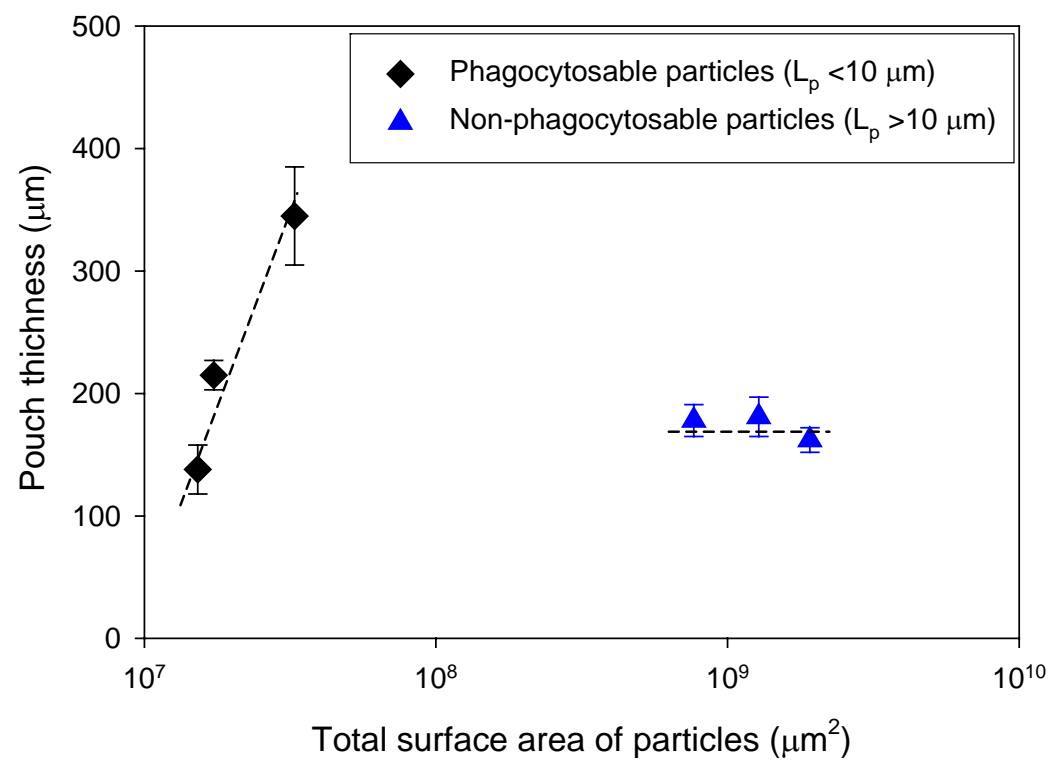

Figure 11.9 Plot of pouch thickness versus total surface area of particles.

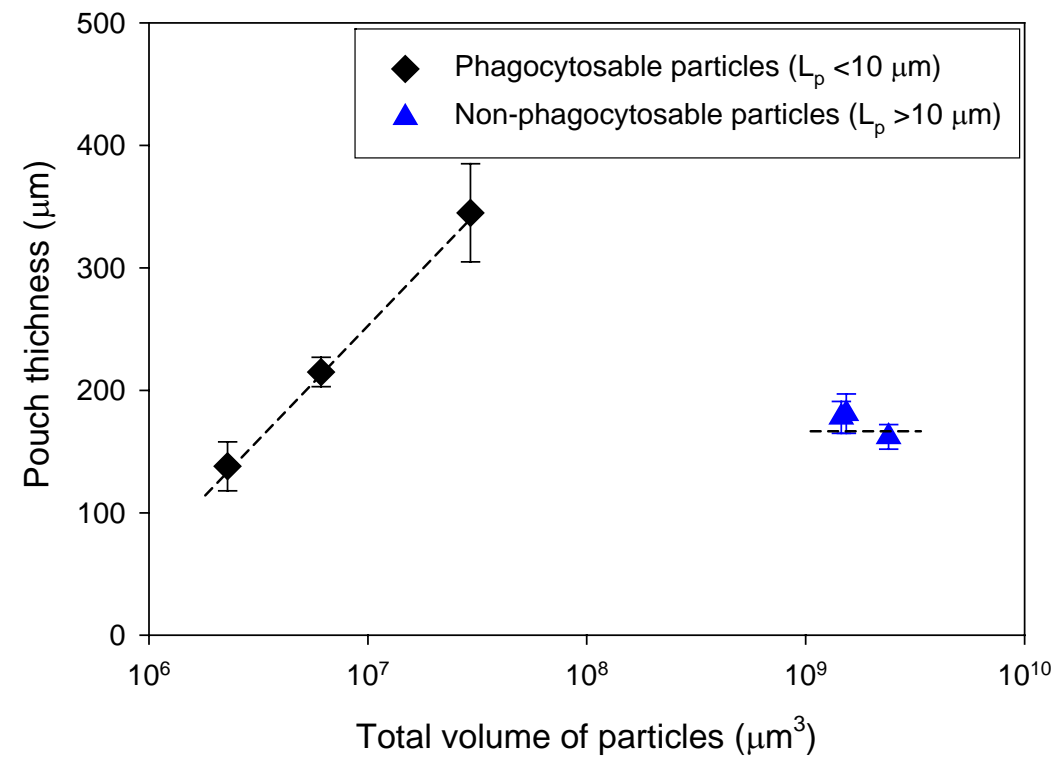

Figure 11.10 Plot of pouch thickness versus total volume of particles. 
From Figures 11.9-11.10, it is seen that for the smaller particles in the phagocytosable range (particle length smaller than $10 \mu \mathrm{m}$ ), the pouch thickness increases with increasing total surface area or volume of the particles. However, the pouch thickness decreases for the non-phagocytosable particles (particle length larger than $10 \mu \mathrm{m}$ ) even though the total surface area or volume is larger than the total surface area or volume of the phagocytosable particles. The pouch thickness induced by the non-phagocytosable particles with different total surface are or volume of particles did not show the significantly difference from our experimental results.

Results suggest that for the smaller particles in the phagocytosable range for macrophage cells, the immunological response (measured by pouch thickness) is stronger with larger total surface area or volume of the particles. Whether the surface area or the volume of the particle is the dominating factors cannot be clearly defined from these experimental results. When the particle is too large to be engulfed by the macrophage cells, the particle-macrophage interactions results in a weaker immunological response. It was observed that the macrophage cells tend to attach to the surface when the cell is not capable of engulfing the particle. Obviously the mechanisms of particle-macrophage interaction vary according to the critical particle size for phagocytosability of macrophage cells. This result suggests that the phagocytosis of particles induces a stronger bioactivity. The increase of the biological response is related to the quantity of total surface area or the volume of the particles applied to the murine air pouch tests. 
To further discuss the effect of particle shape on bioactivity, the pouch thickness was normalized by the total surface area or the total volume of the particles. The purpose is to focus on the particle-shape effect on the mechanisms of induced immunological response without the interference of the particle-dosage effects (total surface area and total volume of particles from the variance of the particle shape. Figure 11.11 are the plots of the normalized pouch thickness versus the aspect ratio of the particles. The results indicate that the phagocytosable particles induced stronger biological response also. It is also shown that the larger aspect ratio of particles stimulated stronger imuunological responses and resulted in a thicker pouch membrane from the plots. This applies to both phagocytosable and nonphagocytosable UHMWPE particles.

We think elongated particles have sharper edges. When the sharper edges get in contact with macrophage cells in the biological environments, higher mechanical stresses are applied on the cells by the particle edges. The higher mechanical stress might cause the damage of the membrane of the cells or further leads to the apotosis of the macrophage cells. The damage or death of the defense cells (macropahge cells) results in gathering more macrophage cells to fight the foreign materials (UHMWPE particles) and case a thicker pouch membrane. It also means a stronger immunological response is induced. 


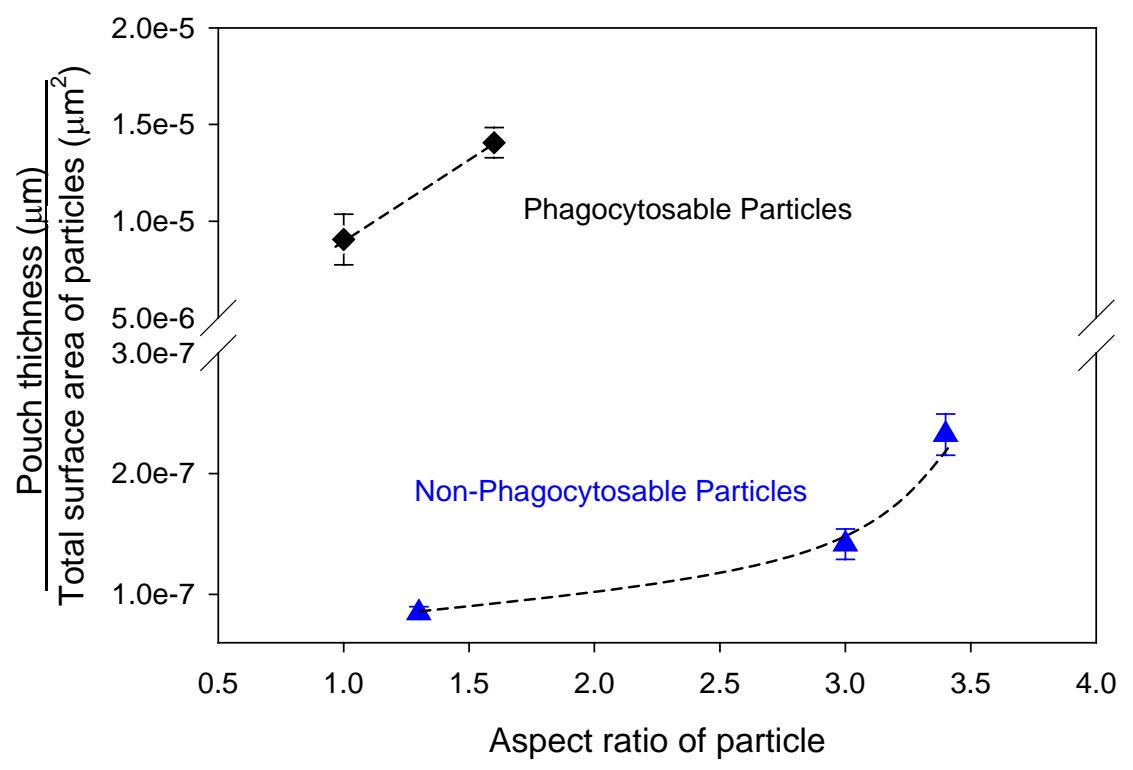

(a)

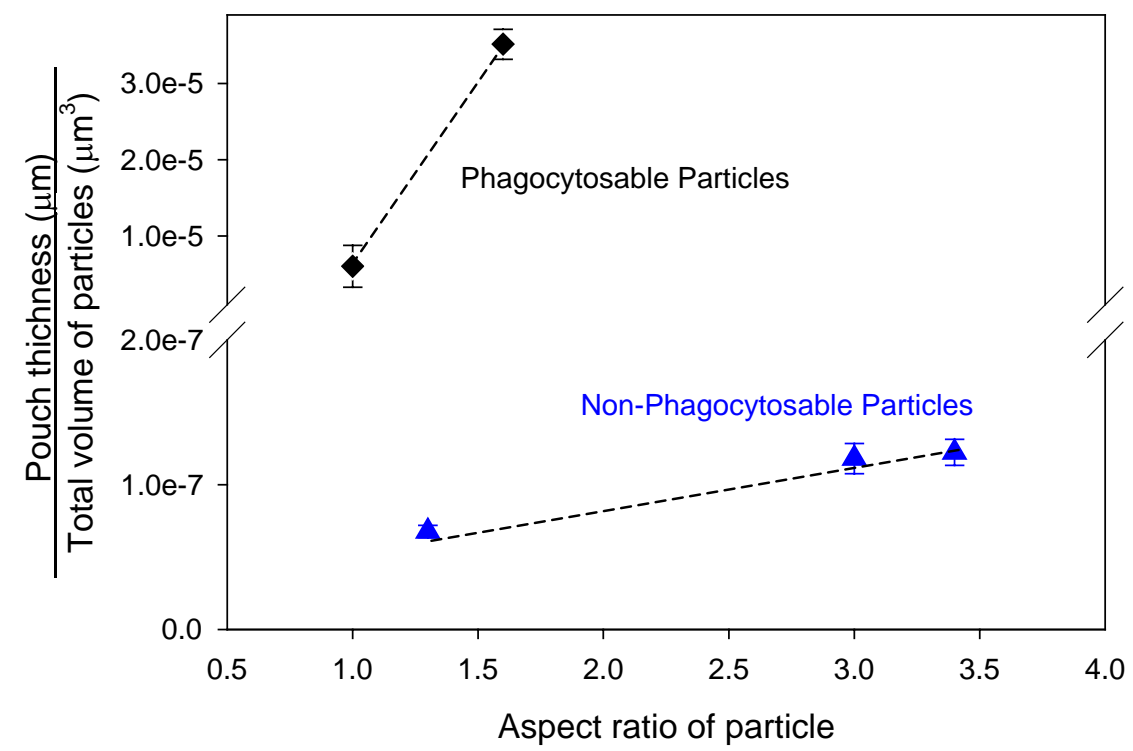

(b)

Figure 11.11 Plots of normalized pouch thickness versus aspect ratio of particles; (a) pouch thickness normalized by total surface area of particles; (b) pouch thickness normalized by total volume of particles. 


\subsection{Conclusion}

No one has investigated the effect of elongated UHMWPE particles that are more clinically relevant on bioactivity (interaction between macrophage cells and elongated glass fibers has been studied [125]). The effect of particle shape on bioactivity was first investigated by the murine air pouch experiments of UHMWPE particles in this collaborative study.

The results indicate that elongated particles induced stronger immunological responses while the particle volumes remain the same for particle populations. The results of particle-size effects show that the particles in the phagocytosable range induce larger macrophage activation than the larger particles do. These results from elongated particles are consistent with the conclusion we made in Chapter 10. 


\section{ChAPTER 12}

\section{SUMMARY AND CONCLUSIONS}

The objective of this study was to investigate the effect of UHMWPE particles on bioactivity. The critical questions addressed in Chapter 3 include:

- Are all particles bioactive?

- What is the effect of particle size?

- What is the effect of particle shape?

- What is the critical threshold concentration of a particle population with certain size and shape?

The following approaches were attempted: (1) Design of surface textures to generate narrowly distributed UHMWPE particles with controlled sizes and shapes; (2) Modeling of the particle-generation process and prediction of the generated UHMWPE particles; (3) Bioactivity tests of UHMWPE particles. Through these studies, we are able to provide most answers of the above questions. In the following sections, we will make conclusions from these approaches.

\subsection{Design of surface textures for particle generation}

Microfabrication techniques have been successfully applied to prepare the surface textures with controlled dimensions. A specific process has been developed to fabricate wedge features with defined dimensions on the silicon material. A narrowly 
distributed particle population was achieved when the uniformity of the surface textures was improved. The size of the generated UHMWPE particles covers the nonphagocytosable and phagocytosable particle populations from the viewpoint of phagocytosis of particles.

Conclusions of design of surface-textures are made as follows:

(1) Particle length is $\approx 0.8$ of the cutting-edge length.

(2) A minimum ratio of pitch distance to cutting-edge height $\left(D_{\mathrm{s}} / H_{\mathrm{c}}\right)$ of 14 is required for a clean micro-cutting process for UHMWPE particle generation.

(3) A larger normal load leads to a smaller aspect ratio of the particle.

(4) A larger cutting-edge height (under sufficient normal load to push the UHMWPE pin to the surface) leads to a smaller aspect ratio of the particle.

(5) A higher sliding speed results in a larger aspect ratio of the particle.

(6) We have successfully generated narrowly distributed UHMWPE particles in the range of $3 \mu \mathrm{m}$ to $43 \mu \mathrm{m}$.

\subsection{Modeling of UHMWPE particle generation}

Based on the observation of worn surfaces and wear particles of UHMWPE, a mechanism of the particle-generation process was proposed. It includes the following steps: (1) penetration of the cutting edge into the material, (2) accumulation of the deformed UHMWPW material in front of the cutting edge during the sliding process, (3) Lift-up of the cutting edge by the material resistant force, and (4) Formation of the 
particle. Model experiments were performed to investigate the movement of the cutting edge during the particle-generation process. The viscoelasticity, molecular orientation, and strain hardening of UHMWPE have also been taken into consideration. Following conclusions are made:

(1) In a nano-indentation test of UHMWPE, a $37.3 \%$ of the maximum penetration depth was recovered due to the viscoelasticity. Similar results were also obtained from a wedge-tip indentation test.

(2) Increase of material resistant force during the sliding process comes from strain hardening of UHMWPE and cumulative volume in front of the cutting face.

(3) Shear stress induces molecular orientation on the UHMWPE surface.

(4) Elastic modulus of UHMWPE surface is higher than the bulk material. It also means the surface layer of UHMWPE is harder than the bulk material.

(5) A smaller cutting-edge angle (a sharper edge) leads to a longer sliding distance of the tip. It is due to less material resistant force is distributed to the vertical direction to lift up the tip.

A mathematical model to predict the dimensions of generated UHMWPE particles was established. The calculation of penetration and sliding processes of the cutting edge was carried out by contact-mechanics principles. A correlation model based on the empirical results was also formulated. Conclusions are made as follows: 
(1) Initial penetration depth of the cutting edge can be calculated by $H_{\mathrm{p}}=\frac{W}{2 \cdot\left(C_{\mathrm{v}} \cdot Y\right) \cdot L_{\mathrm{c}} \cdot \tan \theta}$, where $C_{\mathrm{ep}}=\frac{1}{\sqrt{3}}\left[1+\ln \left(\frac{4}{3 \pi} \frac{E}{Y} \tan \left(\frac{\pi}{2}-\theta\right)\right]\right.$, and $C_{\mathrm{v}}=1.75 \cdot C_{\mathrm{ep}}$. It means the ratio of penetration depth predicted from the elastic-plastic assumption to the measured penetration depth is 1.75 .

(2) Iterative computing of the force balance during the sliding process can determine the displaced volume of UHMWPE during the sliding process. This displaced volume is projected to the volume of the particle generated.

(3) Correlation model has been established from the empirical results: $V_{\mathrm{p}}=39.5 \frac{L_{\mathrm{c}} \cdot H_{\mathrm{p}}{ }^{2} \cdot W^{0.6}}{V^{0.6}}($ unit: $\mu \mathrm{m}, \mathrm{N}, \mathrm{s})$

\subsection{Bioactivity of UHMWPE particles}

Murine air pouch animal tests have been used to test the biological responses induced by UHMWPE particles. Elongated particles that are commonly observed clinically were evaluated for the first time. Following conclusions are made from bioactivity studies of UHMWPE particles:

(1) Smaller particles within the phagocytosable range induce stronger bioactivity than large particles that cannot be engulfed by the macrophage cells.

(2) Elongated particles induce larger immunological responses. 
(3) The number of the particles with specified size and shape a macropahge cell can engulf (phagocytic capacity) can be estimated from a biophysical approach of the phagocytosis process.

(4) For phagocytosbale particles, when the number of the particles is less than phagocytic capacity, the immunological response is higher with increasing number of particles.

(5) When the number of particles exceeds the phagocytic capacity, the excess particles can be treated as non-phagocytosable particles. Thus the induced bioactivity remains about the same. 


\section{CHAPTER 13}

\section{FUTURE WORK AND RECOMMENDATIONS}

\subsection{Improvements of current techniques of particle generation}

There is lack of sub-micrometer particles in this study. We need to further generate particles in this range for bioactivity tests. We have demonstrated the feasibility of generating UHMWPE wear particles with different shapes by surfacetexturing techniques. The main issue is the size of the particles. Since sub-micrometer round particles and micron-sized elongated particles were observed from clinical retrieval studies, the question is whether this technique is capable of producing different sizes and shapes in the sub-micrometer range. The current microfabrication technique is capable of producing features with a $0.1 \mu \mathrm{m}$ resolution; hence, theoretically there is no barrier to produce particles down to $0.1 \mu \mathrm{m}$ size range. We have attempted to produce particles in the sub-micrometer range but the rate of producing such particles is extremely low and the particle-size distribution is much wider than expected. We attribute this result to our lack of understanding of the viscoelastic response of UHMWPE. Theoretically, there exists a minimum penetration depth the cutting edge should reach in order to induce an effective microcutting process. More efforts are needed to identify the critical parameters to produce sub-micrometer particles with different shapes. 
The current technique produces particles with precisely controlled size and shape, but the particle generation rate is low $(\approx(0.8$ to 1$) \mathrm{mg} / \mathrm{d})$. Efforts to increase the particle-generation rate are needed. The strategy may include changing different contact geometries, speeds, and loads. However, the trade-off might be the narrowness of the particle-size distribution.

\subsection{Mechanism study for osteolysis induced by particles}

More bioactivity tests for UHMWPE particles with various sizes and shapes are needed, especially for the particles in the submicron ranges. For a specific size and shape of the particle population, the experiments should be further carried out with controlled total surface areas, total volumes, and number of the particle populations independently. Then the boundaries of the biological responses induced by different mechanisms can be further identified.

More efforts should be focused on the biochemical pathway of osteolysis induced by UHMWPE particles and the gene expression of the cells during the process. The availability of UHMWPE particle with various sizes and shapes has opened an entrance to study the mechanism. Once the detailed mechanism of osteolysis induced by particles is discovered, the early detection of the aseptic loosening becomes possible. Then the development of the medical treatment to block the pathway and to prevent osteolysis will be feasible. Thus the life of the total joint replacement can be further prolonged. 


\section{REFERENCES}

1. Wright, T. M., and Goodman, S. B. Implant Wear in Total Joint Replacement: Clinical and Biologic Issues, Material and Design Considerations, published by American Academy of Orthopaedic Surgeons, pp. 3-11, 2001.

2. Dowson, D. Medical engineering- the multi-disciplinary challenge. Proc. Instn. Mech. Engrs., 205, pp. 1-10, 1991.

3. Cheal, E. J., Spector, M., and Hayes, W. Role of Loads and Prosthesis Material Properties on the Mechanics of the Proximal Femur After Total Hip Arthroplasty. Journal of Orthopaedic Research, 10, pp. 405-422, 1992.

4. Evans, S. L., and Gregson, P. J. Numerical optimization of the design of a coated cementless hip prosthesis. Journal of Materials Science: Materials in Medicine, 5, pp. 507-510, 1994

5. Toni, A., McNamara, B., Viceconti, M., Sudanese, A., Baruffaldi, F., and Giunti, A. Bone Remodelling after Total Hip Arthroplasty. Journal of Materials Science: Materials in Medicine, 7, pp. 149-152, 1996.

6. Weinans, H., and Huiskes, R. Effects of Fit and Bonding Characteristics of Femoral Stems on Adaptive Bone Remodeling. Journal of Biomechanical Engineering, 116, pp. 393-400, 1994.

7. Kurtz SM, Muratoglu OK, Evans M, Edidin AA. Advances in the processing, sterilization, and crosslinking of ultra-high molecular weight polyethylene for total joint arthroplasty. Biomaterials, 20. pp. 1659-1688, 1999. 
8. McKellop H, Shen FW, Lu B, Campbell P, Salovey R. Development of an extremely wear-resistant ultra high molecular weight polyethylene for total hip replacements. J Orthop Res, 17, pp. 157-167, 1999.

9. Muratoglu OK, Bragdon CR, O'Connor DO, Jasty M, Harris WH. A novel method of cross-linking ultra-high-molecular-weight polyethylene to improve wear, reduce oxidation, and retain mechanical properties. J Arthroplasty, 16, pp. 149-160, 2001.

10. A Consumer's Guide to Total Joint Replacement, Supplement to USA Today, February 5, 1999.

11. Fisher, J., and Dowson, D. Tribology of total artificial joints. Proc. Instn. Mech. Engrs., 205, pp. 73-79, 1991.

12. Charnley, J. Low Friction Principle. Low Friction Arthroplasty of the Hip: Theory and Practice. Eds. Berlin, Published by Springer-Verlag, 1979.

13. Masuda, S., Kitano, H., Ikeada, J., Yoshihara, Y., Mukai, K., Shimotoso, T., and Noda, I. New Approach to a Cementless Ceramic Component for Artificial Knee Joint. Key Engineering Materials, 218-220, pp. 577-580, 2002.

14. Zhou, Y. S., Ikeuchi, K., and Ohashi, M. Comparison of the friction properties of four ceramic materials for joint replacements. Wear, 210, pp. 171-177, 1997.

15. Lancaster, J. G., Dowson, D., Issac, G. H., Fisher, J. Wear of ultra-high molecular weight polyethylene sliding on metallic and ceramic counterfaces representative of current femoral surfaces in joint replacement. Proceedings of 
the Institution of Mechanical Engineers, Part H: Journal of Engineering in Medicine, 211, pp. 17-24, 1997.

16. Park, S-H., McKellop, H., Lu, B., Chan, F., and Chiesa, R. Wear morphology of metal-metal implants: hip simulator tests compared with clinical retrievals, ASTM Special Technical Publication, 1346, pp. 129-143, 1998.

17. Chan, F., Medley, J. B., Bobyn, J. D., and Krygier, J. J. Numerical analysis of time-varying fluid film thickness in metal-metal hip implants in simulator tests, ASTM Special Technical Publication, 1346, pp. 111-128, 1998.

18. Furman, B. R., and Saha, S. Mechanical properties of bone cement as controlled by processing technique: a critical review of the literature. Southern Biomedical Engineering Conference - Proceedings, pp. 301-304, 1997.

19. Rosenstein, A., McDonald, W., Iliadis, A., McLardy-Smith, P. Revision of cemented fixation and cement-bone interface strength. Proceedings of the Institution of Mechanical Engineers, Part H: Journal of Engineering in Medicine, 206, pp. 47-49, 1992.

20. Bostrom, M. P., Bennett, A. P., Rimnac, C. M., and Wright, T. M. The Natural History of Ultra High Molecular Weight Polyethylene. Clinical Orthopaedics and Related Research, No. 309, pp. 20-28, 1994.

21. Edidin, A. A., and Kurtz, S. M. The influence of mechanical behavior on the wear of four clinically relevant polymeric biomaterials in a hip simulator. Journal of Arthroplasty, 15, pp.321-331, 2000. 
22. Farrar, D. F., and Brain, A. A. The microstructure of ultra-high molecular weight polyethylene used in total joint replacements. Biomaterials, 18, pp. 1677-1685, 1997.

23. ASTM F648-00 Standard Specification for Ultra-High-Molecular-Weight Polyethylene Powder and Fabricated Form for Surgical Implants, ASTM International, 2003.

24. ISO standard 5834-1 Implants for surgery: Ultra-high molecular weight polyethylene -- Part 1: Powder form, ISO Standards, 1998.

25. Han, K. S., Wallace, J. F., Truss, R. W., and Geil, P. H. Powder compaction, sintering, and rolling of ultra-high molecular weight polyethylene and its composites. Journal or Macromolecular Science-Phys, B19, pp. 313-349, 1981.

26. Fischer, D. A., Sambasivan, S., Shen, M. C., and Hsu, S. M. Wear induced molecular orientation in UHMWPE measured by soft x-ray absorption. Transactions of Society for Biomaterials, 22, pp. 351, 1999.

27. Sambasivan, S., Fischer, D. A., Shen, M. C., and Hsu, S. M. Effects of wear motion on UHMWPE molecular orientation. Transactions of Society for Biomaterials, 22, pp. 194, 1999.

28. Sambasivan, S., Fischer, D. A., Shen, M. C., Tesk, J. A., and Hsu, S. M. Effects of annealing on UHMWPE molecular orientation. Transactions of the $6^{\text {th }}$ World Biomaterials Congress, pp. 178, 2000. 
29. Wang, A., Sun, D. C., Yau, S.-S., Edwards, B., Sokol, M., Essner, A., Polineni, V. K., and Stark, C. Orientation softening in the deformation and wear of ultrahigh molecular weight polyethylene. Wear, 203-204, pp. 230-241, 1997.

30. Bostrom, M. P., Bennett, A. P., Rimnac, C. M., and Wright, T. M. The Natural History of Ultra High Molecular Weight Polyethylene. Clinical Orthopaedics and Related Research, No. 309, pp. 20-28, 1994.

31. Harris, W. H. The problem is osteolysis. Clinical Orthopaedics and Related Research, 311, pp. 46-53, 1995.

32. Amstutz, H. C., Campbell, P., Kossovsky, N., and Clarke, I. C. Mechanism and clinical significance of wear debris-induced osteolysis. Clinical Orthopaedics and Related Research, 276, pp. 7-18, 1992.

33. Maloney, W. J., and Smith, R. L. Periprosthetic osteolysis in total hip arthroplasty: the role of particulate wear debris. Journal of Bone and Joint Surgery, 77A, pp. 1448-1461, 1995.

34. Wright, T. M., Rimnac, C. M., Stulberg, S. D.; et al. Wear of polyethylene in total joint replacements. Observations form retrieved PCA knee implants. Clinical Orthopaedics and Related Research, 276, pp. 126-134, 1992.

35. Wolfarth, D. L., Han, D. W., Bushar, G., and Parks, N. L. Separation and characterization of polyethylene wear debris from synovial fluid and tissue samples of revised knee replacements. Journal of Biomedical Materials Research, 34, pp. 57-61, 1997. 
36. Campbell, P., Ma, S., Yeom, B., McKellop, H., Schmalzried, T. P., and Amstutz, H. C. Isolation of predominantly submicron-sized UHMWPE wear particles from periprosthetic tissues. Journal of Biomedical Materials Research, 29, pp. 127-131, 1995.

37. Shanbhag, A. S., Jacobs, J. J., Glant, t. T., Gilbert, J. L., Black, J., and Galante, J. O. Composition and morphology of Wear debris in failed uncemented total hip replacement. Journal of Bone and Joint Surgery, 76-B, pp. 60-67, 1994.

38. Hailey, J. L., Ingham, E., Stone, M., Wroblewski, B. M., and Fisher, J. Ultrahigh molecular weight polyethylene wear debris generated in vivo and in laboratory tests; the influence of counterface roughness. Proc. Instn. Mech. Engrs., 210, pp. 3-10, 1995.

39. Lee, J-M., Salvati, E. A., Betts, F., Dicarlo, E. F., Doty, S. B., and Bullough, P. G. Size of metallic and polyethylene debris particles in failed cemented total hip replacements. The Journal of Bone and Joint Surgery, 74-B, pp. 380-384, 1992.

40. Maloney, W. J., Smith, R. L., Schmalzried, T. P., Chiba, J., Huene, D., and Rubash, H. Isolation and characterization of wear particles generated in patients who have had failure of a hip arthroplasty without cement. The Journal of Bone and Joint Surgery, 77-A, No. 9, pp. 1301-1310, 1995.

41. Schmalzried, T. P., Campbell, P., Schmitt, a. K., Brown, I. C., and Amstutz, H. C. Shapes and dimensional characteristics of polyethylene wear particles generated in vivo total knee replacements compared to total hip replacements. Journal of Biomedical Materials Research, 38, pp. 203-210, 1997. 
42. Hirakawa, K., Bauer, T. W., Stulberg, B. N., and Wilde, A. H. Comparison and quantification of wear debris of failed total hip and total knee arthroplasty. Journal of Biomedical Materials Research, 31, pp. 257-263, 1996.

43. Bauer, T. W. Indentification of Orhopaedic Wear Debris. The Journal of Bone and Joint Surgery, 78-4, No. 4, pp. 479-481, 1996.

44. Besong, A. A., Tipper, J. L., Ingham, E., Stone, M. H., Wroblewski, B. M., Fisher, J. Quantitative comparison of wear debris from UHMWPE that has and has not been sterilized by gamma irridiation. The Journal of Bone and Joint Surgery, 80-B, pp. 340-344, 1998.

45. Doorn, P. F., Campbell, P. A., Worrall, J., Benya, P. D., McKellop, H. A., and Amstutz, H. C. Metal wear particle characterization from metal on metal total hip replacements: transmission electron microscopy study of periprosthetic tissues and isolated particles. Journal of Biomedical Materials Research, 42, pp. 103-111, 1998.

46. Kobayashi, A., Bonfield, W., Kadoya, Y., Yamac, T., Freeman, M. A. R., Scott, G., and Revell, P. A. The size and shape of particulate polyethylene wear debris in total joint replacements. Proc. Instn. Mech, Engrs, 211, pp. 11-15, 1997.

47. Landry, M. E., Blanchard, C. R., Mabrey, J., Wang, X., Agrawal, C. M. Morphology of In Vitro Generated Ultrahigh Molecular Weight Polyethylene Wear Particles as a Function of Contact Conditions and Material Parameters. Journal of Miomedical materials Research (Applied Biomaterials), 48, pp. 61-69, 1999. 
48. Lewis, G. Polyethylene wear in total hip and knee arthroplasties. Journal of Biomedical Materials Research (Appl Biomater), 38, pp.55-75, 1997.

49. Mabrey, J. d., Afsar-Keshmiri, a., McClung, G. A., Pember, M. A., Wooldridge, M., Agrawal, C. M. Comparison of UHMWPE Particles in Synovial Fluid and Tissues from Failed THA. Journal of Biomedical Materials Research, 58, pp. 196-202, 2001.

50. Mosleh, M., and Suh, N. P. Wear particles of polyethylene in biological systems. Tribology Transactions, 39, pp.843-848, 1996.

51. Ruben, G. C., Blanchet, T. A., Kennedy, F. E. Formation of UHMWPE polymeric transfer films on sliding glass counterfaces: early and steady-state wear studied by transmission electron microscopy. Journal of Material Science, 28(4), pp. 1045-1058, 1993.

52. Willert, H.-G., and Semlitsch, M. Reactions of the articular capsule to wear products of artificial joint prostheses. Journal of Biomedical Materials Research, 11, pp. 157-164, 1977.

53. Basle, M. F., Bertrand, G., Guyetant, S., Chappard, D., and Lesourd, M. Migration of metal and polyethylene particles from articular prostheses may generate lymphadenopathy with histiocytosis. Journal of Biomedical Materials Research, 30, pp. 157-164, 1996.

54. Schmalzried, T. P., Jasty, M., and Harris, W. H. Periprosthetic bone loss in total hip arthroplasty. The Journal of Bone and Joint Surgery, Vol. 74-A, pp. 849-863, 1992. 
55. Murray, D. W., and Rushton, N. Macrophages stimulate bone resorption when they phagocytose particles. The Journal of Bone and Joint Surgery, 72-B, pp. 988-992, 1990.

56. Goodman, S. B., Huie, P., Song, Y., Schurman, D., Maloney, W., Woolson, S., and Sibley, R. Cellular Profile and Cytokine Production at Prosthetic Interfaces. The Journal of Bone and Joint Surgery, Vol. 80-B, pp. 531-539, 1998.

57. Granchi, D., Verri, E., Ciapetti, G., Stea, S., Savarino, L., Sudanese, A., Mieti, M., Rotini, R. Dallari, D., Zinghi, G., and Montanaro, L. Bone-resorbing Cytokines in Serum of Patients with Aseptic Loosening of Hip Prostheses. The Journal of Bone and Joint Surgery, Vol. 80-B, pp. 912-917, 1998.

58. Clarke, I. C. Wear of artificial joint materials IV: hip joint simulator studies. Engineering in Medicine, 10, No. 4, pp. 189-198, 1981.

59. Davidson, J. A., and Schwartz, G. Wear, Creep, and Frictional Heat of Femoral Implant Articulating Surfaces and the Effect on Long-Term Performance-Part I, A Review. Journal of Biomedical Materials Research: Applied Biomaterials, vol. 21, No. A3, pp. 261-285, 1987.

60. Davidson, J. A., and Schwartz, G., and Lynch, G. Wear, Creep, and Frictional Heat of Femoral Implant Articulating Surfaces and the Effect on Long-Term Performance-Part II, Friction, Heating, and Torque. Journal of Biomedical Materials Research: Applied Biomaterials, vol. 21, No. A3, pp. 69-91, 1988. 
61. Davidson, J. A., Gir, S., and Paul, J. P. Heat Transfer Analysis of Frictional Heat Dissipation During Articulation of Femoral Implants. Journal of Biomedical Materials Research: Applied Biomaterials, vol. 22, No. A3, pp. 281-309, 1988.

62. Humphreys, P. K., Orr, J. F., and Bahrani, A. S. Testing of total hip replacements: endurance tests and stress measurements Part 1: endurance tests. Proc. Instn. Mech. Engrs., 204, pp. 29-34, 1990.

63. Humphreys, P. K., Orr, J. F., and Bahrani, A. S. Testing of total hip replacements: endurance tests and stress measurements Part 2: stress measurements. Proc. Instn. Mech. Engrs., 204, pp. 35-41, 1990.

64. Saikko, A. A simulator study of friction in total replacement hip joints. Proc. Instn. Mech. Engrs., 206, pp. 201-211, 1992.

65. Saikko, V., Pavvolainen, P., Kleimola, M., and Slatis, P. A five-station hip joint simulator for wear rate studies. Proc. Instn. Mech. Engrs., 206, pp. 195-200, 1992.

66. Tateishi, T., Hyodo, K., Kondo, K., and Miura, K. Simulator test of artificial joints. Materials Science and Engineering, pp. 121-125, 1994.

67. Bergmann, G., Graichen, F., Rohlmann, A. Hip joint loading during walking and running, measured in two patients. Journal of Biomechanics, 26(8), pp. 969-990, 1993.

68. Paul, J. P. Forces transmitted by joints in the human body. Proc. Inst. Mech. Engr. 181, pp. 8-15, 1966. 
69. Crowninshield, R. D., Johnston, R. C., Andrews, J. G., and Brand, R. A. A biomechanical investigation of the human hip. Journal of Biomechanics, 11, pp. 75-78, 1978.

70. Kilvington, M., Goodman, R. M. F. In vivo hip joint forcese recorded on a strain gauged 'English' prosthesis using an implanted transmitter. Engineering in Medicine, 10(4), pp. 175-187, 1981.

71. Rydell, N. W. Forces acting in the femoral head prosthesis. Acta. Ortho. Scand (suppl.) 88, p. 37, 1966.

72. Kotzar, G. M., Davy, D. T., Goldberg, V. M., Heiple, K. G., Berilla, J., Heiple, K. G., Brown, R. H., and Burstein, A. H. Telemeterized in vivo hip joint force data: a report on two patients after total hip surgery. Journal of Orthopaedic Research, 9, pp. 621-633, 1991.

73. Ramakrishnan, H. K., Kadaba, M. P. On the estimation of joint kinematics during gait. Journal of Biomechanics, 24(10), pp. 969-977, 1991.

74. Wang, A., Sun, D. C., Yau, S-S., Edwards, B., Sokol, M., Essner, A., Polineni, V. K., Stark, C., and Dumbleton, J. H. Orientation softening in the deformation and wear of ultra-high molecular weight polyethylene. Wear, 203-204, pp. 230$241,1997$.

75. Wang, A., Polineni, V. K., Essner, A., Sokol, M., Sun, D. C., Stark, C., and Dumbleton, J. H. The significance of nonlinear motion in the wear screening of orthopaedic implant materials. Journal of Testing and Evaluation, pp. 239-245, 1997. 
76. Wang, A., Sun, D. C., Stark, C., and Dumbleton, J. H. Wear mechanisms of UHMWPE in total joint replacements. Wear, 181-183, pp. 241-249, 1995.

77. Shen, M. C., Hsu, S. M., Tesk, J. A., Christou, A. A novel multiaxial wear tester for accerated testing of materials. Transactions of Orthopedic Research Society, 24, pp, 852, 1999.

78. Allen, M., Brett, F., Millett, P., and Rushton, N. The effects of particulate polyethylene at a weight bearing bone-implant interface. The Journal of Bone and Joint Surgery, 78-B, pp. 32-37, 1996.

79. Catelas, I., Huk, O. L., Petit, A., Zukor, D. J., Marchand, R., and Yahia, L. Flow cytometric analysis of macrophage response to ceramic and polyethylene particles: effects of size, concentration, and composition. Journal of Biomedical Materials Research, 41, pp. 600-607, 1998.

80. Goodman, S. B., Davidson, J. A., Song, Y., Martial, N., and Fornasier, V. L. Histomorphological reaction of bone to different concentrations of phagocytosable particles of high-density polyethylene and Ti-6Al-4V alloy in vivo. Biomaterials, 17, pp. 1943-1947, 1996.

81. Green, T. R., Fisher, J., Stone, M., Wroblewski, B. M., and Ingham, E. Polyethylene particles of a 'critical size' are necessary for the induction of cytokines by macrophages in vitro. Biomaterials, 19, pp. 2297-2302, 1998.

82. Horowitz, S. M., and Gonzales, J. B. Effect of polyethylene on macrophages. Journal of Orthopaedics Research, 15, pp. 50-56, 1997. 
83. Howie, D. W., Vernon-Roberts, B., Oakeshott, R., and Manthey, B. A rat model of resorption of bone at the cement-bone interface in the presence of polyethylene wear particles. The Journal of Bone and Joint Surgery, 70-A, pp. 257-263, 1988.

84. Martinez, M. E., Medina, S., del Campo, M. T., Garcia, J. A., Rodrigo, a., and Munuera, L. Effect of polyethylene particles on human osteoblastic cell growth. Biomaterials, 19, pp. 183-187, 1998.

85. Murray, D. W., and Rushton, N. Macrophages stimulate bone resorption when they phagocytose particles. The Journal of Bone and Joint Surgery, 72-B, pp. 988-992, 1990.

86. Shanbhag, A. S., Jacobs, J. J., Black, J., Galante, J. O., and Glant, T. T. Macrophage/particle interactions: effect of size, composition, and surface area. Journal of Biomedical Materials Research, 28, pp. 81-90, 1994.

87. Voronov, I., Santerre, J. P., Hinek, A., Callahan, J. W., Sandhu, J., and Boynton, E. L. Macrophage phagocytosis of polyethylene particulate in vitro. Journal of Biomedical Materials Research, 39, pp. 40-51, 1998.

88. Stachowiak, G. W. Numerical characterization of wear particles morphology and angularity of particles and surfaces. Tribology International, 31, pp. 139-157, 1998.

89. Stachowiak, G. W., Stachowiak, G. B., and Campbell, P. Application of numerical descriptors to the characterization of wear particles obtained from joint replacements. Proc. Instn. Mech. Engrs, 211, Part H, pp. 1-10, 1997. 
90. Marcus, K., and Allen, C. The sliding wear of ultrahigh molecular weight polyethylene in an aqueous environment. Wear, 178, pp. 17-28, 1994.

91. Mosleh, M., and Suh, N. P. Wear particles of polyethylene in biological systems. Tribology Transactions, 39, pp.843-848, 1996.

92. Saikko, A. Wear and friction properties of prosthetic joint materials evaluated on a reciprocating pin-on-flat apparatus. Wear, 166, pp. 169-178, 1993.

93. Barrett, T. S., Stachowiak, G. W., and Batchelor, A. W. Effect of roughness and sliding speed on the wear and friction of ultra-high molecular weight polyethylene. Wear, 153, pp. 331-350, 1992.

94. Shanbhag, A. S., Hasselman, C. T., and Rubash, H. E. Technique for generating submicron ultra high molecular weight polyethylene particles. Journal of Orthopaedic Research, 14, pp. 1000-1004, 1996.

95. Yarovoy, Y. K., Baran, G., Wunder, S. L., Wang, R. Submicron-Size Particles of Ultrahigh Molecular Weight Polyethylene Produced via Nonsolvent and Temperature-Induced crystallization. Journal of Biomedical Materials Research (Applied Biomaterials), 53, pp. 152-160, 2000.

96. Madou, M. Fundamentals of Microfabrication, published by CRC, 1997.

97. Lee, B., Demroff, H. P., Drew, M. M., Elliott, T. S., Mazumdar, T. K., McIntyre, P. M., Pang, Y., Smith, D. D., and Trost, H.-J. Knife-edge thin film field emission cathodes. IEEE, pp. 2705-2707, 1993.

98. Briscoe, B. J., Fiori, L., and Pellilo. Nano-indentation of polymeric surfaces. J. Phys. D.: Appl. Phys., 31, pp. 2395-2405, 1998. 
99. Hill, R. M., and Dissado, L. A. Relaxation in elastic and viscoelastic materials. Journal of Materials Science, 19, pp. 1576-1595, 1983.

100. Gauthier, C., Schirrer, R. Time and temperature dependence of the scratch properties of poly(methylmethacrylate) surfaces. Journal of Materials Science, 35, pp. 2121-2130, 2000.

101. Gauthier, C., Lafaye, S., Schirrer, R. Elastic recovery of a scratch in a polymeric surface: experiments and analysis. Tribology International, 34, pp. 469-479, 2001.

102. Jardret, V., Zahouani, H., Loubet, J. L., Mathia, T. G. understanding and quantification of elastic and plastic deformation during a scratch test. Wear, 218, pp. 8-14, 1998.

103. Xiang, C., Sue, H.-J., Chu, J., Coleman, B. Scratch Behavior and Material Property Relationship in Polymers. Journal of Polymer Science: Part B: Polymer Physics, 39, pp. 47-59, 2001.

104. Kurtz, S. M., Pruitt, L., Jewett, C. W., Crawford, R. P., Crane, D. J., and Edidin, A. A. The yielding, plastic flow, and fracture behavior of ultra-high molecular weight polyethylene used in total joint replacements. Biomaterials, 19, pp. 19892003, 1998.

105. Krzypow, D. J., and Rinmac, C. M. Cyclic steady state stress-strain behavior of UHMWPE. Biomaterials, 21, pp. 2081-2087, 2000.

106. Kurtz, S. M., Rimnac, C. M., and Bartel, D. L. Predictive model for tensile true stress-strain behavior of chemically and mechanically degraded UHMWPE. 
Journal of Biomedical Materials Research (Applied Biomaterials), 43, pp. 241248, 1998.

107. G'Shell, C., and Jonas, J. J. Determination of the plastic behavior of solid polymers at constant true strain rate. Journal of Material Science, 14, pp. 583, 1979.

108. Briscoe, B. J., Pelillo, E., and Sinha, S. K. Scratch hardness and deformation maps for polycarbonate and polyethylene. Polymer Engineering and Science, 36, 24, pp. 2996-3005, 1996.

109. Briscoe, B. J., Evans, P. D., Pelillo, E., and Sinha, S. K. Scratching maps for polymers. Wear, 200, pp. 137-147, 1996.

110. Schey, J. A. Triboloy in metalworking- friction, lubrication and wear, Published by American Society for Metals, 1984.

111. Hill, R., Lee, E. H., and Tupper, Theory of wedge indentation of ductile metals, Proceedings of the Royal Society, A188, pp. 273-289, 1947.

112. Yu, W., and Blanchard, J. P. An elastic-plastic indentation model and its solutions. Journal of Materials Research, Vol. 11, No. 9, pp. 2358-2367, 1996.

113. Johnson, K. L. The correlation of indentation experiments. Journal of Mechanics and Physics of Solids, 18, pp. 115-126, 1970.

114. Aderem, A., Underhill, D. M. Mechanisms of phagocytosis in macrophages Annu. Rev. immunol. 17, pp. 593-623, 1999. 
115. Besterman, J. M., Airhart, J. A., and Low, R. B. Macrophage Phagocytosis: analysis of particle binding and internalization. American Journal of Physiology, pp. C 339-346, 1982.

116. Bos, H., de Souza, W. Morphometrical Method for Estimating Mean Cell Volume of Phagocytosing Cells. Microscopy and Microanalysis, 7, pp. 39-47, 2001.

117. Bowers, B., Olszewski, T. E., Hyde, J. Morphometic Analysis of volumers and Surface Areas in Membrane Compartments during Endocytosis in Acanthamoeba. Journal of Cell Biology, 88, pp. 509-515, 1981.

118. Simon, S. I., Schmid-Schonbein, G. W. Biophysical aspects of microsphere engulfment by human neutrophils. Biophysical Journal, 53, pp. 163-173, 1988.

119. Schmid-Schonbein, G. W., Shih, Y. Y., and Chien, S. Morphometry of Human Leukocytes. Blood, Vol. 56, No. 5, pp. 866-876, 1980.

120. Schroeder, F., Kinden, D. A. Measurement of phagocytosis using fluorescent latex beads. Journal of Biochemical and Biophysical Methods, 8, pp. 15-27, 1983.

121. Wooley, P. H., Song, Z., Mayton, L., Nasser, S., Cho, U., Shen, M., and Hsu, S. Variations in UHMWPE particle morphology result in different cellular and cytokine responses in the murine air pouch model. Proceeding of $6^{\text {th }}$ World Biomaterials Congress, p. 379, 2000. 
122. Hooper, K. A., Nickolas, T. L., Yurkow, E. J., Kohn, J., Laskin, D. L. Characterization of the inflammatory response to biomaterials using a rodent air pouch model. Journal of Biomedical Material Research, 50. pp. 365-74, 2000.

123. Edwards, J.C., Sedgwick, A. D., Willoughby, D. A. The formation of a structure with the features of synovial lining by subcutaneous injection of air: an in vivo tissue culture system. J Pathology, 134, pp.147-56, 1981.

124. Yang, S. Y., Ren, W., Park, Y., Sieving, A., Hsu, S., Nasser, S., and Wooley, P. H. Diverse cellular and apoptotic responses to variant shapes of UHMWPE particles in a murine model of inflammation. Biomaterials, 23, pp. 3535-3543, 2002.

125. Blake, T., Castranova, V., Schwegler-Berry, D., Baron, P., Deye, G. J., Li, G., and Jones, W. Effect of fiber length on glass microfiber cytotoxicity, Journal of Toxicity and Environment Health, Part A, 54, pp. 243-159, 1998. 


\section{APPENDIX A}

\section{Publications ANd AwARds}

\section{A.1 Publications and presentations}

1. H-W. Fang, S. M. Hsu, and J. V. Sengers, "Analysis OF UHMWPE Particles Induced Biological Response: An Approach by Microfabrication of Particles and Biophysical Modeling of Phagocytosis" World Congress for Chinese Biomedical Engineers, held Dec. 2002, Taipei, Taiwan.

2. H-W. Fang, S. M. Hsu, and J. V. Sengers, "Generation of Narrowly Distributed UHMWPE Wear Particles with Micro-fabricated Silicon Surface Textures" Biomedical Engineering: Recent Developments, Medical and Engineering Publishers, Inc, pp. 97-98, 2002.

3. S-Y. Yang, H-W. Fang, K. Siddiqi, S. M. Hsu, and P. H. Wooley, "Size and Concentration of Wear Debris UHMWPE Particles Influence Local Inflammation and Bone Resorption" Transactions of $29^{\text {th }}$ Society for Biomaterials Annual Meeting, p.611, 2003.

4. H-W. Fang, S. M. Hsu, and J. V. Sengers, "Generation of Narrowly Distributed Ultra-high Molecular Weight Polyethylene Particles by Surface Texturing Techniques" (Accepted by JBMR, Applied Biomaterials)

5. W. Ren, S. Yang, H-W. Fang. S. M. Hsu, and P. H. Wooley, "Receptor Activator of NF-KB (RANK) and RANK LIGAND (RANKL) Expression 
in Response to Varying Morphology of UHMWPE Particles in a Murine Model of Inflammation" (Accepted by Biomaterials)

6. H-W. Fang, S. M. Hsu, and J. V. Sengers, "Micro-cutting Mechanism of Surface Texture Controlled UHMWPE Wear Particle Generation", Transactions of $28^{\text {th }}$ Society for Biomaterials Annual Meeting, p.308, 2002.

7. H-W. Fang, J. V. Sengers, and S. M. Hsu, "Controlled Size and Shape UHMWPE Wear Particle Generation using a Silicon Micro-fabricated Surface Texturing Technique", Transactions of $27^{\text {th }}$ Society for Biomaterials Annual Meeting, p.30, 2001.

8. H-W. Fang, J. V. Sengers, and S. M. Hsu "Generation of Controlled UHMWPE Wear Particle Size and Shape by Surface Texturing: Relationship between Surface Feature Dimensions to Particle Size and Shape", Transactions of $27^{\text {th }}$ Society for Biomaterials Annual Meeting, p.585, 2001.

9. H-W. Fang, H-C. Liu, and T-H. Young, "Shape Discrimination of UHMWPE Particles by Fourier Descriptors and Classical Shape Parameters", Transactions of $6^{\text {th }}$ World Biomaterials Congress, p. 478, 2000.

10. H-C. Liu, R-M. Chen, F-H. Lin, and H-W. Fang, "Sintered $\beta$-dicalcium phosphate particles induce intracellular reactive oxygen species in rat osteoblasts", Biomedical Engineering Applications, Basis Communications, v11, n5, pp. 259-264, 1999 
11. H-W. Fang, M. C. Shen, U. Cho, J. Tesk, A. Christou, and S. M. Hsu, "Generation of Different UHMWPE Particle Shape by Wear through Surface Texturing", Transactions of $25^{\text {th }}$ Society for Biomaterials Annual Meeting, 506, 1999.

12. H-W. Fang, K.E. Herold, H.M. Holland, and E.G. Beach, "A Novel Stirling Engine with an Elliptic Drive", IECE Conference in Washington, $96259,1996$.

13. H-W. Fang, " Stirling Engine Performance Modeling”, M.S. Thesis, University of Maryland at College Park, 1996.

14. W. M. Lu, C. M. Lan and H-W. Fang, "Local Heat Transfer in a Stirred Vessel with and without Aeration", Journal of Chemical Engineering, Japan, v28, n6, pp. 666-672, 1995. 


\section{A.2 Awards}

1. Phi Delta Gamma Graduate Fellowship for Interdisciplinary Research, University of Maryland, April 2002.

2. $1^{\text {st }}$ Placement Awards (twice): Graduate Research Interaction Day- a presentation competition, University of Maryland at College Park, March 2001, and April 2002.

3. Student Professional Development Award: $27^{\text {th }}$ Society for Biomaterials, April 2001.

4. Academic Grant Winner: SmallTalk 2001 Conference, a microarray, microfluidics, bioMEMS conference held by Association for Laboratory Automation, San Diego, CA, Sep. 2001. 


\section{APPENDIX B}

\section{MicRofABRICATION OF STEEL SURFACE BY ELECTROCHEMICAL ETCHING}

\section{B.1 Patterning of stainless steel surface}

Two dimensional pattern were designed by a AutoCAD software. The dark features on the pattern represent the protected area from the etching process. The patterns were then transferred to a chromium coated quartz photomask by the directwriting process at the photomask manufacturer.

The polished stainless surface $(\mathrm{Ra}<0.025 \mu \mathrm{m})$ was heated on a hot plate at $205^{\circ} \mathrm{C}$ for 1 minute. This is the dehydration process to remove the water remaining on the material surface. The surface was then spin-coated 1,1,1,3,3-Hexamethyldislazane (HMDS) at $5000 \mathrm{rpm}$ for 30 seconds. The purpose of this coating is to enhance the adhesion between the wafer surface and the later coated photoresist. Shipley 1813 photoresist was spin-coated on the silicon dioxide surface at $5000 \mathrm{rpm}$ for 30 seconds. It was then baked on a hot plate at $100^{\circ} \mathrm{C}$ for 2 minutes to enhance the stability of the coated photoresist.

The dark-featured photomask was then placed in contact on the photoresist surface at a mask aligner for the following photolithography process. It was exposed to a UV source to decompose the surrounding polymer surface leaving the positive rectangular patterns which were covered by the dark features on the photomask. A post-bake process was carried out at $100^{\circ} \mathrm{C}$ for 90 seconds to accelerate and 
accomplish the photoresist decomposition. In order to fully remove the decomposed photoresist, the wafer surface was rinsed by Shipley photoresist develop 351 for 30 seconds at room temperature followed by the water rinse and blow dry processes. Up to this stage, the patterns were transferred on the photoresist layer on the stainless steel surface. The resulting photoresist pattern was then acting as the protecting layer for the following electrochemical etching of steel surface.

\section{B.2 Electrochemical etching of steel}

The stainless steel plate with patterned surface layer of photoresist was mounted on the anode end of a electrochemical cell setup as shown in Figure B.1. Ther other end is a plate of 316 stainless steel. Solution of $5 \mathrm{M} \mathrm{NaCl}$ was prepared as the electrolyte in the tank. A DC power was connected to the electrochemical cell. It is optimized with 1.125 inch between cathode and anode plates, 5V applied voltage and 1.8A electrical current, a smooth etched surface can be obtained.

\section{B.3 Etched steel surfaces}

Examples of the etched 316 stainless steel surface features are shown in Figure B.2. The corner of a square feature was rounded in Figure B.2(a) and it is found material defect on the etched surface in Figure B.2(b). There exists the limit of the resolution of the surface features because of the non-uniformity of materials and the defects of the materials. 
Stainless steel with patterned surface layer of photoresist

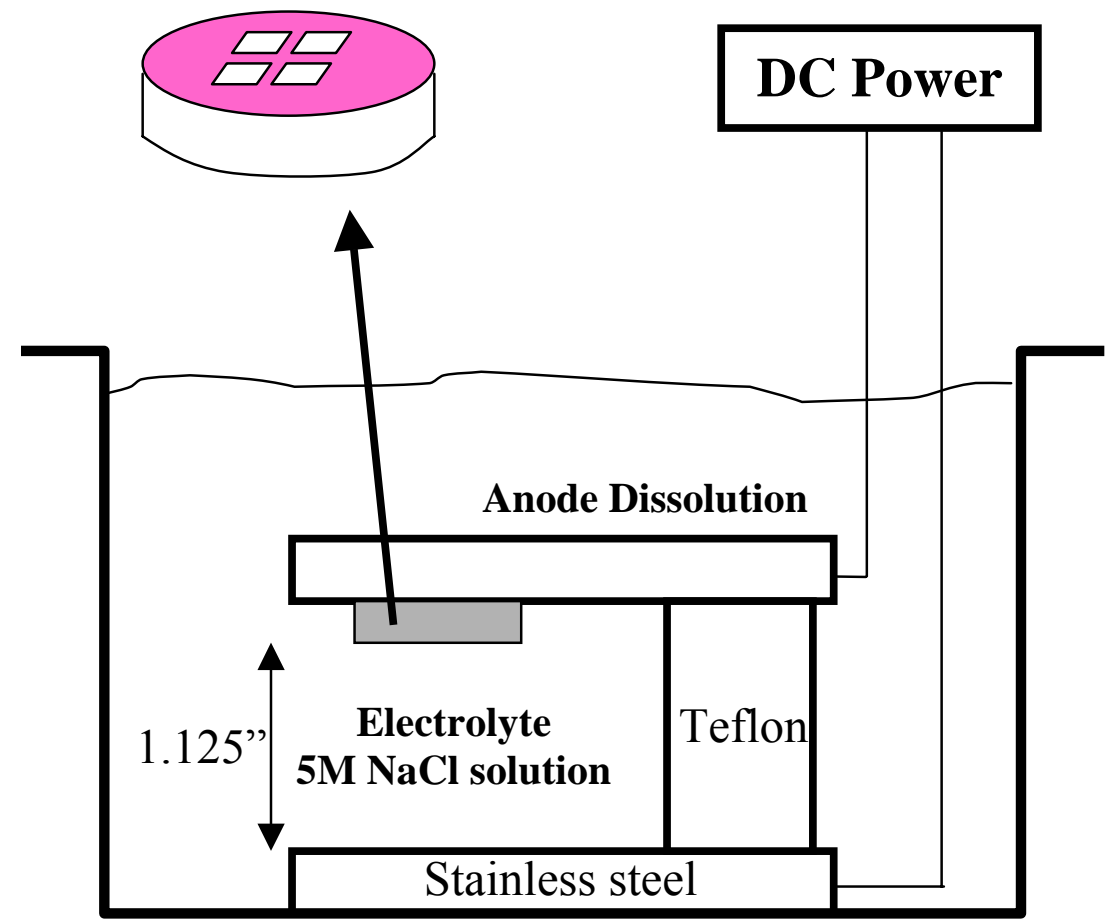

Figure B.1 A schematic of facility setup for microfabrication of stainless steel surface by electrochemical etching. 

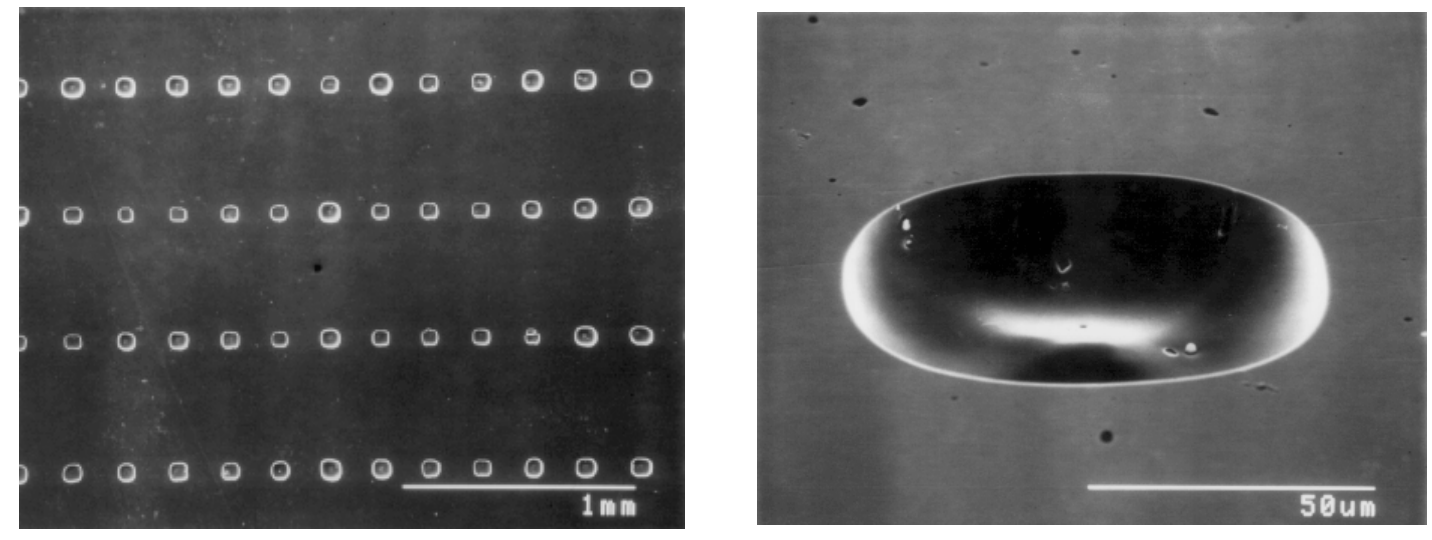

(a)
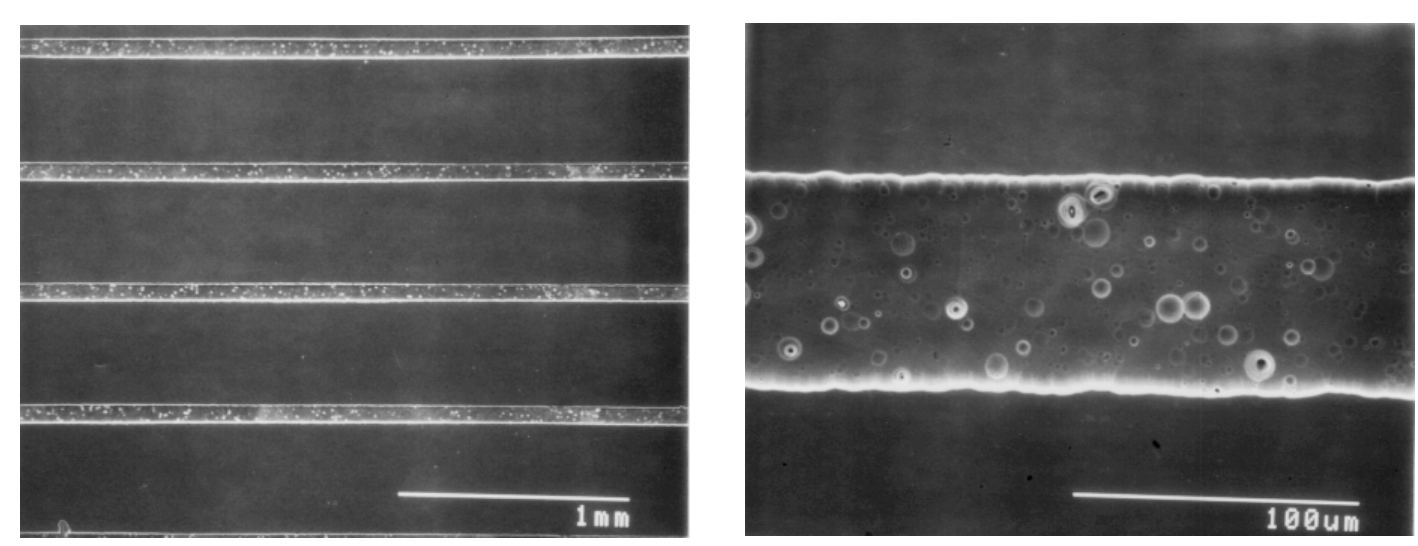

(b)

Figure B.2 Electrochemical etched stainless steel surface features (a) negative square features, (b) grove features. 


\section{APPENDIX C}

\section{Surface TEXTURE AND WEAR DATA}

\section{C.1 Dimensions of the surface texture}

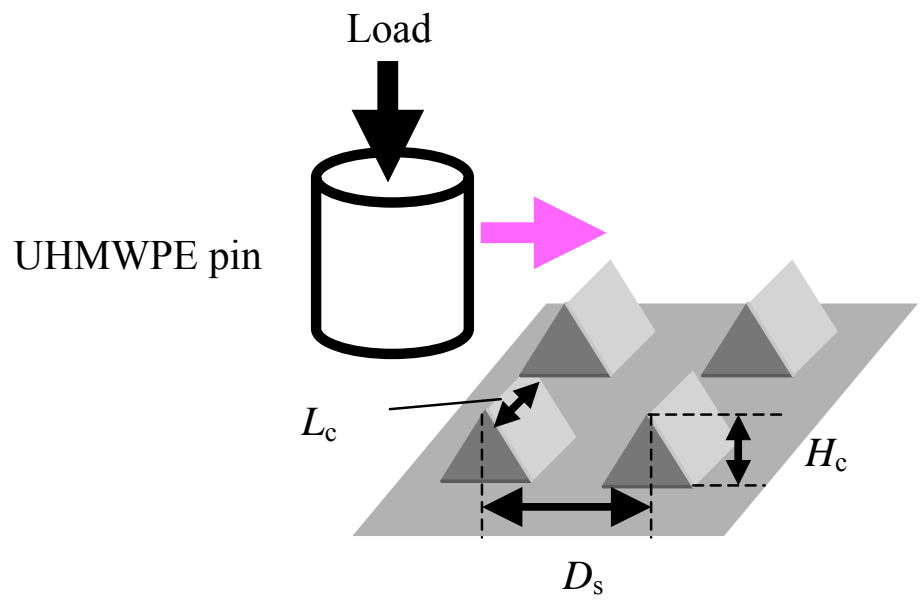

Top view of the surface texture

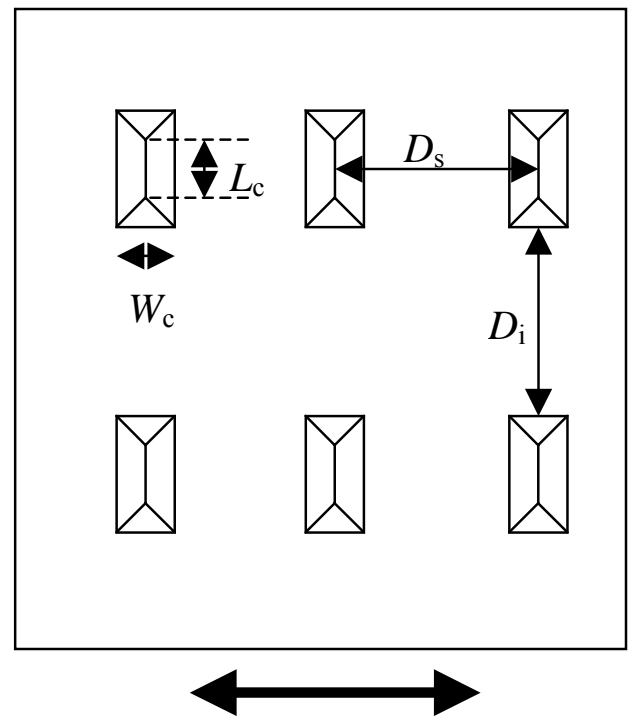

Sliding direction 


\section{C.2 Results of linear reciprocating wear tests}

The following table lists the wear testing results of UHMWPE pin sliding against various surface textures. The stroke length is $19 \mathrm{~mm}$.

\begin{tabular}{|c|c|c|c|c|c|c|c|c|c|}
\hline $\begin{array}{c}L_{\mathrm{c}} \\
(\mu \mathrm{m})\end{array}$ & $\begin{array}{c}W_{\mathrm{c}} \\
(\mu \mathrm{m})\end{array}$ & $\begin{array}{c}H_{\mathrm{c}} \\
(\mu \mathrm{m})\end{array}$ & $\begin{array}{c}D_{\mathrm{s}} \\
(\mu \mathrm{m})\end{array}$ & $\begin{array}{c}D_{\mathrm{i}} \\
(\mu \mathrm{m})\end{array}$ & $\begin{array}{c}\text { Load } \\
(\mathrm{N})\end{array}$ & $\begin{array}{c}\text { Frequency } \\
(\mathrm{Hz})\end{array}$ & $\begin{array}{c}\text { Duration } \\
(\mathrm{hr})\end{array}$ & $\begin{array}{c}\text { Wear } \\
(\mathrm{mg})\end{array}$ & $\begin{array}{c}\text { Effective } \\
\text { cutting }\end{array}$ \\
\hline 55 & 5.0 & 4.2 & 200 & 90 & 93 & 1.5 & 2 & 4.21 & yes \\
\hline 55 & 5.0 & 4.2 & 200 & 90 & 93 & 1.5 & 6 & 7.89 & yes \\
\hline 55 & 5.0 & 4.2 & 200 & 90 & 93 & 1.0 & 4 & 1.86 & yes \\
\hline 55 & 5.0 & 4.2 & 200 & 90 & 93 & 0.75 & 4 & 1.9 & yes \\
\hline 55 & 5.0 & 4.2 & 200 & 90 & 23 & 1.5 & 12 & 10.55 & yes \\
\hline 55 & 5.0 & 4.2 & 200 & 90 & 93 & 0.75 & 46 & 41.85 & yes \\
\hline 52.3 & 5.0 & 3.3 & 200 & 90 & 93 & 1.5 & 2 & 3.84 & yes \\
\hline 15.6 & 5.0 & 2.3 & 200 & 130 & 46 & 1.5 & 12 & 0.37 & yes \\
\hline 15.6 & 5.0 & 3.0 & 200 & 130 & 93 & 1.5 & 12 & 0.04 & yes \\
\hline 15.6 & 5.0 & 3.0 & 200 & 130 & 93 & 1.5 & 24 & 0.28 & yes \\
\hline 8.2 & 5.0 & 2.4 & 10 & 10 & 93 & 1.5 & 2 & 0.03 & no \\
\hline 8.2 & 5.0 & 2.4 & 10 & 10 & 93 & 1.5 & 8 & 0.04 & no \\
\hline 8.2 & 5.0 & 2.4 & 10 & 10 & 186 & 1.5 & 24 & 0.35 & no \\
\hline 8.0 & 5.0 & 2.5 & 200 & 140 & 93 & 1.5 & 2 & 0.03 & yes \\
\hline 8.0 & 5.0 & 2.5 & 200 & 140 & 11 & 1.5 & 24 & 0.14 & yes \\
\hline 8.0 & 5.0 & 2.5 & 200 & 140 & 186 & 0.75 & 124 & 5.08 & yes \\
\hline 8.0 & 5.0 & 2.5 & 200 & 140 & 186 & 0.75 & 167 & 2.79 & yes \\
\hline 7.4 & 2.0 & 0.9 & 10 & 8 & 93 & 1.5 & 12 & 0.45 & no \\
\hline 7.4 & 2.0 & 0.9 & 10 & 8 & 93 & 0.75 & 20 & 0.11 & no \\
\hline 7.4 & 2.0 & 0.9 & 10 & 8 & 186 & 1.5 & 12 & 1.63 & no \\
\hline 7.4 & 2.0 & 1.2 & 10 & 8 & 186 & 1.5 & 6 & 1.51 & no \\
\hline 5.8 & 2.0 & 1.2 & 100 & 7 & 93 & 1.5 & 48 & 0.14 & no \\
\hline 3.6 & 1.0 & 0.32 & 10 & 4 & 93 & 1.5 & 12 & 0.37 & no \\
\hline 3.6 & 1.0 & 0.32 & 10 & 4 & 186 & 1.5 & 12 & 0.42 & no \\
\hline 3.5 & 2.0 & 0.8 & 20 & 4 & 93 & 1.5 & 24 & 0.73 & yes \\
\hline 3.5 & 2.0 & 0.8 & 20 & 4 & 93 & 1.5 & 12 & 0.43 & yes \\
\hline 3.5 & 2.0 & 0.8 & 20 & 4 & 93 & 1.5 & 24 & 0.64 & yes \\
\hline 3.5 & 2.0 & 0.8 & 20 & 4 & 93 & 1.5 & 108 & 1.3 & yes \\
\hline 3.5 & 2.0 & 0.8 & 20 & 4 & 93 & 0.75 & 42 & 0.81 & yes \\
\hline 3.5 & 2.0 & 0.8 & 20 & 4 & 93 & 0.75 & 144 & 4.44 & yes \\
\hline 3.5 & 2.0 & 0.8 & 20 & 4 & 186 & 0.75 & 214 & 3.08 & yes \\
\hline 3.5 & 2.0 & 0.8 & 10 & 4 & 93 & 1.5 & 12 & 0.34 & no \\
\hline 3.5 & 2.0 & 0.8 & 10 & 4 & 93 & 0.75 & 24 & 0.41 & no \\
\hline 3.4 & 2.0 & 0.55 & 4 & 4 & 93 & 1.5 & 24 & 0.26 & no \\
\hline
\end{tabular}




\section{APPENDIX D}

\section{Modeling Of PARTiCle Generation By CORRELATION OF MODEL EXPERIMENTAL DATA}

\section{D.1 Introduction}

By the constant depth sliding experiments in section 9.4.1, we obtained the relationship between displaced volume of UHMWPE and energy input in the process was obtained. By correlating the results of the model experiments model is established to determine the shear volume (displaced volume) of UHMWPE from penetration depth calculation and the geometry of the cutting edges.

\section{D.2 Energy balance of the displaced volume of UHMWPE}

The energy input by the cutting edge to the UHMWPE material can be calculated from the force curve as shown in Figure D.1. By plotting the horizontal force as a function of the sliding distance, the work input for the displaced volume is the area under the curve toward the point A where the maximum deformation volume was reached. The displaced volume of UHMWPE is proportional to the quantity of the energy input during the sliding process and can be described as

$$
\begin{aligned}
& \int F_{\mathrm{x}} d x \propto \Delta V \\
& \Rightarrow \int F_{\mathrm{x}} d x=m \Delta V
\end{aligned}
$$

where $m$ represents a specific energy of UHMWPE deformation in the sliding process. 
Experiments were carried out with various penetration depths. The energy input and the displaced volume were calculated. Figure D.2 shows a plot of the energy input as a function of the displaced volume. The linear relationship shown in the plot verifies the relationship we proposed in equation (D.1). From a linear regression analysis of the results, the value of $m$ can be obtained from the slope of the line. Equation (D.2) can be used to estimate the material deformation in the cutting edge sliding process:

$$
\begin{aligned}
& \int F_{\mathrm{x}} d x=m \Delta V \\
& m=57.8 \frac{\mathrm{mW}}{\mathrm{mm}^{3}}
\end{aligned}
$$

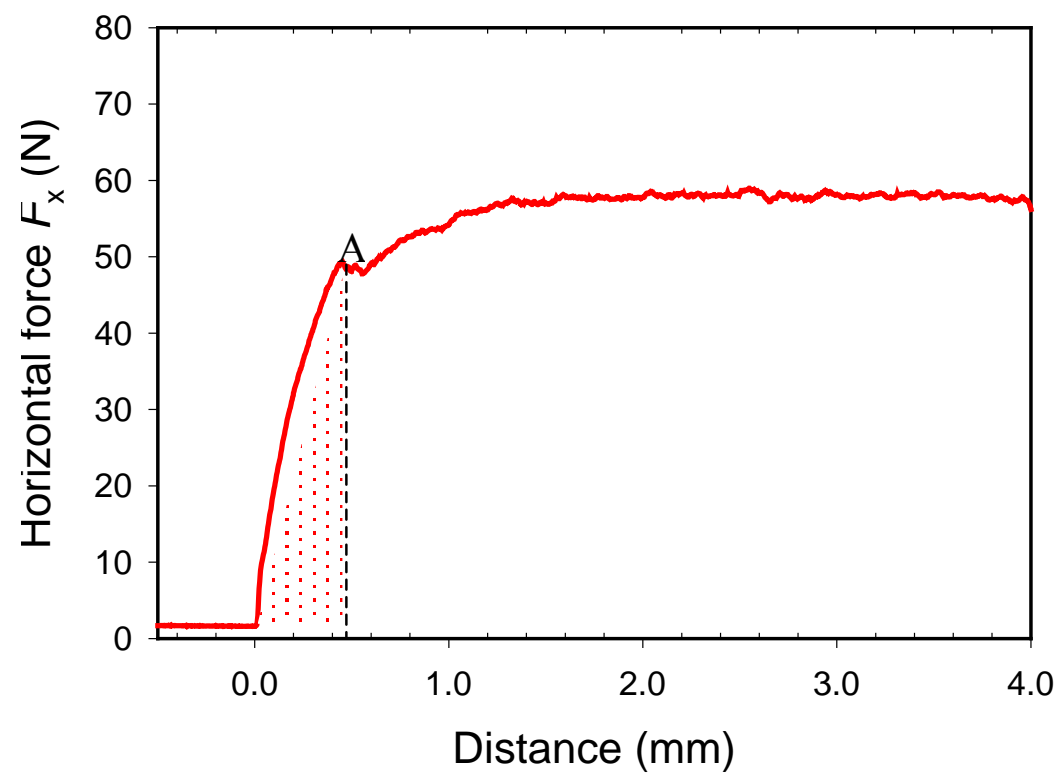

Figure D.1 Determination of the energy input from the cutting-edge sliding force curve (cutting edge length $=2 \mathrm{~mm}$, penetration depth $=400 \mu \mathrm{m}$, sliding speed $=0.5 / \mathrm{s}$ ) 


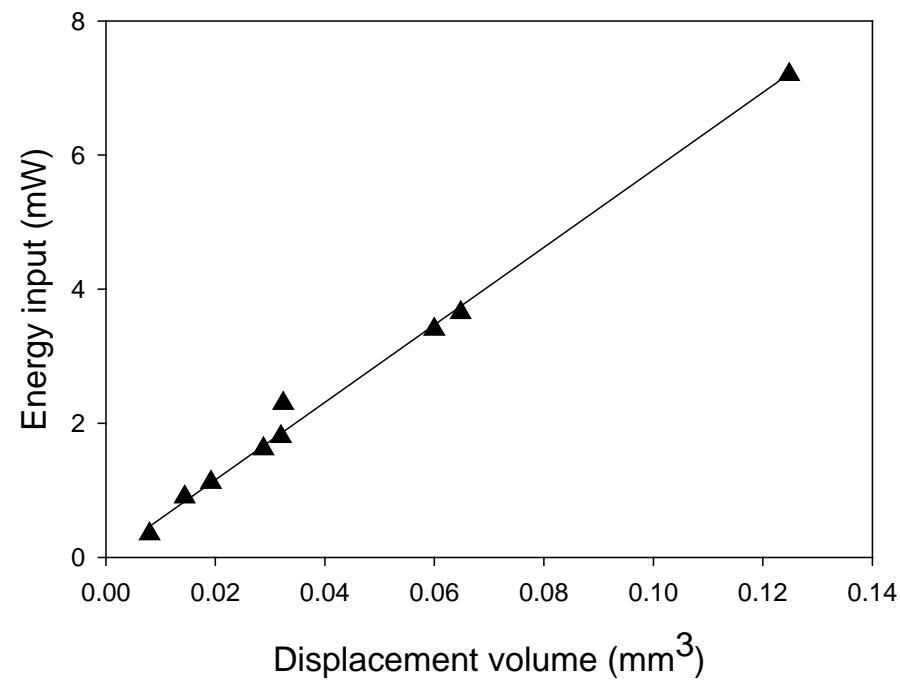

Figure D.2 Plot of energy input versus displaced volume during the cutting edge sliding process.

\section{D.3 Sliding process of the cutting edge}

In Chapter 9, we have conducted constant depth sliding experiments to investigate the sliding distance toward the cutting edge was pushed away by the strain hardening of UHMWPE. The sliding distance toward the maximum displaced volume can be used to estimate the volume of the generated particle from the dimensions of the cutting-edge length and the penetration depth. The energy input required for the volume displaced was determined and shown in equation D.2. In this section, we try to relate the resistance force and sliding distance to the penetration depth and the geometry of the cutting edge.

A larger contact area between the cutting-edge face and the UHMWPE leads to a larger material resistant force during the sliding process. The amount of matrial resistant force in the horizontal direction changes according to different cutting edge angles as shown in Figure D.3. Thus we obtain

$$
F_{\mathrm{x}} \propto L_{\mathrm{c}} \cdot H_{\mathrm{p}} \cdot \cos \theta
$$




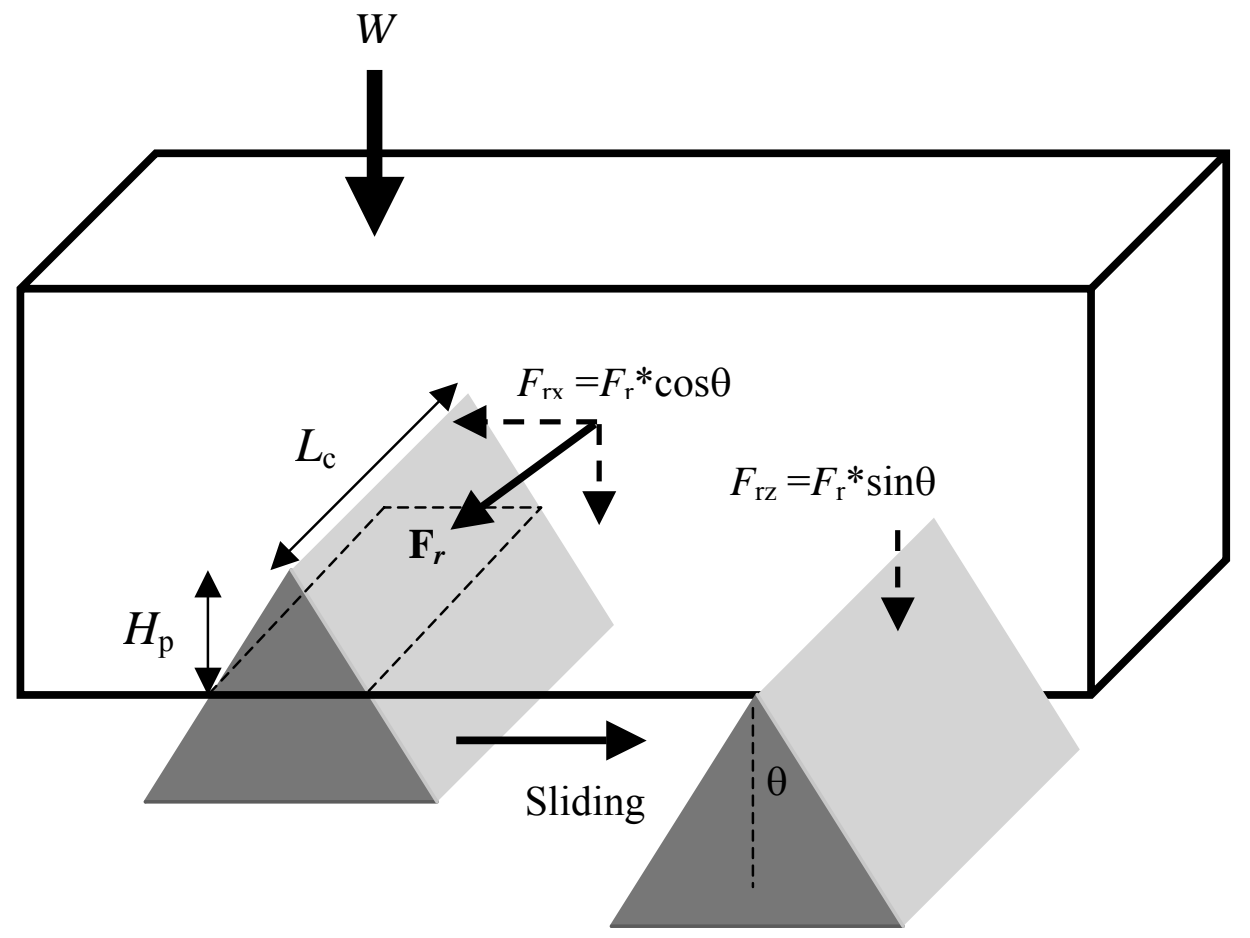

Figure D.3 A schematic representation of reacting forces in a cuttingedge sliding process.

We also have to understand the effect of cutting edge geometry on the resulting sliding distance $(\mathrm{x})$. With the constant sliding speed in the horizontal direction, the sliding distance depends on how soon the cutting edge is pushed away from UHMWPE material in the particle generation process. The cutting edge will slide longer when a larger penetration depth is introduced in the penetration process because of more time needed to lift the cutting edge to complete a cutting process. From another viewpoint, a deeper penetration depth results in a larger contact area between cutting edge and UHMWPE material. A larger volume of UHMWPE material was compressed before the cutting edge was pushed away by the strain hardening of UHMWPE. 
The cutting-edge angle affects the resolved component resistant force in $\mathrm{z}$ direction as shown in equation (D.4)

$$
F_{\mathrm{rz}}=F_{\mathrm{r}} \cdot \sin \theta
$$

Thus the acceleration of the cutting-edge push-away process is proportional to the of $F_{\mathrm{rz}}$ or $\sin \theta$. The ultimate speed when the strain hardening happens is also proportional to the $\sin \theta$. Thus the time needed to push away the cutting edge is proportional to the reciprocal of $\sin \theta$. The time needed to complete the push away process is proportional to the sliding distance. Thus we obtain the following relation:

$$
x \propto \frac{1}{\sin \theta}
$$

With the same speed, a larger penetration depth leads to a longer time to complete the micro-cutting process. This relation can be described as:

$$
x \propto H_{\mathrm{p}}
$$

From the above assumptions, we can derive the equation to calculate the energy input with known cutting edge geometry:

$$
\begin{aligned}
& F_{\mathrm{x}} \propto L_{\mathrm{c}} \cdot H_{\mathrm{p}} \cdot \cos \theta \\
& x \propto \frac{1}{\sin \theta} \cdot \mathrm{d} H_{\mathrm{p}} \\
& \Rightarrow \int F_{\mathrm{x}} \mathrm{d} x=-\int k \cdot L_{\mathrm{c}} \cdot H_{\mathrm{p}} \cdot \frac{\cos \theta}{\sin \theta} \cdot \mathrm{d} H_{\mathrm{p}} \\
& =\left.\left.k \cdot L_{\mathrm{c}} \cdot \cot \theta \cdot H_{\mathrm{p}}\right|^{2}\right|_{\mathrm{p}}, i
\end{aligned}
$$

The constant $\mathrm{k}$ is a material property parameter of UHMWPE in such a wedge-shaped cutting-edge sliding process. To determine the constant $\mathrm{k}$, we plot the energy input 
versus $\left(k \cdot L_{\mathrm{c}} \cdot \cot \theta \cdot H_{\mathrm{p}}{ }^{2}\right)$ from the results of the model experiments. From the slope of the fitted straight line as shown in Figure D.4, we can obtain the following relation:

$$
\int F_{\mathrm{x}} \mathrm{d} x=79.1 \frac{\mathrm{mW}}{\mathrm{mm}^{3}} \cdot L_{\mathrm{c}} \cdot \cot \theta \cdot H_{\mathrm{p}}{ }^{2}
$$

From equation (D.2), we have obtained the energy required for the volume displaced in the sliding process. By combining equations (D.2) and (D.8) we can estimate the particle volume based on the given cutting edge geometry and the penetration depth estimated from equation (10.8) and obtain the following equation:

$$
V_{\mathrm{p}}=1.37 \cdot L_{\mathrm{c}} \cdot \cot \theta \cdot H_{\mathrm{p}}{ }^{2}
$$

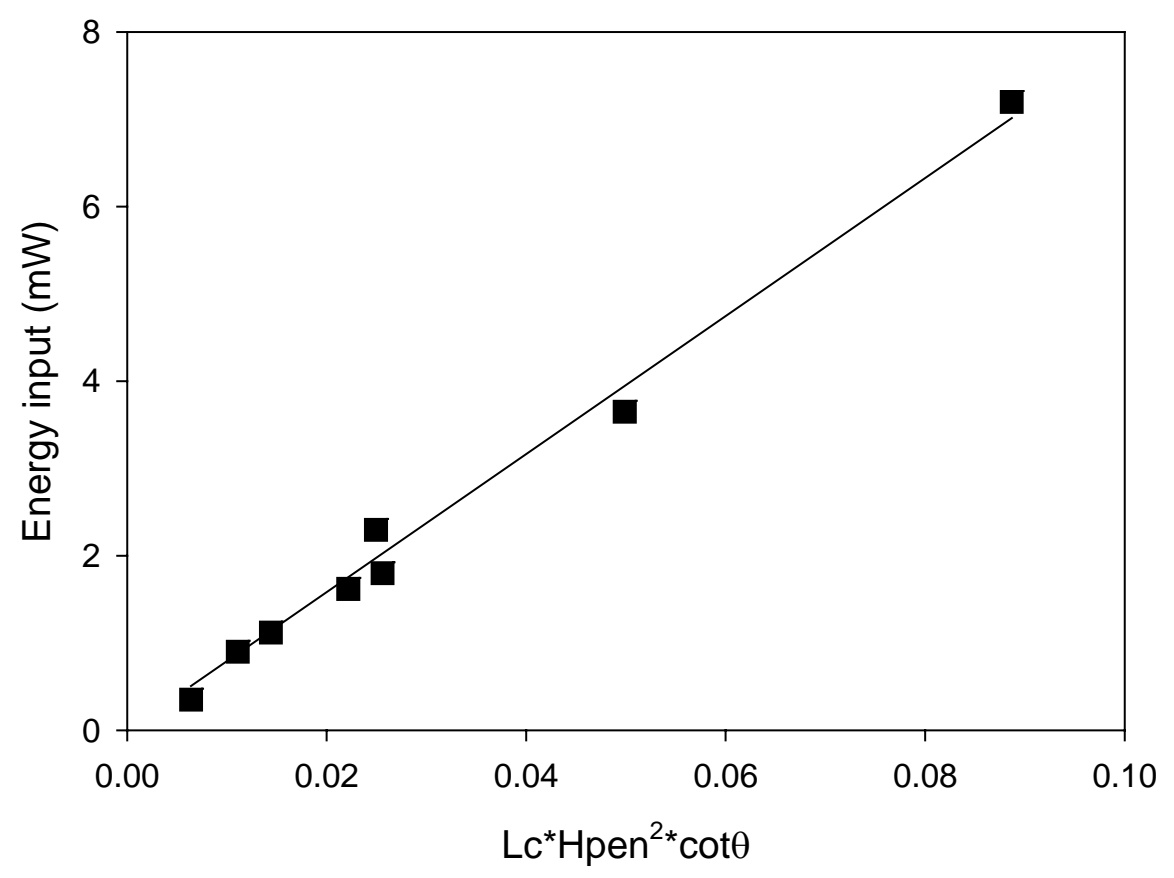

Figure D.4 Plot of energy input versus $\left(k \cdot L_{\mathrm{c}} \cdot \cot \theta \cdot H_{\mathrm{p}}{ }^{2}\right)$. 


\section{D.4 Comparison with experimental results}

Surface textures with different dimensions (cutting-edge length, angle and height) were used to generate particles. The particle volume was calculated from the SEM observations. Table D.1 lists some of the experimental results for comparison purpose.

In Figure D.5, the experimental results are compared with the model predictions by equation (D.9). In the small-particle volume region, the two sets of results are consistent. It is found, that the measured volume of the particle is smaller than the predicted volume for the larger particle. One possible reason is that the particle length is about $10 \%-15 \%$ smaller than the cutting-edge length. In the model, we have assumed that the particle length is equal to the cutting-edge length. The viscoelasticity, leading to the shrinkage of the particle dimension after the particle is released from the bulk UHMWPE, can explain the difference between predicted and the experimental results.

Table D.1 Examples of surface-texture dimensions and generated UHMWPE particle dimensions.

\begin{tabular}{|l|l|l|l|l|l|}
\hline $\begin{array}{l}\text { Cutting edge } \\
\text { length }(\mu \mathrm{m})\end{array}$ & $\begin{array}{l}\text { Cutting edge } \\
\text { height }(\mu \mathrm{m})\end{array}$ & $\begin{array}{l}\text { Cutting edge } \\
\text { angle } 2 \theta\end{array}$ & $\begin{array}{l}\text { Particle length } \\
(\mu \mathrm{m})\end{array}$ & $\begin{array}{l}\text { Particle width } \\
(\mu \mathrm{m})\end{array}$ & $\begin{array}{l}\text { Particle } \\
\text { thickness }(\mu \mathrm{m})\end{array}$ \\
\hline 55 & 4.2 & $61.5^{\circ}$ & 43.1 & 10.0 & 3.8 \\
\hline 52.3 & 3.3 & $58.5^{\circ}$ & 40.8 & 9.0 & 3.2 \\
\hline 15.6 & 2.3 & $92.4^{\circ}$ & 11.5 & 3.8 & 2.2 \\
\hline 15.2 & 3.0 & $78.5^{\circ}$ & 11.0 & 5.0 & 2.9 \\
\hline 8.0 & 2.5 & $90.0^{\circ}$ & 8.0 & 4.8 & 1.7 \\
\hline 3.5 & 0.8 & $102.6^{\circ}$ & 3.2 & 2.3 & 0.3 \\
\hline
\end{tabular}




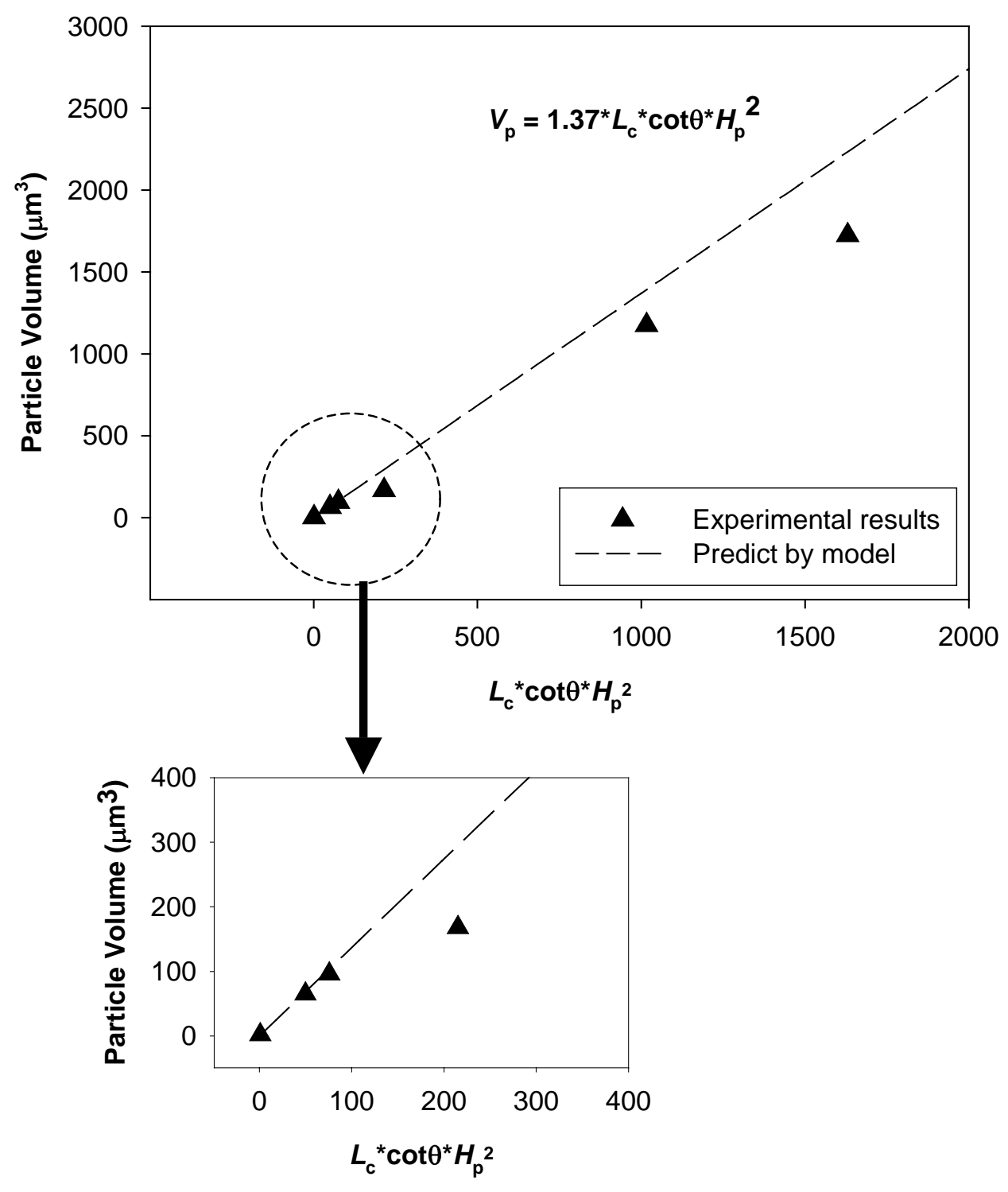

Figure D.5 Comparison of the experimental results with the prediction from a contact mechanics model. 


\section{APPENDIX E}

\section{MATLAB PROGRAM FOR Contact Mechanics Model of PARTicle GENERATION}

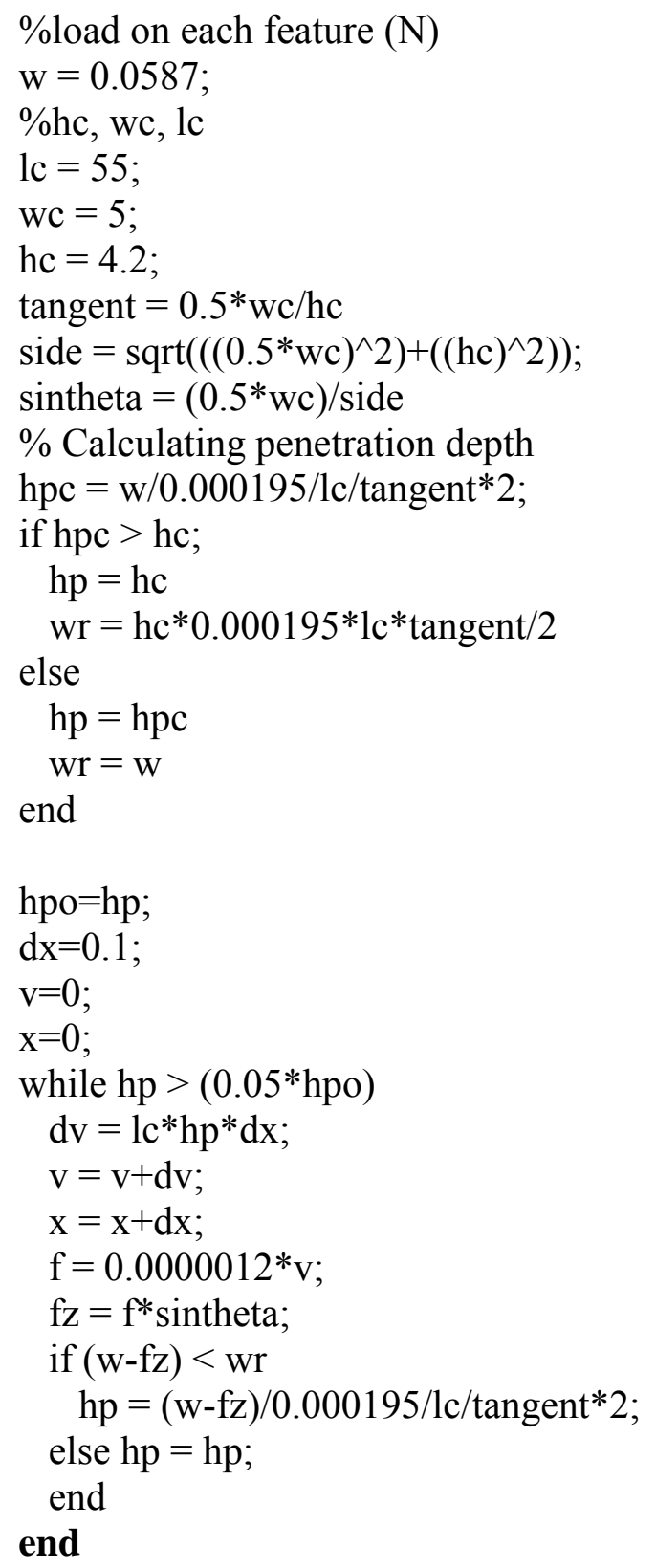


